Cochrane Database of Systematic Reviews

\title{
Laparoscopic versus open surgery for suspected appendicitis
} (Review)

Jaschinski T, Mosch CG, Eikermann M, Neugebauer EAM, Sauerland S

Jaschinski T, Mosch CG, Eikermann M, Neugebauer EAM, Sauerland S.

Laparoscopic versus open surgery for suspected appendicitis.

Cochrane Database of Systematic Reviews 2018, Issue 11. Art. No.: CD001546.

DOI: 10.1002/14651858.CD001546.pub4.

www.cochranelibrary.com 
TABLE OF CONTENTS

HEADER

ABSTRACT

PLAIN LANGUAGE SUMMARY

SUMMARY OF FINDINGS

BACKGROUND

OBJECTIVES

METHODS

RESULTS

Figure 1.

Figure 2.

Figure 3.

DISCUSSION

AUTHORS' CONCLUSIONS

ACKNOWLEDGEMENTS

REFERENCES

CHARACTERISTICS OF STUDIES

DATA AND ANALYSES

Analysis 1.1. Comparison 1 Laparoscopic versus open appendectomy in adults or adolescents, Outcome 1 Pain intensity on day 1.

Analysis 1.2. Comparison 1 Laparoscopic versus open appendectomy in adults or adolescents, Outcome 2 Wound infections.

Analysis 1.3. Comparison 1 Laparoscopic versus open appendectomy in adults or adolescents, Outcome 3 Intra-abdominal abscesses.

Analysis 1.4. Comparison 1 Laparoscopic versus open appendectomy in adults or adolescents, Outcome 4 Length of hospital stay.

Analysis 1.5. Comparison 1 Laparoscopic versus open appendectomy in adults or adolescents, Outcome 5 Time until return to normal activity.

Analysis 2.1. Comparison 2 Laparoscopic versus open appendectomy in children, Outcome 1 Pain intensity on day 1 (cm VAS).

Analysis 2.2. Comparison 2 Laparoscopic versus open appendectomy in children, Outcome 2 Wound infections.

Analysis 2.3. Comparison 2 Laparoscopic versus open appendectomy in children, Outcome 3 Intra-abdominal abscesses. .....

Analysis 2.4. Comparison 2 Laparoscopic versus open appendectomy in children, Outcome 4 Length of hospital stay.

Analysis 2.5. Comparison 2 Laparoscopic versus open appendectomy in children, Outcome 5 Time until return to normal activity.

Analysis 3.1. Comparison 3 Subgroup analysis (publication date) - laparoscopic versus open appendectomy in adults or adolescents, Outcome 1 Pain intensity on day 1.

Analysis 3.2. Comparison 3 Subgroup analysis (publication date) - laparoscopic versus open appendectomy in adults or adolescents, Outcome 2 Wound infections.

Analysis 3.3. Comparison 3 Subgroup analysis (publication date) - laparoscopic versus open appendectomy in adults or adolescents, Outcome 3 Intra-abdominal abscesses.

Analysis 3.4. Comparison 3 Subgroup analysis (publication date) - laparoscopic versus open appendectomy in adults or adolescents, Outcome 4 Length of hospital stay.

Analysis 3.5. Comparison 3 Subgroup analysis (publication date) - laparoscopic versus open appendectomy in adults or adolescents, Outcome 5 Time until return to normal activity.

Analysis 4.1. Comparison 4 Subgroup analysis (publication date) - Laparoscopic versus open appendectomy in children, Outcome 1 Wound infections.

Analysis 4.2. Comparison 4 Subgroup analysis (publication date) - Laparoscopic versus open appendectomy in children, Outcome 2 Intra-abdominal abscesses.

Analysis 4.3. Comparison 4 Subgroup analysis (publication date) - Laparoscopic versus open appendectomy in children, Outcome 3 Time until return to normal activity.

Analysis 4.4. Comparison 4 Subgroup analysis (publication date) - Laparoscopic versus open appendectomy in children, Outcome 4 Length of hospital stay. 
[Intervention Review]

\section{Laparoscopic versus open surgery for suspected appendicitis}

Thomas Jaschinski ${ }^{1}$, Christoph G Mosch ${ }^{1}$, Michaela Eikermann², Edmund AM Neugebauer ${ }^{3}$, Stefan Sauerland ${ }^{4}$

1 Institute for Research in Operative Medicine (IFOM) - Department for Evidence-based Health Services Research, University Witten/ Herdecke, Cologne, Germany. 2Department of Evidence-based medicine, Medical advisory service of social health insurance (MDS), Essen, Germany. ${ }^{3}$ Brandenburg Medical School Theodor Fontane 3, Neuruppin, Germany. ${ }^{4}$ Department of Non-Drug Interventions, Institute for Quality and Efficiency in Health Care (IQWiG), Cologne, Germany

Contact address: Thomas Jaschinski, Institute for Research in Operative Medicine (IFOM) - Department for Evidence-based Health Services Research, University Witten/Herdecke, Ostmerheimer Str. 200 (Building 38), Cologne, 51109, Germany. thomas.jaschinski@uniwh.de, tjaschinski@web.de.

Editorial group: Cochrane Colorectal Cancer Group.

Publication status and date: New search for studies and content updated (no change to conclusions), published in Issue 11, 2018.

Citation: Jaschinski T, Mosch CG, Eikermann M, Neugebauer EAM, Sauerland S. Laparoscopic versus open surgery for suspected appendicitis. Cochrane Database of Systematic Reviews 2018, Issue 11. Art. No.: CD001546. DOI: 10.1002/14651858.CD001546.pub4.

Copyright ( 2018 The Cochrane Collaboration. Published by John Wiley \& Sons, Ltd.

\section{A B S T R A C T}

\section{Background}

The removal of the acute appendix is one of the most frequently performed surgical procedures. Open surgery associated with therapeutic efficacy has been the treatment of choice for acute appendicitis. However, in consequence of the evolution of endoscopic surgery, the operation can also be performed with minimally invasive surgery. Due to smaller incisions, the laparoscopic approach may be associated with reduced postoperative pain, reduced wound infection rate, and shorter time until return to normal activity.

This is an update of the review published in 2010.

\section{Objectives}

To compare the effects of laparoscopic appendectomy (LA) and open appendectomy (OA) with regard to benefits and harms.

\section{Search methods}

We searched the Cochrane Central Register of Controlled Trials (CENTRAL), Ovid MEDLINE and Embase (9 February 2018). We identified proposed and ongoing studies from World Health Organization (WHO) International Clinical Trials Registry Platform (ICTRP), ClinicalTrials.gov and EU Clinical Trials Register (9 February 2018). We handsearched reference lists of identified studies and the congress proceedings of endoscopic surgical societies.

\section{Selection criteria}

We included randomised controlled trials (RCTs) comparing LA versus OA in adults or children.

\section{Data collection and analysis}

Two review authors independently selected studies, assessed the risk of bias, and extracted data. We performed the meta-analyses using Review Manager 5. We calculated the Peto odds ratio (OR) for very rare outcomes, and the mean difference (MD) for continuous outcomes (or standardised mean differences (SMD) if researchers used different scales such as quality of life) with $95 \%$ confidence intervals (CI). We used GRADE to rate the quality of the evidence.

\section{Main results}

We identified 85 studies involving 9765 participants. Seventy-five trials included 8520 adults and 10 trials included 1245 children. Most studies had risk of bias issues, with attrition bias being the largest source across studies due to incomplete outcome data. 
In adults, pain intensity on day one was reduced by $0.75 \mathrm{~cm}$ on a $10 \mathrm{~cm}$ VAS after LA (MD $-0.75,95 \% \mathrm{Cl}-1.04$ to $-0.45 ; 20 \mathrm{RCTs} ; 2421$ participants; low-quality evidence). Wound infections were less likely after LA (Peto OR $0.42,95 \% \mathrm{Cl} 0.35$ to $0.51 ; 63$ RCTs; 7612 participants; moderate-quality evidence), but the incidence of intra-abdominal abscesses was increased following LA (Peto OR 1.65, 95\% Cl 1.12 to 2.43; 53 RCTs; 6677 participants; moderate-quality evidence).

The length of hospital stay was shortened by one day after LA (MD -0.96, 95\% Cl -1.23 to -0.70; 46 RCTs; 5127 participant; low-quality evidence). The time until return to normal activity occurred five days earlier after LA than after OA (MD $-4.97,95 \% \mathrm{Cl}-6.77$ to $-3.16 ; 17$ RCTs; 1653 participants; low-quality evidence). Two studies showed better quality of life scores following LA, but used different scales, and therefore no pooled estimates were presented. One used the SF-36 questionnaire two weeks after surgery and the other used the Gastrointestinal Quality of Life Index six weeks and six months after surgery (both low-quality evidence).

In children, we found no differences in pain intensity on day one (MD-0.80,95\% Cl-1.65 to $0.05 ; 1 \mathrm{RCT} ; 61$ participants; low-quality evidence), intra-abdominal abscesses after LA (Peto OR $0.54,95 \% \mathrm{Cl} 0.24$ to 1.22; 9 RCTs; 1185 participants; low-quality evidence) or time until return to normal activity (MD $-0.50,95 \% \mathrm{Cl}-1.30$ to $0.30 ; 1 \mathrm{RCT} ; 383$ participants; moderate-quality evidence). However, wound infections were less likely after LA (Peto OR $0.25,95 \% \mathrm{Cl} 0.15$ to $0.42 ; 10 \mathrm{RCTs} ; 1245$ participants; moderate-quality evidence) and the length of hospital stay was shortened by 0.8 days after LA (MD $-0.81,95 \% \mathrm{Cl}-1.01$ to $-0.62 ; 6 \mathrm{RCTs} ; 316$ participants; low-quality evidence). Quality of life was not reported in any of the included studies.

\section{Authors' conclusions}

Except for a higher rate of intra-abdominal abscesses after LA in adults, LA showed advantages over OA in pain intensity on day one, wound infections, length of hospital stay and time until return to normal activity in adults. In contrast, LA showed advantages over OA in wound infections and length of hospital stay in children. Two studies reported better quality of life scores in adults. No study reported this outcome in children. However, the quality of evidence ranged from very low to moderate and some of the clinical effects of LA were small and of limited clinical relevance. Future studies with low risk of bias should investigate, in particular, the quality of life in children.

\section{PLAIN LANGUAGE SUMMARY}

\section{Laparoscopic surgery compared to open surgery for suspected appendicitis}

\section{Review question}

We reviewed the evidence about the effects of the open operation technique and the minimally invasive procedure in persons with suspected appendicitis.

\section{Background}

In the right lower part of the abdomen there is a small blind ending intestinal tube, called the appendix. Inflammation of the appendix is called appendicitis which is most frequent in children and young adults. Most cases require emergency surgery in order to avoid rupture of the appendix into the abdomen. During the operation, called appendectomy, the inflamed appendix is surgically removed. The traditional surgical approach involves a small incision (about $5 \mathrm{~cm}$ or 2 inches) in the right lower abdominal wall. Alternatively, it is possible to remove the inflamed appendix using another surgical technique, known as laparoscopic appendectomy. This operation requires three very small incisions (each about $1 \mathrm{~cm}$ or $1 / 2$ inch). Then the surgeon introduces a camera and instruments into the abdomen and removes the appendix.

\section{Study characteristics}

We included 85 studies involving 9765 participants, of which 75 trials compared laparoscopic appendectomy versus open appendectomy in adults. The remaining 10 studies included only children. The evidence is current to February 2018.

\section{Key results}

The main advantages of laparoscopic compared to open appendectomy were reduced postsurgical pain, reduced risk of wound infection, shorter hospital stay, and more rapid return to normal activities in adults. In contrast, laparoscopic appendectomy showed advantages over open appendectomy in wound infections and shorter hospital stay in children. Two studies reported that adults who received laparoscopic appendectomy had better quality of life two weeks, six weeks, and six months after surgery. Data from children were not available. As for disadvantages of laparoscopic appendectomy, a higher rate of intra-abdominal abscesses were identified in adults but not in children. Except for a trend towards decreased intra-abdominal abscesses after LA, the results for children were similar to those seen in adults.

\section{Quality of the evidence}

The quality of the evidence varied from moderate to low because of poorly conducted studies. 
SUMMARY OF FINDINGS

\section{Summary of findings for the main comparison. Laparoscopic versus open appendectomy in adults or adolescents for suspected appendicitis}

Laparoscopic versus open appendectomy in adults or adolescents for suspected appendicitis

Patient or population: patients with suspected appendicitis

Settings: most trials were single institutions across Europe, Asia, North America, South America, Australia and South Africa (frequency in descending order) Intervention: laparoscopic versus open appendectomy in adults or adolescents

\begin{tabular}{|c|c|c|c|c|c|c|}
\hline \multirow[t]{2}{*}{ Outcomes } & \multicolumn{2}{|c|}{ Illustrative comparative risks* $(95 \% \mathrm{CI})$} & \multirow{2}{*}{$\begin{array}{l}\text { Relative effect } \\
(95 \% \mathrm{CI})\end{array}$} & \multirow{2}{*}{$\begin{array}{l}\text { No of Partici- } \\
\text { pants } \\
\text { (studies) }\end{array}$} & \multirow{2}{*}{$\begin{array}{l}\text { Quality of the } \\
\text { evidence } \\
\text { (GRADE) }\end{array}$} & \multirow[t]{2}{*}{ Comments } \\
\hline & open appendectomy & laparoscopic appendectomy & & & & \\
\hline $\begin{array}{l}\text { Pain intensity on } \\
\text { day } 1\end{array}$ & $\begin{array}{l}\text { The mean pain intensity on } \\
\text { day } 1 \text { ranged from } 1.36 \text { to } \\
\text { 5.9. }\end{array}$ & $\begin{array}{l}\text { The mean pain intensity on day } 1 \text { in the } \\
\text { laparoscopic groups was } \\
\mathbf{0 . 7 5} \mathbf{c m} \text { lower (on a 10-cm VAS scale) } \\
\text { (1.04 to } 0.45 \text { lower) }\end{array}$ & & $\begin{array}{l}2421 \\
\text { (20 studies) }\end{array}$ & $\begin{array}{l}\oplus \oplus \odot \odot \\
\operatorname{low}^{1}\end{array}$ & \\
\hline $\begin{array}{l}\text { Wound infec- } \\
\text { tions }\end{array}$ & 86 per 1000 & $\begin{array}{l}38 \text { per } 1000 \\
(32 \text { to } 46)\end{array}$ & $\begin{array}{l}\text { Peto OR } \\
\mathbf{0 . 4 2}(0.35 \text { to } \\
0.51)\end{array}$ & $\begin{array}{l}7612 \\
\text { (63 studies) }\end{array}$ & $\begin{array}{l}\oplus \oplus \oplus \ominus \\
\text { moderate }^{2}\end{array}$ & $\begin{array}{l}\text { Two studies } \\
\text { reported no } \\
\text { events }\end{array}$ \\
\hline \multirow{2}{*}{$\begin{array}{l}\text { Intra-abdominal } \\
\text { abscesses }\end{array}$} & \multicolumn{2}{|l|}{ Study population } & \multirow{2}{*}{$\begin{array}{l}\text { Peto OR } \\
\mathbf{1 . 6 5}(1.12 \text { to } \\
2.43)\end{array}$} & \multirow{2}{*}{$\begin{array}{l}6677 \\
\text { (53 studies) }\end{array}$} & \multirow{2}{*}{$\begin{array}{l}\oplus \oplus \oplus \ominus \\
\text { moderate }^{2}\end{array}$} & \multirow{2}{*}{$\begin{array}{l}\text { Twenty-eight } \\
\text { studies report- } \\
\text { ed no events }\end{array}$} \\
\hline & 12 per 1000 & $\begin{array}{l}20 \text { per } 1000 \\
(13 \text { to } 29)\end{array}$ & & & & \\
\hline $\begin{array}{l}\text { Time until re- } \\
\text { turn to normal } \\
\text { activities (days) }\end{array}$ & $\begin{array}{l}\text { The mean time until return } \\
\text { to normal activities (days) } \\
\text { ranged from } 3.2 \text { to } 32.4 \text {. }\end{array}$ & $\begin{array}{l}\text { The mean time until return to nor- } \\
\text { mal activity (days) in the laparoscopic } \\
\text { groups was } \\
\mathbf{4 . 9 7} \text { shorter } \\
\text { (6.77 to } 3.16 \text { shorter) }\end{array}$ & & $\begin{array}{l}1653 \\
\text { (17 studies) }\end{array}$ & $\begin{array}{l}\oplus \oplus \odot \ominus \\
\text { low }^{1}\end{array}$ & \\
\hline $\begin{array}{l}\text { Quality of life } \\
\text { Scale used: }\end{array}$ & \multicolumn{4}{|c|}{$\begin{array}{l}\text { Kaplan } 2009 \text { used the Gastro-intestinal Quality of Life Index questionnaire and found significant differences in } \\
\text { favour of laparoscopic appendectomy } 6 \text { weeks and } 6 \text { months after surgery (each, } P<0.05) \text {. }\end{array}$} & \multicolumn{2}{|l|}{$\begin{array}{l}\oplus \oplus \odot \odot \\
\operatorname{low}^{3}\end{array}$} \\
\hline
\end{tabular}


tionnaire

*The basis for the assumed risk (e.g. the median control group risk across studies) is provided in footnotes. The corresponding risk (and its $95 \%$ confidence interval) is based on the assumed risk in the comparison group and the relative effect of the intervention (and its $95 \% \mathrm{Cl}$ ).

Cl: Confidence interval; Peto OR: Peto odds ratio; SF-36: Short form-36

GRADE Working Group grades of evidence

High quality: Further research is very unlikely to change our confidence in the estimate of effect.

Moderate quality: Further research is likely to have an important impact on our confidence in the estimate of effect and may change the estimate.

Low quality: Further research is very likely to have an important impact on our confidence in the estimate of effect and is likely to change the estimate.

Very low quality: We are very uncertain about the estimate.

1 Downgraded two levels for risk of bias (selection and detection bias) and inconsistency (heterogeneity)

2 Downgraded one level for risk of bias (selection and detection bias)

3 Downgraded two levels for risk of bias (selection and detection bias) and imprecision (small sample size)

\section{Summary of findings 2. Laparoscopic versus open appendectomy in children for suspected appendicitis}

\section{Laparoscopic versus open appendectomy in children for suspected appendicitis}

Patient or population: patients with suspected appendicitis

Settings: most trials were single institutions across Europe, Asia, North America, South America, Australia and South Africa (frequency in descending order)

Intervention: laparoscopic versus open appendectomy in children

\begin{tabular}{|c|c|c|c|c|c|c|}
\hline \multirow[t]{2}{*}{ Outcomes } & \multicolumn{2}{|c|}{ Illustrative comparative risks* $(95 \% \mathrm{Cl})$} & \multirow{2}{*}{$\begin{array}{l}\text { Relative effect } \\
(95 \% \mathrm{CI})\end{array}$} & \multirow{2}{*}{$\begin{array}{l}\text { No of Partici- } \\
\text { pants } \\
\text { (studies) }\end{array}$} & \multirow{2}{*}{$\begin{array}{l}\text { Quality of the } \\
\text { evidence } \\
\text { (GRADE) }\end{array}$} & \multirow[t]{2}{*}{ Comments } \\
\hline & open appendectomy & laparoscopic appendectomy & & & & \\
\hline $\begin{array}{l}\text { Pain intensity on } \\
\text { day }\end{array}$ & $\begin{array}{l}\text { The mean pain intensity } \\
\text { on day } 1 \text { was } 2.5 \text {. }\end{array}$ & $\begin{array}{l}\text { The mean pain intensity on day } 1 \text { in the la- } \\
\text { paroscopic groups was } \\
\mathbf{0 . 8} \mathbf{~ c m ~ l o w e r ~ ( o n ~ a ~ 1 0 - c m ~ V A S ~ s c a l e ) ~ ( 1 . 6 5 ~} \\
\text { lower to } 0.05 \text { higher) }\end{array}$ & & $\begin{array}{l}61 \\
\text { (1 study) }\end{array}$ & $\begin{array}{l}\oplus \oplus \odot \odot \\
\text { low }^{1}\end{array}$ & \\
\hline $\begin{array}{l}\text { Wound infec- } \\
\text { tions (rate) }\end{array}$ & 76 per 1000 & $\begin{array}{l}20 \text { per } 1000 \\
\text { (12 to } 33)\end{array}$ & $\begin{array}{l}\text { Peto OR } 0.25 \\
(0.15 \text { to } 0.42)\end{array}$ & $\begin{array}{l}1245 \\
\text { (10 studies) }\end{array}$ & $\begin{array}{l}\oplus \oplus \oplus \odot \\
\text { moderate }^{2}\end{array}$ & $\begin{array}{l}\text { Four studies } \\
\text { reported no } \\
\text { events }\end{array}$ \\
\hline
\end{tabular}




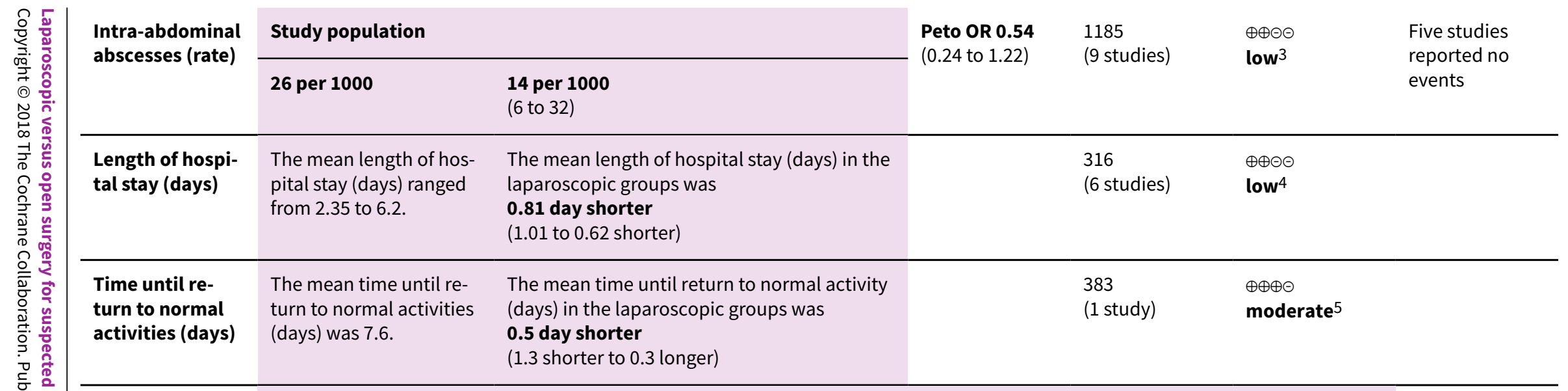

\section{Quality of life Not reported}

*The basis for the assumed risk (e.g. the median control group risk across studies) is provided in footnotes. The corresponding risk (and its $95 \%$ confidence interval) is based on the assumed risk in the comparison group and the relative effect of the intervention (and its $95 \% \mathrm{Cl}$ ).

Cl: Confidence interval; Peto OR: Peto odds ratio

GRADE Working Group grades of evidence

High quality: Further research is very unlikely to change our confidence in the estimate of effect.

Moderate quality: Further research is likely to have an important impact on our confidence in the estimate of effect and may change the estimate.

Low quality: Further research is very likely to have an important impact on our confidence in the estimate of effect and is likely to change the estimate.

Very low quality: We are very uncertain about the estimate.

1 Downgraded two levels for risk of bias (selection and detection bias) and imprecision (small sample size)

2 Downgraded one level for risk of bias (selection and detection bias)

3 Downgraded two levels for risk of bias (selection and detection bias) and imprecision (wide confidence interval)

4 Downgraded two levels for risk of bias (selection and detection bias) and inconsistency (heterogeneity)

5 Downgraded one level for imprecision (wide confidence interval)

\section{Summary of findings 3. Subgroup analysis (publication date) - Laparoscopic versus open appendectomy in adults or adolescents for suspected} appendicitis

Subgroup analysis (publication date) - Laparoscopic versus open appendectomy in adults or adolescents for suspected appendicitis

Patient or population: patients with suspected appendicitis

Settings: most trials were single institutions across Europe, Asia, North America, South America, Australia, and South Africa (frequency in descending order)

Intervention: subgroup analysis (publication date) - laparoscopic versus open appendectomy in adults or adolescents 


\begin{tabular}{|c|c|c|c|c|c|c|}
\hline \multirow[t]{2}{*}{ Outcomes } & \multicolumn{2}{|c|}{ Illustrative comparative risks ${ }^{\star}(95 \% \mathrm{Cl})$} & \multirow{2}{*}{$\begin{array}{l}\text { Relative effect } \\
(95 \% \mathrm{Cl})\end{array}$} & \multirow{2}{*}{$\begin{array}{l}\text { No of Partici- } \\
\text { pants } \\
\text { (studies) }\end{array}$} & \multirow{2}{*}{$\begin{array}{l}\text { Quality of the } \\
\text { evidence } \\
\text { (GRADE) }\end{array}$} & \multirow[t]{2}{*}{ Comments } \\
\hline & open appendectomy & laparoscopic appendectomy & & & & \\
\hline $\begin{array}{l}\text { Pain intensity on } \\
\text { day } 1\end{array}$ & $\begin{array}{l}\text { The mean pain intensity on } \\
\text { day } 1 \text { ranged from } 1.36 \text { to } \\
4.53 \text {. }\end{array}$ & $\begin{array}{l}\text { The mean pain intensity on day } 1 \text { in the la- } \\
\text { paroscopic groups was } \\
\mathbf{0 . 5 2} \mathbf{~ c m ~ l o w e r ~ ( o n ~ a ~ 1 0 - c m ~ V A S ~ s c a l e ) ~} \\
\text { (0.98 to } 0.06 \text { lower) }\end{array}$ & & $\begin{array}{l}613 \\
\text { (7 studies) }\end{array}$ & $\begin{array}{l}\oplus \oplus \odot \odot \\
\text { low }^{1}\end{array}$ & \\
\hline \multirow{2}{*}{$\begin{array}{l}\text { Wound infec- } \\
\text { tions }\end{array}$} & \multicolumn{2}{|l|}{ Study population } & \multirow{2}{*}{$\begin{array}{l}\text { Peto OR } 0.37 \\
(0.27 \text { to } 0.51)\end{array}$} & \multirow{2}{*}{$\begin{array}{l}2217 \\
\text { (19 studies) }\end{array}$} & \multirow{2}{*}{$\begin{array}{l}\oplus \oplus \oplus \ominus \\
\text { moderate }^{2}\end{array}$} & \multirow{2}{*}{$\begin{array}{l}\text { One study } \\
\text { reported no } \\
\text { events }\end{array}$} \\
\hline & 121 per 1000 & $\begin{array}{l}49 \text { per } 1000 \\
\text { (36 to } 66)\end{array}$ & & & & \\
\hline \multirow{2}{*}{$\begin{array}{l}\text { Intra-abdominal } \\
\text { abscesses }\end{array}$} & \multicolumn{2}{|l|}{ Study population } & \multirow{2}{*}{$\begin{array}{l}\text { Peto OR } 0.82 \\
(0.44 \text { to } 1.51)\end{array}$} & \multirow{2}{*}{$\begin{array}{l}1466 \\
\text { (11 studies) }\end{array}$} & \multirow{2}{*}{$\begin{array}{l}\oplus \oplus \Theta \Theta \\
\text { low }^{3}\end{array}$} & \multirow{2}{*}{$\begin{array}{l}\text { Four studies } \\
\text { reported no } \\
\text { events }\end{array}$} \\
\hline & 31 per 1000 & $\begin{array}{l}26 \text { per } 1000 \\
(14 \text { to } 46)\end{array}$ & & & & \\
\hline $\begin{array}{l}\text { Length of hospi- } \\
\text { tal stay }\end{array}$ & $\begin{array}{l}\text { The mean length of hospital } \\
\text { stay (days) ranged from } 0.88 \\
\text { to } 11.9 .\end{array}$ & $\begin{array}{l}\text { The mean length of hospital stay (days) in } \\
\text { the laparoscopic groups was } \\
\mathbf{0 . 8 4} \text { day shorter } \\
\text { (1.23 to } 0.44 \text { shorter) }\end{array}$ & & $\begin{array}{l}1977 \\
\text { (17 studies) }\end{array}$ & $\begin{array}{l}\oplus \oplus \odot \odot \\
\text { low }^{1}\end{array}$ & \\
\hline $\begin{array}{l}\text { Time until re- } \\
\text { turn to normal } \\
\text { activity }\end{array}$ & $\begin{array}{l}\text { The mean time until return } \\
\text { to normal activities (days) } \\
\text { ranged from } 3.2 \text { to } 8.7 \text {. }\end{array}$ & $\begin{array}{l}\text { The mean time until return to normal ac- } \\
\text { tivity (days) in the laparoscopic groups } \\
\text { was } \\
\mathbf{2 . 2 7} \text { days shorter } \\
\text { ( } 5.81 \text { shorter to } 1.26 \text { longer) }\end{array}$ & & $\begin{array}{l}380 \\
\text { (3 studies) }\end{array}$ & $\begin{array}{l}\oplus \odot \odot \odot \\
\text { very low } 4\end{array}$ & \\
\hline $\begin{array}{l}\text { Quality of life } \\
\text { Scale used: } \\
\text { Gastro-intestinal } \\
\text { Quality of Life In- } \\
\text { dex }\end{array}$ & \multicolumn{4}{|c|}{$\begin{array}{l}\text { Kaplan } 2009 \text { used the Gastro-intestinal Quality of Life Index questionnaire and found significant differences in } \\
\text { favour of laparoscopic appendectomy } 6 \text { weeks and } 6 \text { months after surgery (each, } P<0.05 \text { ). }\end{array}$} & $\begin{array}{l}\oplus \oplus \ominus \ominus \\
\text { low }^{5}\end{array}$ & \\
\hline
\end{tabular}

*The basis for the assumed risk (e.g. the median control group risk across studies) is provided in footnotes. The corresponding risk (and its $95 \%$ confidence interval) is based on the assumed risk in the comparison group and the relative effect of the intervention (and its $95 \% \mathrm{Cl}$ ).

Cl: Confidence interval; Peto OR: Peto Odds ratio

GRADE Working Group grades of evidence

High quality: Further research is very unlikely to change our confidence in the estimate of effect.

Moderate quality: Further research is likely to have an important impact on our confidence in the estimate of effect and may change the estimate. 
Low quality: Further research is very likely to have an important impact on our confidence in the estimate of effect and is likely to change the estimate. Very low quality: We are very uncertain about the estimate.

1 Downgraded two levels for risk of bias (selection and detection bias) and inconsistency (heterogeneity)

2 Downgraded one level for risk of bias (selection and detection bias)

3 Downgraded two levels for risk of bias (selection and detection bias) and imprecision (wide confidence interval)

4 Downgraded three levels for risk of bias (selection and detection bias), inconsistency (heterogeneity), and imprecision (wide confidence interval)

5 Downgraded two levels for risk of bias (selection and detection bias) and imprecision (small sample size)

\section{Summary of findings 4. Subgroup analysis (publication date) - Laparoscopic versus open appendectomy in children for suspected appendicitis}

\section{Subgroup analysis (publication date) - Laparoscopic versus open appendectomy in children for suspected appendicitis}

Patient or population: patients with suspected appendicitis

Settings: most trials were single institutions across Europe, Asia, North America, South America, Australia and South Africa (frequency in descending order) Intervention: subgroup analysis (publication date) - laparoscopic versus open appendectomy in children

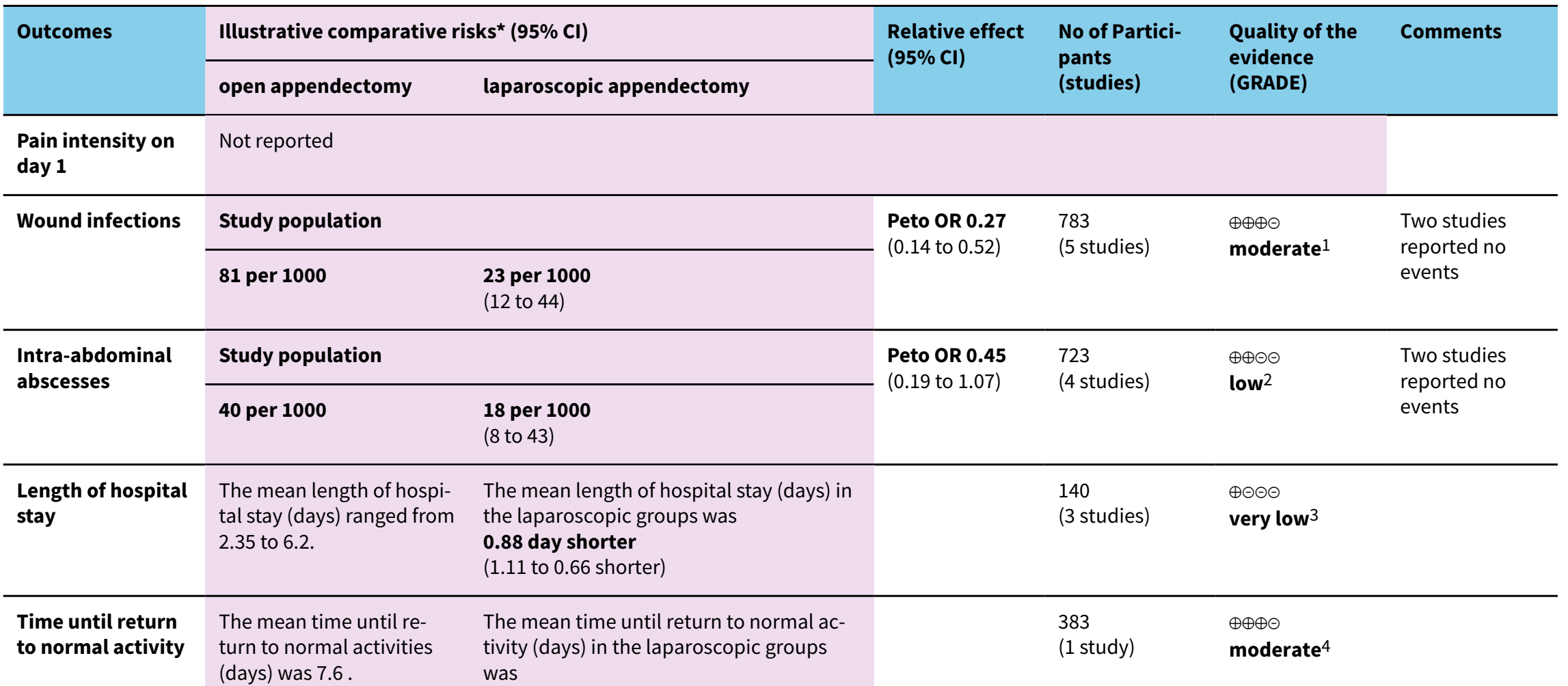


*The basis for the assumed risk (e.g. the median control group risk across studies) is provided in footnotes. The corresponding risk (and its $95 \%$ confidence interval) is based on the assumed risk in the comparison group and the relative effect of the intervention (and its $95 \% \mathrm{Cl}$ ).

Cl: Confidence interval; Peto OR: Peto Odds ratio

GRADE Working Group grades of evidence

High quality: Further research is very unlikely to change our confidence in the estimate of effect.

Moderate quality: Further research is likely to have an important impact on our confidence in the estimate of effect and may change the estimate.

Low quality: Further research is very likely to have an important impact on our confidence in the estimate of effect and is likely to change the estimate.

Very low quality: We are very uncertain about the estimate.

1 Downgraded one level for risk of bias (selection and detection bias)

2 Downgraded two levels for risk of bias (selection and detection bias) and imprecision (wide confidence interval)

3 Downgraded three levels for risk of bias (selection and detection bias), inconsistency (heterogeneity), and imprecision (small sample size)

4 Downgraded one level for risk of bias imprecision (wide confidence interval) 


\section{B A C K G R O U N D}

\section{Description of the condition}

Acute appendicitis is an inflammation of the appendix and it is the most common cause of acute abdominal pain (Cheng 2015; Rehman 2011; Wilms 2011), with an individual lifetime incidence ranging from $6 \%$ to $16 \%$ (Addiss 1990; Lee 2010). The mortality rate following appendectomy (surgical removal of the appendix) is less than 1\% (Andreu-Ballester 2009; Bregendahl 2013; Kotaluoto 2017; Lin 2015; Weiser 2015).

\section{Description of the intervention}

Since the first recorded appendectomy performed by Claudius Amyand in 1735 and its description by McBurney in 1894, appendectomy is the treatment of choice for acute appendicitis (McBurney 1894). It soon became one of the most frequently performed surgical procedures. The surgical technique remained nearly unchanged for over a century, as it combines therapeutic efficacy with low morbidity (diseases affecting the health conditions) and mortality rates (Eriksson 1995). The evolution of endoscopic (using an instrument to look inside the body) surgery led to the idea of performing appendectomy via laparoscopy (surgery using small incisions), which was first described by Semm in 1983 (Semm 1983). In the past, the new method had only partly gained acceptance (Faiz 2008; Paterson 2008; Van Hove 2008). While some studies claimed laparoscopic appendectomy to be superior to open appendectomy in terms of a quicker and less painful recovery, fewer postoperative complications, and better cosmesis (appearance of the incisions after surgery), other studies found no such advantages or even favoured the open approach. Nowadays, laparoscopic appendectomy has gained wide acceptance (Bulian 2013; Ingraham 2010; Van Rossem 2016). It may happen that a non-inflamed appendix is discovered during surgery. Therefore, preoperative imaging techniques are essential for confirming a suspected appendicitis (Webb 2011) because postoperative complications also occur after negative appendectomies (Jeon 2017).

\section{How the intervention might work}

Laparoscopic appendectomy offers several theoretical advantages compared to open appendectomy. Due to smaller incisions, it may be associated with reduced postsurgical pain, a lower wound infection rate and faster return to normal activity.

\section{Why it is important to do this review}

Laparoscopic appendectomy has gained wide acceptance and is the treatment of choice for acute appendicitis. Therefore, the superiority of laparoscopic appendectomy compared to open appendectomy should be established by performing a systematic review based on randomised controlled trials.

\section{OB JECTIVES}

To compare the effects of laparoscopic appendectomy (LA) and open appendectomy $(\mathrm{OA})$ with regard to benefits and harms.

\section{METHODS}

\section{Criteria for considering studies for this review}

Types of studies

We included all randomised (including cluster-randomised) controlled trials comparing laparoscopic surgery and open appendectomy for acute appendicitis. Quasi-randomised trials that allocated participants depending on the availability of staff or instruments or on the number of the day (odd or even) were excluded from the analysis. Due to the large number of studies available, we decided to also exclude trials that had no concealment of allocation.

If a trial was reported only as an abstract, or if no standard deviation was given for an outcome variable, or if the method of randomisation was not stated, the authors were contacted to provide full details of their trial. If the authors of an abstract did not provide information to determine whether it met the inclusion criteria, the abstract was excluded from further analysis.

\section{Types of participants}

We included all participants regardless of age with symptoms and signs of acute appendicitis. If a study reported a rate of more than $50 \%$ of appendix specimens without histological signs of inflammation, we assumed that this study mainly dealt with incidental appendectomies. Therefore, these studies were not included.

\section{Types of interventions}

We performed comparisons of laparoscopic versus open appendectomy in adults and in children.

The classical muscle-splitting appendectomy and further open approaches were compared with laparoscopic appendectomy. Usually, this technique requires the insertion of three trocars into the abdominal cavity (Baird 2017). The appendix can be dissected by using either a stapling device (EndoGIA) or ligatures, the socalled Roeder or EndoLoops. Both techniques can be seen as comparable (Mannu 2017).

\section{Types of outcome measures}

\section{Primary outcomes}

(1) Pain intensity on day one (any validated score such as visual analogue scale);

(2) Wound infections (defined by the study authors as a rate) up to 14 days after surgery;

(3) Intra-abdominal abscesses (defined by the study authors as a rate) up to 14 days after surgery.

\section{Secondary outcomes}

(4) Length of hospital stay (days);

(5) Time until return to normal activities (days);

(6) Quality of life (any validated score, such as SF-36 questionnaire) up to one year after surgery.

\section{Search methods for identification of studies}

\section{Electronic searches}

We conducted a comprehensive literature search to identify published and unpublished randomised controlled trials with 
no language restrictions. The Colorectal Cancer Group (CCCG) performed the searches in the following electronic databases:

- Cochrane Central Register of Controlled Trials (CENTRAL, Issue 2, 2018) (The Cochrane Library) (Appendix 1);

- Ovid MEDLINE (1950 to 9 February 2018) (Appendix 2);

- Ovid Embase (1974 to 9 February 2018) (Appendix 3).

We also searched the following clinical trial registers:

- World Health Organization (WHO) ICTRP portal (9 February 2018);

- EU Clinical Trials Register (9 February 2018);

- ClinicalTrials.gov (9 February 2018).

\section{Searching other resources}

We screened the reference lists of all relevant primary studies and review article for further studies (ANDEM 1997; Aziz 2006; Bennett 2007; Chung 1999; Dai 2017; Fingerhut 1999; Garbutt 1999; Golub 1998; Jaschinski 2015, Kapischke 2006; Kim 2004; Li 2010; Liu 2010; Meynaud-Kraemer 1999; Ohtani 2012, Sadr-Azodi 2009; Slim 1998; Temple 1999; Ukai 2016; Wei 2011). Additionally, authors of relevant articles and known international experts in the field of laparoscopic surgery were contacted to obtain information on any past, present, or future studies.

We handsearched abstracts presented to the following international scientific societies (February 2018): European Association of Endoscopic Surgery (EAES), Society of American Gastrointestinal Endoscopic Surgeons (SAGES), American Society for Gastrointestinal Endoscopy (ASGE), Asian Surgical Association (ASA), International Society for Surgery (ISS), Endoscopic and Laparoscopic Society of Asia (ELSA), Surgical Infection Society (SIS), and the German Society for Surgery (Deutsche Gesellschaft für Chirurgie, DGCh).

\section{Data collection and analysis}

We conducted the review according to the recommendations of the Cochrane Handbook for Systematic Reviews of Interventions (Higgins 2011). We used Review Manager 5.3 software (Review Manager 2014).

\section{Selection of studies}

At least two review authors assessed all studies (CM, MEI, StS, $\mathrm{TJ}$ ) and read the titles and abstracts independently from each other. After the retrieval of potentially relevant studies, review authors independently assessed the full text of the papers to determine whether they met the inclusion criteria for this review. We resolved disagreements by consultation with a third review author. We contacted original study authors if the available data were insufficient to permit a judgement on inclusion.

\section{Data extraction and management}

Two review authors independently extracted the relevant data (CM, StS, TJ). We resolved any differences by discussion. Data from the included studies were entered in the table Characteristics of included studies. We listed excluded studies with reasons for their exclusion in the table Characteristics of excluded studies.

\section{Assessment of risk of bias in included studies}

We used the Cochrane 'Risk of bias' tool for assessing the risk of bias of the included studies (Chapter 8, Higgins 2011). Two review authors assessed the risk of bias of the following domains (CM, StS, TJ):

- Random sequence generation;

- Allocation concealment;

- Blinding of participants and personnel;

- Blinding of outcome assessment;

- Incomplete outcome data;

- Selective reporting bias; and

- Evidence of learning curve bias that might influence the internal validity of trial results.

We judged each domain as low risk, high risk, or unclear risk of bias according to criteria used in the Cochrane 'Risk of bias' tool (see Appendix 4) (Chapter 8.5.d, Higgins 2011).

\section{Measures of treatment effect}

We performed analyses in Review Manager 5.3. provided by Cochrane (Review Manager 2014). We calculated Peto odds ratio (OR) with $95 \%$ confidence intervals (Cls) for dichotomous outcomes, and mean differences (MDs) and 95\% Cls for continuous outcomes. For continuous outcomes, such as quality of life based on different measurement scales, we calculated standardised mean differences (SMDs) with 95\% Cls.

\section{Unit of analysis issues}

We used individual participants as the unit of analysis. We did not identify any cluster-randomised trials. We will include these trials in subsequent versions of this review by using an estimate of the intracluster correlation co-efficient (ICC) according to the Cochrane Handbook for Systematic Reviews of Interventions (Chapter 16.3, Higgins 2011).

\section{Dealing with missing data}

We contacted authors when key information was missing. If missing data became available, we included them in the analysis. If a study failed to report the standard deviation (SD) for an outcome measure, we used the mean of the trial as value for the SD (Follmann 1992). This approach produces relatively conservative results, since studies that did not report SDs tended to receive less weight.

\section{Assessment of heterogeneity}

Clinical heterogeneity caused by differences in participant characteristics and surgeons is likely to be moderate as studies had different inclusion criteria and some authors admitted the presence of a learning curve bias. The presence of statistical heterogeneity of treatment effects among trials was determined using the $\mathrm{Chi}^{2}$ test and the $\mathrm{I}^{2}$ statistic. If a P value was less than 0.05 in the $\mathrm{Chi}^{2}$ test, we explored the causes of heterogeneity by conducting subgroup analyses. .

We classified the degree of heterogeneity as follows (Deeks 2011):

- $0 \%$ to $40 \%$ : might not be important;

- $30 \%$ to $60 \%$ : may represent moderate heterogeneity ${ }^{\star}$; 
- $50 \%$ to $90 \%$ : may represent substantial heterogeneity ${ }^{\star}$;

- $75 \%$ to $100 \%$ : considerable heterogeneity ${ }^{*}$.

*The importance of the observed value of $1^{2}$ depends on (i) magnitude and direction of effects and (ii) strength of evidence for heterogeneity (e.g. P value from the chi-squared test, or a confidence interval for 12 ).

\section{Assessment of reporting biases}

To control for the influence of a possible publication bias, we tested funnel plot asymmetry, as proposed by Egger and colleagues (Egger 1997) for comparisons that included at least 10 trials.

\section{Data synthesis}

We performed statistical analyses by using Review Manager 5.3 software (Review Manager 2014). After considering details from the Characteristics of included studies table, we assumed that the true treatment effect might differ between studies because of differences in participants, surgical skills, and outcome measurements. Therefore, we preferred the random-effects model. However, we used the fixed-effects model to calculate the Peto odds ratio. We considered a $P$ value less than 0.05 to be statistically significant. We presented the results of meta-analyses for each outcome graphically as forest plots.

\section{Subgroup analysis and investigation of heterogeneity}

Studies which evaluated LA in children or one sex only were not excluded, but studies in children were analysed separately. Additionally, we performed subgroup analyses for studies published within the last decade (from 2007), in order to consider the changes in laparoscopy and imaging.

\section{Sensitivity analysis}

Following sensitivity analyses were planned:

- Including only studies with all domains at low risk of bias.

\section{Summary of Findings table}

We assessed the quality of evidence using the Grading of Recommendations Assessment, Development and Evaluation (GRADE) approach in 'Summary of Findings' Table(s). In randomised controlled trials, the evidence can be downgraded from 'high quality' by one level for serious (or by two levels for very serious) limitations, depending on assessments for risk of bias, inconsistency of results, indirectness of evidence, imprecision or publication bias. Therefore, the evidence of randomised controlled trials could be downgraded to 'moderate' (on level), 'low' (two levels) and 'very low' (three levels).

The GRADE system classifies the quality of evidence in one of four grades:

\begin{tabular}{ll}
\hline Grade & Definition \\
\hline High & Further research is very unlikely to change our confidence in the estimate of effect \\
\hline Moderate & $\begin{array}{l}\text { Further research is likely to have an impact on our confidence in the estimate of effect and may } \\
\text { change the estimate }\end{array}$ \\
\hline Low & $\begin{array}{l}\text { Further research is very likely to have an important impact on our confidence on the estimate of ef- } \\
\text { fect and is likely to change the estimate }\end{array}$
\end{tabular}

Very low

Any estimate of effect is very uncertain

Factors that influence the quality of evidence:

\begin{tabular}{ll}
\hline Downgrades the evidence & Upgrades the evidence \\
\hline Study limitation & Large magnitude of effect \\
\hline Inconsistency of results & All plausible confounding would reduce the demonstrated effect \\
\hline Indirectness of evidence & Dose-response gradient \\
\hline Imprecision & \\
\hline Publication bias & \\
\hline
\end{tabular}




\section{RESULTS}

\section{Description of studies}

See: 'Characteristics of included studies', 'Characteristics of excluded studies' and 'Ongoing studies'.

\section{Results of the search}

Overall, we identified 1607 potentially matching records through database searching until 9 February 2018. We identified 15 further records through handsearching and through scanning reference lists of included studies and relevant reviews. After screening titles and abstracts, we excluded 731 duplicates and 724 irrelevant records. We identified five abstracts and added them to 'Studies awaiting classification' (Barth 1999; Esposito 1997; Hoff 1995; Loh 1992; Rohr 1994). Since the five abstracts are over 15 years old and it has not been possible to obtain further data from the authors, we do not expect any further peer reviewed data. We retrieved the remaining 167 records for further assessment. In total, 85 randomised studies met the inclusion criteria after excluding 82 trials for the reasons listed in the table 'Characteristics of excluded studies'. Additionally, we identified five ongoing studies (see: 'Characteristics of ongoing studies').

Figure 1 presents the Prisma Flow Chart for this update. 
Figure 1. Study flow diagram.

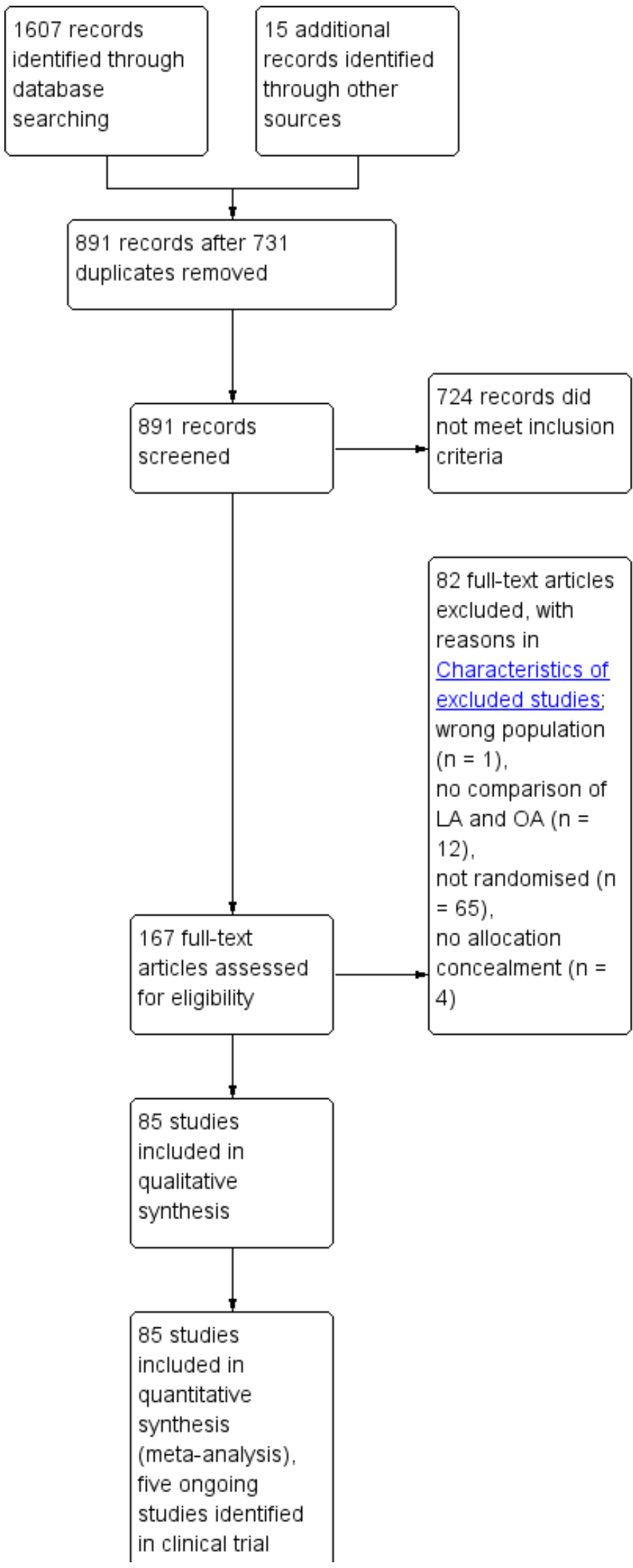


Figure 1. (Continued)

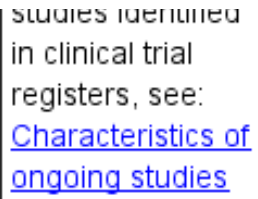

\section{Included studies}

In the 2018 update, we added 18 additional studies (Aktimur 2016; Bartin 2016; Bliss 2014; Cipe 2014; Goudar 2011; Gundavda 2012; Kargar 2011; Khalil 2011; Kocatas 2013; Kouhia 2010; Mahmood 2016; Saha 2010, Schietroma 2012; Singh 2017; Sozutek 2013; Taguchi 2015; Thomson 2015; Yu 2016), which resulted in a total of 85 RCTs, of which 75 compared LA versus OA in adults. An interim analysis (Tzovaras 2007) was replaced by updated results (Tzovaras 2010). We identified no cluster-randomised controlled trials. For further details of the studies, please see: Characteristics of included studies.

All the included studies used similar subjective clinical criteria to identify participants suspected of suffering from acute appendicitis. In most studies, the authors did not specify the clinical diagnosis of acute appendicitis. Only 11 trials used routine preoperative imaging techniques. Ultrasonography was performed in five trials (Goudar 2011; Hebebrand 1994; Khalil 2011; Mahmood 2016; Singh 2017) and ultrasonography or computed tomography were used in three studies (Cipe 2014; Kaplan 2009; Sozutek 2013). The authors of two trials did not define the radiological investigation (Kocatas 2013; Thomson 2015). Furthermore, abdominal imaging with either ultrasonography or computed tomography (CT) scan were selectively verified in one study (Tzovaras 2010).

The age range and the gender ratio, however, were quite different among the studies. Some of the studies excluded patients in whom a perforated appendix was suspected. Although this was not explicitly described in all studies, we assumed that all studies excluded pregnant women and patients in whom laparoscopy was preoperatively believed impractical.

The interventions were very similar among the studies. Laparoscopic appendectomy was usually performed with the use of three trocars for access. The appendix was dissected by using ligatures in 33 studies (39\%), a stapling device for securing the appendix stump was routinely used in 13 trials (15\%) and different techniques in 12 trials (14\%). The remaining 27 studies (32\%) did not describe the dissection. The trial by Ortega and colleagues (Ortega 1996) had a three-armed design to compare stapling and loops for appendiceal stump closure. While some trials used disposable instruments (which are quite expensive), others used reusable ones. One further three-armed trial used needlescopic instruments with $2 \mathrm{~mm}$ ports (Huang 2001). Nearly all studies reported that both treatment groups received the same single dose of antibiotics.

There were 10 multicentre studies, of which only one used standardised operative techniques (Bliss 2014). Five authors reported differences in the performing of LA (Hellberg 1999; Kazemier 1997; Lavonius 2001; Long 2000; Ortega 1996) and an assessment of four trials was not possible because of insufficient reporting (Helmy 2001; Kald 1999; Katkhouda 2005; Pedersen 2001).

There was almost no information on the time of randomisation. Five trials performed randomisation in the operating room (Bliss 2014; Hart 1996; Lejus 1996; Little 2002; Tzovaras 2010).

Authors did not specify the follow-up in 28 of 85 trials (33\%). Study participants were followed up until discharge in three studies, over the first postoperative week in seven studies, between two and three weeks in seven trials and until the return to work in four trials. The authors of 25 trials (29\%) reported a follow-up of one month. The follow-up ranged from two to three months in five studies (6\%). There were studies with a follow-up of six months (Kaplan 2009; Schietroma 2012; Schippers 1997), 12 months (Long 2000) and six to 26 months (Henle 1996). The only study with a long-term followup of nearly 10 years was undertaken by Van Dalen who reviewed seven of 63 participants with their appendix still in situ after 10 years (Van Dalen 2003). However, there were no long-term data available to describe the effects of LA and OA on the occurrence of late complications, such as incisional hernia or intra-abdominal adhesions.

Of the 85 trials, 40 (47\%) were conducted in Europe, 28 (33\%) in Asia, 11 (13\%) in North America, one in South America, three in Australia, and two in South Africa. Eleven of the included studies were performed in the USA (the highest number of studies performed in a single country). For further details of the countries of origin, please see Characteristics of included studies.

Most trials assessed several clinical outcomes within the hospital stay. Outcomes, which were not assessed in this review (but in were assessed in some primary trials), were anaesthesia duration (Al-Mulhim 2002; Katkhouda 2005; Mutter 1996; Schippers 1997; Yin 1996), time until reintroduction of liquid diet (Al-Mulhim 2002; Kaiser 2006; Katkhouda 2005; Kazemier 1997; Kum 1993a; Sun 1998; Tate 1993a), cosmesis (Kaiser 2006; Schippers 1997; Pedersen 2001); costs (Heikkinen 1998; Kald 1999; Kaplan 2009; Long 2000; Martin 1995; Minné 1997; Wei 2010; Williams 1996; Yin 1996), the rate of bacteraemia (Sezeur 1997; Nordentoft 2000), the rate of postoperative adhesions on second-look laparoscopy (DeWilde 1991), and some pathophysiological parameters (Perner 1999; Karadayi 2003; Simon 2009).

Three trials presented their results in combination with other results. One trial compared laparoscopic appendectomy and cholecystectomy against the corresponding open techniques (Settmacher 1995). A second very small trial reported on LA and OA, but also on laparoscopic colectomy $(n=11$; Perner 1999). A third trial reported results for randomised and nonrandomised participants (Williams 1996). In all these cases, we used only the relevant data. 
One trial was described only in a meta-analysis, of which the primary trialist was a co-author (Barth 1999). We met the trialist, but he failed to provide us with further data. We decided to include the trial results that were reported in the meta-analysis.

Eight trials were reported in more than one publication (Hellberg 1999; Henle 1996; Katkhouda 2005; Kazemier 1997; Lintula 2004; Minné 1997; Olsen 1993; Tzovaras 2010). Two articles (Lintula 2004; Tzovaras 2010) described different stages of the same trial. We used the most complete information, wherever possible. One trial was reported in three different publications (Henle 1996). We were unable to resolve the discrepancies in complication rates among the three papers (Neugebauer 1999). We decided not to use the trial results on infectious complications, but we included the trial's other outcome data, which were reported consistently.

\section{Excluded studies}

In total, we excluded 82 studies; one study enrolled the wrong population, 12 studies did not compare LA with OA, 65 trials were not randomised, and four studies were without allocation concealment. See Characteristics of excluded studies.

\section{Risk of bias in included studies}

We describe the 'Risk of bias' in the Characteristics of included studies section. We used the Cochrane 'Risk of bias tool' to assess the methodological quality of the trials (see Figure 2 and Figure 3). Of the included trials, only one had a low risk of bias on all domains (Moberg 2005). Therefore, we did not perform any sensitivity analyses. The largest risk of bias was attrition bias due to incomplete outcome data. The smallest risk of bias was selection bias due to adequate allocation concealment. 
Figure 2. Methodological quality summary: review authors' judgements about each methodological quality item for each included study.

\begin{tabular}{|c|c|c|c|c|c|c|c|}
\hline & 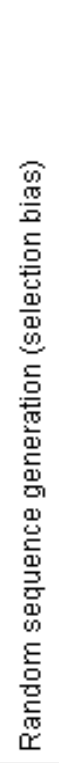 & 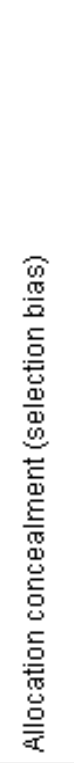 & 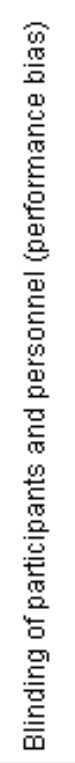 & 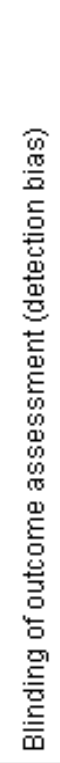 & 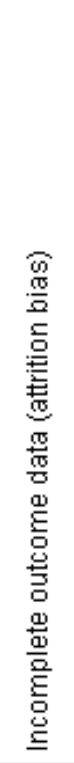 & 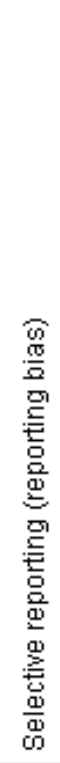 & 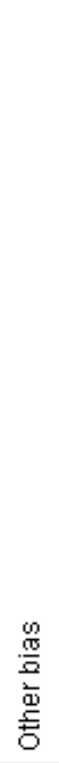 \\
\hline Aktimur 2016 & + & + & $?$ & $?$ & $?$ & $?$ & + \\
\hline Al-Mulhim 2002 & $?$ & $\odot$ & $?$ & $?$ & $?$ & $?$ & + \\
\hline Attwood 1992 & $?$ & + & $?$ & $?$ & + & $?$ & $\Theta$ \\
\hline Barth 1999 & $?$ & $?$ & $?$ & $?$ & $?$ & $?$ & $?$ \\
\hline Bartin 2016 & $?$ & $?$ & $?$ & $?$ & + & $?$ & $?$ \\
\hline Bauwens 1999 & $?$ & $\odot$ & $?$ & $?$ & - & + & $\oplus$ \\
\hline Bliss 2014 & + & + & + & + & + & + & $?$ \\
\hline Bolla 2008 & $?$ & $?$ & $?$ & $?$ & $?$ & $?$ & $?$ \\
\hline Bruwer 2000 & $\odot$ & $\odot$ & $?$ & $?$ & + & $?$ & $\oplus$ \\
\hline Cipe 2014 & + & $?$ & $?$ & $?$ & $\odot$ & $?$ & $?$ \\
\hline Cox 1996 & $?$ & $?$ & $?$ & $?$ & $?$ & $?$ & $\odot$ \\
\hline Dewilde 1991 & $?$ & $?$ & $?$ & $?$ & $?$ & $?$ & $?$ \\
\hline Eichen 1994 & $\oplus$ & + & $?$ & $?$ & $?$ & $?$ & $\oplus$ \\
\hline Frazee 1994 & $?$ & $?$ & $?$ & $?$ & 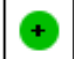 & $?$ & $\odot$ \\
\hline Goudar 2011 & $?$ & $?$ & $O$ & $\theta$ & - & $\theta$ & $?$ \\
\hline Gundayda 2012 & $?$ & $?$ & $?$ & $?$ & $?$ & $?$ & $?$ \\
\hline Hansen 1996 & + & + & $?$ & $?$ & $\odot$ & $?$ & + \\
\hline Hart 1996 & + & + & $?$ & $?$ & O & + & + \\
\hline Hebebrand 1994 & $?$ & + & $\odot$ & 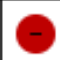 & + & + & $\boldsymbol{P}$ \\
\hline Heikkinen 1998 & $?$ & + & $?$ & $?$ & $?$ & $?$ & $\odot$ \\
\hline
\end{tabular}


Figure 2. (Continued)

\begin{tabular}{|c|c|c|c|c|c|c|c|}
\hline Heikkinen 1998 & $?$ & + & $?$ & $?$ & $?$ & $?$ & $\oplus$ \\
\hline Hellberg 1999 & $\odot$ & + & $?$ & $?$ & 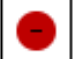 & $?$ & $\oplus$ \\
\hline Helmy 2001 & $?$ & $?$ & $?$ & $?$ & $?$ & $?$ & $\oplus$ \\
\hline Henle 1996 & $\odot$ & $?$ & $?$ & $?$ & $\odot$ & $?$ & $\odot$ \\
\hline Huang 2001 & $?$ & + & $?$ & $\odot$ & $?$ & $?$ & + \\
\hline Ignacio 2003 & $\odot$ & + & $\odot$ & $?$ & + & $?$ & + \\
\hline Jadallah 1994 & $?$ & + & $?$ & $?$ & + & $?$ & $?$ \\
\hline Kaiser 2006 & $?$ & + & $?$ & $?$ & + & + & $\odot$ \\
\hline Kald 1999 & $\odot$ & + & $?$ & $?$ & $\odot$ & + & $\oplus$ \\
\hline Kaplan 2009 & $?$ & $?$ & $?$ & $?$ & + & $?$ & $\odot$ \\
\hline Karadayi 2003 & $\odot$ & $?$ & $?$ & $?$ & $\odot$ & + & + \\
\hline Kargar 2011 & $\odot$ & $\odot$ & $\odot$ & $\odot$ & $\odot$ & $?$ & $?$ \\
\hline Katkhouda 2005 & $\odot$ & + & $\odot$ & $\odot$ & $\odot$ & $\oplus$ & $\oplus$ \\
\hline Kazemier 1997 & $\odot$ & 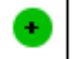 & $?$ & $?$ & + & $?$ & + \\
\hline Kehagias 2009 & $\odot$ & + & $?$ & $?$ & $?$ & $?$ & $?$ \\
\hline Khalil 2011 & $?$ & $?$ & $\Theta$ & $\odot$ & 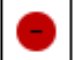 & $?$ & $\odot$ \\
\hline Kocatas 2013 & + & $\odot$ & $?$ & $?$ & $?$ & + & + \\
\hline Kouhia 2010 & $?$ & + & $?$ & $?$ & 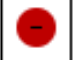 & $?$ & $\odot$ \\
\hline Kum 1993a & + & + & $?$ & $?$ & 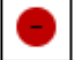 & $?$ & + \\
\hline Laine 1997 & $?$ & $?$ & $?$ & $?$ & $\odot$ & $?$ & $\odot$ \\
\hline Larsson 2001 & $?$ & + & $?$ & $?$ & $O$ & $?$ & $\odot$ \\
\hline Lavonius 2001 & $?$ & + & $?$ & $?$ & + & $?$ & $\oplus$ \\
\hline Lejus 1996 & $?$ & $?$ & + & $?$ & + & $?$ & $?$ \\
\hline Lintula 2004 & $?$ & + & $?$ & $?$ & $\odot$ & $?$ & $\oplus$ \\
\hline Little 2002 & $?$ & + & $?$ & $?$ & + & $?$ & $\odot$ \\
\hline Long 2000 & + & $\odot$ & $?$ & $?$ & $?$ & $?$ & + \\
\hline Macarulla 1995 & $?$ & + & $?$ & $?$ & + & $?$ & $\odot$ \\
\hline Mahmood 2016 & $?$ & $?$ & $?$ & $?$ & + & $?$ & $\oplus$ \\
\hline Martin 1995 & $?$ & $?$ & $?$ & $?$ & + & $?$ & $?$ \\
\hline Minné 1997 & $\odot$ & $?$ & $?$ & $?$ & - & $?$ & 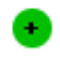 \\
\hline Moberg 2005 & $\odot$ & $\odot$ & $\odot$ & $\odot$ & $\odot$ & $\odot$ & $\odot$ \\
\hline
\end{tabular}


Figure 2. (Continued)

\begin{tabular}{|c|c|c|c|c|c|c|c|}
\hline Moberg 2005 & $\odot$ & + & $\oplus$ & + & + & + & $\odot$ \\
\hline Moirangthem 2008 & $?$ & $?$ & $?$ & $?$ & $\odot$ & $?$ & $?$ \\
\hline Mutter 1996 & $?$ & $?$ & $?$ & $?$ & + & $?$ & $\odot$ \\
\hline Navarra 2000 & $\odot$ & + & $?$ & $?$ & + & $?$ & $?$ \\
\hline Nordentoft 2000 & $?$ & $?$ & $?$ & $?$ & $\odot$ & $?$ & $?$ \\
\hline Olsen 1993 & $?$ & $?$ & $?$ & $?$ & + & $?$ & + \\
\hline Ortega 1996 & + & + & + & + & + & $?$ & + \\
\hline Özmen 1999 & $?$ & $?$ & $?$ & $?$ & $?$ & $?$ & $?$ \\
\hline Pedersen 2001 & $\odot$ & + & $?$ & $?$ & $?$ & + & $\odot$ \\
\hline Perner 1999 & $?$ & + & $?$ & $?$ & $?$ & $?$ & $?$ \\
\hline Pozo 1996 & $?$ & $?$ & $?$ & $?$ & $\odot$ & $?$ & $\odot$ \\
\hline Reiertsen 1997 & $?$ & $?$ & $\odot$ & $\odot$ & 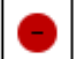 & $?$ & $\odot$ \\
\hline Ricca 2007 & $\odot$ & + & $\odot$ & $?$ & + & $?$ & $\odot$ \\
\hline Saha 2010 & $\odot$ & + & $?$ & $?$ & + & $?$ & $?$ \\
\hline Schietroma 2012 & $\odot$ & $?$ & $?$ & $?$ & 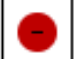 & $?$ & $?$ \\
\hline Schippers 1997 & $?$ & $\odot$ & $?$ & $?$ & + & $?$ & $\odot$ \\
\hline Settmacher 1995 & $?$ & + & $?$ & $?$ & $?$ & $\odot$ & $?$ \\
\hline Sezeur 1997 & $?$ & $\odot$ & $?$ & $?$ & 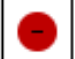 & $?$ & $?$ \\
\hline Simon 2009 & $\odot$ & $?$ & $?$ & $?$ & 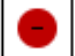 & $?$ & $?$ \\
\hline Singh 2017 & $\odot$ & $?$ & $\odot$ & $\odot$ & + & $?$ & $?$ \\
\hline Sozutek 2013 & $\odot$ & + & $?$ & $?$ & + & + & $\odot$ \\
\hline Stare 1998 & $?$ & + & $\odot$ & $\odot$ & $\odot$ & $?$ & $\odot$ \\
\hline Sun 1998 & $?$ & $?$ & $?$ & $?$ & + & $?$ & $?$ \\
\hline Taguchi 2015 & $\odot$ & + & $\odot$ & $\odot$ & + & $?$ & $\odot$ \\
\hline Tate 1993a & + & + & $?$ & $?$ & + & $?$ & $\odot$ \\
\hline Thomson 2015 & $\odot$ & + & $?$ & $?$ & $\odot$ & $?$ & $\odot$ \\
\hline Tzovaras 2010 & $\odot$ & $\odot$ & $?$ & $?$ & + & + & $\odot$ \\
\hline Vallribera 2003 & $\odot$ & $\odot$ & $?$ & $?$ & $\odot$ & $?$ & $\odot$ \\
\hline Van Dalen 2003 & $?$ & + & $?$ & $?$ & + & $?$ & $?$ \\
\hline Wei 2010 & $?$ & $?$ & $?$ & $?$ & + & $?$ & + \\
\hline Williams 1996 & $?$ & $?$ & $?$ & $?$ & $\theta$ & $?$ & $?$ \\
\hline
\end{tabular}


Figure 2. (Continued)

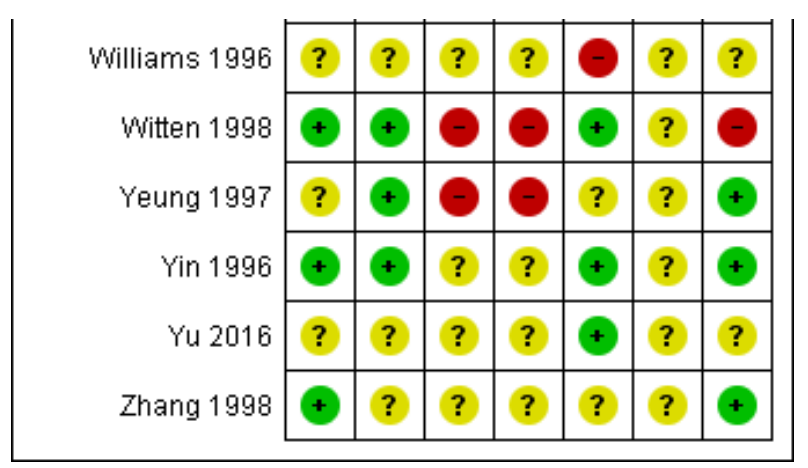

Figure 3. Risk of bias graph: review authors' judgements about each risk of bias item presented as percentages across all included studies.

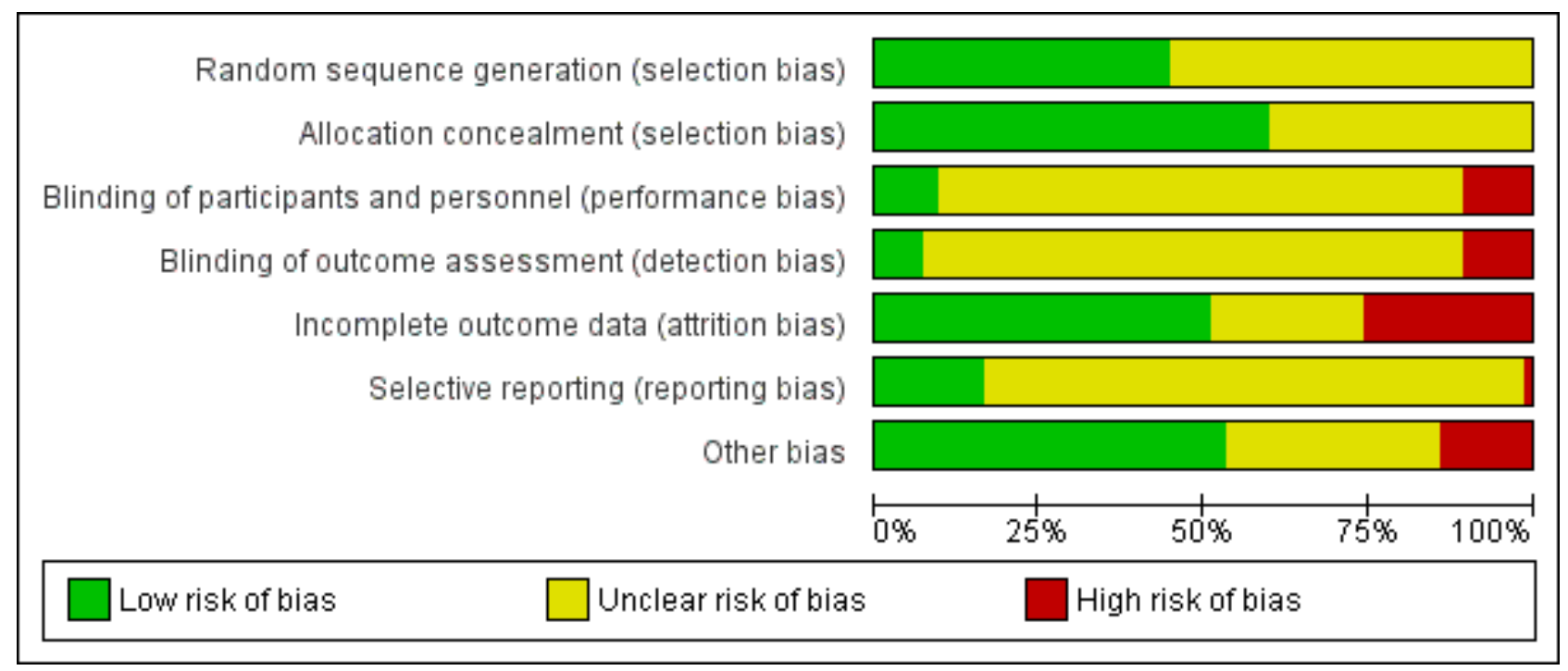

\section{Allocation}

Thirty-eight trials (45\%) had low risk of bias for random sequence generation and 47 trials (55\%) had unclear risk of bias for this domain. The large number of trials allowed us to exclude the trials without adequate allocation concealment. In total, we judged 51 trials $(60 \%)$ to have an adequately concealed process of randomisation.

\section{Blinding}

Not unexpectedly, only 11 trials took measures to blind investigator and/or participant and/or outcome assessor against treatment received (Bliss 2014; Huang 2001; Ignacio 2003; Katkhouda 2005; Lejus 1996; Lintula 2004; Moberg 2005; Ortega 1996; Ricca 2007; Singh 2017; Yin 1996). This represents 13\% of all trials. However, only five studies provided a detailed description of blinding methods (Bliss 2014; Katkhouda 2005; Moberg 2005; Ortega 1996; Singh 2017).

\section{Incomplete outcome data}

In nearly all studies, protocol violations occurred, which subsequently were or were not analysed on an intention-to-treat
(ITT) basis. Participants who were intraoperatively converted from LA to OA (or vice versa) were excluded from analysis in eight studies (Bauwens 1999; Goudar 2011; Hart 1996; Henle 1996; Karadayi 2003; Nordentoft 2000; Schietroma 2012; Williams 1996) and analysed separately in seven studies (Hansen 1996; Hebebrand 1994; Heikkinen 1998; Jadallah 1994; Navarra 2000; Ortega 1996; Witten 1998). In the latter group, we were able to calculate ITT data. As a results of requests, authors provided data for all randomised participants according to the ITT principle (Cipe 2014). However, other participant subgroups, such as patients with appendicitis but no visible perforation (Schietroma 2012), with perforated appendicitis (Huang 2001; Karadayi 2003; Kum 1993a; Simon 2009), a histologically normal appendix (Huang 2001; Karadayi 2003; Kum 1993a; Nordentoft 2000; Reiertsen 1997; Thomson 2015 ), conversion to midline laparotomy (Huang 2001; Kald 1999, Nordentoft 2000; Reiertsen 1997; Sezeur 1997; Williams 1996), nonprotocol medication (Minné 1997; Nordentoft 2000; Sezeur 1997), or inadequate follow-up period (Kald 1999; Khalil 2011; Kocatas 2013; Ortega 1996) were also excluded after randomisation in some of the trials. In total, we were able to use ITT data from 43 of 85 studies (51\%). 


\section{Selective reporting}

The trial protocols were not available for any of the trials. We assessed 14 studies (16\%) as having a low risk of selective reporting bias, $70(82 \%)$ as having an unclear risk of bias for this item, and one study $(1 \%)$ as having high risk of bias.

\section{Other potential sources of bias}

We could rule out differential expertise bias in 45 trials (53\%), and in 12 trials (14\%) the trialists admitted the presence of a learning curve bias. In turn, this means that 28 trial reports (33\%) failed to discuss the importance of surgical expertise for the results of the trial.

\section{Effects of interventions}

See: Summary of findings for the main comparison Laparoscopic versus open appendectomy in adults or adolescents for suspected appendicitis; Summary of findings $\mathbf{2}$ Laparoscopic versus open appendectomy in children for suspected appendicitis; Summary of findings 3 Subgroup analysis (publication date) - Laparoscopic versus open appendectomy in adults or adolescents for suspected appendicitis; Summary of findings 4 Subgroup analysis (publication date) - Laparoscopic versus open appendectomy in children for suspected appendicitis

See Summary of findings for the main comparison for adults and Summary of findings 2 for children.

\section{Laparoscopic versus open appendectomy in adults or adolescents}

\section{Primary outcomes}

\section{Pain intensity on day one}

Pain measurements at day one after surgery showed a reduction of pain by $0.75 \mathrm{~cm}$ on a $10 \mathrm{~cm}$-VAS for LA (MD $-0.75 \mathrm{~cm}, 95 \% \mathrm{Cl}$ -0.45 to $-1.04 ; 20$ RCTs; 2421 participants; $1^{2}=74 \%$; low-quality evidence; Analysis 1.1). This finding is hampered by the fact that considerable heterogeneity was found among the studies and also the absolute pain levels varied between $5.9 \mathrm{~cm}$ and $1.4 \mathrm{~cm}$. Less pain was also found in most of those trials that measured pain over one or two weeks after surgery (Bauwens 1999; Gundavda 2012; Hart 1996; Hebebrand 1994; Hellberg 1999; Katkhouda 2005; Moberg 2005; Reiertsen 1997; Ricca 2007), although the absolute differences became smaller with time. Various studies reported that LA required fewer analgesic drug doses, a shorter duration of analgesia, or both. When restricting the analysis of pain intensity only to the trials that used blinding, the pooled result was still significantly in favour of LA.

Only 24 authors reported analgesia requirements. No difference between LA and OA were reported in 13 studies and a lower consumption of analgesia following LA was observed in 11 trials. Eight studies reported the requirements of analgesia and the pain measurement. All eight trials showed a reduction in postoperative pain for participants following LA. However, the lower pain scores were only statistically significant in four trials (Cipe 2014; Hebebrand 1994; Kazemier 1997; Ortega 1996). Six authors also reported a reduced consumption of analgesia following LA (Cipe 2014; Gundavda 2012; Hebebrand 1994; Kazemier 1997; Singh 2017; Sozutek 2013). The other two authors did not find any difference between LA and OA (Ortega 1996; Tate 1993a). Therefore, three of eight studies showed statistically significant lower pain scores and reduced consumption of analgesia following LA (Cipe 2014;
Hebebrand 1994; Kazemier 1997). The funnel plot showed no signs of publication bias.

The subgroup analysis also showed a reduction of pain by $0.52 \mathrm{~cm}$ on a $10 \mathrm{~cm}$-VAS for LA (MD $-0.52 \mathrm{~cm}, 95 \% \mathrm{Cl}-0.06$ to $-0.98 ; 7$ RCTs; 613 participants; $\left.\right|^{2}=75 \%$; low-quality evidence; Analysis 3.1).

\section{Wound infections}

From the complications that were described after OA and LA, we examined the two most relevant specific complications. It was impossible to extract data on overall complication rates from the included studies because definition and reporting of complications was inconsistent. Wound infections were about half as likely after LA than after OA (Peto OR $0.42,95 \% \mathrm{Cl} 0.35$ to $0.51 ; 63$ RCTs; 7612 participants; $12=7 \%$; moderate-quality evidence; Analysis 1.2), a highly significant result based on over 7,600 operated cases. Despite the large number of studies with differences in surgical expertise, antibiotic regimens, and clinical setting, no heterogeneity was detectable. The funnel plot showed no signs of publication bias.

The subgroup analysis also presented a reduction of wound infections after LA (Peto OR 0.37, 95\% Cl 0.27 to $0.51 ; 19$ RCTs; 2217 participants; $\left.\right|^{2}=8 \%$; moderate-quality evidence; Analysis 3.2 ),

\section{Intra-abdominal abscesses}

On the other hand, intra-abdominal abscesses were increased significantly after LA (Peto OR 1.65, 95\% Cl 1.12 to 2.43; 53 RCTs; 6677 participants; $1^{2}=30 \%$; moderate-quality evidence; Analysis 1.3). In this case, no heterogeneity was detectable among the 53 studies that contributed data to this overall result. The funnel plot showed no signs of publication bias.

By contrast, the subgroup analysis illustrated a trend towards fewer intra-abdominal abscesses after LA (Peto OR $0.82,95 \% \mathrm{Cl} 0.44$ to 1.51; 11 RCTs; 1466 participants; $I^{2}=39 \%$; low-quality evidence; Analysis 3.3).

\section{Secondary outcomes}

\section{Length of hospital stay}

There were large variations among the absolute lengths of hospital stay in the various studies (range one to 12 days) and for the LA versus $\mathrm{OA}$ differences (range 0 to five days reduction). However, a single study reported a significant increase in hospital stay after LA (Kargar 2011). In the summary statistics, a significant reduction of one day following LA was calculated (MD -0.96 days, $95 \% \mathrm{Cl}-1.23$ to $-0.70 ; 46$ RCTs; 5127 participants; $12=95 \%$; low-quality evidence; Analysis 1.4). The fact that study results were highly heterogeneous can be partly explained by the absolute duration of hospital stay, which ranged between one and 12 days. The trials with long hospital stay after OA reported clearly higher reductions in length of stay after LA as compared to those trials, in which hospital stay was already very short after OA. The funnel plot showed no signs of publication bias.

The subgroup analysis also showed a reduced hospital stay after LA (MD -0.84 days, $95 \% \mathrm{Cl}-1.23$ to $-0.44 ; 17$ RCTs; 1977 participants; I $^{2}$ = 97\%; low-quality evidence; Analysis 3.4).

\section{Time until return to normal activity}

Return to normal activity was five days earlier after LA than after OA (MD -4.97 days, $95 \% \mathrm{Cl}-6.77$ to $-3.16 ; 17$ RCTs; 1653 participants; I $^{2}$ $=87 \%$; low-quality evidence; Analysis 1.5 ), but again considerable 
heterogeneity was present. The funnel plot showed no signs of publication bias.

The subgroup analysis presented only a trend towards a reduced time until return to normal after LA (MD -2.27 days, $95 \% \mathrm{Cl}-5.81$ to 1.26; 3 RCTs; 380 participants; 12 = 94\%; very low-quality evidence; Analysis 3.5)

\section{Quality of life}

Two studies reported quality of life using different scales and time points, hindering the possibility of meta-analysis. Kaplan 2009 used the Gastro-intestinal Quality of Life Index questionnaire and found significant differences in favour of LA six weeks and six months after surgery (each $\mathrm{P}<0.05$ ). Katkhouda 2005 used the SF-36 questionnaire two weeks after surgery. The quality of life scores were significantly better in the laparoscopic group for two out of eight domains: physical functioning $(P<0.001)$ and general health $(P=0.018)$. No differences were reported for the remaining six domains: bodily pain, role physical, social functioning, vitality, role emotional, and mental health (no P values stated). We assessed the quality of evidence as low.

Kaplan 2009 was the only study available for the subgroup analysis. We rated the quality of evidence as low.

\section{Laparoscopic versus open appendectomy in children}

\section{Primary outcomes}

\section{Pain intensity on day one}

In children, fewer data were available. Lintula 2004 failed to find a reduction in pain on day one after surgery $(M D-0.80 \mathrm{~cm}, 95 \% \mathrm{Cl}$ -1.65 to $0.05 ; 1 \mathrm{RCT} ; 61$ participants; low-quality evidence; Analysis 2.1).

No study was available for the subgroup analysis.

\section{Wound infections}

Wound infections were significantly reduced following LA (Peto OR $0.25,95 \% \mathrm{Cl} 0.15$ to $0.42 ; 10$ RCTs; 1245 participants; $\left.\right|^{2}=4 \%$; moderate-quality evidence; Analysis 2.2). The funnel plot showed no signs of publication bias.

The subgroup analysis also illustrated fewer wound infections after LA. (Peto OR $0.27,95 \% \mathrm{Cl} 0.14$ to 0.52 ; 5 RCTs; 783 participants; $\left.\right|^{2}=$ $0 \%$; moderate-quality evidence; Analysis 4.1).

\section{Intra-abdominal abscesses}

There was a trend towards decreased intra-abdominal abscesses after LA (Peto OR $0.54,95 \% \mathrm{Cl} 0.24$ to $1.22 ; 9$ RCTs; 1185 participants; $I^{2}=0 \%$; low-quality evidence; Analysis 2.3 ). This finding contradicted the effect in adults and was strongly influenced by two large studies analysing the results of 383 (Bliss 2014) and 260 children (Yu 2016).

The subgroup analysis also showed a trend towards decreased intra-abdominal abscesses after LA (Peto OR 0.45, 95\% Cl 0.19 to 1.07 ; 4 RCTs; 723 participants; $1^{2}=0 \%$; low-quality evidence; Analysis 4.2)

\section{Secondary outcomes}

\section{Length of hospital stay}

The hospital stay was shortened by 0.8 days following LA (MD -0.81 days, $95 \% \mathrm{Cl}-1.01$ to $-0.62 ; 6 \mathrm{RCTs}$; participants $=316$; $\mathrm{I}^{2}=62 \%$; lowquality evidence; Analysis 2.4), but between-trial differences were large.
The subgroup analysis also reported a reduced hospital stay after LA (MD -0.88 days, $95 \% \mathrm{Cl}-1.11$ to -0.66 ; 3 RCTs; participants = 140; $\mathrm{I}^{2}=79 \%$; very low-quality evidence; Analysis 4.4).

\section{Time until return to normal activity}

We found no difference in the return to normal activity (MD -0.50 , $95 \% \mathrm{Cl}-1.30$ to $0.30 ; 1 \mathrm{RCT} ; 383$ participants; moderate-quality evidence; Analysis 2.5). Bliss 2014 was the only study reporting data.

The subgroup analysis (Analysis 4.3) included the same study.

\section{Quality of life}

None of the included studies on children reported this outcome.

\section{DISCUSSION}

\section{Summary of main results}

We included 85 studies involving 9765 participants, of which 75 trials compared LA versus OA in adults. In this update, we included 18 additional studies compared to the previous published version (Sauerland 2010).

The quality of all included studies was moderate to low and most studies had very similar flaws.

In adults, wound infections were less likely after LA than after $O A$ but the incidence of intra-abdominal abscesses was increased. Therefore, the effects of LA are difficult to value because neither LA nor OA is clearly superior to the other approach. However, there was a trend towards decreased intra-abdominal abscesses after LA for the subgroup analysis and in children undergoing LA. Furthermore, it is doubtful whether the extent of the significant reduction of pain on day one after surgery is also clinically relevant. A reduction of pain by $0.75 \mathrm{~cm}$ on a $10 \mathrm{~cm}$-VAS scale - as it was found here - has been shown to be under the level of pain that an average patient is able to perceive. However, the absolute pain intensity and its duration also have to be taken into account as well as the possibly reduced number of analgesics. When assessing pain, it is extremely important to note that many of the trials in adults were unblinded. Most blinded trials reported similar results to the unblinded ones. In conclusion, one may still doubt, on justified grounds, whether pain is truly less after LA, and, if so, whether this is of clinical relevance (Eypasch 1996).

Hospital stay was shortened by almost one day after LA. However, the study results were highly heterogeneous. It is obvious that the length of hospital stay depends on further circumstances, such as the health care system. The finding was similar to the finding of return to normal activity that allowed only some very cautious conclusions because of heterogeneity, although there was a uniform tendency to quicker recovery after LA. Only two studies reported results on quality of life in adults two weeks, six weeks, and six months after surgery. The Gastro-intestinal Quality of Life Index and SF-36 questionnaire are validated scales and widely used in trials. No study reported data on quality of life in children. In principle, both scores may be applied to children. However, children are not adults and there is possibly a more appropriate instrument available.

The effect of blinding was of critical importance in the evaluation of laparoscopic cholecystectomy (Majeed 1996). It may well be possible that participants who were randomised to LA and their caregivers had high expectations since LA is the more 'modern' 
procedure. Therefore, since nearly all results might have been influenced by such effects (Neugebauer 1991), these results should all be confirmed by blinded studies.

It is worth noting that the current review was restricted to standard multi-trocar LA. During the last years, technological developments in instrument design have allowed surgeons to reduce the invasiveness of appendectomy even further. Single incision laparoscopic surgery (SILS) and natural orifice transluminal endoscopic surgery (NOTES) are novel techniques with the potential to minimize the morbidity of surgery. Reviews have already compared single incision versus multi-incision appendectomy (Aly 2016; Rehman 2011). In this sense, moving from OA to LA appears just one step on a long ladder.

\section{Overall completeness and applicability of evidence}

We included all randomised trials meeting the inclusion criteria so that this review reflects the best currently available evidence. The range of countries from which the included studies originated is broad. The same holds true for the patients' characteristics. Furthermore, different levels of surgical proficiency were represented in the studies. Still, several results of the present review were homogeneous among the studies, which indicates that findings are apparently robust to various external factors. Therefore, the results of this review are widely applicable.

Our analyses included some additional studies that were either published only as abstracts, book chapters, or even master theses. As we found some such studies, we cannot entirely rule out that we have missed others, but it can be reasonably assumed that such studies are likely to be very small in size and therefore would have little impact on our results. Furthermore, the funnel plot analysis showed no signs of asymmetry.

\section{Quality of the evidence}

The quality of the evidence varied from very low to moderate (Summary of findings for the main comparison; Summary of findings 2; Summary of findings 3; Summary of findings 4). The main reasons for downgrading were the unclear risk of performance bias and outcome reporting bias. We also downgraded further for inconsistency and for imprecision because of unexplained heterogeneity and large confidence intervals, respectively.

\section{Potential biases in the review process}

The comprehensive systematic search for published and unpublished randomised trials is the strength of our review. We included trials irrespective of language and all important process steps were performed independently by two review authors. We have described all registered ongoing studies. Additionally, we contacted authors for further data. In total, we reduced the possibility of bias by our methodological approach. We are aware of five abstracts that have not been published as full text. Therefore, we cannot exclude a potential publication bias.

However, the included studies are an important source of bias because most participants, personnel, and outcome assessors were not blinded. Additionally, because of missing study protocols and study registries, we cannot assess the prevalence of outcome reporting bias. These biases are an inherent part of this review.
Regarding adverse events, we analysed the wound infections and intra-abdominal abscesses that were reported in most studies. Other adverse events such as iatrogenic bowel injuries were not stated systematically by the authors. Therefore, the presentation of rare adverse events may be incomplete.

\section{Agreements and disagreements with other studies or reviews}

In 2015, an overview of systematic reviews comparing LA with OA in patients with suspected appendicitis was published (Jaschinski 2015). The results of nine systematic reviews (Bennett 2007; Golub 1998; Li 2010; Liu 2010; Meynaud-Kraemer 1999; Ohtani 2012; Temple 1999; Wei 2011), including the version of this review published in 2010 (Sauerland 2010), showed discordant findings concerning the magnitude of the effect but not the direction of the effect.

Three reviews reported a reduction of pain on postoperative day one in favour of LA compared with OA (Bennett 2007; Li 2010; Sauerland 2010), but the effect sizes (ranging from - 0.8 to -0.7 points on a 10 point-VAS) were significant in only two of the reviews ( $\mathrm{Li}$ 2010; Sauerland 2010). In addition, these findings were affected by strong heterogeneity. The incidence of wound infections analysed by all reviews was significantly less for LA and the OR ranged from 0.3 to 0.52 with low heterogeneity across the randomised trials. In total, six reviews estimated the OR for intra-abdominal abscesses, ranging from 1.56 to 2.29 . Three reviews detected no significant difference between LA and OA (Golub 1998; Temple 1999; Wei 2011) but three others showed significantly higher rates of intraabdominal abscesses for LA (Bennett 2007; Li 2010; Sauerland 2010). Systematic reviews published after 2015 (Dai 2017; Ukai 2016) did not disagree with these results.

\section{AUTHORS' CONCLUSIONS}

\section{Implications for practice}

Except for a lower rate of intra-abdominal abscesses after $O A$ in adults, LA showed advantages over OA in postsurgical pain, wound infections, length of hospital stay, and time until return to normal activity in adults and children. Two studies reported better quality of life scores in adults. No study reported this outcome in children. However, the quality of evidence ranged from very low to moderate and some of the clinical effects of LA were small and of limited clinical relevance.

Despite the advantages of laparoscopic surgery for suspected appendicitis, one must not forget that most of the laparoscopic surgeons involved in these trials were well trained experts in laparoscopy. Therefore, surgical expertise with laparoscopic techniques is a basic prerequisite before surgeons can justifiably expect clinical benefits from LA. Nevertheless, formal laparoscopic training is nowadays offered in most residency training programmes. The fact that the included trials were conducted in highly but also less developed countries shows that the current results are applicable to most countries of the world.

\section{Implications for research}

Since a large number of studies was available for most comparisons in this review, future research should either use better study designs or focus on more specific aspects of the issue, such as specific outcomes. Only two studies reported quality of life in adults 
and no study reported this outcome in children. Additionally, we want to encourage other researchers to publish their results as full publications since we are aware of five studies only available as abstracts (Barth 1999; Esposito 1997; Hoff 1995; Loh 1992; Rohr 1994).

Furthermore, only eight authors of the included studies reported a sample size calculation. One may conclude that the other trials were not appropriately powered. This assumption is supported by the missing specification of the primary outcome in these studies. For future studies, statistical consulting is essential to ensure the usefulness of the results.

Most studies included in this review had a follow-up between a few days of hospital stay and a month. A much longer followup period of present and future studies is necessary to find out whether the lower rate of adhesions first found by DeWilde (DeWilde 1991) translates into a clinically relevant reduction of adhesion-related complications, such as ileus. Observational data have indicated that LA, as opposed to OA, might reduce adhesive bowel obstruction in the long term (Markar 2014).

The assessment of pain (and also other outcomes) should be done in a blinded manner in future studies. Furthermore, standard regimens of pain therapy should be employed in both study arms. Researchers should decide whether to measure pain and standardise pain therapy, or to measure analgesic consumption and keep participants below a standard level of pain. Studies where both analgesics and pain intensity vary are difficult to interpret when the results for both are in the opposite direction.
Future studies with low risk of bias should investigate, in particular, the quality of life in children.

\section{ACKNOWLEDGEMENTS}

We would like to thank all trialists who provided us with additional data, preliminary manuscripts, details of trial design, and references to further studies. The names of these trialists are (in alphabetical order): Prof. Akhtar (Karachi, Pakistan), Dr. S. Askarpour (Ahvaz, Iran), Dr. D. Bliss (Los Angeles, USA), Dr. R.L. DeWilde (Oldenburg, Germany), Dr. M. Gonenc (Istanbul, Turkey), Dr. M. Hasbahceci (Istanbul, Turkey), Prof. S.A. Hay (Cairo, Egypt), Dr. M.T. Huang (Taipei, Taiwan), Prof. Dr. W.A. Jan (Peshawar, Pakistan), Dr. K. Karadayi (Sivas, Turkey), Dr. H. Kokki (Kuopio, Finland), Dr. A. Koluh (Nova Bila, Bosnia and Herzegovina), Dr. M. Lavonius (Turku, Finland), Capt. Paul Lucha (Portsmouth/VA, USA), Dr. E. Macarulla (Barcelona, Spain), Dr. M. Milewczyk (Wejherowo, Poland), Dr. G. Navarra (Ferrara, Italy), Dr. K. Paya (Vienna, Austria), Dr. A. Rashid (New Delhi, India), Prof. M.G. Sarr (Rochester/MN, USA), Dr. M. Schietroma (L'Aquila, Italy), Dr. U. Settmacher (Berlin, Germany), Prof. A. Sezeur (Paris, France), Dr. A.Sozutek (Mersin, Turkey), Dr. R. Stare (Varazdin, Croatia), Dr. G. Tzovaras (Larissa, Greece), Dr. F. Vallribera Valls (Barcelona, Spain), Dr. P. Wara (Aarhus, Denmark), Prof. B.L. Warren (Tygerberg, South Africa), Dr. I. Witten and Prof. H.F. Weiser (Rotenburg/Wümme, Germany), and Dr. C.K. Yeung (Hongkong, Hongkong).

We thank Managing Editor Henning Keinke Andersen and the CCCG editors for providing relevant suggestions that improved the review. Additionally, we thank Sys Johnsen and Josephine Lyngh Steenberg for running the searches in Ovid. 


\section{R E F E R E N C E S}

\section{References to studies included in this review}

Aktimur 2016 \{published data only\}

Aktimur R, Gokakin AK, Deveci K, Atabey M, Topcu O. Oxidative stress markers in laparoscopic vs. open appendectomy for acute appendicitis: a double-blind randomized study. Journal of Minimal Access Surgery 2016;12(2):143-7. [PUBMED: 27073307]

Al-Mulhim 2002 \{published data only\}

* Al-Mulhim AS, Al-Mulhim FM, Al-Suwaiygh AA, Al-Masaud NA. Laparoscopic versus open appendectomy in females with a clinical diagnosis of appendicitis. Saudi Medical Journal 2002;23(11):1339-42. [PUBMED: 12506292]

Attwood 1992 \{published data only\}

* Attwood SE, Hill AD, Murphy PG, Thornton J, Stephens RB. A prospective randomized trial of laparoscopic versus open appendectomy. Surgery 1992;112(3):497-501. [PUBMED: 1387739]

Barth 1999 \{published data only\}

* Meynaud-Kraemer L, Colin C, Vergnon P, Barth X. Wound infection in open versus laparoscopic appendectomy. International Journal of Technology Assessment in Health Care 1999;15(2):380-91. [PUBMED: 10507196]

Bartin 2016 \{published data only\}

Bartin MK, Kemik O, Caparlar MA, Bostanci MT, Oner MO. Evaluation of the open and laparoscopic appendectomy operations with respect to their effect on serum il-6 levels. Ulusal Travma ve Acil Cerrahi Dergisi 2016;22(5):466-70. [EMBASE: 612110477]

\section{Bauwens 1999 \{published data only\}}

* Bauwens K, Schwenk W, Böhm B, Hasart O, Neudecker J, Müller JM. Convalescence and time to return to work after laparoscopic and open appendectomy; a prospective randomized study [Rekonvaleszenz und Arbeitsunfähigkeitsdauer nach laparoskopischer und konventioneller Appendekotmie. Eine prospektiv-randomisierte Studie]. Chirurg 1998;69:541-5. [PUBMED: 9653559]

Hasart O. Rekonvaleszenz und Arbeitsunfähigkeitsdauer nach laparoskopischer undkonventioneller Appendektomie: eine prospektiv-randomisierte Studie [Masters thesis]. Berlin (Germany): Humboldt University of Berlin, 2000.

Schwenk W, Bauwens K, Neudecker J, Böhm B. Duration of reconvalecense and inability to work after laparoscopic and conventional appendectomy - a prospective randomised study [Rekonvaleszenz und Arbeitsunfähigkeitsdauer nach laparoskopischer und konventioneller Appendektomie - eine prospektiv-randomisierte Studie]. Zentralblatt für Chirurgie 1998;123:424-5.

\section{Bliss 2014 \{published and unpublished data\}}

Bliss D, Mckee J, Cho D, Krishnaswami S, Zallen G, Harrison M, et al. Discordance of the pediatric surgeon's intraoperative assessment of pediatric appendicitis with the pathologists report. Journal of Pediatric Surgery 2010;45(7):1398-403. [PUBMED: 20638515]

* Bliss D, Mckee J, Cho D, Krishnaswami S, Zallen G, Silen M, et. al. Results of a prospective, randomized, controlled, singleblinded trial of laparoscopic versus open appendectomy in children. Journal of the American College of Surgeons (in press).

\section{Bolla 2008 \{published data only\}}

Bolla G, Tuzzato G. Post-appendectomy immunologic competence in pediatric age. The results do not always match expectations. Laparoscopy versus laparotomy. Pediatria Medica e Chirurgica 2008;30(2):89-93. [PUBMED: 18581961]

\section{Bruwer 2000 \{published data only\}}

Bruwer F, Coetzer M, Warren BL. Early results of a randomized controled study of laparoscopic vs open surgical exploration in pre-menopausal women with suspected acute appendicitis. Surgical Endoscopy. 2000; Vol. 14, issue Suppl:S110.

* Bruwer F, Coetzer M, Warren BL. Laparoscopic versus open surgical exploration in premenopausal women with suspected acute appendicitis. South African Journal of Surgery 2003;41(4):82-5. [PUBMED: 14768141]

Cipe 2014 \{published and unpublished data\}

Cipe G, Hasbahceci M, Bozkurt S, Kadioglu H, Coskun H, Karatepe O, et. al. Laparoscopic versus open appendectomy: where are we now?. Chirurgia (Bucur) 2014;109(4):518-22. [PUBMED: 25149616]

Cox 1996 \{published data only\}

* Cox MR, McCall JL, Toouli J, Padbury RT Wilson TG, Wattchow DA, et. al. Prospective randomized comparison of open versus laparoscopic appendectomy in men. World Journal of Surgery 1996;20(3):263-6. [PUBMED: 8661828]

DeWilde 1991 \{published data only (unpublished sought but not used)\}

* De Wilde RL. Goodbye to late bowel obstruction after appendicectomy. Lancet 1991;338(8773):1012. [PUBMED: 1681313]

Eichen 1994 \{published and unpublished data\}

* Eichen R, Heuser H, Nitschke B. Laparoscopic or conventional appendectomy:a prospective study [Prospektive Studie: laparoskopische Appendektomie vs. konventionelle Appendektomie]. Langenbecks Archiv fur Chirurgie 1994;111:223-5. [DOI: 10.1007/978-3-642-79242-7_54]

Frazee 1994 \{published data only\}

* Frazee RC, Roberts JW, Symmonds RE, Snyder SK, Hendricks JC, Smith RW, et. al. A prospective randomized trial comparing open versus laparoscopic appendectomy. Annals of Surgery 1994;219(6):725-31. [PUBMED: 8203983]

Goudar 2011 \{published data only\}

* Goudar BV, Telkar S, Lamani YP, Shirbur SN, Shailesh ME. Laparoscopic versus open appendectomy: a comparison of 
primary outcome studies from Southern India. Journal of Clinical and Diagnostic Research 2011;5(8):1606-9. [EMBASE: 2012053242]

\section{Gundavda 2012 \{published data only\}}

Gundavda MK, Bhandarwar AH. Comparative study of laparoscopic versus open appendicectomy. Indian Journal of Medical Sciences 2012;66(5-6):99-115. [PUBMED: 23806983]

\section{Hansen 1996 \{published data only\}}

* Hansen JB, Smithers BM, Schache D, Wall DR, Miller BJ, Menzies BL. Laparoscopic versus open appendectomy: prospective randomized trial. World Journal of Surgery 1996;20(1):17-20. [PUBMED: 8588406]

Smithers BM, Hansen J, Schache D, Wall D. Prospective randomized trial of laparoscopic versus open appendicectomy for acute appendicitis. Surgical Endoscopy. 1994; Vol. 8:565.

\section{Hart 1996 \{published data only\}}

* Hart R, Rajgopal C, Plewes A, Sweeney J, Davies W, Gray D, et al. Laparoscopic versus open appendectomy: a prospective randomized trial of 81 patients. Canadian Journal of Surgery 1996;39(6):457-62. [PUBMED: 8956810]

\section{Hebebrand 1994 \{published and unpublished data\}}

Eypasch E, Menningen R, Paul A, Troidl H. Diagnostic and therapeutic laparoscopy for acute abdominal disorders [Die Bedeutung der Laparoskopie bei der Diagnostik und Therapie des akuten Abdomens]. Zentralblatt für Chirurgie 1993;118(12):726-32. [PUBMED: 8147145]

* Hebebrand D, Troidl H, Spangenberger W, Neugebauer E, Schwalm T, Günther MW. Laparoscopic or classical appendectomy? A prospective randomized study [Laparoskopische oder klassische Appendektomie? Eine prospektiv randomisierte Studie]. Der Chirurg 1994;65(2):112-20. [PUBMED: 8162812]

Neugebauer E, Eypasch E, Hebebrand D, Spangenberger W, Troidl $\mathrm{H}$. [Laparoscopic versus conventional appendectomy - a prospective randomized study]. Proceedings of the 1st European Congress of the European Association for Endoscopic Surgery. 1993:371.

Schwalm T. Laparoskopische oder konventionelle Appendektomie - eine prospektive, randomisierte Therapiestudie unter besonderer Berücksichtigung der relevanten Zielkriterien Sicherheit und Patientenkomfort [Masters thesis]. Cologne (Germany): University of Cologne, 1995.

\section{Heikkinen 1998 \{published data only\}}

* Heikkinen TJ, Haukipuro K, Hulkko A. Cost-effective appendectomy. Open or laparoscopic? A prospective randomized study. Surgical Endoscopy 1998;12(10):1204-8. [PUBMED: 9745057]

\section{Hellberg 1999 \{published data only\}}

Enochsson L, Hellberg A, Rudberg C, Fenyö G, Gudbjartson T, Kullman E, et al. Laparoscopic vs. open appendectomy in overweight patients. Surgical Endoscopy 2001;15(4):387-92 [PUBMED: 11395821]

Hellberg A, Rudberg C, Enochsson L, Gudbjartson T, Wenner J, Kullman E, et al. Conversion from laparoscopic to open appendicectomy: a possible drawback of the laparoscopic technique?. European Journal of Surgery 2001;167(3):209-13. [PUBMED: 11316407]

* Hellberg A, Rudberg C, Kullman E, Enochsson L, Fenyö G, Graffner $\mathrm{H}$, et al. Prospective randomized multicentre study of laparoscopic versus open appendicectomy. British Journal of Surgery 1999;86(1):48-53. [PUBMED: 10027359]

\section{Helmy 2001 \{published data only\}}

* Helmy MA. A comparative study between laparoscopic versus open appendicectomy in men. Journal of the Egyptian Society of Parasitology 2001;31(2):555-62.. [PUBMED: 11478454]

\section{Henle 1996 \{published data only\}}

* Henle KP, Beller S, Rechner J, Zerz A, Klingler A. Laparoscopic versus open appendectomy: a prospecitve randomized trial [Laparoskopische versus konventionelle Appendektomie: eine prospektive, randomisierte Studie]. Der Chirurg 1996;67(5):526-30. [PUBMED: 8777883]

Klingler A, Henle KP, Beller S, Rechner J, Zerz A, Wetscher GJ, et. al. Laparoscopic appendectomy does not change the incidence of postoperative infectious complications. American Journal of Surgery 1998;175(3):232-35. [PUBMED: 9560127]

Szinicz G, Henle KP, Beller S, Rechner J, Zerz A, Klingler A. Laparoscopic appendectomy: Pro [Laparoskopische Appendektomie: Pro]. In: Boeckl O, Waclawiczek HW editor(s). Standards in der Chirurgie. Munich (Germany): Zuckschwerdt, 1995:312-9.

\section{Huang 2001 \{published and unpublished data\}}

* Huang MT, Wei PL, Wu CC, Lai IR, Chen RJ, Lee WJ. Needlescopic, laparoscopic, and open appendectomy: a comparative study. Surgical Laparoscopy, Endoscopy \& Percutaneous Techniques 2001;11(5):306-12. [PUBMED: 11668227]

\section{Ignacio 2003 \{published data only\}}

* Ignacio RC, Burke R, Spencer D, Bissell C, Dorsainvil C, Lucha PA. Laparoscopic vs open appendectomy. What is the real difference? Results of a prospective randomized double-blinded trial. Surgical Endoscopy 2004;18(2):334-7. [PUBMED: 14691696]

Ignacio RC, Lucha PA Jr, Burke R, Newman J, Shepps C, Spencer D, et al. Laparoscopic versus open appendectomy What is the real difference? Results of a prospective randomized double-blinded trial. Surgical Endoscopy 2003;17:S239.

\section{Jadallah 1994 \{published data only\}}

Jadallah FA, Abdul-Ghani AA, Tibblin S. Diagnostic laparoscopy reduces unneccessary appendicectomy in fertile women. European Journal of Surgery 1994;160(1):41-5. [PUBMED: 8186313] 
Kaiser 2006 \{published data only\}

* Kaiser M. Laparoskopische und konventionelle Appendektomie im Vergleich unter besonderer Berücksichtigung immunologischer Parameter - eine prospektive Studie [Master thesis]. Magdeburg (Germany): University of Magdeburg, 1996.

\section{Kald 1999 \{published data only\}}

* Kald A, Kullman E, Anderberg B, Wirén M, Carlsson P, Ringqvist I, et al. Cost-minimisation analysis of laparoscopic and open appendicectomy. European Journal of Surgery 1999;165(6):579-82. [PUBMED: 10433143]

\section{Kaplan 2009 \{published data only\}}

* Kaplan M, Salman B, Yilmaz TU, Oguz M. A quality of life comparison of laparoscopic and open approaches in acute appendicitis: a randomised prospective study. Acta Chirurgica Belgica 2009;109(3):356-63. [PUBMED: 19943593]

\section{Karadayi 2003 \{published and unpublished data\}}

* Karadayi K, Turan M, Canbay E, Topcu O, Sen M. Laparoscopic versus open appendectomy: analysis of systemic acutephase responses in a prospective randomized study. Chirurgia Gastroenterologica 2003;19(4):396-400.

\section{Kargar 2011 \{published data only\}}

* Kargar S, Mirshamsi MH, Zare M, Arefanian S, Yazdi ES, Aref A. Laparoscopic versus open appendectopmy; which method to choose? A prospective randomized comparison. Acta Medica Iranica 2011;49(6):352-6. [PUBMED: 21874637]

\section{Katkhouda 2005 \{published data only\}}

Clarke T, Katkhouda N, Mason RJ, Cheng BC, Olasky J, Sohn HJ, et al. Laparoscopic versus open appendectomy for the obese patient: a subset analysis from a prospective, randomized, double-blind study. Surgical Endoscopy 2011;25(4):1276-80. [PUBMED: 21046164]

* Katkhouda N, Mason RJ, Towfigh S, Gevorgyan A, Essani R. Laparoscopic versus open appendectomy: a prospective randomized double-blind study. Annals of Surgery 2005;242(3):439-50. [PUBMED: 16135930]

\section{Kazemier 1997 \{published data only\}}

Kazemier G, de Zeeuw GR, Bonjer HJ, Lange JF. Laparoscopic vs open appendectomy. A randomized clinical trial. Surgical Endoscopy. 1996; Vol. 10:550.

Kazemier G, de Zeeuw GR, Bonjer HJ, Lange JF. Laparoscopic vs open appendectomy. A randomized clinical trial. Surgical Endoscopy. 1995; Vol. 9:213.

Kazemier G, de Zeeuw GR, Bonjer HJ, Lange JF. Laparoscopic vs open appendectomy. A randomized clinical trial. Surgical Endoscopy. 1995; Vol. 9:606.

* Kazemier G, de Zeeuw GR, Lange JF, Hop WCJ, Bonjer HJ. Laparoscopic vs open appendectomy. A randomized clinical trial. Surgical Endoscopy 1997;11(4):336-40. [PUBMED: 9094272]

de Zeeuw GR, Kazemier G, Hop WCJ, Lange JF, Bonjer HJ. Laparoscopic treatment of acute appendicitis: less pain and wound infection but longer operating time; a randomized trial [Laparoscopische behandling van appendicitis acuta: minder pijn en wondinfecties, maar langere operatietijd; een gerandomiseerd onderzoek]. Nederlands Tijdschrift Geneeskunde 1998;142(4):459-63. [EMBASE: 1998081176]

\section{Kehagias 2009 \{published and unpublished data\}}

Kehagias I, Karamanakos S, Panagiotopoulos S, Vagenas K, Kalfarentzos F. Laparoscopic versus open appendectomy for complicated disease - a prospective randomized trial. Surgical Endoscopy. 2009; Vol. 23, issue Suppl:13.

\section{Khalil 2011 \{published data only\}}

Khalil J, Muqim R, Rafique M, Khan M. Laparoscopic versus open appendectomy: a comparison of primary outcome measures. Saudi Journal of Gastroenterology: Official Journal of the Saudi Gastroenterology Association 2011;17(4):236-40. [PUBMED: 21727728]

\section{Kocatas 2013 \{published and unpublished data\}}

Kocatas A, Gönenc M, Bozkurt MA, Karabulut M, Gemici E, Alis H. Comparison of open and laparoscopic appendectomy in uncomplicated appendicitis: a prospective randomized clinical trial. Ulusal Travma ve Acil Cerrahi Dergisi 2013;19(3):200-4. [PUBMED: 23720105]

Kouhia 2010 \{published data only\}

* Kouhia ST, Heiskanen JT, Huttunen R, Ahtola HI, Kiviniemi VV, Hakala T. Long-term follow-up of a randomized clinical trial of open versus laparoscopic appendicectomy. British Journal of Surgery 2010;97(9):1395-400. [PUBMED: 20632312]

Kum 1993a \{published data only (unpublished sought but not used)\}

${ }^{*}$ Kum CK, Ngoi SS, Goh PM, Tekant Y, Isaac JR. Randomized controlled trial comparing laparoscopic and open appendicectomy. British Journal of Surgery 1993;80(12):1599-600. [PUBMED: 8298936]

\section{Laine 1997 \{published data only\}}

* Laine S, Rantala A, Gullichsen R, Ovaska J. Laparoscopic appendectomy-is it worthwhile? A prospective, randomized study in young women. Surgical Endoscopy 1997;11(2):95-7. [PUBMED: 9069134]

Laine S, Rantala A, Gullichsen R, Ovaska J. Laparoscopic versus conventional appendectomy: a prospective, randomized study in young women. Surgical Endoscopy. 1996; Vol. 10:243.

\section{Larsson 2001 \{published data only\}}

* Larsson PG, Henriksson G, Olsson M, Boris J, Ströberg P, Tronstad SE, et al. Laparoscopy reduces unnecessary appendicectomies and improves diagnosis in fertile women. A randomized study. Surgical Endoscopy 2001;15(2):200-2. [MEDLINE: 11285968]

\section{Lavonius 2001 \{published and unpublished data\}}

* Lavonius MI, Liesjärvi S, Ovaska J, Pajulo O, Ristkari S, Alanen M. Laparoscopic versus open appendectomy in children: a prospective randomised study. European Journal of Pediatric Surgery 2001;11(4):235-38. [PUBMED: 11558012] 


\section{Lejus 1996 \{published data only\}}

Delile L. Etude comparative de l'analgesie post-operatoire apres appendectomie par incision de MacBurney ou par voie coelioscopique chez l'enfant [Masters thesis]. Nantes (France): University of Nantes, 1994.

Heloury Y, Lejus C, Delile L, Plattner V, Baron M, Souron R. Postoperative pain after appendicectomy: Laparoscopic versus MacBurney. Surgical Endoscopy. 1994; Vol. 8:958.

* Lejus C, Delile L, Plattner V, Baron M, Guillou S, Heloury Y, et al. Randomized, single-blinded trial of laparoscopic versus open appendectomy in children: effects on postoperative analgesia. Anesthesiology 1996;84(4):801-6. [PUBMED: 8638833]

\section{Lintula 2004 \{published and unpublished data\}}

Lintula H, Kokki H, Vanamo K. Single-blind randomized clinical trial of laparoscopic versus open appendicectomy in children. British Journal of Surgery 2001;88(4):510-4. [PUBMED: 11298617]

Lintula H, Kokki H, Vanamo K, Antila P, Eskelinen M. Laparoscopy in children with complicated appendicitis. Journal of Pediatric Surgery 2002;37(9):1317-20.

* Lintula H, Kokki H, Vanamo K, Valtonen H, Mattila M, Eskelinen M. The costs and effects of laparoscopic appendectomy in children. JAMA Pediatrics 2004;158(1):34-7. [PUBMED: 14706955]

\section{Little 2002 \{published data only\}}

* Little DC, Custer MD, May BH, Blalock SE, Cooney DR. Laparoscopic appendectomy: an unnecessary and expensive procedure in children?. Journal of Pediatric Surgery 2002;37(3):310-17. [PUBMED: 11877640]

\section{Long 2000 \{unpublished data only\}}

Bannon MP, Zietlow SP, Harmsen WS, Sarr MG, Smith CD, Ilstrup DM, et. al. Prospective randomized comparison of laparoscopic and open appendectomy. Gastroenterology. 1997; Vol. 112.

* Long KH, Bannon MP, Zietlow SP, Helgeson ER, Harmsen WS, Smith $C D$, et. al. A prospective randomized comparison of laparoscopic appendectomy with open appendectomy: clinical and economic analysis. Surgery 2001;129(4):390-400. [PUBMED: 11283528]

\section{Macarulla 1995 \{published data only (unpublished sought but not} used)\}

Macarulla E, Hassan H, Abad JM, Vallet J, Claveria R, Besora P, et al. Laparoscopic vs open appendectomy for suspected appendicitis. British Journal of Surgery. 1995; Vol. 82, issue Suppl:6-7.

* Macarulla E, Vallet J, Abad JM, Hussein H, Fernandez E, Nieto B. Laparoscopic versus open appendectomy: a prospective randomized trial. Surgical Laparoscopy \& Endoscopy 1997;7(4):335-9. [PUBMED: 9282768]
Mahmood 2016 \{published data only\}

Mahmood MM, Shahab A, Razzaq MA. Surgical site infection in open versus laparoscopic appendectomy. Pakistan Journal of Medical and Health Sciences 2016;10(3):1076-8. [EMBASE: 614365275]

\section{Martin 1995 \{published data only\}}

* Martin LC, Puente I, Sosa JL, Bassin A, Breslaw R, McKenney MG, et al. Open versus laparoscopic appendectomy. A prospective randomized comparison. Annals of Surgery 1995;222(3):256-62. [PUBMED: 7677456]

Minné 1997 \{published data only\}

Jones B, Ratzer E, Clark J, Zeren F, Haun W. Does peer-reviewed publication change the habits of surgeons?. American Journal of Surgery 2000;180(6):566-9. [PUBMED: 11182419]

* Minné L, Varner D, Burnell A, Ratzer E, Clark J, Haun W. Laparoscopic vs open appendectomy. Prospective randomized study of outcomes. Archives of Surgery 1997;132(7):708-12. [PUBMED: 9230853]

\section{Moberg 2005 \{published data only\}}

Moberg AC, Berndsen F, Palmquist I, Petersson U, Resch T, Montgomery A. Randomized clinical trial of laparoscopic versus open appendicectomy for confirmed appendicitis. British Journal of Surgery 2005;92(3):298-304. [PUBMED: 15609378]

Moirangthem 2008 \{published data only\}

Moirangthem GS, Arunkumar CH, Marak AB, Lokendra K, Singh LD. A comparative study between laparoscopic versus open appendicectomy. Journal of Management and Science 2008;22(2):58-62. [EMBASE: 2008368558]

\section{Mutter 1996 \{published data only\}}

* Mutter D, Vix M, Bui A, Evrard S, Tassetti V, Breton JF, et al. Laparoscopy not recommended for routine appendectomy in men: results of a prospective randomized study. Surgery 1996;120(1):71-4. [PUBMED: 8693426]

\section{Navarra 2000 \{published and unpublished data\}}

* Navarra G, Ascanelli S, Turini A, Carcoforo P, Tonini G, Pozza E. Laparoscopic appendectomy versus open appendectomy in suspected acute appendicitis in female patients [Appendicectomia laparoscopica versus appendicectomia aperta nel sospetto di appendicite acuta in pazienti di sesso femminile]. Annali Italiani di Chirurgia 2002;73(1):59-63. [PUBMED: 12148423]

Navarra G, Ascanelli S, Turini A, Tonini G, Pozza E. Laparoscopic versus open appendectomy in females with pain in right iliac fossa. Surgical Endoscopy. 2000; Vol. 14, issue Suppl:S128.

\section{Nordentoft 2000 \{published data only\}}

* Nordentoft T, Bringstrup FA, Bremmelgaard A, Stage JG. Effect of laparoscopy on bacteremia in acute appendicitis: a randomized controlled study. Surgical Laparoscopy, Endoscopy \& Percutaneous Techniques 2000;10(5):302-4. [PUBMED: $11083213]$ 
Olsen 1993 \{published data only\}

Olsen JB, Myren CJ, Haahr PE. Randomized trial on the value of diagnostic laparoscopy before appendectomy. Ugeskrift for Laeger 1995;157(5):584-85. [PUBMED: 7638913]

* Olsen JB, Myrén CJ, Haahr PE. Randomized study of the value of laparoscopy before appendicectomy. British Journal of Surgery 1993;80(7):922-3. [PUBMED: 8369940]

\section{Ortega 1996 \{published data only\}}

* Ortega AE, Hunter JG, Peters JH, Swanstrom LL, Schirmer B. A prospective, randomized comparison of laparoscopic appendectomy with open appendectomy. American Journal of Surgery 1995;169(2):208-13. [PUBMED: 7840381]

\section{Özmen 1999 \{published data only\}}

* Özmen MM, Zülfikaroglu B, Tanik A, Kale IT. Laparoscopic versus open appendectomy: prospective randomized trial. Surgical Laparoscopy, Endoscopy \& Percutaneous Techniques 1999;9(3):187-9. [PUBMED: 10803997]

\section{Pedersen 2001 \{published data only\}}

* Pedersen AG, Petersen OB, Wara P, Rønning H, Qvist N, Laurberg S. Randomised controlled trial of laparoscopic versus open appendicectomy. British Journal of Surgery 2001;88(2):200-5. [PUBMED: 11167866]

\section{Perner 1999 \{published data only\}}

* Perner A, Bugge K, Lyng KM, Schulze S, Kristensen PA, Bendtsen A. Changes in plasma potassium concentration during carbon dioxide pneumoperitoneum. British Journal of Anaesthesia 1999;82(1):137-9. [PUBMED: 10325852]

\section{Pozo 1996 \{published data only\}}

Pozo JC, Martinez S, Negri C, Rachadell JJ, Belloso R. Comparative controlled study: laparoscopic vs. conventional appendectomies in a teaching program general surgery [Estudio prospectivo y controlado: comparando la apendicectomía laparoscópica y la apendicectomía convencional en un programa de entrenamiento de postgrado en cirugía general]. Revista de la Sociedad Venezolana de Gastroenterologia 1996;50(4):225-30. [LILACS: 261607]

\section{Reiertsen 1997 \{published data only\}}

* Reiertsen O, Larsen S, Trondsen E, Edwin B, Færden AE, Rosseland AR. Randomized controlled trial with sequential design of laparoscopic versus conventional appendicectomy. British Journal of Surgery 1997;84(6):842-7. [PUBMED: 9189105]

Ricca 2007 \{published and unpublished data\}

* Ricca R, Schneider JJ, Brar H, Lucha PA. Laparoscopic appendectomy in patients with a body mass index of 25 or greater: results of a double blind, prospective, randomized trial. Journal of the Society of Laparoendoscopic Surgeons 2007;11(1):54-8. [PUBMED: 17663093]

\section{Saha 2010 \{published data only\}}

* Saha N, Saha DK, Rahman MA, Islam MK, Aziz MA. Comparison of post operative morbidity between laparoscopic and open appendectomy in children. Mymensingh Medical Journal 2010;19(3):348-52. [PUBMED: 20639825]
Schietroma 2012 \{published and unpublished data\}

* Schietroma M, Piccione F, Carlei F, Clementi M, Bianchi Z, de Vita F, et al. Peritonitis from perforated appendicitis: stress response after laparoscopic or open treatment. The American Surgeon 2012;78(5):582-90. [PUBMED: 22546132]

\section{Schippers 1997 \{published data only\}}

Schippers E. Appendicectomy - laparoscopic vs open [Appendektomie - laparoskopisch vs konventionell]. Langenbecks Archiv fur Chirurgie 1993;110:142-5.

Schippers E, Pier A, May P, Schumpelick V. Laparoscopic vs open appendectomy - a comparison of postoperative pain and respiratory function. Surgical Endoscopy. 1994; Vol. 8:561.

\section{Settmacher 1995 \{published data only\}}

* Settmacher U, Manger T, Liebenthal C, Neuhaus K, Volk HD. Immunological modifications after open and laparoscopic surgery. Minimal Invasive Chirurgie 1995;4(2):95-102. [CENTRAL: CN-00384222]

\section{Sezeur 1997 \{published data only\}}

* Sezeur A, Bure-Rossier AM, Rio D, Savigny B, Tricot C, Martel P, et. al. Does laparoscopy increase the bacteriological risk of appendectomy? Results of a randomized prospective study [La cœlioscopie augmente-t-elle le risque bactériologique de l'appendicectomie? Résultats d'une étude prospective randomisée]. Annales de Chirurgie 1997;51(3):243-7. [PUBMED: 9297886]

\section{Simon 2009 \{published data only\}}

Simon P, Burkhardt U, Sack U, Kaisers UX, Muensterer OJ. Inflammatory response is no different in children randomized to laparoscopic or open appendectomy. Journal of Laparoendoscopic \& Advanced Surgical Techniques. Part A. 2009; Vol. 19, issue Suppl:S71-6.

\section{Singh 2017 \{published data only\}}

Singh VK, Nishant K, Kharga B, Kalita AK, Bhutia P, Jain J. Randomized controlled trial comparing open, conventional, and single port laparoscopic appendectomy. Journal of Clinical and Diagnostic Research 2017;11(10):5-10. [EMBASE: 618835726]

\section{Sozutek 2013 \{published and unpublished data\}}

Sozutek A, Colak T, Dirlik M, Ocal K, Turkmenoglu O, Dag A. A prospective randomized comparison of single-port laparoscopic procedure with open and standard 3-port laparoscopic procedures in the treatment of acute appendicitis. Surgical Laparoscopy, Endoscopy \& Percutaneous Techniques 2013;23(1):74-8. [PUBMED: 23386157]

Stare 1998 \{published data only (unpublished sought but not used)\}

Stare R, Kocman I, Povšic Cevra Z. Results of a prospective randomised study of laparoscopic appendectomy in community hospital. Surgical Endoscopy. 1998; Vol. 12:573.

* Stare R, Kocman I, Povšic Cevra Z, Zgrebec Z, Kovacic D. Results of a prospective randomised study of laparoscopic appendectomy in community hospital. In: Montori A, Lirici MM, 
Montori J editor(s). 6th World Congress of Endoscopic Surgery. Rome (Italy): Monduzzi, 1998:441-5. [CENTRAL: CN-00776112]

Sun 1998 \{published data only\}

* Sun XL, Xu HB. Comparative study among open laparoscopic and video-assisted appendectomies. World Chinese Journal of Digestion 1998;6:710-1. [CENTRAL: CN-00284898]

\section{Taguchi 2015 \{published data only\}}

Taguchi Y, Komatsu S, Sakamoto E, Norimizu S, Shingu Y, Hasegawa H. Laparoscopic versus open surgery for complicated appendicitis in adults: a randomized controlled trial. Surgical Endoscopy 2015;30(5):1705-12. [PUBMED: 26275544]

Tate 1993a \{published data only\}

* Tate JJT, Dawson JW, Chung SCS, Lau WY, Li AKC. Laparoscopic versus open appendicectomy: prospective randomised trial. Lancet 1993;342:633-7. [PUBMED: 8103144]

\section{Thomson 2015 \{published data only\}}

Thomson JE, Kruger D, Jann-Kruger C, Kiss A, OmoshoroJones JA, Luvhengo T, et al. Laparoscopic versus open surgery for complicated appendicitis: a randomized controlled trial to prove safety. Surgical Endoscopy 2014;29(7):2027-32. [PUBMED: 25318368]

\section{Tzovaras 2010 \{published and unpublished data\}}

* Tzovaras G, Baloyiannis I, Kouritas V, Symeonidis D, Spyridakis M, Poultsidi A, et al. Laparoscopic versus open appendectomy in men: a prospective randomized trial. Surgical Endoscopy 2010;24(12):2987-92. [PUBMED: 20552369]

Tzovaras G, Liakou P, Baloyiannis I, Spyridakis M, Mantzos F, Tepetes K, et al. Laparoscopic appendectomy: differences between male and female patients with suspected acute appendicitis. World Journal of Surgery 2007;31(2):409-13. [PUBMED: 17219281]

Vallribera 2003 \{published and unpublished data\}

* Vallribera F, Sala J, Aguilar F, Espin E. Influence of laparoscopic surgery on perception of quality of life after appendectomy [Influencia de la cirugía laparoscópica en la percepción de la claidad de vida tras apendicectomia]. Cirugía Española 2003;73(2):88-94.

Van Dalen 2003 \{published data only\}

* Van Dalen R, Bagshaw PF, Dobbs BR, Robertson GM, Lynch AC, Frizelle FA. The utility of laparoscopy in the diagnosis of acute appendicitis in women of reproductive age: a prospective randomized controlled trial with long-term follow-up. Surgical Endoscopy 2003;17(8):1311-3. [PUBMED: 12739123]

\section{Wei 2010 \{published data only\}}

* Wei HB, Huang JL, Zheng ZH, Wei B, Zheng F, Qiu WS, et. al. Laparoscopic versus open appendectomy: a prospective randomized comparison. Surgical Endoscopy 2010;24(2):266-9. [PUBMED: 19517167]
Williams 1996 \{published data only\}

* Williams MD, Collins JN, Wright TF, Fenoglio ME. Laparoscopic versus open appendectomy. Southern Medical Journal 1996;89(7):668-74. [PUBMED: 8685751]

\section{Witten 1998 \{published data only\}}

Birth M, Witten I, Gadzepko E, Weiser HF. Laparoscopic vs open appendectomy for acute appendicitis: a prospective randomized trial. British Journal of Surgery. 1998; Vol. 85, issue Suppl:39. [CENTRAL: CN-00594479]

* Witten KI. Die chirurgische Behandlung der akuten Appendizitis: ein Methodenvergleich zwischen laparoskopischer und konventioneller Appendektomie im Rahmen einer prospektiv randomisierten Studie an zweihundert Patienten [Masters thesis]. Göttingen (Germany): University of Göttingen, 2001.

Yeung 1997 \{published data only (unpublished sought but not used)\}

* Yeung CK, Yip KF, Lee KH, Lau WY. The role of minimally invasive surgery in the management of acute appendicitis in children: a prospective randomized trial of laparoscopic vs conventional appendectomy. Asian Journal of Surgery. 1997; Vol. 20:S55.

Yin 1996 \{published data only\}

Yin WY, Lee MC, Cheng TJ, Chen HT, Huang SM. Laparoscopic versus open appendectomy: prospective randomized trial. Tzu Chi Medical Journal 1996;8(3):213-21. [Chinese Electronic Periodical Sevices: 25831$]$

Yu 2016 \{published data only\}

Yu G, Han A, Wang W. Comparison of laparoscopic appendectomy with open appendectomy in treating children with appendicitis. Pakistan Journal of Medical Sciences 2016;32(2):299-304. [PUBMED: 27182227]

Zhang 1998 \{published data only\}

* Zhang WF, Xu CX, Liu YH, Huang GJ. A randomized control study on the appendectomy of 103 cases with laparoscope or mini-inicision. Modern Journal of Physicians 1998;3(6):8-9.

\section{References to studies excluded from this review}

Ablassmaier 1996 \{published data only\}

* Ablassmaier B, Kiessling S, Pier A. "Open" versus laparoscopic appendicectomy ["Offene" versus laparoskopische Appendektomie]. Der Chirurg 1996;67(6):522-5.

Ali 2017 \{published and unpublished data\}

Ali R, Anwar M, Akhtar J. Laparoscopic versus open appendectomy in children: a randomized controlled trial from a developing country. Journal of Pediatric Surgery 2017;53(2):247-9. [PUBMED: 29223666]

Almond 2004 \{published data only\}

* Almond SL, Smith J, Ledwidge N, Khalil BA, Lloyd DA, Baillie C, et al. Prospective comparison of laparoscopic vs. open appendicectomy in the management of childhood appendicitis. British Journal of Surgery. 2004; Vol. 91, issue Suppl:142. 
Alvarado-Aparicio 2003 \{published data only\}

* Alvarado-Aparicio A, Moreno-Portillo M, Periea-Graterol F, Rojano-Rodriguez M, Gonazalez-Monroy L, Palacios, Ruiz A. Laparoscopic appendectomy. Surgical technique description and literature review. Cirugía y Cirujanos 2003;71(6):442-8.

\section{Askarpour 2012 \{published data only\}}

* Askarpour S, Asgari M, Hashemi S, Javaherizadeh H. Changes in levels of c-reactive protein in open appendectomy compared to laparoscopic appendectomy. Przeglad Gastroenterologiczny 2012;7(3):156-60.

\section{Azaro 1999 \{published data only\}}

* Azaro EM, Amaral PC, Ettinger JE, Souza EL, Fortes MF, Alcantara RS, et al. Laparoscopic versus open appendicectomy: a comparative study. Journal of the Society of Laparoendoscopic Surgeons 1999;3:279-83.

\section{Barrat 1998 \{published data only\}}

* Barrat C, Catheline JM, Rizk N, Champault GG. Does laparoscopy reduce the incidence of unnecessary appendicectomies?. Surgical Laparoscopy \& Endoscopy 1999;9(1):27-31.

Barrat C, Champault G, Catheline JM, Rizk N, Ziol M, Guettier C. Does laparoscopy reduce the incidence of useless appendectomies?. Annales de Chirurgie 1998;52(10):965-9.

\section{Borgstein 1997 \{published data only\}}

* Borgstein PJ, Gordijn RV, Eijsbouts QAJ Cuesta MA. Acute appendicitis--a clear-cut case in men, a guessing game in young women. A prospective study on the role of laparoscopy. Surgical Endoscopy 1997;11(9):923-7.

\section{Buanes 1993 \{published data only\}}

Buanes T, Høgevold HE, Ræder MG. Quality assurance in laparoscopic surgery. New methods should be evaluated in prospective comparative studies. [Prospektive sammenliknende studier som kvalitetssikringsredskap: Viktig ved innføring av laparoskopiske teknikker]. Tidsskrift for den Norske Laegeforening 1993;113(16):1978-81.

* Buanes T, Raeder MG. Introduction of laparoscopic technique in gastrointestinal surgery: Experience at a Norwegian university hospital as revealed by prospective comparative studies. Surgical Laparoscopy \& Endoscopy 1993;3(1):21-8.

\section{Busch 2011 \{published data only\}}

Busch M, Gutzwiller FS, Aellig S, Kuettel R, Metzger U, Zingg U. In-hospital delay increases the risk of perforation in adults with appendicitis. World Journal of Surgery 2011;35(7):1626-33. [PUBMED: 21562871]

\section{Champault 1993 \{published data only\}}

* Champault G, Belhassen A, Rizk N, Lauroy J, Vazzana G, Boutelier P. Appendicectomies: MacBurney or laparoscopic? (100 cases) [Appendicectomies MacBurney ou laparoscopie? (100 cas)]. Journal de Chirurgie 1993;130(1):5-8.

\section{Chen 2007 \{published data only\}}

Chen JA, Zhang YD, Thang ZP, Zhang JC, Ding JW, Liang ZH. Laparoscopic versus open appendectomy of acute appendicitis: a random comparison. Journal of Foshan University 2007;25(6):58-61.

\section{Chiarugi 1996 \{published data only\}}

* Chiarugi M, Buccianti P, Celona G, Decanini L, Martino MC, Goletti O, et al. Laparoscopic compared with open appendicectomy for acute appendicitis: a prospective study. European Journal of Surgery 1996;162(5):385-90.

Chiarugi M, Buccianti P, Decanini L, Pucciarelli M, Goletti O, Bertolucci A, et al. Emergency appendicectomy. Comparison of results for laparoscopic versus standard open procedure [Appendicectomia d'urgenza. Confronto tra metodica a cielo aperto e laparoscopica]. Chirurgia 1993;6(9):519-23.

Darzi 1994 \{published data only\}

* Darzi A, Hunt N, Super P, Carey D, Guillou PJ, Monson JR. Laparoscopic appendicectomy. Minimally Invasive Therapy 1994;3(3):163-7.

Decadt 1999 \{published data only\}

* Decadt B, Sussman L, Lewis MP, Secker A, Cohen L, Rogers C, et al. Randomized clinical trial of early laparoscopy in the management of acute non-specific abdominal pain. British Journal of Surgery 1999;86(11):1383-6.

Deutsch 1982 \{published data only\}

* Deutsch AA, Zelikovsky A, Reiss R. Laparoscopy in the prevention of unnecessary appendicectomies: a prospective study. British Journal of Surgery 1982;69(6):336-7.

Fang 2006 \{published data only\}

Fang S, Yu X, Dong Y, Luo H. Contrastive analysis between open appendectomy and laparoscopic appendectomy. Clinical Education of General Practice 2006;2(1):113-5.

Gilchrist 1992 \{published data only\}

* Gilchrist BF, Lobe TE, Schropp KP, Kay GA, Hixson SD, Wrenn EL, et al. Is there a role for laparoscopic appendectomy in pediatric surgery?. Journal of Pediatric Surgery 1992;27(2):209-14.

\section{Hanning 2006 \{published data only\}}

Hanning W, Guoan X, Kaiyun C. A comparative study between laparoscopic and open appendectomy for acute appendicitis in children. Chinese Journal of Minimally Invasive Surgery 2006;6(8):593-4.

Hay 1998 \{published and unpublished data\}

* Hay SA. Laparoscopic versus conventional appendectomy in children. Pediatric Surgery International 1998;13(1):21-3.

Herman 2000 \{published and unpublished data\}

* Herman J, Duda M, Svach I, Stary L. Quo vadis appendectomia laparoscopica? [Quo vadis appendectomia laparoscopica?]. Rozhledy v Chirurgii 2000;79(1):13-6. 
Herman 2003 \{published data only\}

* Herman J, Duda M, Lovecek M, Svach I. Open versus laparoscopic appendectomy. Hepatogastroenterology 2003;50(53):1419-21.

\section{Hill 1991 \{published data only\}}

* Hill AD, Attwood SE, Stephens RB. Laparoscopy appendicectomy is feasible and safe in acute appendicitis. Irish Journal of Medical Science 1991;160(6):268-70.

Jan 2011 \{published data only\}

* Jan WA, Rehman ZU, Khan SM, Ali G, Qayyum A, Mumtaz N. Outcome of open versus laproscopic appendicectomy in department of surgery, Lady Reading Hospital, Peshawar. Journal of Postgraduate Medical Institute 2011;25(3):245-51.

\section{Karaorman 1994 \{published data only\}}

* Karaorman M, Fernandez F, Werthmann K, Trede M. Results of a prospective study on laparoscopic appendicectomy [Ergebnisse einer prospektiven Studie zur laparoskopischen Appendektomie]. Der Chirurg 1994;65(10):1126-9.

\section{Klima 1998 \{published data only\}}

Klima S, Schyra B. Method and results of stump-treatment in laparoscopic appendectomy [Technik und Bedeutung der Stumpfeversorgung für das Ergebnis der laparoskopischen Appendektomie]. Langenbecks Archiv fur Chirurgie 1996;113(Suppl):556-8.

\section{Kollias 1994 \{published data only\}}

* Kollias J, Harries RH, Otto G, Hamilton DW, Cox JS, Gallery RM. Laparoscopic versus open appendicectomy for suspected appendicitis: A prospective study. Australian and New Zealand Journal of Surgery 1994;64(12):830-5.

Koluh 2010 \{published data only\}

Koluh A. Complications during and after laparoscopic appendectomy. Bosnia Herzegovina Surgery 2012;2(1):49-53.

* Koluh A, Delibegovic S, Hasukic S, Valjan V, Latic F. Laparoscopic appendectomy in the treatment of acute appendicitis. Medicinski Arhiv 2010;64(3):147-50. [PUBMED: 20645506]

\section{Konstadoulakis 2006 \{published data only\}}

Konstadoulakis MM, Gomatos IP, Antonakis PT, Manouras A, Albanopoulos K, Nikiteas N, et al. Two-trocar laparoscopicassisted appendectomy versus conventional laparoscopic appendectomy in patients with acute appendicitis. Journal of Laparoendoscopic \& Advanced Surgical Techniques 2006;16(1):27-32.

\section{Kotlobovskii 2003 \{published data only\}}

* Kotlobovskii VI, Dronov AF, Poddubnyi IV, Dzhenalaev BK. Comparative study of surgical and endosurgical treatment of generalized appendicular peritonitis in children [Sravnitel'noe izuchenie rezul'tatov lecheniia rasprostranennykh form appendikuliarnogo peritonita u detei, operirovannykh laparoskopicheskim i traditsionnym khirurgicheskim sposobom]. Khirurgiia 2003;7(1):32-37.
Kum 1993b \{published data only\}

* Kum CK, Sim EK, Goh PM, Ngoi SS, Rauff A. Diagnostic laparoscopy: reducing the number of normal appendectomies. Diseases of the Colon and Rectum 1993;36(8):763-6.

\section{Kumar 2004 \{published data only\}}

Kumar S, Jain S. Treatment of appendiceal mass: prospective, randomized clinical trial. Indian Journal of Gastroenterology 2004;23(5):165-7.

\section{Lamparelli 2000 \{published data only\}}

* Lamparelli MJ, Hoque HM, Pogson CJ, Ball AB. A prospective evaluation of the combined use of the modified alvarado score with selective laparoscopy in adult females in the management of suspected appendicitis. Annals of the Royal College of Surgeons of England 2000;82(3):192-5

\section{Lansdown 1993 \{published data only\}}

* Lansdown M, Kraly Z, Milkins R, Royston C. Conventional versus laparoscopic surgery for acute appendicitis. British Journal of Surgery 1993;80(10):1349-50.

\section{Lau 2005 \{published data only\}}

Lau DH, Yau KK, Chung CC, Leung FC, Tai YP, Li MK. Comparison of needlescopic appendectomy versus conventional laparoscopic appendectomy: a randomized controlled trial. Surgical Laparoscopy, Endoscopy \& Percutaneous Techniques 2005;15(2):75-9.

\section{Li 2005 \{published data only\}}

Li P, Xu Q, Ji Z, Gao Y, Zhang X, Duan Y, et al. A comparative study on surgical stress between laparoscopic and open appendectomy in children.. Chinese Journal of General Surgery 2005;14(1):9.

* Li P, Xu Q, Ji Z, Gao Y, Zhang X, Duan Y, et al. Comparison of surgical stress between laparoscopic and open appendectomy in children. Journal of Pediatric Surgery 2005;40(8):1279-83.

Lujan Mompean 1994 \{published data only\}

* Lujan Mompean JA, Robles Campos R, Parrilla Paricio P, Soria Aledo V, García Ayllon J. Laparoscopic versus open appendicectomy: a prospective assessment. British Journal of Surgery 1994;81(1):133-5.

\section{Malik 2007 \{published data only\}}

Malik AH, Wani RA, Saima BD, Wani MY. Small lateral access-an alternative approach to appendicitis in paediatric patients: $A$ randomised controlled trial. International Journal of Surgery 2007;5:234-8

\section{Malik 2009 \{published data only\}}

Arshad AM, Talpur AH, Laghari AA. Video-assisted laparoscopic extracorporeal appendectomy versus open appendectomy. Journal of Laparoendoscopic \& Advanced Surgical Techniques. Part A 2009;19(3):355-9.

\section{Mantoglu 2013 \{published data only\}}

* Mantoglu B, Okuducu M, Karip AB, Altun H, Onur E, Baskent A, et al. Comparison of hospital costs and clinical data between laparoscopic appendectomy and open appendectomy in a 
developing country. Surgical Endoscopy. 2013; Vol. 27, issue Suppl:S85.

\section{Marzouk 2002 \{published data only\}}

* Marzouk M, Khater M, Elsadek M, Abdelmoghny A. Laparoscopic vs open appendectomy - a prospective comparative study of 227 patients. Surgical endoscopy 2003;17(5):721-4.

\section{McAnena 1992 \{published data only\}}

McAnena OF, Austin O, Hederman WP, Gorey TF, Fitzpatrick J, O'Connell PR. Laparoscopic versus open appendicectomy. Lancet 1991;338(8768):693.

* McAnena OF, Austin O, O'Connell PR, Hederman WP, Gorey TF, Fitzpatrick J. Laparoscopic versus open appendicectomy: A prospective evaluation. British Journal of Surgery 1992;79(8):818-20.

\section{Milewczyk 1998 \{published and unpublished data\}}

* Milewczyk M, Michalik M, Ciesielski M. A prospective, randomized, unicenter study comparing laparoscopic and open treatments of acute appendicitis. Surgical Endoscopy 2003;17(7):1023-8.

Orlowski M, Michalik M, Milewczyk M, Budzinski R, Witzling M, Hochschulz J. Open versus laparoscopic appendectomy comparison of postoperative complications. A randomized prospective study [Porownanie appendektomii laparoskopowej i klasycznej pod wzgledem czestosci wystepowania powiklan pooerayjnych - Praca porspektywna, randomizowana]. Acta Endoscopica Polona 2000;10(1):31-4.

\section{Moberg 1998 \{published data only\}}

* Moberg AC, Ahlberg G, Leijonmarck CE, Montgomery A, Reiertsen O, Rosseland AR, et al. Diagnostic laparoscopy in 1043 patients with suspected acute appendicitis. European Journal of Surgery 1998;164(11):833-41.

\section{Moldovanu 2010 \{published data only\}}

Moldovanu R, Vlad N, Tarcoveanu E, Lupascu C, Dimofte G, Filip V, Bradea C, et al. Acute appendicitis - open versus minimally-invasive approach [abstract]. Surgical Endoscopy. 2010; Vol. 24, issue Suppl:S81-2.

\section{Mugomba 2001 \{published data only\}}

* Mugomba G, Schenk C, Dabidian R, Scheuerecker H, Glaser F. Laparoscopic versus open appendectomy in children - a 2year prospective study [Laparoskopische versus konventionell Appendektomie im Kindesalter - Ergebnisse einer prospektiven 2-Jahres-Beobachtungsstudie]. Zentralblatt für Kinderchirurgie 2001;10(1):16-20.

\section{Näf 1996 \{published data only\}}

* Näf R, Bischofberger U, Buchmann P. Advantages of laparoscopic appendectomy [Avantages de l'appendicectomie laparoscopique]. Revue Medicale de la Suisse Romande 1996;116(7):537-40.

\section{Naver 1994 \{published data only\}}

${ }^{*}$ Naver LP, Kock JP, Bokmand S. Laparoscopic appendicectomy: early experience [Primære erfaringer med laparoskopisk appendektomi]. Ugeskrift for Læger 1994;156(25):3775-7.

Niebuhr 1992 \{published data only\}

* Niebuhr H, Nahrstedt U, Rückert K. Laparoscopic versus conventional appendectomy - which technique in acute appendicitis? [Laparoskopische versus konventionelle Appendektomie - Welches Verfahren bei akuter Appendizitis?]. Laparoendoskopische Chirurgie 1992;1(1):89-97.

Oka 2004 \{published data only\}

* Oka T, Kurkchubasche AG, Bussey JG, Wesselhoeft CW, Tracy TF, Luks Fl. Open and laparoscopic appendectomy are equally safe and acceptable in children. Surgical Endoscopy 2004;18(2):242-5.

Padankatti 2008 \{published data only\}

* Padankatti LR, Kirthy Pramod R, Gupta A, Ramachandran P. Laparoscopic versus open appendicectomy for complicated appendicitis: a prospective study. Journal of Indian Association of Pediatric Surgeons 2008;13(3):104-6.

Planells Roig 1993 \{published data only\}

* Planells Roig MV, García Espinosa R, Moya Sanz A, Rodero D. Laparoscopic appendectomy versus traditional appendectomy: a prospective study of 93 consecutive cases [Apendicectomía laparoscópica frente a apendicectomía tradicional. Estudio prospectivo de 93 casos consecutivos]. Cirugía Española 1994;56(3):208-13.

\section{Prado 1997 \{published data only\}}

* Prado E, García-Alcalá H, Domínguez-Cocco A, JustoJaneiro JM. N/A [Estudio comparativo de apendicectomía laparoscópica con endoasas preatadas vs apendicectomía abierta]. Revista de Gastroenterología de México 1997;62(4):254-9.

\section{Pruett 1994 \{published data only\}}

* Pruett B, Pruett J. Laparoscopic appendectomy: have we found a better way?. Journal of the Mississippi State Medical Association 1994;35(12):347-51.

Raakow 1993 \{published data only\}

* Keck H, Raakow R, Thalmann U, Steinmüller T, Lüsebrink R, Neuhaus P. Results of laparoscopic appendectomy in comparison to conventional surgical technique [Die laparoskopische Appendektomie im Vergleich zur konventionellen Operationstechnik bei akuter Appendizitis]. Acta Chirurgica Austriaca 1995;27(1):55-9.

Raakow R, Keck H, Neuhaus P. Results of laparoscopic appendectomy in comparison to conventional surgical technique [Ergebnisse der laparoskopischen Appendektomie im Vergleich zur konventionellen Operationstechnik]. Langenbecks Archiv für Chirurgie 1993;110(3):180-2.

Rashid 2013 \{published and unpublished data\}

Rashid A, Nazir S, Kakroo SM, Chalkoo MA, Razvi SA, Wani AA. Laparoscopic Interval appendectomy versus open interval appendectomy: a prospective randomized controlled trial. 
Surgical Laparoscopy, Endoscopy \& Percutaneous Techniques 2013;23(1):93-6.

\section{Reiertsen 1994 \{published data only\}}

* Reiertsen O, Trondsen E, Bakka A, Andersen OK, Larsen S, Rosseland AR. Prospective nonrandomized study of conventional versus laparoscopic appendectomy. World Journal of Surgery 1994;18(3):411-6.

\section{Richards 1993 \{published data only\}}

* Richards W, Watson D, Lynch G, Reed GW, Olsen D, Spaw A, et al. A review of the results of laparoscopic versus open appendectomy. Surgery, Gynecology \& Obstetrics 1993;177(5):473-80.

\section{Ritter 1998 \{published data only\}}

* Ritter C, Sahm M, Kubo G. Laparoscopic versus conventional appendectomy [Laparoskopische versus konventionelle Appendektomie]. Zentralblatt für Chirurgie 1998;123:94-6.

\section{Rückert 1993 \{published data only\}}

* Rückert K, Niebuhr H, Nahrstedt U. Laparoscopic appendectomy versus conventional appendectomy in acute appendicitis [Laparoskopische Appendektomie versus konventionelle Appendektomie bei akuter Appendizitis]. Minimal Invasive Chirurgie 1993;2:8.

\section{Sarihan 1994 \{published data only\}}

Sarihan H, Akyazici R, Abes M, Cay A, Mamoglu M. Evaluation of peritoneal drainage in children with perforated appendicitis [Perfore apandisitli cocuklarda peritoneal direnajin degerlendirilmesi]. Pediatrik Cerrahi Dergisi 1994;8(1):26-29.

\section{Sayed Hassen 1996 \{published data only\}}

* Sayed Hassen AH, Cade RJ. A prospective trial of open versus laparoscopic appendicectomy. Australian and New Zealand Journal of Surgery 1996;66(3):178-80.

\section{Schäfer 1997 \{published data only\}}

* Schäfer R, Gerlach K, Barthel M, Schmucker P. Effects of endoscopic operation techniques on respiration in children [Auswirkungen endoskopischer Operationstechniken bei Kindern auf die Beatmung]. Anasthesiologie, Intensivmedizin, Notfallmedizin, Schmerztherapie 1997;32(6):343-7.

\section{Schramm 1994 \{published data only\}}

* Schramm R, Land RD, Unger S, Thiele H. Laparoscopic appendectomy in acute appendicitis [Laparoskopische Appendektomie bei akuter Appendizitis]. Zentralblatt für Chirurgie 1994;111(Suppl):226-8.

\section{Schroder 1993 \{published data only\}}

* Schroder DM, Lathrop JC, Lloyd LR, Boccaccio JE, Hawasli A. Laparoscopic appendectomy for acute appendicitis: is there really any benefit?. American Surgeon 1993;59(8):541-8.

\section{Sfez 1993 \{published data only\}}

* Sfez M. Laparoscopic surgery: the anesthetist's point of view [Cœliochirurgie en pédiatire: le point de vue de l'anesthésiste]. Cahiers d'Anesthésiologie 1993;41(4):237-44.
Shalaby 2001 \{published and unpublished data\}

* Shalaby R, Arnos A, Desoky A, Samaha AH. Laparoscopic appendectomy in children: evaluation of different techniques. Surgical Laparoscopy, Endoscopy \& Percutaneous Techniques 2001;11(1):22-7.

\section{Shen 2012 \{published data only\}}

Shen Z, Ye Y, Yin M, Wang S. Laparoscopic appendectomy for acute appendicitis versus chronic appendicitis. Journal of Investigative Surgery 2012;25(4):209-13.

Shirazi 2010 \{published data only\}

Shirazi B, Ali N, Shamim MS. Laproscopic versus open appendectomy: a comparative study. Journal of the Pakistan Medical Association 2010;60(11):901-4.

Sosa 1993 \{published data only\}

* Sosa JL, Sleeman D, McKenney MG, Dygert J, Yarish D, Martin L. A comparison of laparoscopic and traditional appendectomy. Journal of Laparoendoscopic Surgery 1993;3(2):129-31.

\section{St Peter 2010 \{published data only\}}

St Peter SD, Aguayo P, Fraser JD, Keckler SJ, Sharp SW, Leys CM, et al. Initial laparoscopic appendectomy versus initial nonoperative management and interval appendectomy for perforated appendicitis with abscess: a prospective, randomized trial. Journal of Pediatric Surgery 2010;45(1):236-40.

Tarnoff 1998 \{published data only\}

* Tarnoff M, Atabek U, Goodman M, Alexander JB, Chrzanowski F, Mortman K, et al. A comparison of laparoscopic and open appendectomy. Journal of the Society of Laparoendoscopic Surgeons 1998;2(2):153-8.

Tate 1993b \{published data only\}

* Tate JJ, Chung SC, Dawson J, Leong HT, Chan A, Lau WY, Li AK. Conventional versus laparoscopic surgery for acute appendicitis. British Journal of Surgery 1993;80(6):761-4.

Thon 1996 \{published data only\}

* Thon KP, Stoltzing H. Laparoscopic versus open appendectomy - pleading for distinct decision [Laparoskopische oder konventionelle Appendektomie Plädoyer für eine differenzierte Verfahrenswahl]. Aktuelle Chirurgie 1996;31(4):345-350.

\section{Thorell 1999 \{published data only\}}

* Thorell A, Grondal S, Schedvins K, Wallin G. Value of diagnostic laparoscopy in fertile women with suspected appendicitis. European Journal of Surgery 1999;165(8):751-4.

\section{Till 1994 \{published data only\}}

* Till H, Lochbühler H, Böhm B, Kellnar S. Prospective comparison of the postoperative course following laparoscopic or open appendectomy in the pediatric patient [Prospektive Studie zum postoperativen Verlauf nach laparoskopischer oder konventioneller Appendektomie im Kindesalter]. Langenbecks Archiv für Chirurgie 1994;111:599-602. 
Till H, Weiss M, Kellnar S, Böhm R, Joppich I. Laparoscopic appendectomy reduces post-operative inflammation and shortens convalescence in children [Die laparoskopische Appendektomie verringert im Kindesalter die postoperative Inflammation und verkürzt die Rekonvaleszenz]. Monatsschrift Kinderheilkunde 1995;143:S233.

\section{Ure 1992 \{published data only\}}

* Ure BM, Spangenberger W, Hebebrand D, Eypasch EP, Troidl H. Laparoscopic surgery in children and adolescents with suspected appendicitis: results of medical technology assessment. European Journal of Pediatric Surgery 1992;2(6):336-340.

\section{Van den Broek 2000 \{published data only\}}

* Van den Broek WT, Bijnen AB, Van Eerten PV, de Ruiter P, Gouma DJ. Selective use of diagnostic laparoscopy in patients with suspected appendicitis. Surgical Endoscopy 2000;14(10):938-41.

\section{Varlet 1994 \{published data only\}}

* Varlet F, Tardieu D, Limonne B, Metafiot H, Chavrier Y. Laparoscopic versus open appendectomy in children comparative study of 403 cases. European Journal of Pediatric Surgery 1994;4(6):333-7.

\section{Whitworth 1998 \{published data only\}}

* Whitworth CM, Whitworth PW, Sanfillipo J, Polk HC. Value of diagnostic laparoscopy in young women with possible appendicitis. Surgery, Gynecology \& Obstetrics 1988;167(3):187-90.

\section{Wullstein 2001 \{published data only\}}

* Wullstein C, Barkhausen S, Gross E. Results of laparoscopic vs. conventional appendectomy in complicated appendicitis. Diseases of the Colon and Rectum 2001;44(11):1700-5.

\section{Zaninotto 1995 \{published data only\}}

* Zaninotto G, Rossi M, Anselmino M, Costantino M, Pianalto S, Baldan N, et al. Laparoscopic versus conventional surgery for suspected appendicitis in women. Surgical Endoscopy 1995;9(3):337-40.

\section{References to studies awaiting assessment}

Esposito 1997 \{published data only\}

* Esposito P, Cerbone D, Rotondano G, Del Prete A, Pisano R, Cerbone G. Laparoscopic appendectomy: our experience. Gastrointestinal Endoscopy. 1997; Vol. 45, issue 4:AB186.

\section{Hoff 1995 \{published data only\}}

* Hoff C, Ruers T, Jakimowicz J. Randomized study of laparoscopic versus open appendectomy. Surgical Endoscopy. 1995; Vol. 9, issue 5:605.

\section{Loh 1992 \{published data only\}}

* Loh A, Loosemore TM, Griffiths AB, Fiennes AG, Taylor RS. Less pain and earlier return to work after laparoscopic than after open appendicectomy: a randomized prospective study. 3rd World Congress of Endoscopic Surgery. Bordeaux, France, 1992; Vol. Session CL1/2:Abstract 44.

\section{Rohr 1994 \{published data only\}}

* Rohr S, Thiry CL, de Manzini N, Perrauid V, Meyer C. Laparoscopic vs open appendectomy in men: a prospective randomized study. British Journal of Surgery. 1994; Vol. 81, issue Suppl:6-7.

\section{Talha 2015 \{published data only\}}

Talha Al, Ghazal AH. Laparoscopic vs open management of perforated appendicitis: a prospective randomized clinical trial. Surgical Endoscopy and Other Interventional Techniques. 2015; Vol. 29, issue Suppl:S9.

\section{References to ongoing studies}

ChicTR-TRC-14005067 \{published data only\}

ChiCTR-TRC-14005067. Impact of children laparoscopic appendectomy on gastrointestinal function function: a randomized controlled trial. http://www.chictr.org.cn/ showprojen.aspx?proj=4508 (first received 5 August 2014).

\section{IRCT2015102724747N1 \{published data only\}}

IRCT2015102724747N1. Laparoscopic versus open appendectomy: compare outcomes and complications in the treatment of complicated appendicitis. http://en.irct.ir/ trial/20823 (first received 06 June 2017).

\section{IRCT201703088375N12 \{published data only\}}

IRCT201703088375N12. Comparison of complications and outcomes of three methods of Llparoscopic appendectomy, single-incision and open appendectomy in pediatric patients. http://en.irct.ir/trial/8854 (first received 7 August 2017).

\section{NCT01260064 \{published data only\}}

NCT01260064. Open appendectomy versus laparoscopic appendectomy. clinicaltrials.gov/show/NCT01260064 (first received 15 December 2010).

\section{UMIN000003711 \{published data only\}}

UMIN000003711. Value of laparoscopic appendectomy in perforated appendicitis - a randomized controlled trial. http:// www.umin.ac.jp/ctr/ (first received 18 August 2015).

\section{Additional references}

\section{Addiss 1990}

Addiss DG, Shaffer N, Fowler BS, Tauxe RV. The epidemiology of appendicitis and appendectomy in the United States. American Journal of Epidemiology 1990;132(5):910-25.

\section{Aly 2016}

Aly OE, Black DH, Rehman H, Ahmed I. Single incision laparoscopic appendicectomy versus conventional three-port laparoscopic appendicectomy: a systematic review and metaanalysis. International Journal of Surgery 2017;35:120-8.

\section{ANDEM 1997}

Agence Nationale pour le Développement de l'Évaluation Médicale. Appendicitis: Recommendations and medical references [Appendicite: Recommandations et références 
médicales]. Gastroentérologie Clinique et Biologique 1997;21(1):50-70.

\section{Andreu-Ballester 2009}

Andreu-Ballester JC, Gonzalez-Sanchez A, Ballester F, AlmelaQuilis A, Cano-Cano MJ, Millan-Scheiding M, et al. Epidemiology of appendectomy and appendicitis in the Valencian community (Spain), 1998-2007. Digestive Surgery 2009;26(5):406-12.

\section{Aziz 2006}

Aziz O, Athanasiou T, Tekkis PP, Purkayastha S, Haddow J, Malinovski V, et al. Laparoscopic versus open appendectomy in children: a meta-analysis. Annals of Surgery 2006;243(1):17-27.

\section{Baird 2017}

Baird DL, Similis C, Kontovounisios C, Rasheed S, Tekkis PP. Acute appendicitis. British Medical Association 2017;357:j1703.

\section{Bennett 2007}

Bennett J, Boddy A, Rhodes M. Choice of approach for appendicectomy: a meta-analysis of open versus laparoscopic appendicectomy. Surgical Laparoscopy, Endoscopy \& Percutaneous Techniques 2007;17(4):245-55.

\section{Bregendahl 2013}

Bregendahl S, Nørgaard M, Laurberg S, Jepsen P. Risk of complications and 30-day mortality after laparoscopic and open appendectomy in a Danish region, 1998-2007; a populationbased study of 18,426 patients. Polski Przeglad Chirurgiczny 2013;85(7):395-400.

\section{Bulian 2013}

Bulian DR, Knuth J, Sauerwald A, Strohlein MA, Lefering R, Ansorg J, et al. Appendectomy in Germany-an analysis of a nationwide survey 2011/2012. International Journal of Colorectal Disease 2013;28(1):127-38.

\section{Cheng 2015}

Cheng Y, Zhou S, Zhou R, Lu J, Wu S, Xiong X, et al. Abdominal drainage to prevent intra-peritoneal abscess after open appendectomy for complicated appendicitis. Cochrane Database of Systematic Reviews 2015, Issue 2. [DOI: 10.1002/14651858.CD010168.pub2]

\section{Chung 1999}

Chung RS, Rowland DY, Li P, Diaz J. A meta-analysis of randomized controlled trials of laparoscopic versus conventional appendectomy. American Journal of Surgery 1999;177:250-6.

\section{Dai 2017}

Dai L, Shuai J. Laparoscopic versus open appendectomy in adults and children: a meta-analysis of randomized controlled trials. United European Gastroenterology Journal 2017;5(4):542-53. [PUBMED: 28588886]

\section{Deeks 2011}

Deeks JJ, Higgins JP, Altman DG. Chapter 9: Analysing data and undertaking meta-analyses. In: Higgins JP, Green S, editor(s). Cochrane Handbook for Systematic Reviews of
Interventions Version 5.1.0 (updated March 2011). The Cochrane Collaboration, 2011. Available from handbook.cochrane.org.

\section{Egger 1997}

Egger M, Smith G, Schneider M, Minder C. Bias in meta-analysis detected by a simple, graphical test. British Medical Journal 1997;315(7109):629-34.

\section{Eriksson 1995}

Eriksson S, Granström L. Randomized controlled trial of appendicectomy versus antibiotic therapy for acute appendicitis. British Journal of Surgery 1995;82(2):166-9.

\section{Eypasch 1996}

Eypasch E, Felsenstein M, Köhler L, Troidl H. Decision-making in acute appendicitis: meta-analysis versus clinical practice. Digestive Surgery 1996;13(4-5):309-13.

\section{Faiz 2008}

Faiz O, Clark J, Brown T, Bottle A, Antoniou A, Farrands P, et al. Traditional and laparoscopic appendectomy in adults: outcomes in English NHS hospitals between 1996 and 2006. Annals of Surgery 2008;248(5):800-6

\section{Fingerhut 1999}

Fingerhut A, Millat B, Borrie F. Laparoscopic versus open appendectomy: time to decide. World Journal of Surgery 1999;23(8):835-45.

\section{Follmann 1992}

Follmann D, Elliot P, Suh I, Cutler J. Variance imputation for overviews of clinical trials with continuous response. Journal of Clinical Epidemiology 1992;45(7):769-73.

\section{Garbutt 1999}

Garbutt JM, Soper NJ, Shannon WD, Botero A, Littenberg B. Meta-analysis of randomized controlled trials comparing laparoscopic and open appendectomy. Surgical Laparoscopy and Endoscopy 1999;9:17-26.

\section{Golub 1998}

Golub R, Siddiqui F, Pohl D. Laparoscopic versus open appendectomy: a metaanalysis. Journal of the American College of Surgeons 1998;186(5):545-53.

\section{Higgins 2011}

Higgins JP, Green S (editors). Cochrane Handbook for Systematic Reviews of Interventions Version 5.1.0 (updated March 2011). The Cochrane Collaboration, 2011. Available from handbook.cochrane.org.

\section{Ingraham 2010}

Ingraham AM, Cohen ME, Bilimoria KY, Pritts TA, Ko CY, Esposito TJ. Comparison of outcomes after laparoscopic versus open appendectomy for acute appendicitis at 222 ACS NSQIP hospitals. Surgery 2010;148(4):625-35. [PUBMED: 20797745]

\section{Jaschinski 2015}

Jaschinski T, Mosch C, Eikermann M, Neugebauer EA. Laparoscopic versus open appendectomy in patients with 
suspected appendicitis: a systematic review of meta-analyses of randomised controlled trials. BMC Gastroenterology 2015;15:48.

\section{Jeon 2017}

Jeon BG. Predictive factors and outcomes of negative appendectomy. American Journal of Surgery 2017;213(4):731-8. [PUBMED: 27769547]

\section{Kapischke 2006}

Kapischke M, Caliebe A, Tepel J, Schulz T, Hedderich J. Open versus laparoscopic appendicectomy: a critical review. Surgical Endoscopy 2006;20(7):1060-8.

\section{Kapischke 2011}

Kapischke M, Friedrich F, Hedderich J, Schulz T, Caliebe A. Laparoscopic versus open appendectomy--quality of life 7 years after surgery. Langenbeck's archives of surgery / Deutsche Gesellschaft fur Chirurgie 2011;396(1):69-75. [PUBMED: 20927534]

\section{Kim 2004}

Kim CB, Kim MS, Hong J, Lee HY, Yu SH. Is laparoscopic appendectomy useful for the treatment of acute appendicitis in Korea? A meta-analysis. Yonsei Medical Journal 2004;45(1):7-16.

\section{Kotaluoto 2017}

Kotaluoto S, Ukkonen M, Pauniaho SL, Helminen M, Sand J, Rantanen T. Mortality related to appendectomy: a population based analysis over two decades in Finland. World Journal of Surgery 2017;41(1):64-9. [PUBMED: 27535664]

\section{Lee 2010}

Lee JH, Park YS, Choi JS. The epidemiology of appendicitis and appendectomy in South Korea: national registry data. Journal of epidemiology / Japan Epidemiological Association 2010;20(2):97-105.

\section{Li 2010}

Li X, Zhang J, Sang L, Zhang W, Chu Z, Li X, et al. Laparoscopic versus conventional appendectomy - a meta-analysis of randomized controlled trials. BMC Gastroenterology 2010;10:129.

\section{Lin 2015}

Lin KB, Lai KR, Yang NP, Wu KS, Ting HW, Pan RH, et al. Trends and outcomes in the utilization of laparoscopic appendectomies in a low-income population in Taiwan from 2003 to 2011. International Journal for Equity in Health 2015;14(100):1-10.

\section{Liu 2010}

Liu Z, Zhang P, Ma Y, Chen H, Zhou Y, Zhang M, et al. Laparoscopy or not: a meta-analysis of the surgical effects of laparoscopic versus open appendicectomy. Surgical Laparoscopy, Endoscopy and Percutaneous Techniques 2010;20(6):362-70.

\section{Majeed 1996}

Majeed AW, Troy G, Nicholl JP, Smythe A, Reed MW, Stoddard CJ, et al. Randomised, prospective, single-blind comparison of laparoscopic versus small-incision cholecystectomy. Lancet 1996;347(9007):989-94.

\section{Mannu 2017}

Mannu GS, Sudul MK, Bettencourt-Silva JH, Cumber E, Li F, Clark $A B$, et al. Closure methods of the appendix stump for complications during laparoscopic appendectomy. Cochrane Database of Systematic Reviews 2017, Issue 11. [DOI: 10.1002/14651858.CD006437.pub3]

\section{Markar 2014}

Markar SR, Penna M, Harris A. Laparoscopic approach to appendectomy reduces the incidence of short- and longterm post-operative bowel obstruction: systematic review and pooled analysis. Journal of Gastrointestinal Surgery 2014;18(9):1683-92.

\section{McBurney 1894}

McBurney $C$. The incision made in the abdominal wall in cases of appendicitis, with a description of a new method of operating. Annals of Surgery 1894;20(1):38.

\section{Meynaud-Kraemer 1999}

Meynaud-Kraemer L, Colin C, Vergnon P, Barth X. Wound infection in open versus laparoscopic appendectomy - a metaanalysis. International Journal of Technology Assessment in Health Care 1999;15(2):380-91.

\section{Neugebauer 1991}

Neugebauer E, Troidl H, Spangenberger W, Dietrich A, Lefering R. Conventional versus laparoscopic cholecystectomy and the randomized controlled trial. British Journal of Surgery 1991;78(2):150-4.

\section{Neugebauer 1999}

Neugebauer E. Resolution of problem needed. American Journal of Surgery 1999;177(6):525.

\section{Ohtani 2012}

Ohtani H, Tamamori Y, Arimoto Y, Nishiguchi Y, Maeda K, Hirakawa K. Meta-analysis of the results of randomized controlled trials that compared laparoscopic and open surgery for acute appendicitis. Journal of Gastrointestinal Surgery 2012;16(10):1929-39.

\section{Paterson 2008}

Paterson HM, Qadan M, de Luca SM, Nixon SJ, PatersonBrown S. Changing trends in surgery for acute appendicitis. British Journal of Surgery 2008;95(3):363-8.

\section{Rehman 2011}

Rehman H, Rao AM, Ahmed I. Single incision versus conventional multi-incision appendicectomy for suspected appendicitis. Cochrane Database of Systematic Reviews 2011, Issue 7. [DOI: 10.1002/14651858.CD009022.pub2]

\section{Review Manager 2014 [Computer program]}

Nordic Cochrane Centre, The Cochrane Collaboration. Review Manager 5 (RevMan 5). Copenhagen: Nordic Cochrane Centre, The Cochrane Collaboration, 2014. 


\section{Sadr-Azodi 2009}

Sadr-Azodi O, Andren-Sandberg A. The quality of randomized clinical trials in the field of surgery: studies on laparoscopic versus open appendectomy as an example. Digestive Surgery 2009;26(5):351-7.

\section{Semm 1983}

Semm K. Endoscopic appendectomy. Endoscopy 1983;15(2):59-64.

\section{Slim 1998}

Slim K, Pezet D, Chipponi J. Laparoscopic or open appendectomy? Critical review of randomized, controlled trials. Diseases of the Colon and Rectum 1998;41(3):398-403.

\section{Temple 1999}

Temple LK, Litwin DE, McLeod RS. A meta-analysis of laparoscopic versus open appendectomy in patients suspected of having acute appendicitis. Canadian Journal of Surgery 1999;42(5):377-83.

\section{Tzovaras 2007}

Tzovaras G, Liakou P, Baloyiannis I, Spyridakis M, Mantzos F, Tepetes K, et al. Laparoscopic appendectomy: differences between male andfemale patients with suspected acute appendicitis. World Journal of Surgery 2007;31(2):409-13.

\section{Ukai 2016}

Ukai T, Shikata S, Takeda H, Dawes L, Noguchi Y, Nakayama T, et al. Evidence of surgical outcomes fluctuates over time: results from a cumulative meta-analysis of laparoscopic versus open appendectomy for acute appendicitis. BMC Gastroenterology 2016;16:37.

\section{Van Hove 2008}

Van Hove C, Hardiman K, Diggs B, Deveney C, Sheppard B. Demographic and socioeconomic trends in the use of laparoscopic appendectomy from 1997 to 2003. American Journal of Surgery 2008;195(5):580-4.

\section{Van Rossem 2016}

Van Rossem CC, Bolmers MD, Schreinemacher MH, Van Geloven AA, Bemelman WA. Prospective nationwide outcome audit of surgery for suspected acute appendicitis. British Journal of Surgery 2016;103(1):144-51.

\section{Webb 2011}

Webb EM, Nguyen A, Wang ZJ, Stengel JW, Westphalen AC, Coakley FV. The negative appendectomy rate: who benefits

\section{CHARACTERISTICS OF STUDIES}

Characteristics of included studies [ordered by study ID] from preoperative CT?. American Journal of Roentgenology 2011;197(4):861-6. [PUBMED: 21940573]

\section{Wei 2011}

Wei B, Qi CL, Chen TF, Zheng ZH, Huang JL, Hu BG, et al. Laparoscopic versus open appendectomy for acute appendicitis: a metaanalysis. Surgical Endoscopy 2011;25(4):1199-208.

\section{Weiser 2015}

Weiser TG, Uribe-Leitz T, Fu R, Jaramillo J, Maurer L, Esquivel MM, et al. Variability in mortality after caesarean delivery, appendectomy, and groin hernia repair in low-income and middle-income countries: implications for expanding surgical services. Lancet 2015;385:34.

\section{Wilms 2011}

Wilms IM, de Hoog DE, de Visser DC, Janzing HM. Appendectomy versus antibiotic treatment for acute appendicitis. Cochrane Database of Systematic Reviews 2011, Issue 11. [DOI: 10.1002/14651858.CD004577.pub3]

\section{References to other published versions of this review \\ Sauerland 1998}

Sauerland S, Lefering R, Holthausen U, Neugebauer EAM. Laparoscopic vs conventional appendectomy - a meta-analysis of randomised controlled trials. Langenbeck's archives of surgery 1998;383(3-4):289-95.

\section{Sauerland 2002}

Sauerland S, Lefering R, Neugebauer EAM. Laparoscopic versus open surgery for suspected appendicitis. Cochrane Database of Systematic Reviews 2002, Issue 10. [DOI: 10.1002/14651858.CD001546]

\section{Sauerland 2004}

Sauerland S, Lefering R, Neugebauer EAM. Laparoscopic versus open surgery for suspected appendicitis. Cochrane Database of Systematic Reviews 2004, Issue 10. [DOI: 10.1002/14651858.CD001546.pub2]

\section{Sauerland 2010}

Sauerland S, Jaschinski T, Neugebauer EAM. Laparoscopic versus open surgery for suspected appendicitis. Cochrane Database of Systematic Reviews 2010, Issue 10. [DOI: 10.1002/14651858.CD001546.pub3; PUBMED: 20927725]

* Indicates the major publication for the study

Aktimur 2016

$\begin{array}{ll}\text { Methods } & \text { Randomised controlled trial } \\ \text { Centres: one (Turkey) }\end{array}$


Aktimur 2016 (Continued)

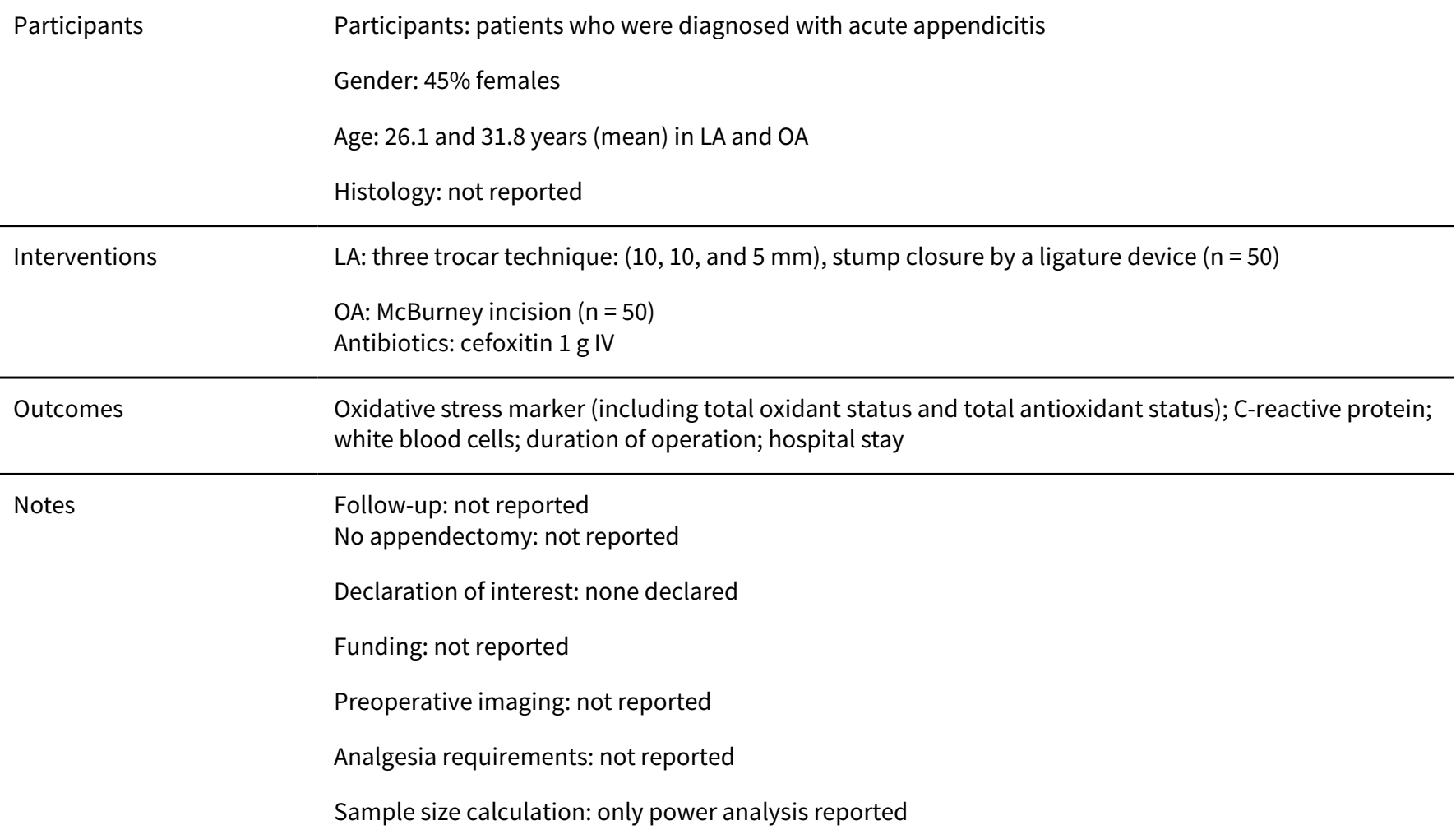

\section{Risk of bias}

\begin{tabular}{lll}
\hline Bias & Authors' judgement & Support for judgement \\
\hline $\begin{array}{l}\text { Random sequence genera- } \\
\text { tion (selection bias) }\end{array}$ & Low risk & $\begin{array}{l}\text { "Just before the surgery patients were assigned to the LA or OA group by } \\
\text { means of sealed envelopes containing random numbers." }\end{array}$ \\
\hline $\begin{array}{l}\text { Allocation concealment } \\
\text { (selection bias) }\end{array}$ & Low risk & $\begin{array}{l}\text { "Just before the surgery patients were assigned to the LA or OA group by } \\
\text { means of sealed envelopes containing random numbers." }\end{array}$ \\
\hline $\begin{array}{l}\text { Blinding of participants } \\
\text { and personnel (perfor- } \\
\text { mance bias) }\end{array}$ & Unclear risk & The trial was described as "double-blind". But further descriptions are missing. \\
All outcomes & \\
\hline
\end{tabular}

\begin{tabular}{|c|c|c|}
\hline $\begin{array}{l}\text { Blinding of outcome as- } \\
\text { sessment (detection bias) } \\
\text { All outcomes }\end{array}$ & Unclear risk & The trial was described as "double-blind". But further descriptions are missing. \\
\hline
\end{tabular}

\begin{tabular}{lll}
\hline $\begin{array}{l}\text { Incomplete outcome data } \\
\text { (attrition bias) } \\
\text { All outcomes }\end{array}$ & Unclear risk & There was no information on conversions, analyses or losses to follow-up. \\
\hline $\begin{array}{l}\text { Selective reporting (re- } \\
\text { porting bias) }\end{array}$ & Unclear risk & No distinction between primary and secondary outcomes \\
\hline $\begin{array}{l}\text { Other bias } \\
\text { Low risk }\end{array}$ & $\begin{array}{l}\text { "All the operations were performed by stuff surgeons of our institutions, who } \\
\text { were experienced in laparoscopic and open surgical techniques." }\end{array}$ \\
\hline
\end{tabular}


Al-Mulhim 2002

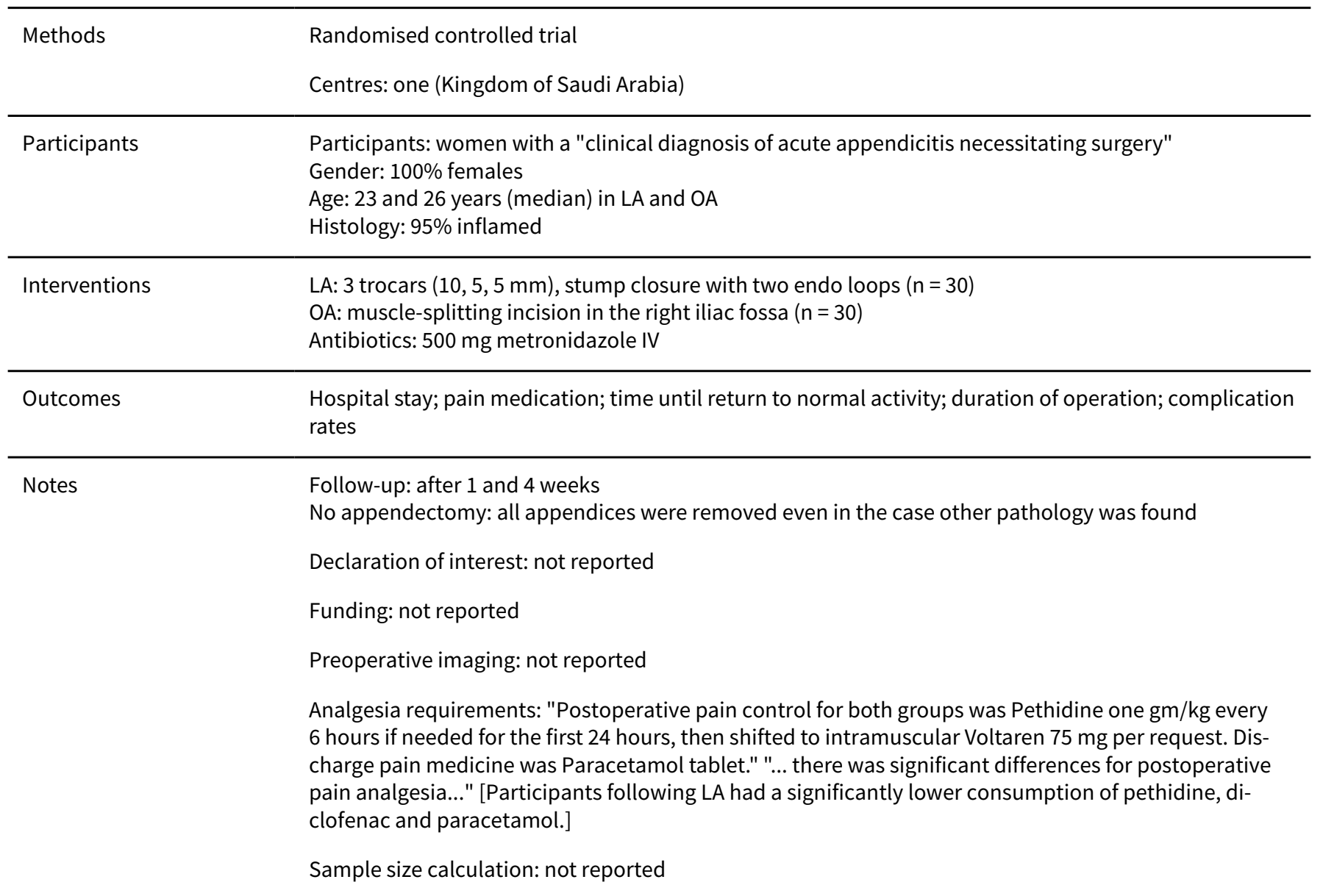

\section{Risk of bias}

\begin{tabular}{|c|c|c|}
\hline Bias & Authors' judgement & Support for judgement \\
\hline $\begin{array}{l}\text { Random sequence genera- } \\
\text { tion (selection bias) }\end{array}$ & Unclear risk & "[Patients] were randomised..." \\
\hline $\begin{array}{l}\text { Allocation concealment } \\
\text { (selection bias) }\end{array}$ & Low risk & "...using a sealed envelope system" \\
\hline $\begin{array}{l}\text { Blinding of participants } \\
\text { and personnel (perfor- } \\
\text { mance bias) } \\
\text { All outcomes }\end{array}$ & Unclear risk & Not reported \\
\hline $\begin{array}{l}\text { Blinding of outcome as- } \\
\text { sessment (detection bias) } \\
\text { All outcomes }\end{array}$ & Unclear risk & Not reported \\
\hline $\begin{array}{l}\text { Incomplete outcome data } \\
\text { (attrition bias) } \\
\text { All outcomes }\end{array}$ & Unclear risk & $\begin{array}{l}\text { No reporting of number and reasons for exclusions or completeness of fol- } \\
\text { low-up. Analyses appeared to be performed on an ITT basis (including three } \\
\text { converted cases in the original group). }\end{array}$ \\
\hline $\begin{array}{l}\text { Selective reporting (re- } \\
\text { porting bias) }\end{array}$ & Unclear risk & No distinction between primary and secondary outcome \\
\hline
\end{tabular}


Al-Mulhim 2002 (Continued)

Other bias Low risk Surgeons "were experienced in laparoscopic surgery".

Attwood 1992

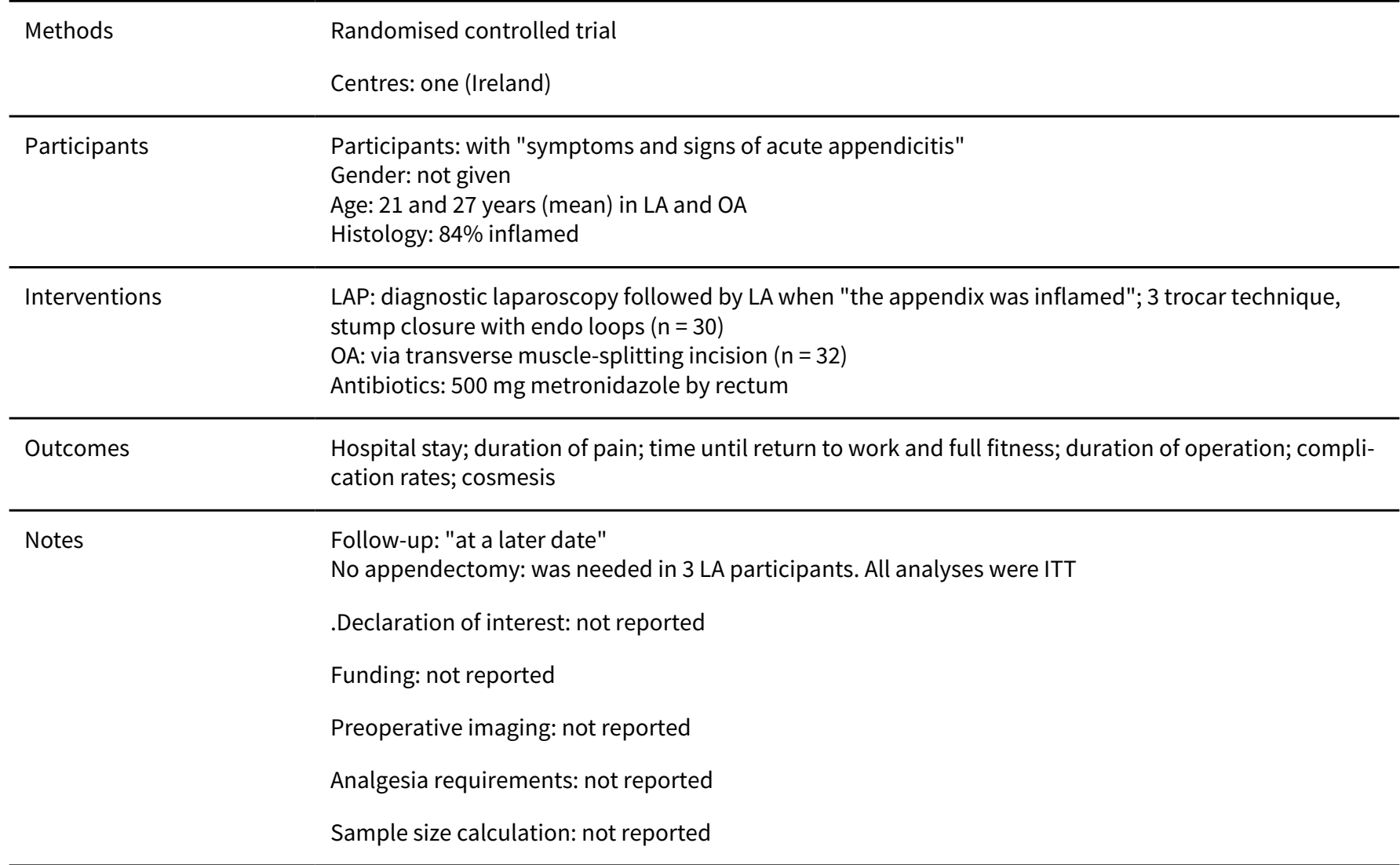

\section{Risk of bias}

\begin{tabular}{lll}
\hline Bias & Authors' judgement & Support for judgement \\
\hline $\begin{array}{l}\text { Random sequence genera- } \\
\text { tion (selection bias) }\end{array}$ & Unclear risk & "were randomised" \\
\hline $\begin{array}{l}\text { Allocation concealment } \\
\text { (selection bias) }\end{array}$ & Low risk & "random drawing of envelopes" \\
\hline $\begin{array}{l}\text { Blinding of participants } \\
\text { and personnel (perfor- } \\
\text { mance bias) }\end{array}$ & Unclear risk & Not reported \\
$\begin{array}{l}\text { All outcomes } \\
\text { Blinding of outcome as- } \\
\text { sessment (detection bias) } \\
\begin{array}{l}\text { All outcomes } \\
\text { Incomplete outcome data }\end{array}\end{array}$ & Unclear risk & \\
\hline $\begin{array}{l}\text { (attrition bias) } \\
\text { All outcomes }\end{array}$ & Not reported \\
\hline
\end{tabular}


Attwood 1992 (Continued)

Selective reporting (re- Unclear risk No distinction between primary and secondary outcome porting bias)

Other bias

High risk

Learning curve bias can be assumed, because "the time taken to perform LA steadily declined during the course of the study."

Barth 1999

\begin{tabular}{ll}
\hline Methods & Randomised controlled trial \\
& Centres: one (?) (France) \\
\hline Participants & $\begin{array}{l}\text { Participants: not reported } \\
\text { Gender: } 50 \% \text { females } \\
\text { Age: } 25 \text { years (mean) } \\
\text { Histology: not reported }\end{array}$ \\
\hline Interventions & LA: not reported \\
& Antibiotics: not reported \\
\hline Outcomes & Complication rates \\
\hline Notes & Follow-up: not reported \\
& No appendectomy: not reported \\
& Declaration of interest: not reported \\
& Funding: not reported \\
& Preoperative imaging: not reported \\
Analgesia requirements: not reported & Sample size calculation: not reported \\
&
\end{tabular}

\section{Risk of bias}

\begin{tabular}{lll}
\hline Bias & Authors' judgement & Support for judgement \\
\hline $\begin{array}{l}\text { Random sequence genera- } \\
\text { tion (selection bias) }\end{array}$ & Unclear risk & Not reported \\
\hline $\begin{array}{l}\text { Allocation concealment } \\
\text { (selection bias) }\end{array}$ & Unclear risk & Not reported \\
\hline $\begin{array}{l}\text { Blinding of participants } \\
\text { and personnel (perfor- } \\
\text { mance bias) }\end{array}$ & Unclear risk & Not reported \\
All outcomes & \\
\hline
\end{tabular}

\begin{tabular}{lll}
\hline $\begin{array}{l}\text { Blinding of outcome as- } \\
\text { sessment (detection bias) } \\
\text { All outcomes }\end{array}$ & Unclear risk & Not reported \\
\hline $\begin{array}{l}\text { Incomplete outcome data } \\
\text { (attrition bias) }\end{array}$ & Unclear risk & $\begin{array}{l}\text { No information on conversions. No reporting of number and reasons for exclu- } \\
\text { sions or completeness of follow-up. }\end{array}$
\end{tabular}


Barth 1999 (Continued)

All outcomes

\begin{tabular}{|c|c|c|}
\hline $\begin{array}{l}\text { Selective reporting (re- } \\
\text { porting bias) }\end{array}$ & Unclear risk & $\begin{array}{l}\text { Only one outcome was reported, but the trial was reported only in form of a } \\
\text { systematic review. }\end{array}$ \\
\hline
\end{tabular}

Other bias Unclear risk The surgeon's skills were not described.

Bartin 2016

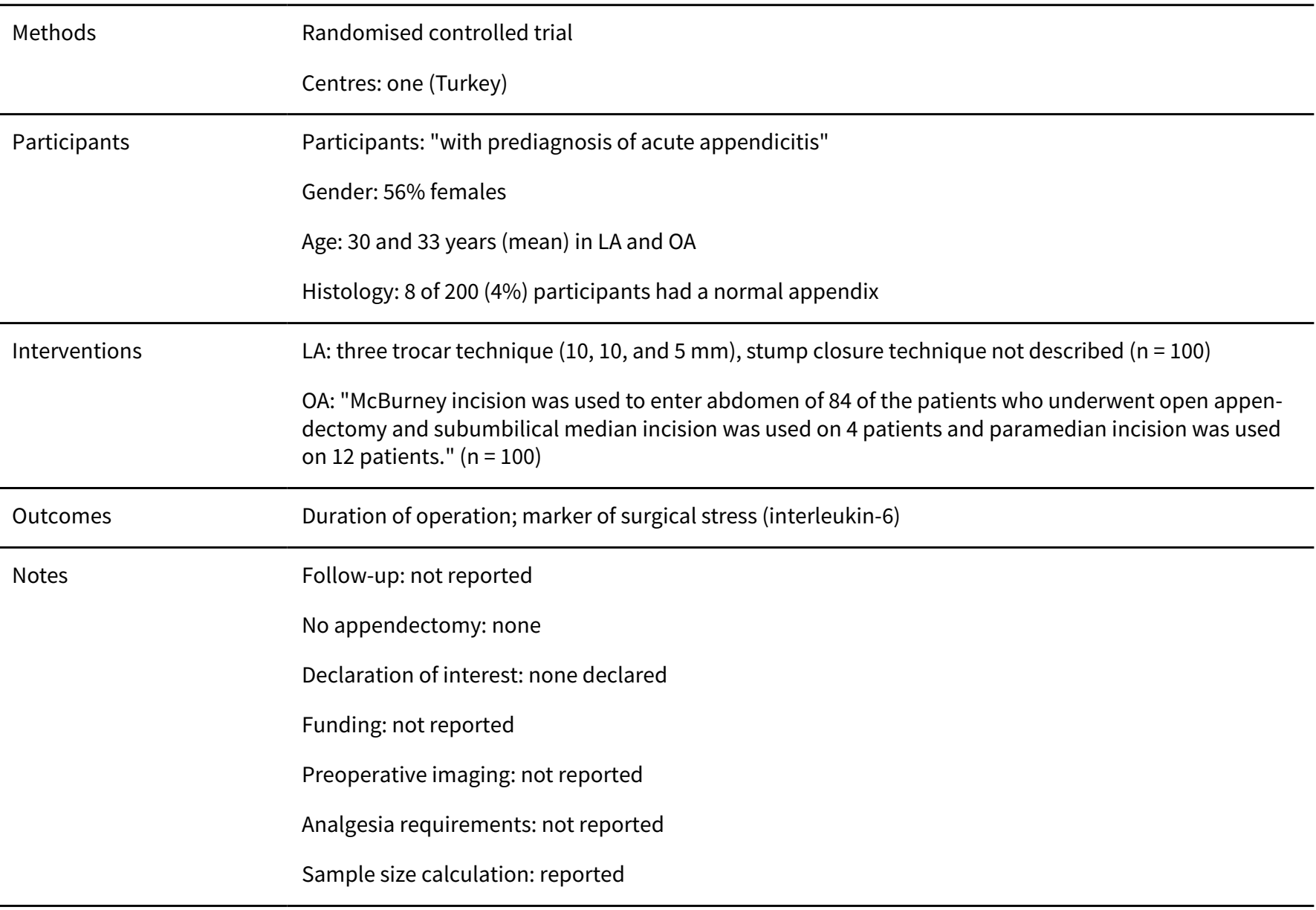

\section{Risk of bias}

\begin{tabular}{lll}
\hline Bias & Authors' judgement & Support for judgement \\
\hline $\begin{array}{l}\text { Random sequence genera- } \\
\text { tion (selection bias) }\end{array}$ & Unclear risk & "After preoperative randomization..." \\
\hline $\begin{array}{l}\text { Allocation concealment } \\
\text { (selection bias) }\end{array}$ & Unclear risk & Not reported \\
\hline $\begin{array}{l}\text { Blinding of participants } \\
\text { and personnel (perfor- } \\
\text { mance bias) }\end{array}$ & Unclear risk & Not reported \\
All outcomes & \\
\hline
\end{tabular}


Bartin 2016 (Continued)
Blinding of outcome as-
Unclear risk
Not reported sessment (detection bias)

All outcomes

\begin{tabular}{|c|c|c|}
\hline $\begin{array}{l}\text { Incomplete outcome data } \\
\text { (attrition bias) }\end{array}$ & Low risk & $\begin{array}{l}\text { No losses from follow-up visible. No conversions or other changes in surgical } \\
\text { procedure }\end{array}$ \\
\hline
\end{tabular}

All outcomes procedure

Selective reporting (re- Unclear risk $\quad$ No distinction between primary and secondary outcome
porting bias)

\begin{tabular}{ll}
\hline Other bias $\quad$ Unclear risk $\quad$ The surgeon's skills were not described. \\
\hline
\end{tabular}

Bauwens 1999

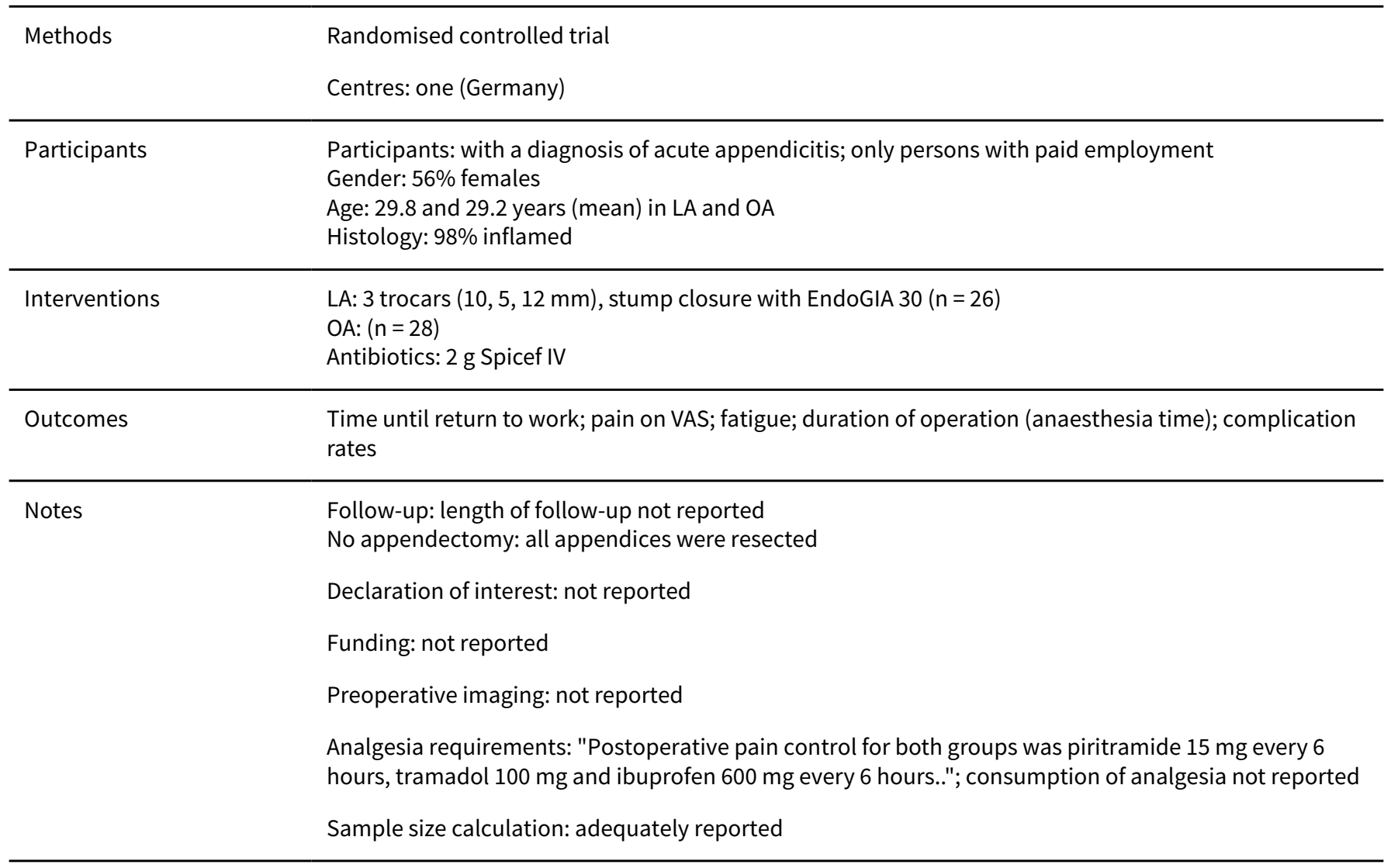

\section{Risk of bias}

Bias Authors' judgement Support for judgement

Random sequence genera- Unclear risk Block randomisation stratified for sex and age ( $<=50$ years, $>50$ years) tion (selection bias)

$\begin{array}{ll}\begin{array}{l}\text { Allocation concealment } \\ \text { (selection bias) }\end{array} & \text { Low risk } \\ \end{array}$




\section{Bauwens 1999 (Continued)}

Blinding of participants and personnel (perfor-

Unclear risk Not reported mance bias)

All outcomes

\begin{tabular}{|c|c|c|}
\hline $\begin{array}{l}\text { Blinding of outcome as- } \\
\text { sessment (detection bias) } \\
\text { All outcomes }\end{array}$ & Unclear risk & Not reported \\
\hline
\end{tabular}

\section{Incomplete outcome data High risk} (attrition bias)

All outcomes
Five of the 59 participants randomised were lost to follow-up and excluded from all analyses. The one converted case was analysed by ITT.

Selective reporting (re- Low risk Primary outcome (time until return to work) was adequately described.
porting bias)

porting bias)

Other bias Low risk Experience with > 20 LAs was required for trial surgeons.

Bliss 2014

\begin{tabular}{ll}
\hline Methods & Randomised controlled trial \\
& Centres: two (USA) \\
\hline
\end{tabular}

Participants

Participants: "children 0-18 years old with appendicitis"

Gender: 39\% females

Age: 9.6 and 9.7 years (mean) in LA and OA

Histology: not reported

LA: three trocar technique $(12,5$, and $5 \mathrm{~mm})$, stump closure technique not described $(\mathrm{n}=177)$
OA: right lower quadrant open incision $(\mathrm{n}=206)$
Antibiotics:
- all children preoperatively: second-generation cephalosporin (cefotetan or cefoxitin) IV
- only children labelled as having complicated appendicitis: additionally ampicillin, ceftazidime and
clindamycin IV

Outcomes Primary outcomes: wound infection; intra-abdominal abscess

Further outcomes: time to tolerate first oral intake; post-anaesthesia recovery room pain medication; inpatient intravenous and oral opioid doses; inpatient nonsteroidal anti-inflammatory medication doses; outpatient oral opioid and non-opioid doses; pain scores (VAS); length of hospital stay; parental estimate of total time to participant recovery; participant and parental satisfaction scores (5-point scale after 7 and 30 days); visits to other healthcare providers; visits to the emergency department; reoperation or re-intervention; wound hematoma; urinary tract infection; pneumonia; bowel obstruction; diarrhoea

Follow-up: 7 and 30 days postoperatively
No appendectomy: none reported
Declaration of interest: none of the authors had anything to disclose
Funding: Oregon Health and Science University


Bliss 2014 (Continued)

Preoperative imaging: not reported

Analgesia requirements: not reported

Sample size calculation: partly reported

\section{Risk of bias}

\begin{tabular}{lll}
\hline Bias & Authors' judgement & Support for judgement \\
\hline $\begin{array}{l}\text { Random sequence genera- } \\
\text { tion (selection bias) }\end{array}$ & Low risk & $\begin{array}{l}\text { "Randomization was performed using block technique. An independent sta- } \\
\text { tistician with no patient contact and with access only to de-identified data at } \\
\text { programmed, periodic reviews, generated blocks of 6-10 sealed envelopes per } \\
\text { round for each institution." }\end{array}$ \\
& & \\
&
\end{tabular}

Allocation concealment Low risk (selection bias)

\begin{abstract}
"These were numbered sequentially and stored in a single location at each institution. The randomisation envelope and relevant surgical instruments were opened only once the child had undergone induction of general anesthesia."
\end{abstract}

Blinding of participants Low risk and personnel (perfor-

"... healthcare team, family, and data collectors remained blinded to the operation performed until post-operative day $7 . "$

mance bias)

All outcomes

"The double dressings were designed to minimize unblinding due to leaking of wound fluid through the first gauze. Four total dressings were placed to hide the type of operation..."

"Documented or potential unblinding such as wound fluid strike-through or dressing dislodgement occurred in $17(10 \%)$ of the laparoscopic group and 13 (6\%) of the open group."

Blinding of outcome as- Low risk sessment (detection bias)

All outcomes

\begin{abstract}
"... healthcare team, family, and data collectors remained blinded to the operation performed until post-operative day $7 . "$

"The double dressings were designed to minimize unblinding due to leaking of wound fluid through the first gauze. Four total dressings were placed to hide the type of operation..."

"Documented or potential unblinding such as wound fluid strike-through or dressing dislodgement occurred in $17(10 \%)$ of the laparoscopic group and 13 (6\%) of the open group."
\end{abstract}

There were no conversions and analyses were done following the ITT principle. Losses to follow-up occurred in 2 of 177 (LA) and 1 of 206 (OA).

Data on main results appeared to be complete.

Selective reporting (re- Low risk porting bias)

Incomplete outcome data Low risk (attrition bias)

All outcomes$$
\text { Losses to follow-up occurredin } 2 \text { of } 177 \text { (LA) and } 1 \text { of } 206 \text { (OA). }
$$

Other bias Unclear risk "The standardized open surgical technique coupled with operative times that were nearly identical to laparoscopy suggested that neither variation in surgical method or expertise was a contributor. Alternatively, the presence of different level trainees may have impacted operative outcomes."

$\begin{array}{ll}\text { Methods } & \text { Randomised controlled trial } \\ \text { Centres: one (Italy) }\end{array}$


Bolla 2008 (Continued)

\begin{tabular}{ll} 
Participants & Participants: children "treated for acute appendicitis, with or without localized peritonitis" \\
Gender: $53 \%$ females & $\begin{array}{l}\text { Age: } 6 \text { years (mean) } \\
\text { Histology: not reported }\end{array}$ \\
\hline Interventions & LA: "three access points: umbilical, hypogastric and left iliac", trocar sizes and dissection technique for \\
appendix stump not described $(n=20)$ & $\begin{array}{l}\text { OA: McBurney incision }(n=20) \\
\text { Antibiotics: cephalosporin, metronidazole }\end{array}$ \\
\hline Outcomes & Wound infection; duration of operation; immunological markers \\
\hline Notes & $\begin{array}{l}\text { Follow-up: not reported } \\
\text { No appendectomy: not reported }\end{array}$ \\
& Declaration of interest: not reported \\
& Funding: not reported \\
& Preoperative imaging: not reported \\
& Analgesia requirements: not reported \\
& Sample size calculation: not reported
\end{tabular}

\section{Risk of bias}

\begin{tabular}{lll}
\hline Bias & Authors' judgement & Support for judgement \\
\hline $\begin{array}{l}\text { Random sequence genera- } \\
\text { tion (selection bias) }\end{array}$ & Unclear risk & "[The 40 children] were randomly allocated..." \\
\hline $\begin{array}{l}\text { Allocation concealment } \\
\text { (selection bias) }\end{array}$ & Unclear risk & Not reported \\
\hline $\begin{array}{l}\text { Blinding of participants } \\
\begin{array}{l}\text { and personnel (perfor- } \\
\text { mance bias) }\end{array}\end{array}$ & Unclear risk & Not reported \\
All outcomes & \\
\hline
\end{tabular}

\begin{tabular}{|c|c|c|}
\hline $\begin{array}{l}\text { Blinding of outcome as- } \\
\text { sessment (detection bias) } \\
\text { All outcomes }\end{array}$ & Unclear risk & Not reported \\
\hline $\begin{array}{l}\text { Incomplete outcome data } \\
\text { (attrition bias) } \\
\text { All outcomes }\end{array}$ & Unclear risk & $\begin{array}{l}\text { No reporting of number and reasons for exclusions or completeness of fol- } \\
\text { low-up }\end{array}$ \\
\hline $\begin{array}{l}\text { Selective reporting (re- } \\
\text { porting bias) }\end{array}$ & Unclear risk & No distinction between primary and secondary outcome \\
\hline Other bias & Unclear risk & The surgeon's skills were not described. \\
\hline
\end{tabular}

\section{Bruwer 2000}

Methods Randomised controlled trial


Bruwer 2000 (Continued)

Centres: one (South Africa)

Participants: premenopausal women with suspected acute appendicitis
Gender: $100 \%$ females
Age: 26 years (mean)
Histology: 15 of 34 participants had an inflamed appendix

Interventions

LAP: diagnostic laparoscopy followed by LA when acute appendicitis was visible or no other diagnosis was found; three-trocar technique, stump closure by catgut ligatures $(n=18)$

$\mathrm{OA}$ : open exploration either via right iliac fossa incision or lower midline laparotomy at the discretion of the operating surgeon. Appendix removed in all iliac fossa incisions $(n=16)$

Antibiotics: not mentioned

Outcomes

Rates of unnecessary appendectomies; hospital stay; return to full activities and work; complication rates; duration of operation; number of analgesic doses; analgesia duration

\section{Notes}

Follow-up: personal or telephonic interview 4 weeks postoperatively

No appendectomy: all appendices were resected

Declaration of interest: not reported

Funding: not reported

Preoperative imaging: not reported

Analgesia requirements: "Postoperatively, pethidine in a dose approximating $1 \mathrm{mg} / \mathrm{kg}$ was prescribed, and offered 6-hourly." No difference in the consumption of analgesia

Sample size calculation: not reported

\section{Risk of bias}

\section{Bias}

\section{Authors' judgement Support for judgement}

Random sequence genera- Low risk "computer-generated randomisation list"

tion (selection bias)

Allocation concealment Low risk "numbered envelopes containing the instruction open or laparoscopic"
(selection bias)

Blinding of participants Unclear risk Not reported
and personnel (perfor-

mance bias)

All outcomes

\begin{tabular}{lll}
\hline $\begin{array}{l}\text { Blinding of outcome as- } \\
\text { sessment (detection bias) } \\
\text { All outcomes }\end{array}$ & Unclear risk & Not reported \\
\hline $\begin{array}{l}\text { Incomplete outcome data } \\
\begin{array}{l}\text { (attrition bias) } \\
\text { All outcomes }\end{array}\end{array}$ & Low risk & $\begin{array}{l}\text { "Complete follow-up data to } 4 \text { weeks were available for all randomised pa- } \\
\text { tients." The single converted case was analysed according to ITT. }\end{array}$ \\
\hline $\begin{array}{l}\text { Selective reporting (re- } \\
\text { porting bias) }\end{array}$ & Unclear risk & No distinction between primary and secondary outcome \\
\hline Other bias & Low risk & Inexperienced surgical registrars were superceded as operators. \\
\hline
\end{tabular}


Cipe 2014

\begin{tabular}{ll}
\hline Methods & Randomised controlled trial \\
& Centres: one (Turkey) \\
\hline Participants & Participants: patients with appendicitis \\
& Gender: $44 \%$ females \\
& Age: 26.4 and 29.7 years (mean) in LA and OA \\
Histology: $90 \%$ inflamed & LA: three trocar technique: (10, 5, and 10 mm), stump closure with endo-loops ( $\mathrm{n}=126)$ \\
OA: via McBurney incision ( $\mathrm{n}=120)$ \\
Antibiotics: \\
- $\begin{array}{l}\text { Interventions } \\
\text { with complicated acute appendicitis: first generation cephalosporine and metronidazole (Flagyl, } 500 \\
\text { mg IV) according to clinical situation of each participant }\end{array}$
\end{tabular}

\section{Outcomes}

Pain scores (at 1, 6, 12, and 24 h; VAS); time of surgery; parenteral analgesics; length of hospital stay; postoperative complications (wound infection; pelvis abscess; atelectasis)

Notes Follow-up: at weekly intervals for three weeks

No appendectomy: not described

Declaration of interest: the authors had "no financial interest or conflict of interest to report"

Funding: not reported

Preoperative imaging: "Diagnosis of acute appendicitis was decided by the attending surgeon based on history, physical examination, laboratory and imaging techniques including ultrasonography or computed tomography."

Analgesia requirements: Diclofenac sodium $75 \mathrm{mg}$ IM (before extubation and during postoperative period as needed); the consumption of analgesia was significantly lower in the laparoscopic group.

Sample size calculation: partly reported

\section{Risk of bias}

\begin{tabular}{lll}
\hline Bias & Authors' judgement & Support for judgement \\
\hline $\begin{array}{l}\text { Random sequence genera- } \\
\text { tion (selection bias) }\end{array}$ & Low risk & "The patients were randomized ... via a computer-generated number." \\
\hline $\begin{array}{l}\text { Allocation concealment } \\
\text { (selection bias) }\end{array}$ & Unclear risk & Not reported \\
\hline $\begin{array}{l}\text { Blinding of participants } \\
\text { and personnel (perfor- } \\
\text { mance bias) }\end{array}$ & Unclear risk & Not reported \\
$\begin{array}{l}\text { All outcomes } \\
\text { Blinding of outcome as- } \\
\text { sessment (detection bias) } \\
\begin{array}{l}\text { All outcomes } \\
\hline\end{array}\end{array}$ & Unclear risk & Not reported \\
\hline
\end{tabular}


Cipe 2014 (Continued)

Incomplete outcome data Low risk "Patients with conversion to open approach were also excluded from the (attrition bias) study" ( $n=5)$.

All outcomes

In an email dated December 8th, 2014, the author provided data for all randomised participants to LA $(121+5)$ according to the ITT principle.
Selective reporting (re-
Unclear risk
No primary outcome measure was defined. porting bias)

\begin{tabular}{|c|c|c|}
\hline Other bias & Unclear risk & $\begin{array}{l}\text { "The consultant surgeons who were qualified to perform standardized LA and } \\
\text { OA or the residents under their supervision performed all operations ..." }\end{array}$ \\
\hline
\end{tabular}

\section{Cox 1996}

\begin{tabular}{ll}
\hline Methods & Randomised controlled trial \\
& Centres: one (Australia) \\
\hline Participants & Participants: men with a clinical diagnosis of acute appendicitis \\
& Gender: only men \\
Age: 25 years (median) in LA and OA \\
Histology: $88 \%$ inflamed
\end{tabular}

LA: three trocar technique, stump closure with endo loop $(\mathrm{n}=33)$
OA: via muscle splitting incision $(\mathrm{n}=31)$
Antibiotics: ceftriaxone $1 \mathrm{~g}$ IV and metronidazole 500mg IV
Outcomes Hospital stay; return to full activities; complication rates; duration of operation; number of doses of narcotic analgesia

Notes

\author{
Follow-up: "10 to 18 days after discharge" followed later by a telephone interview; 13 participants lost \\ to follow-up \\ No appendectomy: was done in one LA case with colitis, also analysed by ITT \\ Declaration of interest: not reported \\ Funding: not reported \\ Preoperative imaging: not reported
}

Analgesia requirements: "Postoperative analgesia was with intramuscular narcotic injection, oral analgesia, or both on a patient-request basis." "There was no significant difference in the mean number of parenteral narcotic doses required during the postoperative period."

Sample size calculation: not reported

\section{Risk of bias}

Bias Authors' judgement Support for judgement

Random sequence genera- Unclear risk
tion (selection bias)

tion (selection bias)

Allocation concealment $\quad$ Unclear risk Not reported
(selection bias)


Cox 1996 (Continued)

\begin{tabular}{|c|c|c|}
\hline $\begin{array}{l}\text { Blinding of participants } \\
\text { and personnel (perfor- } \\
\text { mance bias) } \\
\text { All outcomes }\end{array}$ & Unclear risk & Not reported \\
\hline $\begin{array}{l}\text { Blinding of outcome as- } \\
\text { sessment (detection bias) } \\
\text { All outcomes }\end{array}$ & Unclear risk & Not reported \\
\hline $\begin{array}{l}\text { Incomplete outcome data } \\
\text { (attrition bias) } \\
\text { All outcomes }\end{array}$ & Unclear risk & $\begin{array}{l}\text { "Thirteen patients ( } 7 \text { with open and } 6 \text { with laparoscopic procedures) were lost } \\
\text { to follow-up." Converted cases }(n=4) \text { and those receiving other surgery were } \\
\text { analysed by ITT. }\end{array}$ \\
\hline $\begin{array}{l}\text { Selective reporting (re- } \\
\text { porting bias) }\end{array}$ & Unclear risk & No distinction between primary and secondary outcome \\
\hline Other bias & Low risk & "Our study was performed after the initial learning phase of LA." \\
\hline
\end{tabular}

DeWilde 1991

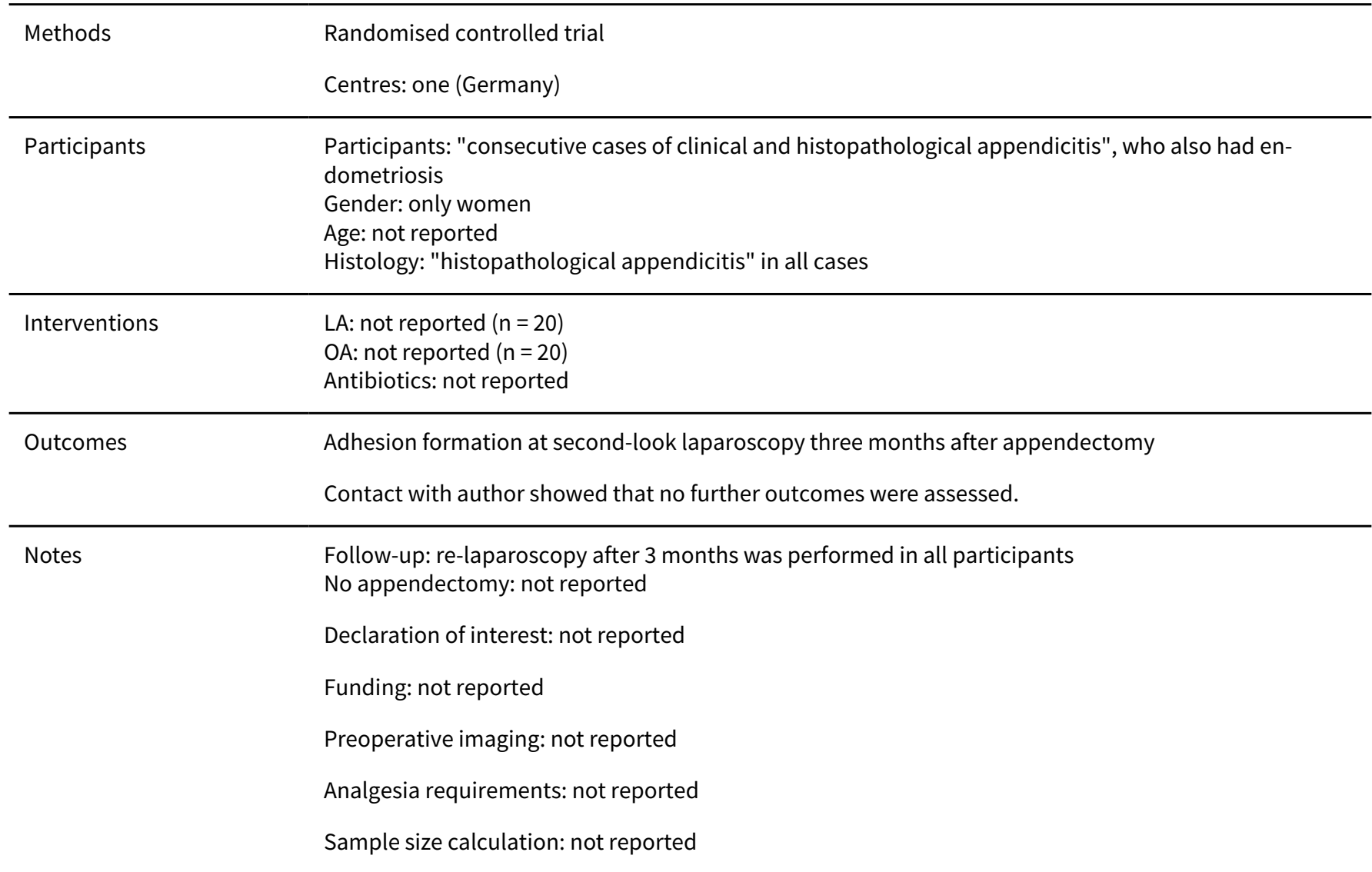

\section{Risk of bias}

\begin{tabular}{lll}
\hline Bias & Authors' judgement & Support for judgement \\
\hline $\begin{array}{l}\text { Random sequence genera- } \\
\text { tion (selection bias) }\end{array}$ & Unclear risk & Not reported \\
\hline
\end{tabular}


DeWilde 1991 (Continued)

\begin{tabular}{lll}
$\begin{array}{l}\text { Allocation concealment } \\
\text { (selection bias) }\end{array}$ & Unclear risk & Not reported \\
\hline $\begin{array}{l}\text { Blinding of participants } \\
\text { and personnel (perfor- } \\
\text { mance bias) }\end{array}$ & Unclear risk & Not reported \\
All outcomes & &
\end{tabular}

\begin{tabular}{lll}
\hline $\begin{array}{l}\text { Blinding of outcome as- } \\
\text { sessment (detection bias) } \\
\text { All outcomes }\end{array}$ & Unclear risk & Not reported \\
\hline $\begin{array}{l}\text { Incomplete outcome data } \\
\begin{array}{l}\text { (attrition bias) } \\
\text { All outcomes }\end{array}\end{array}$ & Unclear risk & $\begin{array}{l}\text { No reporting of the number and reasons for exclusions or completeness of fol- } \\
\text { low-up }\end{array}$ \\
\hline $\begin{array}{l}\text { Selective reporting (re- } \\
\text { porting bias) }\end{array}$ & Unclear risk & Insufficient information to permit judgement \\
\hline Other bias & Unclear risk & The surgeon's skills were not described. \\
\hline
\end{tabular}

\section{Eichen 1994}

\begin{tabular}{|c|c|}
\hline Methods & $\begin{array}{l}\text { Randomised controlled trial } \\
\text { Centres: one (Germany) }\end{array}$ \\
\hline Participants & $\begin{array}{l}\text { Participants: with a clinical diagnosis of chronic, acute, or perforated appendicitis } \\
\text { Gender: } 63 \% \text { females } \\
\text { Age: between } 8 \text { and } 75 \text { years } \\
\text { Histology: } 100 \% \text { inflamed (including chronic inflammation) }\end{array}$ \\
\hline Interventions & $\begin{array}{l}\text { LA: Access ports not described. Stump closure with Roeder loops }(n=97) \\
\text { OA: via muscle splitting incision, but without stump inversion }(n=96) \\
\text { Antibiotics: depending on intraoperative findings }\end{array}$ \\
\hline Outcomes & $\begin{array}{l}\text { Hospital stay; complication rates; duration of operation; pain intensity (VAS); analgesic medication; re- } \\
\text { turn to work }\end{array}$ \\
\hline \multirow[t]{6}{*}{ Notes } & $\begin{array}{l}\text { Follow-up: not reported } \\
\text { No appendectomy: not reported }\end{array}$ \\
\hline & Declaration of interest: not reported \\
\hline & Funding: not reported \\
\hline & Preoperative imaging: not reported \\
\hline & Analgesia requirements: not reported \\
\hline & Sample size calculation: not reported \\
\hline
\end{tabular}

\section{Risk of bias}


Eichen 1994 (Continued)
Random sequence genera- Low risk
In a letter (dated May 10,2004) one of the coauthors described that randomi- tion (selection bias) sation was done by "blindly" picking cards from an envelope containing 50 LA and $50 \mathrm{OA}$ lots.

\begin{tabular}{lll}
\hline $\begin{array}{l}\text { Allocation concealment } \\
\text { (selection bias) }\end{array}$ Low risk previous entry \\
\hline
\end{tabular}

Blinding of participants $\quad$ Unclear risk $\quad$ Not reported
and personnel (perfor-
mance bias)
All outcomes

\begin{tabular}{|c|c|c|}
\hline $\begin{array}{l}\text { Blinding of outcome as- } \\
\text { sessment (detection bias) } \\
\text { All outcomes }\end{array}$ & Unclear risk & Not reported \\
\hline $\begin{array}{l}\text { Incomplete outcome data } \\
\text { (attrition bias) } \\
\text { All outcomes }\end{array}$ & Unclear risk & $\begin{array}{l}\text { No reporting of the number and reasons for exclusions or completeness of fol- } \\
\text { low-up. The single converted case was analysed by ITT. }\end{array}$ \\
\hline $\begin{array}{l}\text { Selective reporting (re- } \\
\text { porting bias) }\end{array}$ & Unclear risk & No distinction between primary and secondary outcome \\
\hline Other bias & Low risk & The study authors mentioned their previous experience with 406 LA cases. \\
\hline
\end{tabular}

\section{Frazee 1994}

\begin{tabular}{|c|c|}
\hline Methods & $\begin{array}{l}\text { Randomised controlled trial } \\
\text { Centres: one (USA) }\end{array}$ \\
\hline Participants & $\begin{array}{l}\text { Participants: with "a preoperative diagnosis of acute appendicitis" } \\
\text { Gender: } 52 \% \text { females } \\
\text { Age: } 28 \text { and } 30 \text { years (mean) in LA and OA } \\
\text { Histology: } 82 \% \text { inflamed }\end{array}$ \\
\hline Interventions & $\begin{array}{l}\text { LA: three trocars }(10,10 \text {, and } 5 \mathrm{~mm}) \text {, stump closure with endo loops }(\mathrm{n}=38) \\
\text { OA: via muscle splitting incision }(\mathrm{n}=37) \\
\text { Antibiotics: not reported }\end{array}$ \\
\hline Outcomes & $\begin{array}{l}\text { Hospital stay; return to normal diet; return to full activities; complication rates; duration of operation; } \\
\text { duration of parental and oral analgesia, "informal cost analysis" }\end{array}$ \\
\hline \multirow[t]{6}{*}{ Notes } & $\begin{array}{l}\text { Follow-up: "by clinic visit or telephone interview at 2, 4, and } 8 \text { weeks postoperatively, and follow-up } \\
\text { was complete for all patients." } \\
\text { No appendectomy: not reported }\end{array}$ \\
\hline & Declaration of interest: not reported \\
\hline & Funding: not reported \\
\hline & Preoperative imaging: not reported \\
\hline & Analgesia requirements: not reported \\
\hline & Sample size calculation: not reported \\
\hline
\end{tabular}


Frazee 1994 (Continued)

Risk of bias

\begin{tabular}{|c|c|c|}
\hline Bias & Authors' judgement & Support for judgement \\
\hline $\begin{array}{l}\text { Random sequence genera- } \\
\text { tion (selection bias) }\end{array}$ & Unclear risk & "Patients were assigned randomly..." \\
\hline $\begin{array}{l}\text { Allocation concealment } \\
\text { (selection bias) }\end{array}$ & Unclear risk & Not reported \\
\hline $\begin{array}{l}\text { Blinding of participants } \\
\text { and personnel (perfor- } \\
\text { mance bias) } \\
\text { All outcomes }\end{array}$ & Unclear risk & Not reported \\
\hline $\begin{array}{l}\text { Blinding of outcome as- } \\
\text { sessment (detection bias) } \\
\text { All outcomes }\end{array}$ & Unclear risk & Not reported \\
\hline $\begin{array}{l}\text { Incomplete outcome data } \\
\text { (attrition bias) } \\
\text { All outcomes }\end{array}$ & Low risk & $\begin{array}{l}\text { "Follow-up was complete for all patients." Converted cases }(n=2,5 \%) \text { were } \\
\text { analysed by ITT. }\end{array}$ \\
\hline $\begin{array}{l}\text { Selective reporting (re- } \\
\text { porting bias) }\end{array}$ & Unclear risk & No distinction between primary and secondary outcome \\
\hline Other bias & High risk & $\begin{array}{l}\text { "Our study was initiated after } 2 \text { years of experience with LA." Still a learning } \\
\text { curve is likely, because "operating time continually dropped" during the study } \\
\text { period. }\end{array}$ \\
\hline
\end{tabular}

Goudar 2011

\begin{tabular}{ll} 
Methods & Randomised controlled trial \\
& Centre: one (India) \\
\hline Participants & Participants: "...age range of 12-48 years, with features which were suggestive of acute appendicitis..." \\
& Gender: $48 \%$ females \\
& Age: 23.9 years and 24.5 years (mean) in LA and OA \\
& Histology: three participants with negative appendicitis \\
& LA: three trocar technique (10, 5, and 5 mm); stump closure by endoloops ( $\mathrm{n}=114)$ \\
& OA: via McBurney or Lanz incision ( $\mathrm{n}=120)$ \\
Interventions & Antibiotics: not reported \\
\hline Outcomes & Time of surgery; pain (first three days, VAS); length of hospital stay; wound infection, intra-abdominal \\
& Follow-up: on the 8th day \\
No appendectomy: not reported & Declaration of interest: "No competing interest" \\
\hline Notes &
\end{tabular}


Funding: not reported

Preoperative imaging: "...ultrasound of the abdomen were routinely performed in all the cases."

Analgesia requirements: need for analgesics was not different between the two groups (details not reported)

Sample size calculation: not reported

\section{Risk of bias}

\begin{tabular}{|c|c|c|}
\hline Bias & Authors' judgement & Support for judgement \\
\hline $\begin{array}{l}\text { Random sequence genera- } \\
\text { tion (selection bias) }\end{array}$ & Unclear risk & "All patients were randomly divided into..." \\
\hline $\begin{array}{l}\text { Allocation concealment } \\
\text { (selection bias) }\end{array}$ & Unclear risk & Not reported \\
\hline $\begin{array}{l}\text { Blinding of participants } \\
\text { and personnel (perfor- } \\
\text { mance bias) } \\
\text { All outcomes }\end{array}$ & High risk & "This study was not blinded ..." \\
\hline $\begin{array}{l}\text { Blinding of outcome as- } \\
\text { sessment (detection bias) } \\
\text { All outcomes }\end{array}$ & High risk & "This study was not blinded ..." \\
\hline $\begin{array}{l}\text { Incomplete outcome data } \\
\text { (attrition bias) } \\
\text { All outcomes }\end{array}$ & High risk & $\begin{array}{l}\text { Conversions ( } n=6,5 \% \text { ) were excluded from the study, which violates the ITT } \\
\text { principle. }\end{array}$ \\
\hline $\begin{array}{l}\text { Selective reporting (re- } \\
\text { porting bias) }\end{array}$ & High risk & $\begin{array}{l}\text { No primary outcome measure was defined. Pain measured for the first three } \\
\text { consecutive days were not reported for the single days. }\end{array}$ \\
\hline Other bias & Unclear risk & The surgeon's skills were not described. \\
\hline
\end{tabular}

Gundavda 2012

\begin{tabular}{ll}
\hline Methods & Randomised controlled trial \\
& Centre: one (India) \\
\hline Participants & Participants: "...patients presenting with clinical diagnosis of appendicitis..." \\
& Gender: $53 \%$ females \\
& Age: 25.5 years and 25.6 years (mean) in LA and OA \\
& Histology: not reported \\
\hline LA: not reported ( $n=30)$ \\
OA: not reported ( $\mathrm{n}=30)$ \\
Antibiotics: not reported
\end{tabular}


Gundavda 2012 (Continued)

Outcomes Analgesic requirement; postoperative pain at rest (day 1, 3, 7, 14, 21, 28); return of bowel activities, starting of oral liquids; wound infections; scar size; length of hospital stay; full recovery; sick leave

Notes

Follow-up: 28 days

No appendectomy: not reported

Declaration of interest: "None declared"

Funding: "Nil"

Preoperative imaging: not reported

Analgesia requirements: "...diclofenac or tramadol most commonly on demand..." The number of injections was significantly lower in the laparoscopic group.

Sample size calculation: not reported

\section{Risk of bias}

\begin{tabular}{lll}
\hline Bias & Authors' judgement & Support for judgement \\
\hline $\begin{array}{l}\text { Random sequence genera- } \\
\text { tion (selection bias) }\end{array}$ & Unclear risk & $\begin{array}{l}\text { "In the present randomized clinical trial study... in which 60 patients were } \\
\text { equally distributed in equally in two treatment groups" }\end{array}$ \\
\hline $\begin{array}{l}\text { Allocation concealment } \\
\text { (selection bias) }\end{array}$ & Unclear risk & Not reported \\
\hline $\begin{array}{l}\text { Blinding of participants } \\
\text { and personnel (perfor- } \\
\text { mance bias) } \\
\text { All outcomes }\end{array}$ & Unclear risk & Not reported \\
\hline $\begin{array}{l}\text { Blinding of outcome as- } \\
\text { sessment (detection bias) }\end{array}$ & Unclear risk & Not reported \\
All outcomes & & \\
\hline $\begin{array}{l}\text { Incomplete outcome data } \\
\text { (attrition bias) } \\
\text { All outcomes }\end{array}$ & Unclear risk & There was no information on conversions, analyses or losses to follow-up. \\
\hline $\begin{array}{l}\text { Selective reporting (re- } \\
\text { porting bias) }\end{array}$ & Unclear risk & No distinction between primary and secondary outcomes \\
\hline \begin{tabular}{l} 
Other bias \\
\hline
\end{tabular} & Unclear risk & The surgeon's skills were not described. \\
\hline
\end{tabular}

\section{Hansen 1996}

\begin{tabular}{ll}
\hline Methods & Randomised controlled trial \\
& Centres: one (Australia) \\
\hline Participants & Participants: with "a diagnosis of acute appendicitis" \\
& Gender: $64 \%$ females \\
& Age: 25 and 22 years (median) in LA and OA \\
& Histology: $85 \%$ inflamed \\
\hline Interventions & LA: three (in some cases four) trocars, stump closure with a single endo loop $(n=86)$ \\
\hline
\end{tabular}

Laparoscopic versus open surgery for suspected appendicitis (Review) 
Hansen 1996 (Continued)

OA: via muscle splitting incision $(n=72)$

Antibiotics: cefoxitin $1 \mathrm{~g}$ IV

\begin{tabular}{ll}
\hline Outcomes & Hospital stay; return to normal bowel function; return to normal diet; return to full activities; complica- \\
tion rates; duration of operation; doses of analgesics
\end{tabular}

Notes Follow-up: at 1 and 4 weeks after surgery. Four participants were lost to follow-up (3\%)

No appendectomy: was performed in three laparoscopy cases

Declaration of interest: not reported

Funding: not reported

Preoperative imaging: not reported

Analgesia requirements: "After surgery the patients were prescribed intramuscular pethidine and oral panadeine." "The laparoscopic group required less narcotic analgesia $(P<0.0002)$..."

Sample size calculation: not reported

\section{Risk of bias}

\begin{tabular}{|c|c|c|}
\hline Bias & Authors' judgement & Support for judgement \\
\hline $\begin{array}{l}\text { Random sequence genera- } \\
\text { tion (selection bias) }\end{array}$ & Low risk & "random numbers table" \\
\hline $\begin{array}{l}\text { Allocation concealment } \\
\text { (selection bias) }\end{array}$ & Low risk & "sealed envelope system" \\
\hline $\begin{array}{l}\text { Blinding of participants } \\
\text { and personnel (perfor- } \\
\text { mance bias) } \\
\text { All outcomes }\end{array}$ & Unclear risk & Not reported \\
\hline $\begin{array}{l}\text { Blinding of outcome as- } \\
\text { sessment (detection bias) } \\
\text { All outcomes }\end{array}$ & Unclear risk & Not reported \\
\hline $\begin{array}{l}\text { Incomplete outcome data } \\
\text { (attrition bias) } \\
\text { All outcomes }\end{array}$ & High risk & $\begin{array}{l}\text { "4 patients could not be contacted after surgery, two in each group." Out of } \\
165 \text { participants, } 7 \text { were excluded after randomizations due to "protocol vi- } \\
\text { olations". Seven further participants who required conversion were also not } \\
\text { analysed according to ITT. }\end{array}$ \\
\hline $\begin{array}{l}\text { Selective reporting (re- } \\
\text { porting bias) }\end{array}$ & Unclear risk & No distinction between primary and secondary outcome \\
\hline Other bias & Low risk & Surgeons: with "a minimum of five laparoscopic appendectomies" \\
\hline
\end{tabular}

Hart 1996

\begin{tabular}{ll} 
Methods & Randomised controlled trial \\
& Centres: one (United Kingdom) \\
\hline Participants & Participants: with "a clinical diagnosis of acute appendicitis" \\
& Gender: $38 \%$ females \\
& Age: 29.4 and 32.6 years (mean) in LA and OA \\
\hline
\end{tabular}


Hart 1996 (Continued)

Histology: $75 \%$ inflamed

LA: four trocars $(10,10,5$, and $5 \mathrm{~mm})$, stump closure with two endoloops $(\mathrm{n}=44)$
OA: via muscle splitting incision $(\mathrm{n}=37)$
Antibiotics: ceftizoxime $1 \mathrm{~g}$ IV

Outcomes Hospital stay; return to full activities; complication rates; duration of operation; doses of analgesics;
pain (VAS)

Notes $\quad$ Follow-up: "in the clinic at 7 and 28 days after discharge"

No appendectomy: "All appendices were removed regardless of gross appearance."

Declaration of interest: not reported

Funding: not reported

Preoperative imaging: not reported

Analgesia requirements: "Our patients required significantly $(P<0.001)$ fewer postoperative narcotic injections after LA than after OA..."

Sample size calculation: not reported

\section{Risk of bias}

\begin{tabular}{|c|c|c|}
\hline Bias & Authors' judgement & Support for judgement \\
\hline $\begin{array}{l}\text { Random sequence genera- } \\
\text { tion (selection bias) }\end{array}$ & Low risk & "by opening computer-generated randomized..." \\
\hline $\begin{array}{l}\text { Allocation concealment } \\
\text { (selection bias) }\end{array}$ & Low risk & "...sealed envelopes" \\
\hline $\begin{array}{l}\text { Blinding of participants } \\
\text { and personnel (perfor- } \\
\text { mance bias) } \\
\text { All outcomes }\end{array}$ & Unclear risk & Not reported \\
\hline $\begin{array}{l}\text { Blinding of outcome as- } \\
\text { sessment (detection bias) } \\
\text { All outcomes }\end{array}$ & Unclear risk & Not reported \\
\hline $\begin{array}{l}\text { Incomplete outcome data } \\
\text { (attrition bias) } \\
\text { All outcomes }\end{array}$ & High risk & $\begin{array}{l}\text { No reporting of the number and reasons for exclusions or completeness of fol- } \\
\text { low-up. After conversion from LA to OA, four participants ( } 9 \% \text { of laparoscopy } \\
\text { group) were excluded from the analysis, which clearly violates the ITT princi- } \\
\text { ple. }\end{array}$ \\
\hline $\begin{array}{l}\text { Selective reporting (re- } \\
\text { porting bias) }\end{array}$ & Low risk & $\begin{array}{l}\text { Primary outcomes (number of days in hospital and time to full recovery) were } \\
\text { described. }\end{array}$ \\
\hline Other bias & Low risk & Surgeons were trained in the techniques of LA. \\
\hline
\end{tabular}

Hebebrand 1994

$\begin{array}{ll}\text { Methods } & \text { Randomised controlled trial } \\ \text { Centres: one (Germany) }\end{array}$


Hebebrand 1994 (Continued)

Participants

Participants: with a acute abdominal pain and a presumptive diagnosis of appendicitis

Gender: $53 \%$ females

Age: 23 and 24 years (median, excluding converted cases) in LA and OA

Histology: $91 \%$ inflamed
LA: three trocar technique $(10,10$, and $5 \mathrm{~mm})$, stump closure with three endo loops $(n=25)$

OA: via muscle splitting incision $(n=23)$

Antibiotics: not reported

Outcomes

Pain (VAS) during bed rest, while standing, and while moving; consumption of analgesics; fatigue (VAS); complications; hospital stay; return to normal bowel function; duration of operation; cosmesis (VAS)

Follow-up: until hospital discharge
No appendectomy: none
Declaration of interest: not reported
Funding: not reported
Preoperative imaging: ultrasound scan
Analgesia requirements: ("After surgery the patients were prescribed tramadol 400 mg and metami-
zol $5 g$ in 500 ml NaCl..., ibuprofen as needed"; consumption of analgesia was lower in the laparoscopic
group...)
Sample size calculation: adequately reported

\section{Risk of bias}

\begin{tabular}{|c|c|c|}
\hline Bias & Authors' judgement & Support for judgement \\
\hline $\begin{array}{l}\text { Random sequence genera- } \\
\text { tion (selection bias) }\end{array}$ & Unclear risk & "balanced block randomisation" \\
\hline $\begin{array}{l}\text { Allocation concealment } \\
\text { (selection bias) }\end{array}$ & Low risk & Personal contact with trialists revealed that sealed envelopes were used \\
\hline $\begin{array}{l}\text { Blinding of participants } \\
\text { and personnel (perfor- } \\
\text { mance bias) } \\
\text { All outcomes }\end{array}$ & High risk & None \\
\hline $\begin{array}{l}\text { Blinding of outcome as- } \\
\text { sessment (detection bias) } \\
\text { All outcomes }\end{array}$ & High risk & None \\
\hline $\begin{array}{l}\text { Incomplete outcome data } \\
\text { (attrition bias) } \\
\text { All outcomes }\end{array}$ & Low risk & $\begin{array}{l}\text { The results for converted cases ( } n=9,26 \% \text { ) were given separately, so ITT } \\
\text { analysis was possible }\end{array}$ \\
\hline $\begin{array}{l}\text { Selective reporting (re- } \\
\text { porting bias) }\end{array}$ & Low risk & $\begin{array}{l}\text { Primary outcome (pain during bed rest, while standing, and while moving) was } \\
\text { described. }\end{array}$ \\
\hline Other bias & High risk & "Still a learning curve is present to a certain degree." \\
\hline
\end{tabular}


Heikkinen 1998

\begin{tabular}{|c|c|}
\hline Methods & $\begin{array}{l}\text { Randomised controlled trial } \\
\text { Centres: one (Finland) }\end{array}$ \\
\hline Participants & $\begin{array}{l}\text { Participants: "with suspected appendicitis". Those with suspected perforation were excluded. } \\
\text { Gender: } 48 \% \text { females } \\
\text { Age: } 34 \text { and } 37 \text { years (median) in LA and OA } \\
\text { Histology: } 85 \% \text { inflamed }\end{array}$ \\
\hline Interventions & $\begin{array}{l}\text { LAP: diagnostic laparoscopy, then LA; three trocars }(10,13 \text {, and } 5 \mathrm{~mm})(\mathrm{n}=19) \\
\text { OA: via muscle splitting incision }(\mathrm{n}=21) \\
\text { Antibiotics: metronidazole } 500 \mathrm{mg} \text { IV }\end{array}$ \\
\hline Outcomes & $\begin{array}{l}\text { Hospital stay; return to full activities and work; complication rates; duration of operation; pain scores } \\
\text { (VAS), fatigue; participant satisfaction; costs (direct and indirect) }\end{array}$ \\
\hline \multirow[t]{6}{*}{ Notes } & $\begin{array}{l}\text { Follow-up: participants were asked to return questionnaires } 1 \text { to } 2 \text { months after the operation. } \\
\text { No appendectomy: was necessary in one LA participant, analysed by ITT }\end{array}$ \\
\hline & Declaration of interest: not reported \\
\hline & Funding: not reported \\
\hline & Preoperative imaging: not reported \\
\hline & $\begin{array}{l}\text { Analgesia requirements: "Postoperative pain medication at the hospital consisted of oxycodone-chlo- } \\
\text { ride and ketoprofen. Ketoprofen (100-mg capsules) was described for use at home." "The postoperative } \\
\text { need for analgesia ... was similar for both groups." }\end{array}$ \\
\hline & Sample size calculation: not reported \\
\hline
\end{tabular}

\section{Risk of bias}

\begin{tabular}{lll}
\hline Bias & Authors' judgement & Support for judgement \\
\hline $\begin{array}{l}\text { Random sequence genera- } \\
\text { tion (selection bias) }\end{array}$ & Unclear risk & "The randomisation was done..." \\
\hline $\begin{array}{l}\text { Allocation concealment } \\
\text { (selection bias) }\end{array}$ & Low risk & "...by opening a sealed envelope" \\
\hline $\begin{array}{l}\text { Blinding of participants } \\
\text { and personnel (perfor- } \\
\text { mance bias) }\end{array}$ & Unclear risk & Not reported \\
All outcomes & \\
\hline
\end{tabular}

\begin{tabular}{|c|c|c|}
\hline $\begin{array}{l}\text { Blinding of outcome as- } \\
\text { sessment (detection bias) } \\
\text { All outcomes }\end{array}$ & Unclear risk & Not reported \\
\hline $\begin{array}{l}\text { Incomplete outcome data } \\
\text { (attrition bias) } \\
\text { All outcomes }\end{array}$ & Unclear risk & $\begin{array}{l}\text { Completeness of follow-up was not reported in detail. Conversion from LA to } \\
\text { OA was necessary in one participant, whose results were reported separately. } \\
\text { This allowed for ITT analysis. }\end{array}$ \\
\hline
\end{tabular}

\begin{tabular}{lll}
\hline $\begin{array}{l}\text { Selective reporting (re- } \\
\text { porting bias) }\end{array}$ & Unclear risk & No distinction between primary and secondary outcome \\
\hline Other bias & Low risk & $\begin{array}{l}\text { Trials surgeons had "substantial experience in laparoscopic and open appen- } \\
\text { dectomies." }\end{array}$ \\
\hline
\end{tabular}


Hellberg 1999

\begin{tabular}{|c|c|}
\hline Methods & $\begin{array}{l}\text { Randomised controlled trial } \\
\text { Centres: one university hospital and four county hospitals (Sweden) }\end{array}$ \\
\hline Participants & $\begin{array}{l}\text { Participants: "with suspected acute appendicitis" } \\
\text { Gender: not reported } \\
\text { Age: not reported } \\
\text { Histology: } 80 \% \text { inflamed }\end{array}$ \\
\hline Interventions & $\begin{array}{l}\text { LA: usually three trocars }(11,11 \text {, and } 5 \mathrm{~mm}) \text {; stump closure by different techniques }(\mathrm{n}=244) \\
\text { OA: through a McBurney incision }(\mathrm{n}=256) \\
\text { Antibiotics: cefuroxime } 1.5 \mathrm{~g} \text { and metronidazole } 500 \mathrm{mg} \mathrm{IV}\end{array}$ \\
\hline Outcomes & $\begin{array}{l}\text { Hospital stay; return to full activities and work; complication rates; duration of operation; anaesthesia } \\
\text { time; pain scores on days 1, 7, and } 14 \text { (VAS), functional index (combined from three tests) }\end{array}$ \\
\hline Notes & $\begin{array}{l}\text { Follow-up: weekly, usually until } 28 \text { days postoperatively. All } 500 \text { participants "were followed until full } \\
\text { recovery." } \\
\text { No appendectomy: } \mathrm{n}=8 \text {, in whom other surgical disease was found. These cases were excluded as } \\
\text { withdrawals. } \\
\text { Declaration of interest: not reported } \\
\text { Funding: not reported } \\
\text { Preoperative imaging: not reported } \\
\text { Analgesia requirements: not reported } \\
\text { Sample size calculation: adequately reported }\end{array}$ \\
\hline
\end{tabular}

\section{Risk of bias}

\begin{tabular}{|c|c|c|}
\hline Bias & Authors' judgement & Support for judgement \\
\hline $\begin{array}{l}\text { Random sequence genera- } \\
\text { tion (selection bias) }\end{array}$ & Low risk & "Randomization was in blocks of four, computer-generated..." \\
\hline $\begin{array}{l}\text { Allocation concealment } \\
\text { (selection bias) }\end{array}$ & Low risk & "using numbered closed envelopes drawn in sequential order." \\
\hline $\begin{array}{l}\text { Blinding of participants } \\
\text { and personnel (perfor- } \\
\text { mance bias) } \\
\text { All outcomes }\end{array}$ & Unclear risk & Not reported \\
\hline $\begin{array}{l}\text { Blinding of outcome as- } \\
\text { sessment (detection bias) } \\
\text { All outcomes }\end{array}$ & Unclear risk & Not reported \\
\hline $\begin{array}{l}\text { Incomplete outcome data } \\
\text { (attrition bias) } \\
\text { All outcomes }\end{array}$ & High risk & $\begin{array}{l}\text { The remaining } 500 \text { participants were followed until full recovery. Converted } \\
\text { cases ( } \mathrm{n}=30,12 \%) \text { were analysed by ITT, but those receiving other surgery } \\
\text { were excluded from analysis. In total, there were } 23 \text { withdrawals, who met pre- } \\
\text { specified criteria. }\end{array}$ \\
\hline $\begin{array}{l}\text { Selective reporting (re- } \\
\text { porting bias) }\end{array}$ & Unclear risk & No distinction between primary and secondary outcome \\
\hline
\end{tabular}


Hellberg 1999 (Continued)

Other bias Low risk Surgeons were required to have an experience of more than 10 LAs.

Helmy 2001

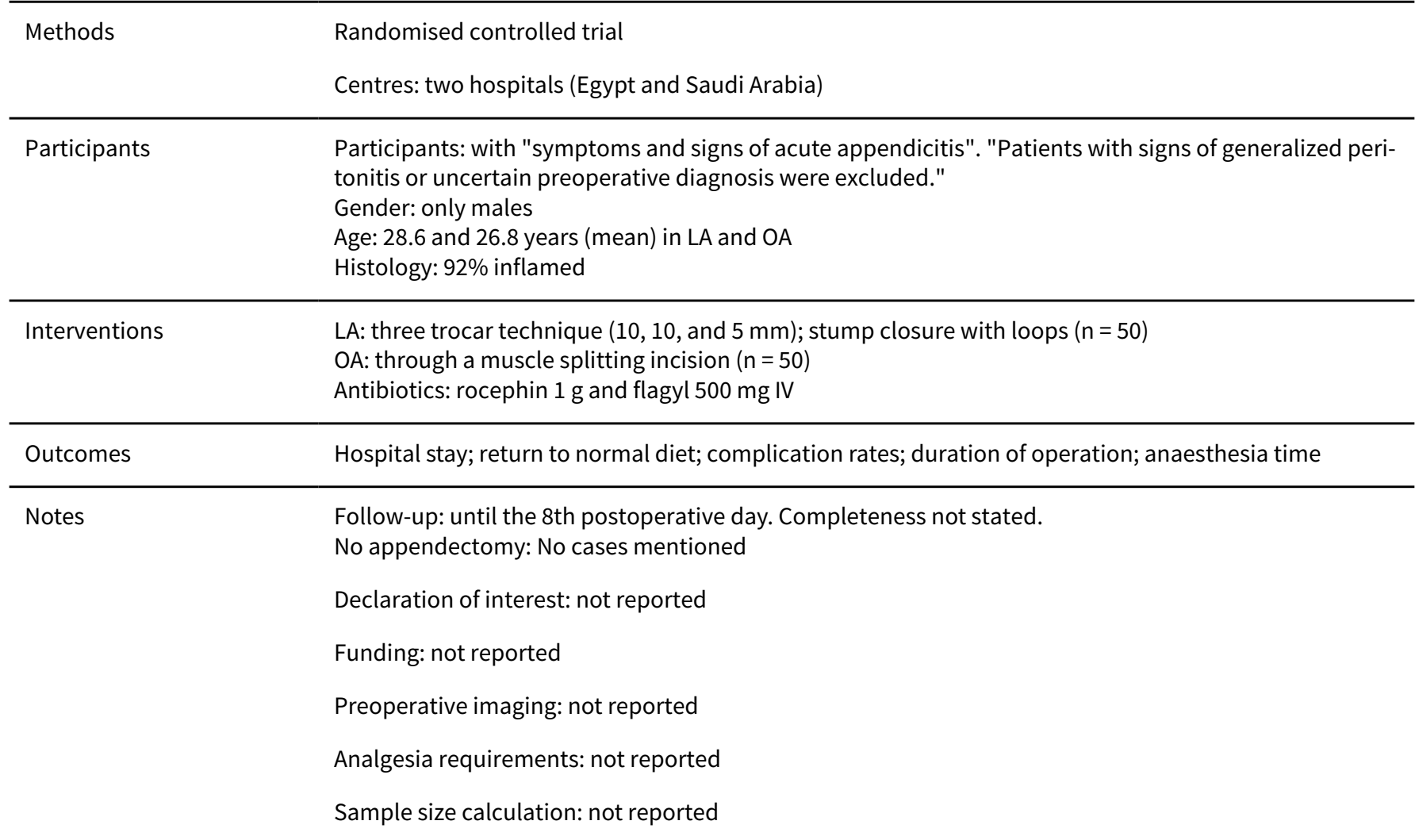

\section{Risk of bias}

\begin{tabular}{|c|c|c|}
\hline Bias & Authors' judgement & Support for judgement \\
\hline $\begin{array}{l}\text { Random sequence genera- } \\
\text { tion (selection bias) }\end{array}$ & Unclear risk & "were randomized" \\
\hline $\begin{array}{l}\text { Allocation concealment } \\
\text { (selection bias) }\end{array}$ & Unclear risk & Not reported \\
\hline $\begin{array}{l}\text { Blinding of participants } \\
\text { and personnel (perfor- } \\
\text { mance bias) } \\
\text { All outcomes }\end{array}$ & Unclear risk & Not reported \\
\hline $\begin{array}{l}\text { Blinding of outcome as- } \\
\text { sessment (detection bias) } \\
\text { All outcomes }\end{array}$ & Unclear risk & Not reported \\
\hline $\begin{array}{l}\text { Incomplete outcome data } \\
\text { (attrition bias) } \\
\text { All outcomes }\end{array}$ & Unclear risk & $\begin{array}{l}\text { No reporting of the number and reasons for exclusions or completeness of fol- } \\
\text { low-up. Converted cases }(n=9,18 \%) \text { were apparently analysed by ITT. }\end{array}$ \\
\hline
\end{tabular}


Helmy 2001 (Continued)

Selective reporting (re- $\quad$ Unclear risk $\quad$ No distinction between primary and secondary outcome
porting bias)

Other bias Low risk Surgeons were described as "skilled".

Henle 1996

\begin{tabular}{ll}
\hline Methods & Randomised controlled trial \\
Centres: not reported (Austria)
\end{tabular}

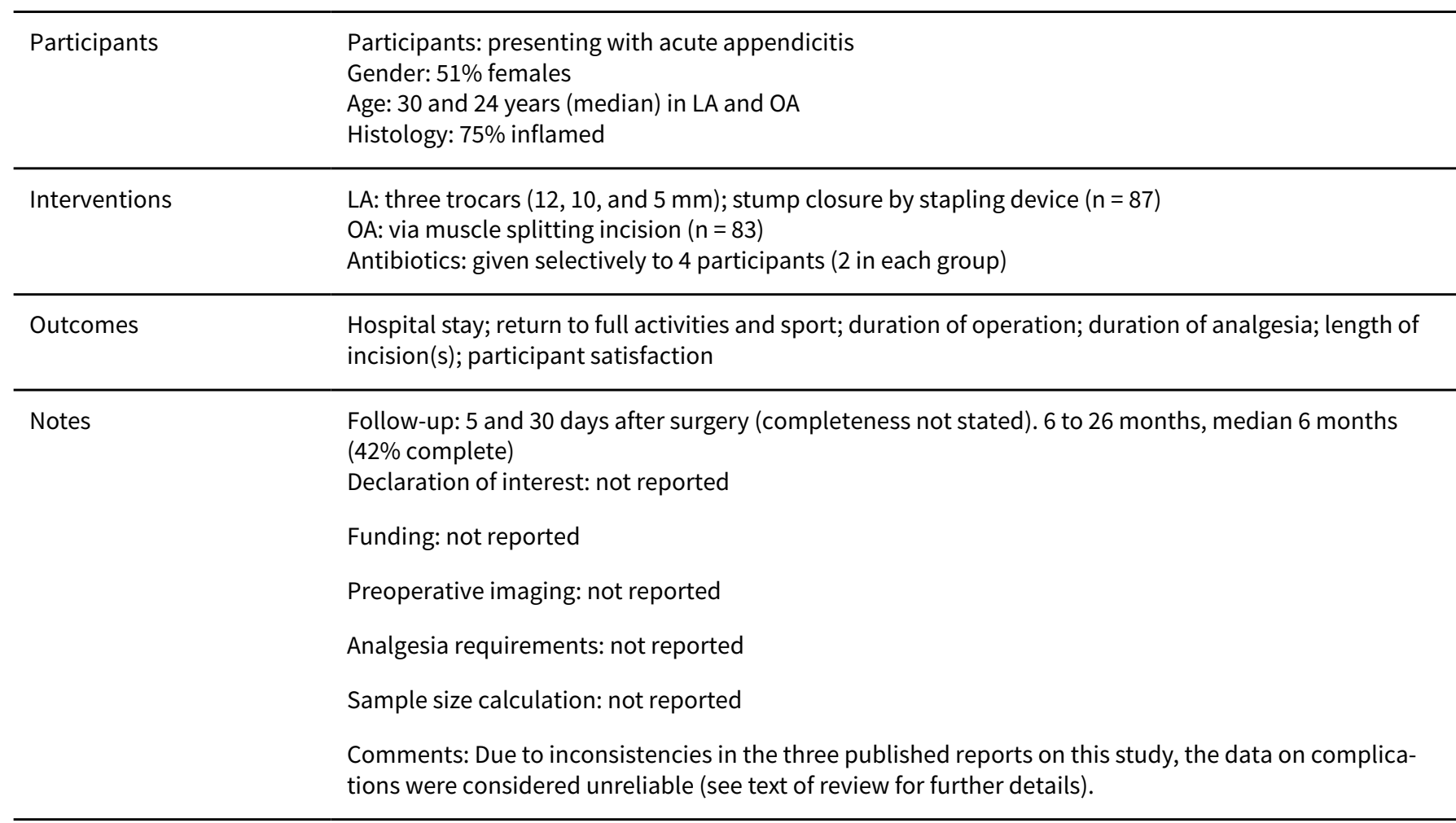

\section{Risk of bias}

Bias Authors' judgement Support for judgement

Random sequence genera- Low risk "using a table of random numbers."

tion (selection bias)

\begin{tabular}{lll}
\hline $\begin{array}{l}\text { Allocation concealment } \\
\text { (selection bias) }\end{array}$ & Unclear risk & Not reported \\
\hline $\begin{array}{l}\text { Blinding of participants } \\
\text { and personnel (perfor- } \\
\text { mance bias) }\end{array}$ & Unclear risk & Not reported \\
$\begin{array}{ll}\text { All outcomes } \\
\text { Blinding of outcome as- } \\
\text { sessment (detection bias) } \\
\text { All outcomes }\end{array}$ & Unclear risk & Not reported \\
\hline
\end{tabular}


Henle 1996 (Continued)

Incomplete outcome data High risk No reporting of losses of follow-up. One participant was excluded from all (attrition bias) analyses after conversion from OA to LA. Thus, the study was not analysed ITT.

All outcomes

\begin{tabular}{lll}
$\begin{array}{l}\text { Selective reporting (re- } \\
\text { porting bias) }\end{array}$ & Unclear risk & No distinction between primary and secondary outcome \\
\hline Other bias & High risk & $\begin{array}{l}\text { A surgical learning curve must be assumed, as } 60 \% \text { of surgical complications } \\
\text { after LA occurred in the first } 18 \text { participants (21\%). }\end{array}$
\end{tabular}

\section{Huang 2001}

\begin{tabular}{|c|c|}
\hline Methods & $\begin{array}{l}\text { Randomised controlled trial } \\
\text { Centres: one (Taiwan) }\end{array}$ \\
\hline Participants & $\begin{array}{l}\text { Participants: those in whom "appendicitis remained as the most likely diagnosis". Cases with local ab- } \\
\text { scess or peritonitis were excluded. } \\
\text { Gender: } 45 \% \text { ( } 34 \text { of } 75) \text { females } \\
\text { Age: } 32.2,23.2 \text { and } 33.8 \text { years (mean) in normal LA, needlescopic LA and OA } \\
\text { Histology: } 88 \% \text { (79/90) inflamed }\end{array}$ \\
\hline Interventions & $\begin{array}{l}\text { LAP: diagnostic laparoscopy, then LA, either with normal instruments }(10,5 \text {, and } 5 \mathrm{~mm})(\mathrm{n}=23) \text { or } \\
\text { needlescopic instruments }(10,2 \text {, and } 2 \mathrm{~mm})(\mathrm{n}=26) \text {; stump closure by variable techniques } \\
\text { OA: via } 4-5 \mathrm{~cm} \text { muscle-splitting incision; no purse-string suture }(\mathrm{n}=26) \\
\text { Antibiotics: } 1 \text { g cefamezine IV }\end{array}$ \\
\hline Outcomes & $\begin{array}{l}\text { Hospital stay; complication rates; duration of operation; negative appendectomy rate; analgesic use; } \\
\text { return to normal diet }\end{array}$ \\
\hline Notes & $\begin{array}{l}\text { Follow-up: one week } \\
\text { Conversions: "There was no conversion to an open procedure". In } 3 \text { participants, however, who were } \\
\text { randomised to laparoscopy, a decision to convert was made preoperatively. } \\
\text { No appendectomy: was performed in } 6 \text { cases, which were not reported on in the article. } \\
\text { Declaration of interest: not reported } \\
\text { Funding: not reported } \\
\text { Preoperative imaging: not reported } \\
\text { Analgesia requirements: not reported } \\
\text { Sample size calculation: not reported }\end{array}$ \\
\hline
\end{tabular}

\section{Risk of bias}

\begin{tabular}{lll}
\hline Bias & Authors' judgement & Support for judgement \\
\hline $\begin{array}{l}\text { Random sequence genera- } \\
\text { tion (selection bias) }\end{array}$ & Unclear risk & "by random selection" \\
\hline $\begin{array}{l}\text { Allocation concealment } \\
\text { (selection bias) }\end{array}$ & Low risk & "sealed envelopes" \\
\hline $\begin{array}{l}\text { Blinding of participants } \\
\begin{array}{l}\text { and personnel (perfor- } \\
\text { mance bias) }\end{array}\end{array}$ & Unclear risk & Not reported \\
\hline
\end{tabular}


Huang 2001 (Continued)

All outcomes

\begin{tabular}{|c|c|c|}
\hline $\begin{array}{l}\text { Blinding of outcome as- } \\
\text { sessment (detection bias) } \\
\text { All outcomes }\end{array}$ & Low risk & $\begin{array}{l}\text { The ward nurses, who measured clinical recovery and decided on discharge } \\
\text { were blinded since wounds were covered with large bandages by a separate } \\
\text { person. }\end{array}$ \\
\hline
\end{tabular}

Incomplete outcome data Unclear risk (attrition bias)

Follow-up was apparently complete. The study reported on 75 participants af-

All outcomes ter several post hoc exclusions. The authors informed us that initially 90 participants were randomised equally into the 3 groups and provided complication results for these excluded cases, thus partially allowing for ITT analysis.

\begin{tabular}{lll}
\hline $\begin{array}{l}\text { Selective reporting (re- } \\
\text { porting bias) }\end{array}$ & Unclear risk & No distinction between primary and secondary outcome \\
\hline Other bias & Low risk & More than 2 years of experience in laparoscopy was required for trial surgeons. \\
\hline
\end{tabular}

Ignacio 2003

$\begin{array}{ll}\text { Methods } & \text { Randomised controlled trial } \\ \text { Centres: one naval medical centre (USA) }\end{array}$

\begin{tabular}{ll}
\hline Participants & Participants: "men presenting with signs and symptoms of acute appendicitis" \\
& Gender: men only \\
& Age: 28.4 and 27.4 years (mean) in LA and OA \\
Histology: $90 \%$ inflamed
\end{tabular}

LA: three trocar technique; stump closure apparently by different techniques $(n=26)$
OA: via muscle splitting incision $(n=26)$
Antibiotics: were given to all participants, but drug not specified

\begin{tabular}{|c|c|}
\hline Outcomes & $\begin{array}{l}\text { Hospital stay; return to work; complication rates; duration of operation; pain intensity (VAS); costs of } \\
\text { operation }\end{array}$ \\
\hline \multirow[t]{6}{*}{ Notes } & $\begin{array}{l}\text { Follow-up: "at weekly intervals" apparently until return to work } \\
\text { No appendectomy: not reported }\end{array}$ \\
\hline & Declaration of interest: not reported \\
\hline & $\begin{array}{l}\text { Funding: "The Chief; Navy Bureau of Medicine and Surgery, Washington DC, Clinical investigation Pro- } \\
\text { gram sponsored this study (CIP \#P00-058)." }\end{array}$ \\
\hline & Preoperative imaging: not reported \\
\hline & Analgesia requirements: not reported \\
\hline & Sample size calculation: partly reported \\
\hline
\end{tabular}

\section{Risk of bias}

\begin{tabular}{lll}
\hline Bias & Authors' judgement & Support for judgement \\
\hline $\begin{array}{l}\text { Random sequence genera- } \\
\text { tion (selection bias) }\end{array}$ & Low risk & $\begin{array}{l}\text { In an email dated September 5th, 2007, the author added that "block-random- } \\
\text { ized list was computer generated..." }\end{array}$ \\
\hline $\begin{array}{l}\text { Allocation concealment } \\
\text { (selection bias) }\end{array}$ & Low risk & "...with sealed envelopes." \\
\hline
\end{tabular}


Ignacio 2003 (Continued)

Blinding of participants Low risk_ Participants and postoperative care providers were blinded and personnel (performance bias)

All outcomes

\begin{tabular}{|c|c|c|}
\hline $\begin{array}{l}\text { Blinding of outcome as- } \\
\text { sessment (detection bias) } \\
\text { All outcomes }\end{array}$ & Unclear risk & Not reported \\
\hline $\begin{array}{l}\text { Incomplete outcome data } \\
\text { (attrition bias) } \\
\text { All outcomes }\end{array}$ & Low risk & $\begin{array}{l}\text { Apparently no exclusions or losses to follow-up. The single converted case was } \\
\text { analysed according to ITT. }\end{array}$ \\
\hline $\begin{array}{l}\text { Selective reporting (re- } \\
\text { porting bias) }\end{array}$ & Unclear risk & No distinction between primary and secondary outcome \\
\hline Other bias & Low risk & $\begin{array}{l}\text { Surgeons had to have "experience of more than } 15 \text { open and laparoscopic ap- } \\
\text { pendectomies." }\end{array}$ \\
\hline
\end{tabular}

\section{Jadallah 1994}

\begin{tabular}{|c|c|}
\hline Methods & $\begin{array}{l}\text { Randomised controlled trial } \\
\text { Centres: one (Kuwait) }\end{array}$ \\
\hline Participants & $\begin{array}{l}\text { Participants: women of childbearing age with acute lower abdominal pain suggestive of acute appen- } \\
\text { dicitis } \\
\text { Gender: women only } \\
\text { Age: not reported }\end{array}$ \\
\hline & Histology: $58 \%$ inflamed \\
\hline
\end{tabular}

Interventions

LAP: Diagnostic laparoscopy followed by open appendectomy when acute appendicitis was visible or no other diagnosis was found $(n=20+30)$

OPEN: described only as "conventional laparotomy" $(n=50)$

Antibiotics: $80 \mathrm{mg}$ gentamicin and $500 \mathrm{mg}$ metronidazole IV

Outcomes Rates of unnecessary appendectomies and participants without diagnosis established; complications; duration of surgery; hospital stay

Notes

Follow-up: "at least two weeks after discharge"

No appendectomy: was needed in 19 (38\%) participants, who were reported on separately.

Declaration of interest: not reported

Funding: not reported

Preoperative imaging: not reported

Analgesia requirements: not reported

Sample size calculation: not reported

\section{Risk of bias}


Jadallah 1994 (Continued)

Random sequence genera- Unclear risk "The patients were randomly allocated to two groups..." tion (selection bias)

\begin{tabular}{lll}
\hline $\begin{array}{l}\text { Allocation concealment } \\
\text { (selection bias) }\end{array}$ & Low risk & \\
\hline $\begin{array}{l}\text { Blinding of participants opening a sealed envelope" } \\
\text { and personnel (perfor- }\end{array}$ & Unclear risk & Not reported \\
mance bias) & & \\
All outcomes &
\end{tabular}

\begin{tabular}{lll}
\hline $\begin{array}{l}\text { Blinding of outcome as- } \\
\text { sessment (detection bias) } \\
\text { All outcomes }\end{array}$ & Unclear risk & Not reported \\
\hline $\begin{array}{l}\text { Incomplete outcome data } \\
\text { (attrition bias) }\end{array}$ & Low risk & $\begin{array}{l}\text { No reporting of the number and reasons for exclusions or completeness of fol- } \\
\text { low-up. Data were reported separately for participants with laparoscopy on- } \\
\text { ly and those with LA and OA, but pooled estimates could be calculated for the } \\
\text { complete group by ITT analysis. }\end{array}$ \\
\hline
\end{tabular}

\begin{tabular}{lll}
$\begin{array}{l}\text { Selective reporting (re- } \\
\text { porting bias) }\end{array}$ & Unclear risk & No distinction between primary and secondary outcome. \\
\hline Other bias & Unclear risk & The surgeon's skills are not described. \\
\hline
\end{tabular}

Kaiser 2006

\begin{tabular}{|c|c|}
\hline Methods & $\begin{array}{l}\text { Randomised controlled trial } \\
\text { Centers: one (Germany) }\end{array}$ \\
\hline Participants & $\begin{array}{l}\text { Participants: with a clinical diagnosis of acute appendicitis } \\
\text { Gender: } 66 \% \text { females } \\
\text { Age: only distribution into age groups reported } \\
\text { Histology: } 56 \% \text { inflamed (38\% no inflammation, } 6 \% \text { missing) }\end{array}$ \\
\hline Interventions & $\begin{array}{l}\text { LA: three trocar technique }(12,10 \text {, and } 5 \mathrm{~mm} \text { ); stump closure by EndoGIA (in > } 95 \% \text { of cases) }(n=61) \\
\text { OA: via muscle splitting incision }(n=75) \\
\text { Antibiotics: selected participants ( } 13 \mathrm{LA} \text { and } 20 \text { OA cases) received antibiotics at the induction of } \\
\text { anaesthesia (described on page } 27) \text {. In most cases, antibiotic therapy was continued postoperatively. }\end{array}$ \\
\hline Outcomes & $\begin{array}{l}\text { Hospital stay; return to full activities; return to normal diet; complication rates; duration of operation; } \\
\text { participant satisfaction; cosmesis; various laboratory and immunological parameters }\end{array}$ \\
\hline \multirow[t]{6}{*}{ Notes } & $\begin{array}{l}\text { Follow-up: until hospital discharge. All participants received a postal questionnaire to elicit subjective } \\
\text { outcomes, but only } 33 \text { of } 136 \text { participants replied. } \\
\text { No appendectomy: not reported }\end{array}$ \\
\hline & Declaration of interest: not reported \\
\hline & Funding: not reported \\
\hline & Preoperative imaging: not reported \\
\hline & Analgesia requirements: not reported \\
\hline & Sample size calculation: not reported \\
\hline
\end{tabular}


Kaiser 2006 (Continued)

Risk of bias

\begin{tabular}{|c|c|c|}
\hline Bias & Authors' judgement & Support for judgement \\
\hline $\begin{array}{l}\text { Random sequence genera- } \\
\text { tion (selection bias) }\end{array}$ & Unclear risk & $\begin{array}{l}\text { A block randomisation was described, but no details were provided on the } \\
\text { generation of the sequence. Randomisation was stratified for weight, gender, } \\
\text { and age (all as dichotomous variables). }\end{array}$ \\
\hline $\begin{array}{l}\text { Allocation concealment } \\
\text { (selection bias) }\end{array}$ & Low risk & $\begin{array}{l}\text { "Randomisation aimed at achieving structural similarity between groups and } \\
\text { was done by using sealed envelopes." ("Die Randomisierung mit dem Ziel der } \\
\text { Strukturgleichheit erfolgte mit verschlossenen Briefumschlägen.") }\end{array}$ \\
\hline $\begin{array}{l}\text { Blinding of participants } \\
\text { and personnel (perfor- } \\
\text { mance bias) } \\
\text { All outcomes }\end{array}$ & Unclear risk & Not reported \\
\hline $\begin{array}{l}\text { Blinding of outcome as- } \\
\text { sessment (detection bias) } \\
\text { All outcomes }\end{array}$ & Unclear risk & Not reported \\
\hline $\begin{array}{l}\text { Incomplete outcome data } \\
\text { (attrition bias) } \\
\text { All outcomes }\end{array}$ & Low risk & $\begin{array}{l}\text { In the LA group, converted cases }(6 / 61,10 \%) \text { were analysed according to ITT. } \\
\text { In both groups, a similarly low proportion of participants sent back the postal } \\
\text { questionnaire }(25 \% \text { vs. } 24 \%) \text {, but this does not affect the main outcomes of the } \\
\text { study. }\end{array}$ \\
\hline $\begin{array}{l}\text { Selective reporting (re- } \\
\text { porting bias) }\end{array}$ & Low risk & $\begin{array}{l}\text { One primary outcome (overall complication rate) was described. Other analy- } \\
\text { ses were designated as being exploratory. }\end{array}$ \\
\hline Other bias & High risk & $\begin{array}{l}\text { All laparoscopic procedures were done by surgical registrars ("Oberarzt"), } \\
\text { while open procedures were performed by registrars or experienced assistant } \\
\text { surgeons. }\end{array}$ \\
\hline
\end{tabular}

\section{Kald 1999}

\begin{tabular}{ll} 
Methods & Randomised controlled trial \\
& Centres: one university and four county hospitals (Sweden) \\
\hline Participants & $\begin{array}{l}\text { Participants: "with suspected appendicitis" } \\
\text { Gender: } 50 \% \text { females } \\
\text { Age: } 24 \text { and } 32 \text { years (median) in LA and OA } \\
\text { Histology: } 81 \% \text { inflamed }\end{array}$ \\
\hline Interventions & LA: three trocar technique; stump closure by different techniques ( $\mathrm{n}=49)$ \\
& Antibiotics: cefuroxime 1.5 g and metronidazole 500 mg IV \\
\hline Outcomes & Hospital stay; return to full activities and work; complication rates; duration of operation; costs (direct \\
& and indirect) \\
\hline Notes & $\begin{array}{l}\text { Follow-up: after } 28 \text { days. } \\
\text { No appendectomy: not reported }\end{array}$ \\
& Declaration of interest: not reported \\
& Funding: not reported \\
\hline
\end{tabular}


Kald 1999 (Continued)

Preoperative imaging: not reported

Analgesia requirements: not reported

Sample size calculation: not reported

\section{Risk of bias}

\begin{tabular}{|c|c|c|}
\hline Bias & Authors' judgement & Support for judgement \\
\hline $\begin{array}{l}\text { Random sequence genera- } \\
\text { tion (selection bias) }\end{array}$ & Low risk & Randomisation was made "in blocks of four, computer-generated,..." \\
\hline $\begin{array}{l}\text { Allocation concealment } \\
\text { (selection bias) }\end{array}$ & Low risk & "... in sequential numbers with sealed envelopes." \\
\hline $\begin{array}{l}\text { Blinding of participants } \\
\text { and personnel (perfor- } \\
\text { mance bias) } \\
\text { All outcomes }\end{array}$ & Unclear risk & Not reported \\
\hline $\begin{array}{l}\text { Blinding of outcome as- } \\
\text { sessment (detection bias) } \\
\text { All outcomes }\end{array}$ & Unclear risk & Not reported \\
\hline $\begin{array}{l}\text { Incomplete outcome data } \\
\text { (attrition bias) } \\
\text { All outcomes }\end{array}$ & High risk & $\begin{array}{l}\text { Two participants did not complete follow-up and were excluded from all } \\
\text { analyses (including short-term outcomes). One further participant with hemi- } \\
\text { colectomy after appendectomy was excluded from all analysis. Only the single } \\
\text { converted case was analysed according to ITT. }\end{array}$ \\
\hline $\begin{array}{l}\text { Selective reporting (re- } \\
\text { porting bias) }\end{array}$ & Low risk & One primary outcome measure (total costs) was described. \\
\hline Other bias & Low risk & $\begin{array}{l}\text { For trial surgeons, experience of more than } 5 \text { LAs and } 30 \text { laparoscopic chole- } \\
\text { cystectomies was demanded. }\end{array}$ \\
\hline
\end{tabular}

\section{Kaplan 2009}

\begin{tabular}{ll}
\hline Methods & Randomised controlled trial \\
& Centre: one (Turkey) \\
\hline Participants & $\begin{array}{l}\text { Patients with suspected appendicitis were included in the study. } \\
\text { Gender: } 36 \% \text { females } \\
\text { Age: } 24.2 \text { and } 26.2 \text { years (mean) in LA and OA } \\
\text { Histology: } 96 \% \text { inflamed }\end{array}$ \\
\hline Interventions & $\begin{array}{l}\text { LA: three trocar technique (10, } 5 \text { and } 5 \mathrm{~mm} \text { ) or (12, } 5 \text { and } 5 \mathrm{~mm} \text { ), "the base of the appendix is triple-lig- } \\
\text { OA: not reported ( } \mathrm{n}=43 \text { ) } \\
\text { Antibiotics: not reported }\end{array}$ \\
\hline Outcomes & $\begin{array}{l}\text { Hospital stay; need for analgesics; pain (VAS); gastrointestinal quality of life index (GIQLI); hospital } \\
\text { costs; operative time; complications }\end{array}$ \\
\hline Notes & $\begin{array}{l}\text { Follow-up: } 6 \text { weeks and } 6 \text { months after appendectomy } \\
\text { No appendectomy: All participants were resected }\end{array}$
\end{tabular}


Kaplan 2009 (Continued)

Declaration of interest: not reported

Funding: not reported

Preoperative imaging: "positive radiological findings in US or CT..."

Analgesia requirements: not reported

Sample size calculation: not reported

\section{Risk of bias}

\begin{tabular}{|c|c|c|}
\hline Bias & Authors' judgement & Support for judgement \\
\hline $\begin{array}{l}\text { Random sequence genera- } \\
\text { tion (selection bias) }\end{array}$ & Unclear risk & "[Patients] were randomly assigned either to OA or LA". \\
\hline $\begin{array}{l}\text { Allocation concealment } \\
\text { (selection bias) }\end{array}$ & Unclear risk & Not reported \\
\hline $\begin{array}{l}\text { Blinding of participants } \\
\text { and personnel (perfor- } \\
\text { mance bias) } \\
\text { All outcomes }\end{array}$ & Unclear risk & Not reported \\
\hline $\begin{array}{l}\text { Blinding of outcome as- } \\
\text { sessment (detection bias) } \\
\text { All outcomes }\end{array}$ & Unclear risk & Not reported \\
\hline $\begin{array}{l}\text { Incomplete outcome data } \\
\text { (attrition bias) } \\
\text { All outcomes }\end{array}$ & Low risk & $\begin{array}{l}\text { First follow-up after } 6 \text { weeks was completed for all } 83 \text { participants }(100 \%) \text {, but } \\
\text { dropout rates were similar between the groups (LA } n=10 ; \text { OA } n=7) \text {. Second } \\
\text { follow-up after } 6 \text { months was completed for } 66 \text { participants }(80 \%) \text {. There were } \\
\text { no conversions. }\end{array}$ \\
\hline $\begin{array}{l}\text { Selective reporting (re- } \\
\text { porting bias) }\end{array}$ & Unclear risk & No distinction between primary and secondary outcome \\
\hline Other bias & Low risk & $\begin{array}{l}\text { "All laparoscopies were undertaken or assisted by one experienced surgeon } \\
\text { who had completed at least } 100 \text { LA." }\end{array}$ \\
\hline
\end{tabular}

Karadayi 2003

\begin{tabular}{ll}
\hline Methods & Randomised controlled trial \\
& Centres: one (Turkey) \\
\hline Participants & $\begin{array}{l}\text { Participants: "with acute appendicitis" } \\
\text { Gender: } 43 \% \text { females } \\
\text { Age: } 28.9 \text { and } 28.7 \text { (mean) in LA and OA } \\
\text { Histology: unclear, since participants with normal histology or perforation were excluded }\end{array}$ \\
\hline Interventions & LA: three trocar technique; stump closure by endoloops ( $\mathrm{n}=30)$ \\
& Antibiotics: 1 g ceftriaxone and 500 mg metronidazole IV \\
\hline Outcomes & Hospital stay; complication rates; duration of operation; inflammatory markers \\
\hline Notes & Follow-up: not reported \\
\hline
\end{tabular}

Laparoscopic versus open surgery for suspected appendicitis (Review) 
No appendectomy: all appendices were obviously resected

Declaration of interest: not reported

Funding: not reported

Preoperative imaging: not reported

Analgesia requirements: not reported

Sample size calculation: not reported

\section{Risk of bias}

\begin{tabular}{lll}
\hline Bias & Authors' judgement & Support for judgement \\
\hline $\begin{array}{l}\text { Random sequence genera- } \\
\text { tion (selection bias) }\end{array}$ & Low risk & $\begin{array}{l}\text { "Patients were assigned to undergo either laparoscopic or open appendecto- } \\
\text { my on the basis of computerized, random number generation carried out out- } \\
\text { side of our department." }\end{array}$ \\
\hline $\begin{array}{ll}\text { Allocation concealment } \\
\text { (selection bias) }\end{array}$ & Unclear risk & Not reported \\
\hline $\begin{array}{l}\text { Blinding of participants } \\
\begin{array}{l}\text { and personnel (perfor- } \\
\text { mance bias) }\end{array}\end{array}$ & Unclear risk \\
\begin{tabular}{l} 
All outcomes \\
\hline
\end{tabular}
\end{tabular}

Blinding of outcome as- Unclear risk Not reported

sessment (detection bias)

All outcomes

\section{Incomplete outcome data High risk} (attrition bias)

All outcomes
In the LA and OA group, 9 and 12 participants were excluded from all analyses, due to normal histology, perforation, or conversion. In a letter dated January 2004, the author explained that no data were available for these participants, thus excluding 21 participants ( $26 \%$ of 81 ) from ITT analysis. Conversion rate was $3 / 39(8 \%)$.

\begin{tabular}{lll}
\hline $\begin{array}{l}\text { Selective reporting (re- } \\
\text { porting bias) }\end{array}$ & Low risk & Primary outcome (inflammatory markers) was adequately described. \\
\hline Other bias & Low risk & Surgeons were "experienced in laparoscopic and open surgical techniques". \\
\hline
\end{tabular}

\section{Kargar 2011}

\begin{tabular}{ll}
\hline Methods & Randomised controlled trial \\
& Centres: one (Iran) \\
\hline Participants & Participants: with a "diagnosis of acute appendicitis according to Alvorado score" \\
& Gender: $49 \%$ females \\
& Age: 27 and 25.4 years (mean) in LA and OA group \\
& Histology: $72 \%$ inflamed \\
\hline Interventions & LA: three trocar technique (10 mm umbilical, 10 mm right iliac fossa, 5 mm suprapubic); stump closure \\
& not described ( $=50)$ \\
& OA: through a McBurney incision $(n=50)$
\end{tabular}


Kargar 2011 (Continued)

Antibiotics: ceftriaxone (1 g every $12 \mathrm{~h}$ ) and metronidazole (500 mg every $8 \mathrm{~h}$ ) for $48 \mathrm{~h}$

Outcomes

Hospital stay; postoperative pain (VAS); operative time; complications; return to full activities

Notes

Follow-up: 4 weeks

No appendectomy: not reported

Declaration of interest: not reported

Funding: not reported

Preoperative imaging: not reported

Analgesia requirements: "The routine analgesic used for patients was morphine $(5 \mathrm{mg}$ intramuscular, every 8 hours)."

Sample size calculation: not reported

\section{Risk of bias}

\begin{tabular}{|c|c|c|}
\hline Bias & Authors' judgement & Support for judgement \\
\hline \multirow[t]{2}{*}{$\begin{array}{l}\text { Random sequence genera- } \\
\text { tion (selection bias) }\end{array}$} & Low risk & $\begin{array}{l}\text { "The randomization technique was by having [the] patient open a concealed } \\
\text { envelope from a randomized order of envelop[e]s [prepared] by a blinded } \\
\text { technician." }\end{array}$ \\
\hline & & $\begin{array}{l}\text { We assumed that the randomised order was adequately prepared, e.g. by shuf- } \\
\text { fling the envelopes. }\end{array}$ \\
\hline $\begin{array}{l}\text { Allocation concealment } \\
\text { (selection bias) }\end{array}$ & Low risk & $\begin{array}{l}\text { We assumed that participants were unable to foresee the next random assign- } \\
\text { ment, because envelopes were described as being "concealed". }\end{array}$ \\
\hline $\begin{array}{l}\text { Blinding of participants } \\
\text { and personnel (perfor- } \\
\text { mance bias) } \\
\text { All outcomes }\end{array}$ & High risk & The study was unblinded. \\
\hline
\end{tabular}

\begin{tabular}{lll}
$\begin{array}{l}\text { Blinding of outcome as- } \\
\text { sessment (detection bias) } \\
\text { All outcomes }\end{array}$ & High risk & The study was unblinded. \\
\hline $\begin{array}{l}\text { Incomplete outcome data } \\
\begin{array}{l}\text { (attrition bias) } \\
\text { All outcomes }\end{array}\end{array}$ & Low risk & $\begin{array}{l}\text { No exclusions or losses to follow-up reported. There were no conversions ( }= \\
0.0 \%) .\end{array}$ \\
\hline $\begin{array}{l}\text { Selective reporting (re- } \\
\text { porting bias) }\end{array}$ & Unclear risk & No distinction between primary and secondary outcome \\
\hline $\begin{array}{l}\text { Other bias } \\
\text { Unclear risk }\end{array}$ & $\begin{array}{l}\text { "All of the surgeries were performed by the same surgeon". } \\
\text { However, no information was given on how experienced this surgeon was in LA } \\
\text { and OA. }\end{array}$ \\
\hline
\end{tabular}

Katkhouda 2005

Methods Randomised controlled trial


Katkhouda 2005 (Continued)

Centres: two (USA)

Participants:older than 16 years with a diagnosis of appendicitis
Gender: $26 \%$ females
Age: 29 and 28 years (median) for LA and OA
Histology: $91 \%$ inflamed

LA: three trocar technique, dissection of mesoappendix and stump using stapling device $(\mathrm{n}=113)$
OA: McBurney muscle-splitting incision 1.5 inches in the right lower quadrant $(\mathrm{n}=134)$
Antibiotics: $1 \mathrm{~g}$ cefotaxime

Outcomes Postoperative complications; evaluation of pain and activity scores; resumption of diet; length of stay

Follow-up: "short-term"
No appendectomy: 6 normal specimens (1 in the OA and 5 in the LA group)
Declaration of interest: not reported
Funding: not reported
Preoperative imaging: not reported
Analgesia requirements: "a standardized postoperative regimen was given to all including Tylenol/
codeine capsules as a first line of treatment and shots of IM Demerol as needed." "Narcotic medication
usage to control postoperative pain was equivalent between the 2 groups."
Sample size calculation: adequately reported

\section{Risk of bias}

\begin{tabular}{|c|c|c|}
\hline Bias & Authors' judgement & Support for judgement \\
\hline $\begin{array}{l}\text { Random sequence genera- } \\
\text { tion (selection bias) }\end{array}$ & Low risk & $\begin{array}{l}\text { "Computer-generated random numbers were used to assign the type of } \\
\text { surgery (laparoscopic or open),..." }\end{array}$ \\
\hline $\begin{array}{l}\text { Allocation concealment } \\
\text { (selection bias) }\end{array}$ & Low risk & "..., which were written on a card sealed in a completely opaque envelope." \\
\hline $\begin{array}{l}\text { Blinding of participants } \\
\text { and personnel (perfor- } \\
\text { mance bias) } \\
\text { All outcomes }\end{array}$ & Low risk & $\begin{array}{l}\text { " } 3 \text { wound dressings and an abdominal binder were applied to every patient to } \\
\text { blind the patient, the nursing and the medical staff, and the independent data } \\
\text { collector as to the nature of the procedure." }\end{array}$ \\
\hline $\begin{array}{l}\text { Blinding of outcome as- } \\
\text { sessment (detection bias) } \\
\text { All outcomes }\end{array}$ & Low risk & $\begin{array}{l}\text { " } 3 \text { wound dressings and an abdominal binder were applied to every patient to } \\
\text { blind the patient, the nursing and the medical staff, and the independent data } \\
\text { collector as to the nature of the procedure." }\end{array}$ \\
\hline $\begin{array}{l}\text { Incomplete outcome data } \\
\text { (attrition bias) } \\
\text { All outcomes }\end{array}$ & High risk & $\begin{array}{l}\text { Completeness of follow-up was not described. All } 134 \text { participants in the OA } \\
\text { group were included in the analyses. In the LA group, converted cases ( } 9 / 113 \text {, } \\
8 \% \text { ) were analysed according to ITT. However, } 11 \text { dropped out of the LA group } \\
\text { after being randomised: } 10 \text { participants refused the assigned operation and } 1 \\
\text { woman was pregnant. Another } 10 \text { participants, again all in the LA group, were } \\
\text { excluded because of missing data. In summary, therefore, } 21 \text { of } 134 \text { partici- } \\
\text { pants in the LA group (16\%) were removed from the analyses. }\end{array}$ \\
\hline
\end{tabular}

$\begin{array}{ll}\begin{array}{l}\text { Selective reporting (re- } \\ \text { porting bias) }\end{array} & \text { Low risk } \\ \end{array}$


Katkhouda 2005 (Continued)
Other bias
Low risk
"Residents performed all operations with 4 attending surgeons experienced in open and advanced laparoscopic techniques".

\section{Kazemier 1997}

\begin{tabular}{ll}
\hline Methods & Randomised controlled trial \\
& Centres: two (Netherlands) \\
\hline Participants & Participants: with "a clinical diagnosis of acute appendicitis" \\
& Gender: $45 \%$ females \\
& Age: 30.8 and 33.7 years (mean) in LA and OA \\
& Histology: $87 \%$ inflamed
\end{tabular}

Interventions

LA: three trocar technique; stump closure by endo loops (or stapling device in heavily inflamed cases) $(\mathrm{n}=97)$

OA: via muscle splitting incision $(n=104)$

Antibiotics: $1 \mathrm{~g}$ cefotaxime and $500 \mathrm{mg}$ metronidazole IV

\begin{tabular}{ll}
\hline Outcomes & $\begin{array}{l}\text { Hospital stay; return to full activities and work; return to normal diet; complication rates; duration of } \\
\text { operation; pain scores on day } 1 \text { and } 2 \text { (VAS); consumption of analgesics }\end{array}$ \\
\hline Notes & Follow-up: not reported \\
No appendectomy: in one participant \\
Declaration of interest: not reported \\
Funding: not reported \\
Preoperative imaging: not reported \\
Analgesia requirements: "... 1 mg/kg pethidine, maximally every 6 h on the 1 st day and $1 \mathrm{~g}$ of paraceta- \\
mol, maximally every $6 \mathrm{~h}$ on the $2 \mathrm{nd}$ postoperative day." "Postoperative pain was significantly less in \\
the LA group on both the 1 st and 2 nd postoperative day, resulting in lower scores on the VAS and in less \\
use of analgesics." \\
Sample size calculation: not reported
\end{tabular}

\section{Risk of bias}

\begin{tabular}{|c|c|c|}
\hline Bias & Authors' judgement & Support for judgement \\
\hline $\begin{array}{l}\text { Random sequence genera- } \\
\text { tion (selection bias) }\end{array}$ & Low risk & "Computer-generated blocked random numbers..." \\
\hline $\begin{array}{l}\text { Allocation concealment } \\
\text { (selection bias) }\end{array}$ & Low risk & "by drawing a card from an opaque envelope" \\
\hline $\begin{array}{l}\text { Blinding of participants } \\
\text { and personnel (perfor- } \\
\text { mance bias) } \\
\text { All outcomes }\end{array}$ & Unclear risk & Not reported \\
\hline $\begin{array}{l}\text { Blinding of outcome as- } \\
\text { sessment (detection bias) } \\
\text { All outcomes }\end{array}$ & Unclear risk & Not reported \\
\hline
\end{tabular}


Kazemier 1997 (Continued)

Incomplete outcome data Low risk No exclusions or losses to follow-up reported. Converted cases $(n=12,12 \%)$ (attrition bias) and those receiving other surgery were analysed by ITT.

All outcomes

\begin{tabular}{lll}
$\begin{array}{l}\text { Selective reporting (re- } \\
\text { porting bias) }\end{array}$ & Unclear risk & No distinction between primary and secondary outcome \\
\hline Other bias & Low risk & $\begin{array}{l}\text { Surgeons had "experience of more than } 15 \text { open and laparoscopic appendec- } \\
\text { tomies." }\end{array}$ \\
\hline
\end{tabular}

\section{Kehagias 2009}

$\begin{array}{ll}\text { Methods } & \text { Randomised controlled trial } \\ \text { Centres: not reported (Greece) }\end{array}$

\begin{tabular}{ll}
\hline Participants & $\begin{array}{l}\text { Participants: "Patients with clinical diagnosis of appendicitis and radiologic imaging of complicated } \\
\text { disease" } \\
\text { Gender: not reported } \\
\text { Age: not reported } \\
\text { Histology: not reported }\end{array}$ \\
\hline LA: not reported \\
OA: not reported \\
Antibiotics: not reported \\
\hline Operating time; severity of disease; hospital stay; complication rate \\
\hline Notes & Follow-up: not reported \\
No appendectomy: not reported \\
Declaration of interest: not reported \\
Funding: not reported \\
Preoperative imaging: not reported \\
Analgesia requirements: not reported \\
Sample size calculation: not reported
\end{tabular}

\section{Risk of bias}

\begin{tabular}{lll}
\hline Bias & Authors' judgement & Support for judgement \\
\hline $\begin{array}{l}\text { Random sequence genera- } \\
\text { tion (selection bias) }\end{array}$ & Low risk & By random picking of sealed envelopes (see following field) \\
\hline $\begin{array}{l}\text { Allocation concealment } \\
\text { (selection bias) }\end{array}$ & Low risk & $\begin{array}{l}\text { In written communication (September 19th, 2009), one of the trial authors } \\
\text { (S.N. Karamanakos) stated that after informed consent "the patient is asked to } \\
\text { draw from a pool of sealed envelops where in a card the type of the procedure } \\
\text { is written." }\end{array}$
\end{tabular}

$\begin{array}{ll}\begin{array}{l}\text { Blinding of participants } \\ \text { and personnel (perfor- }\end{array} & \text { Unclear risk }\end{array} \quad \begin{aligned} & \text { "Binding included both the surgical team and the patient." } \\ & \text { Comment: A detailed description of blinding was missing. }\end{aligned}$

mance bias)

All outcomes 
Khalil 2011 (Continued)

\begin{tabular}{|c|c|c|}
\hline $\begin{array}{l}\text { Blinding of participants } \\
\text { and personnel (perfor- } \\
\text { mance bias) } \\
\text { All outcomes }\end{array}$ & High risk & The study was not blinded. \\
\hline $\begin{array}{l}\text { Blinding of outcome as- } \\
\text { sessment (detection bias) } \\
\text { All outcomes }\end{array}$ & High risk & The study was not blinded. \\
\hline $\begin{array}{l}\text { Incomplete outcome data } \\
\text { (attrition bias) } \\
\text { All outcomes }\end{array}$ & High risk & $\begin{array}{l}\text { Losses to follow-up were reported for laparoscopic }(8 \text { of } 80,10 \%) \text { and open } \\
\text { group ( } 5 \text { of } 80,6 \%) \text {. These } 13 \text { participants were excluded from all analyses. } \\
\text { Participants with negative appendectomy }(n=6,4 \%) \text { and converted cases ( } n= \\
1,1 \%) \text { were analysed by ITT. }\end{array}$ \\
\hline $\begin{array}{l}\text { Selective reporting (re- } \\
\text { porting bias) }\end{array}$ & Unclear risk & $\begin{array}{l}\text { The study had } 3 \text { primary outcome measures, without any adjustment for mul- } \\
\text { tiple hypothesis testing. }\end{array}$ \\
\hline Other bias & Low risk & $\begin{array}{l}\text { "The patients were operated by a single consultant surgeon }[. . .] \text { with sufficient } \\
\text { capability of performing the two procedures (LA and OA)." }\end{array}$ \\
\hline
\end{tabular}

\section{Kocatas 2013}

\begin{tabular}{|c|c|}
\hline Methods & $\begin{array}{l}\text { Randomised controlled trial } \\
\text { Centres: one (Turkey) }\end{array}$ \\
\hline Participants & $\begin{array}{l}\text { Participants: "diagnosed with acute uncomplicated appendicitis" } \\
\text { Gender: } 28 \% \text { females } \\
\text { Age: } 27.4 \text { and } 28.2 \text { years (mean) in LA and OA } \\
\text { Histology: not reported }\end{array}$ \\
\hline Interventions & $\begin{array}{l}\text { LA: three tocar technique }(10,5 \text {, and } 5 \mathrm{~mm}) \text {, stump closure technique not described }(n=50) \\
\text { OA: via McBurney or Rockey-Davis incision }(n=46) \\
\text { Antibiotics: single shot } 1 \text { g cefazolin }\end{array}$ \\
\hline Outcomes & $\begin{array}{l}\text { Primary outcomes: readmissions, rehospitalization, reoperations } \\
\text { Secondary outcomes: length of hospital stay, pain scores (postoperative 4th hour and at discharge, } \\
\text { VAS), QoL (Nottingham Health Profile) }\end{array}$ \\
\hline Notes & $\begin{array}{l}\text { Follow-up: } 30 \text { days after surgery } \\
\text { No appendectomy: none described } \\
\text { Declaration of interest: the authors declared no conflicts of interest } \\
\text { Funding: not reported } \\
\text { Preoperative imaging: "All of the patients underwent combined clinical, radiological, and biochemical } \\
\text { evaluations for suspected appendicitis." } \\
\text { Analgesia requirements: "Tramadol ( } 100 \text { mg as needed, intravenous) was administered for the man- } \\
\text { agement of postoperative pain and was replaced by peroral naproxen sodium ( } 550 \text { mg twice a day) af- } \\
\text { ter the initiation of oral intake." Consumption of analgesia was not reported. }\end{array}$ \\
\hline
\end{tabular}




\section{Risk of bias}

Bias Authors' judgement Support for judgement

Random sequence genera- Low risk tion (selection bias)

"Randomisation was performed using a lottery method. A resident, who had no knowledge of the preoperative data and would not be involved in the operations, was chosen to select lottery cards."

In an email dated June 4th, 2013, the author stated: "One of the medical staff in the operating theater who had no idea about the patient was asked to put two pieces of paper, written $L$ on one and $O$ on the other, within an opaque bag before the operation. And the card picker was the anesthesiologist. Old papers were never put back into the bag, new papers were prepared for each patient."

Allocation concealment $\quad$ Low risk See previous entry
(selection bias)

Blinding of participants $\quad$ Unclear risk Not reported
and personnel (perfor-
mance bias)
All outcomes

Blinding of outcome as- Unclear risk Not reported sessment (detection bias)

All outcomes

\begin{tabular}{|c|c|c|}
\hline $\begin{array}{l}\text { Incomplete outcome data } \\
\text { (attrition bias) } \\
\text { All outcomes }\end{array}$ & Unclear risk & $\begin{array}{l}\text { In an email dated June } 4 \text { th, } 2013 \text {, the author stated: "We did not encounter } \\
\text { any conversion to open technique throughout the study". However, three par- } \\
\text { ticipants were lost to follow-up and excluded from the analysis. }\end{array}$ \\
\hline
\end{tabular}

\begin{tabular}{lll}
\hline $\begin{array}{l}\text { Selective reporting (re- } \\
\text { porting bias) }\end{array}$ & Low risk & $\begin{array}{l}\text { The primary outcome (complication rate) was adequately described. The } \\
\text { study was therefore judged to have low risk of bias, although neither a study } \\
\text { protocol nor a study registration were available. }\end{array}$ \\
\hline Other bias & Low risk & $\begin{array}{l}\text { In an email dated June 4th, 2013, the author stated: "The surgeons are quite } \\
\text { experienced in appendectomy procedure. Approximately } 50 \text { appendectomies } \\
\text { are performed each month. A vast majority of these are done laparoscopical- } \\
\text { ly." }\end{array}$
\end{tabular}

Kouhia 2010

\begin{tabular}{ll}
\hline Methods & Randomised controlled trial \\
& Centres: one (Finland) \\
\hline Participants & Participants: Patients over 15 years old with suspected acute appendicitis were included. Men with nor- \\
& mal weight (body mass index $<$ or $=25 \mathrm{~kg} / \mathrm{m}^{2}$ ) were not included. \\
& Gender: $84 \%$ females \\
& Age: 28 and 34 years (median) in LA and OA \\
& Histology: $71 \%$ inflamed \\
\hline
\end{tabular}

Interventions

LAP: A normal appendix was left in situ, if another cause for abdominal pain was found.

LA: three trocar technique $(10,5$, and $10 \mathrm{~mm})$; stump closure by three loops $(\mathrm{n}=47)$ 
Kouhia 2010 (Continued)

OA: "via a right iliac fossa incision" $(n=52)$

Antibiotics: metronidazole $500 \mathrm{mg}$ IV

\begin{tabular}{ll}
\hline Outcomes & $\begin{array}{l}\text { Hospital stay; return to work; return to normal diet; complication rates (including readmissions and } \\
\text { long-term results); duration of operation; blood loss }\end{array}$ \\
\hline Notes & Follow-up: 4 weeks \\
No appendectomy: was needed in 6 of $105(6 \%)$ participants, who were excluded from analysis \\
Declaration of interest: "The authors declare no conflict of interest." \\
Funding: North Karelia Central Hospital \\
Preoperative imaging: not reported \\
Analgesia requirements: no difference between the two groups (details not reported)Sample size calcu- \\
lation: not reported
\end{tabular}

\section{Risk of bias}

\begin{tabular}{lll}
\hline Bias & Authors' judgement & Support for judgement \\
\hline $\begin{array}{l}\text { Random sequence genera- } \\
\text { tion (selection bias) }\end{array}$ & Unclear risk & "Patients were randomized to OA or LA ..." \\
\hline $\begin{array}{l}\text { Allocation concealment } \\
\text { (selection bias) }\end{array}$ & Low risk & $\begin{array}{l}\text { "Treatment allocations were sealed in numbered envelopes that were opened } \\
\text { in sequence." }\end{array}$ \\
\hline $\begin{array}{l}\text { Blinding of participants } \\
\text { and personnel (perfor- } \\
\text { mance bias) } \\
\begin{array}{l}\text { All outcomes } \\
\hline\end{array}\end{array}$ & Unclear risk & Not reported \\
\hline
\end{tabular}

Blinding of outcome as- Unclear risk Not reported

sessment (detection bias)

All outcomes

Incomplete outcome data High risk (attrition bias)

All outcomes
Conversion occurred in the LA group $(n=3,6 \%)$ and in the OA group $(n=1$, converted to midline laparotomy). These participants were analysed by ITT.

After randomisation, however, 6 participants, in whom other diseases were found, were excluded from analysis ( 2 in OA group and 4 in LA group).

Selective reporting (re- Unclear risk
porting bias)

No distinction between primary and secondary outcomes. The trial was registered (NCT00908804) after being performed, but this was because trial registries did not exist when the trial was begun.
"In the present study, trainees performed OA significantly more often than consultants (and vice versa for LA). This may have influenced the duration of operation. [...] The different distribution of surgeon grade between the study groups is a methodological limitation."

$\begin{array}{ll}\text { Methods } & \text { Randomised controlled trial } \\ \text { Centres: one (Singapore) }\end{array}$


Kum 1993a (Continued)

Participants
Gender: $69 \%$ females
Age: 33.1 and 30.7 years (mean) in LA and OA
Histology: $85 \%$ inflamed

LAP: If other causes of abdominal pain were found, the appendix was not removed. LA: stump closure
by endo loops $(n=52)$
OA: "standard right lower quadrant incision" ( $n=57)$
Antibiotics: gentamicin and metronidazole (dose not stated)

Outcomes Hospital stay; return to full activities and work; return to normal diet; complication rates; duration of operation; pain scores on day 1 and 2 (VAS); consumption of analgesics;informal cost analysis (direct costs)

Notes Follow-up: after 1 week and 1 month, if necessary after 2 months

No appendectomy: In 10 LA participants, the appendix was not removed.

Declaration of interest: not reported

Funding: not reported

Preoperative imaging: not reported

Analgesia requirements: "After the operation, intramuscular pethidine $1 \mathrm{mg} / \mathrm{kg}$ every $4 \mathrm{~h}$ was given on demand. Oral naproxen sodium was given as a $550 \mathrm{mg}$ dose twice a day on demand..." "...no difference in the number of doses of pethidine requested by the two groups."

Sample size calculation: not reported

\section{Risk of bias}

\begin{tabular}{|c|c|c|}
\hline Bias & Authors' judgement & Support for judgement \\
\hline $\begin{array}{l}\text { Random sequence genera- } \\
\text { tion (selection bias) }\end{array}$ & Low risk & $\begin{array}{l}\text { "Randomization was achieved with the toss of a coin by independent ob- } \\
\text { servers." }\end{array}$ \\
\hline $\begin{array}{l}\text { Allocation concealment } \\
\text { (selection bias) }\end{array}$ & Low risk & $\begin{array}{l}\text { "Randomization was achieved with the toss of a coin by independent ob- } \\
\text { servers." }\end{array}$ \\
\hline $\begin{array}{l}\text { Blinding of participants } \\
\text { and personnel (perfor- } \\
\text { mance bias) } \\
\text { All outcomes }\end{array}$ & Unclear risk & Not reported \\
\hline $\begin{array}{l}\text { Blinding of outcome as- } \\
\text { sessment (detection bias) } \\
\text { All outcomes }\end{array}$ & Unclear risk & Not reported \\
\hline $\begin{array}{l}\text { Incomplete outcome data } \\
\text { (attrition bias) } \\
\text { All outcomes }\end{array}$ & High risk & $\begin{array}{l}\text { Cases in whom no appendectomy was necessary }(n=10) \text { and those with a his- } \\
\text { tologically normal }(n=11) \text { or perforated appendix }(n=7) \text { were excluded from } \\
\text { analysis. Thus, } 28 \text { participants ( } 20 \% \text { of } 137) \text { were not analysed by ITT. }\end{array}$ \\
\hline $\begin{array}{l}\text { Selective reporting (re- } \\
\text { porting bias) }\end{array}$ & Unclear risk & No distinction between primary and secondary outcome \\
\hline Other bias & Low risk & $\begin{array}{l}\text { Trial surgeons were "registrars or residents with experience of at least } 6 \\
\text { months". }\end{array}$ \\
\hline
\end{tabular}


Laine 1997

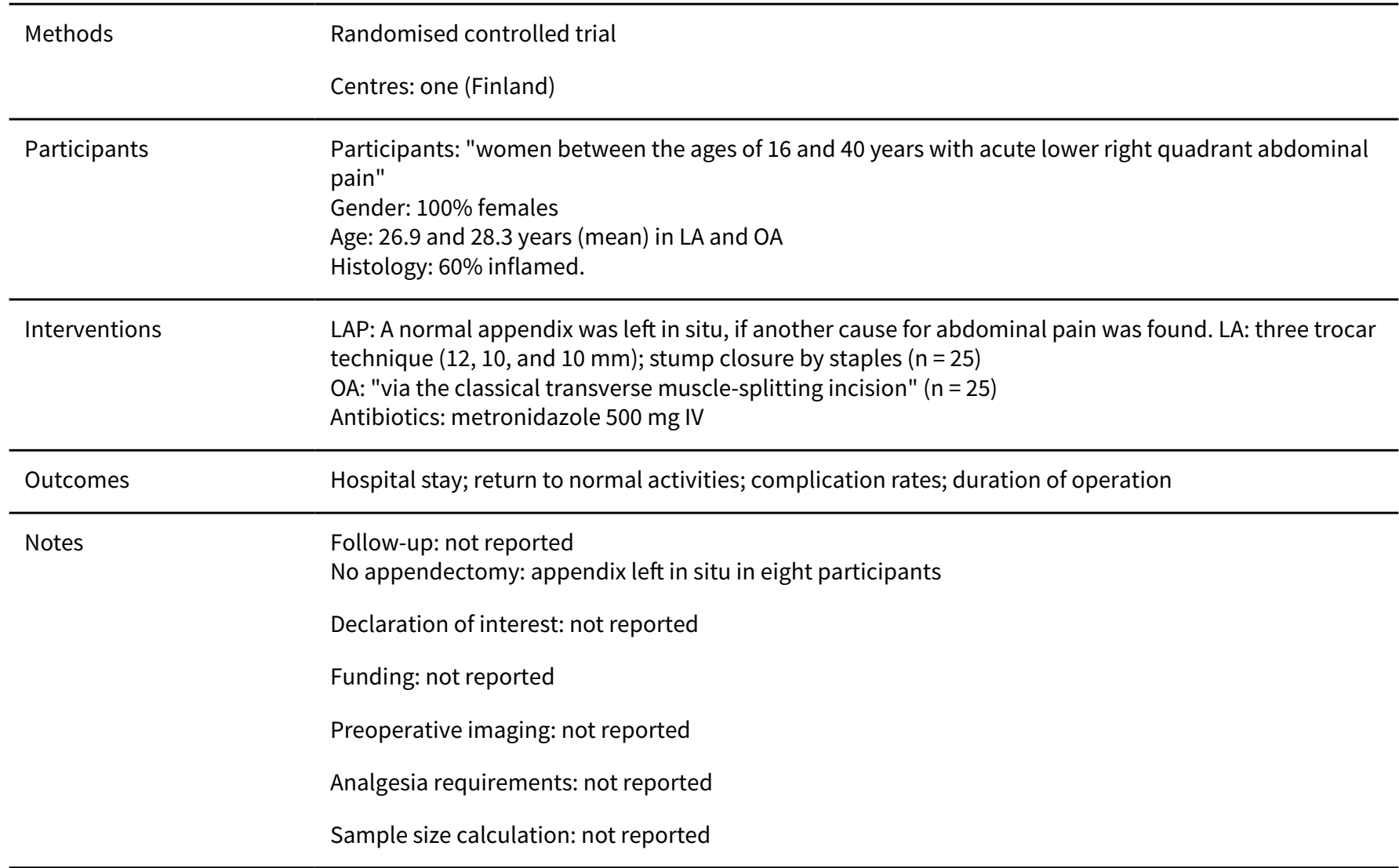

\section{Risk of bias}

\begin{tabular}{|c|c|c|}
\hline Bias & Authors' judgement & Support for judgement \\
\hline $\begin{array}{l}\text { Random sequence genera- } \\
\text { tion (selection bias) }\end{array}$ & Unclear risk & "The goal of this randomized study..." \\
\hline $\begin{array}{l}\text { Allocation concealment } \\
\text { (selection bias) }\end{array}$ & Unclear risk & Not reported \\
\hline $\begin{array}{l}\text { Blinding of participants } \\
\text { and personnel (perfor- } \\
\text { mance bias) } \\
\text { All outcomes }\end{array}$ & Unclear risk & Not reported \\
\hline $\begin{array}{l}\text { Blinding of outcome as- } \\
\text { sessment (detection bias) } \\
\text { All outcomes }\end{array}$ & Unclear risk & Not reported \\
\hline $\begin{array}{l}\text { Incomplete outcome data } \\
\text { (attrition bias) } \\
\text { All outcomes }\end{array}$ & Low risk & All patients including converted cases $(n=2,8 \%)$ were analysed by ITT. \\
\hline $\begin{array}{l}\text { Selective reporting (re- } \\
\text { porting bias) }\end{array}$ & Unclear risk & No distinction between primary and secondary outcome \\
\hline Other bias & High risk & The surgical resident on call performed the operation. \\
\hline
\end{tabular}


Larsson 2001

\begin{tabular}{ll} 
Methods & Randomised controlled trial \\
& Centres: one (Sweden) \\
\hline Participants & Participants: women aged 15 to 47 with clinical signs of acute appendicitis \\
& Gender: females only 24.9 and 25 years (mean) in LAP and OA \\
\hline Interventions & LAP: Diagnostic laparoscopy followed by open appendectomy when acute appendicitis was visible ( $\mathrm{n}=$ \\
& OA) via right lower quadrant incision ( $\mathrm{n}=55)$ \\
& Antibiotics: $1 \mathrm{~g}$ metronidazole rectally \\
\hline Rutcomes of unnecessary appendectomies and participants without diagnosis established & Follow-up: not specified \\
Conversions: in one participant, the appendix could not be visualised & Declaration of interest: not reported \\
Fotes & Funding: not reported \\
& Preoperative imaging: not reported \\
Analgesia requirements: not reported & Sample size calculation: not reported
\end{tabular}

\section{Risk of bias}

\begin{tabular}{|c|c|c|}
\hline Bias & Authors' judgement & Support for judgement \\
\hline $\begin{array}{l}\text { Random sequence genera- } \\
\text { tion (selection bias) }\end{array}$ & Unclear risk & "Patients were randomized by the anaesthesiologic nurse..." \\
\hline $\begin{array}{l}\text { Allocation concealment } \\
\text { (selection bias) }\end{array}$ & Low risk & "... using sealed envelopes in blocks of 10 patients" \\
\hline $\begin{array}{l}\text { Blinding of participants } \\
\text { and personnel (perfor- } \\
\text { mance bias) } \\
\text { All outcomes }\end{array}$ & Unclear risk & Not reported \\
\hline $\begin{array}{l}\text { Blinding of outcome as- } \\
\text { sessment (detection bias) } \\
\text { All outcomes }\end{array}$ & Unclear risk & Not reported \\
\hline $\begin{array}{l}\text { Incomplete outcome data } \\
\text { (attrition bias) } \\
\text { All outcomes }\end{array}$ & High risk & $\begin{array}{l}\text { "Two patients were excluded, one because of incomplete follow-up evaluation } \\
\text { and another because of protocol violation, as she was scheduled for an explo- } \\
\text { rative laparotomy regardless of the laparoscopic finding." Thus, the ITT princi- } \\
\text { ple was violated. }\end{array}$ \\
\hline $\begin{array}{l}\text { Selective reporting (re- } \\
\text { porting bias) }\end{array}$ & Unclear risk & No distinction between primary and secondary outcome \\
\hline Other bias & High risk & Surgeon and gynaecologist on call performed the operation. \\
\hline
\end{tabular}


Lavonius 2001

\begin{tabular}{ll} 
Methods & Randomised controlled trial \\
& Centres: two (Finland) \\
\hline Participants & $\begin{array}{l}\text { Participants: children aged } 7 \text { to } 15 \mathrm{yrs} \text {, with suspected acute appendicitis, but not diffuse peritonitis } \\
\text { Gender: } 33 \% \text { girls } \\
\text { Age: } 12.5 \text { and } 11.9 \text { years (mean) in LA and OA } \\
\text { Histology: } 93 \% \text { inflamed }\end{array}$ \\
\hline Interventions & LA: three trocar technique, stump closure by varying techniques $(\mathrm{n}=23)$ \\
& Antibiotics: 7 mg/kg metronidazole IV in all participants \\
\hline Outcomes & Hospital stay; return to normal activities; complication rates; duration of operation; gut function \\
\hline Notes & Follow-up: by telephone after 1 and 4 weeks (86\% and $84 \%$ complete) \\
& No appendectomy: "All appendices were removed." \\
& Declaration of interest: not reported \\
& Funding: not reported \\
& Preoperative imaging: not reported \\
Analgesia requirements: not reported & Sample size calculation: not reported \\
\end{tabular}

\section{Risk of bias}

\begin{tabular}{|c|c|c|}
\hline Bias & Authors' judgement & Support for judgement \\
\hline $\begin{array}{l}\text { Random sequence genera- } \\
\text { tion (selection bias) }\end{array}$ & Unclear risk & "The patients were randomised..." \\
\hline $\begin{array}{l}\text { Allocation concealment } \\
\text { (selection bias) }\end{array}$ & Low risk & "...with closed envelopes" \\
\hline $\begin{array}{l}\text { Blinding of participants } \\
\text { and personnel (perfor- } \\
\text { mance bias) } \\
\text { All outcomes }\end{array}$ & Unclear risk & Not reported \\
\hline $\begin{array}{l}\text { Blinding of outcome as- } \\
\text { sessment (detection bias) } \\
\text { All outcomes }\end{array}$ & Unclear risk & Not reported \\
\hline $\begin{array}{l}\text { Incomplete outcome data } \\
\text { (attrition bias) } \\
\text { All outcomes }\end{array}$ & Low risk & $\begin{array}{l}\text { Out of a total of } 43 \text { children, } 7 \text { were lost to follow-up after } 4 \text { weeks, but main } \\
\text { results were based on all randomised participants. The single converted case } \\
\text { was analysed by ITT. }\end{array}$ \\
\hline $\begin{array}{l}\text { Selective reporting (re- } \\
\text { porting bias) }\end{array}$ & Unclear risk & No distinction between primary and secondary outcome \\
\hline Other bias & Low risk & For trial surgeons, a minimum experience of $10 \mathrm{LAs}$ in adults was required. \\
\hline
\end{tabular}


Lejus 1996

\begin{tabular}{|c|c|}
\hline Methods & $\begin{array}{l}\text { Randomised controlled trial } \\
\text { Centres: one (France) }\end{array}$ \\
\hline Participants & $\begin{array}{l}\text { Participants: "children, aged } 8-15 \text { yrs, with clinical signs of appendicitis". } \\
\text { Gender: } 46 \% \text { girls } \\
\text { Age: } 10.9 \text { and } 11.3 \text { years (mean) in LA and OA } \\
\text { Histology: } 75 \% \text { inflamed }\end{array}$ \\
\hline Interventions & $\begin{array}{l}\text { LA: three trocar technique, stump closure by endoloops }(n=32) \\
\text { OA: McBurney's incision }(n=31) \\
\text { Antibiotics: not reported }\end{array}$ \\
\hline Outcomes & $\begin{array}{l}\text { Analgesic requirements on PCA; pain (VAS); shoulder pain; duration of operation and anaesthesia; com- } \\
\text { plication rates; return to walking ability }\end{array}$ \\
\hline \multirow[t]{6}{*}{ Notes } & $\begin{array}{l}\text { Follow-up: study period was } 3 \text { days, further follow-up not explained } \\
\text { No appendectomy: none reported }\end{array}$ \\
\hline & Declaration of interest: not reported \\
\hline & Funding: not reported \\
\hline & Preoperative imaging: not reported \\
\hline & $\begin{array}{l}\text { Analgesia requirements: in the recovery room: propacetamol } 25 \mathrm{mg} / \mathrm{kg} \text {, afterwards propacetamol } 25 \\
\mathrm{mg} / \mathrm{kg} \text { every } 6 \text { hours for } 48 \mathrm{~h} \text {, oral paracetamol } 12.5 \mathrm{mg} / \mathrm{kg} \text { for the next } 24 \mathrm{~h} \text {, rescue analgesia: nal- } \\
\text { buphine } 0.2 \mathrm{mg} / \mathrm{kg} \text { for } 4 \mathrm{~h} \text {. No difference between the two groups on day } 1 \text { and day } 2 \text {. }\end{array}$ \\
\hline & Sample size calculation: not reported \\
\hline
\end{tabular}

\section{Risk of bias}

\begin{tabular}{|c|c|c|}
\hline Bias & Authors' judgement & Support for judgement \\
\hline $\begin{array}{l}\text { Random sequence genera- } \\
\text { tion (selection bias) }\end{array}$ & Unclear risk & "In the operating room, children were randomly assigned..." \\
\hline $\begin{array}{l}\text { Allocation concealment } \\
\text { (selection bias) }\end{array}$ & Unclear risk & Not reported \\
\hline $\begin{array}{l}\text { Blinding of participants } \\
\text { and personnel (perfor- } \\
\text { mance bias) } \\
\text { All outcomes }\end{array}$ & Low risk & $\begin{array}{l}\text { "Children, parents, and nurses did not know during the study if laparoscopic } \\
\text { or open appendectomy was performed." } \\
\text { "Surgical dressings were identical for all children." }\end{array}$ \\
\hline $\begin{array}{l}\text { Blinding of outcome as- } \\
\text { sessment (detection bias) } \\
\text { All outcomes }\end{array}$ & Unclear risk & Not reported \\
\hline $\begin{array}{l}\text { Incomplete outcome data } \\
\text { (attrition bias) } \\
\text { All outcomes }\end{array}$ & Low risk & $\begin{array}{l}\text { No losses from follow-up visible. No conversions or other changes in surgical } \\
\text { procedure. }\end{array}$ \\
\hline $\begin{array}{l}\text { Selective reporting (re- } \\
\text { porting bias) }\end{array}$ & Unclear risk & No distinction between primary and secondary outcome \\
\hline Other bias & Unclear risk & The surgeon's skills were not described \\
\hline
\end{tabular}


Lintula 2004

\begin{tabular}{|c|c|}
\hline Methods & $\begin{array}{l}\text { Randomised controlled trial } \\
\text { Centres: one (Finland) }\end{array}$ \\
\hline Participants & $\begin{array}{l}\text { Participants: children, aged } 4-15 \text { yrs, with suspected uncomplicated appendicitis } \\
\text { Gender: } 33 \text { girls ( } 38 \%) \\
\text { Age: } 11 \text { and } 12 \text { years (mean) in LA and OA } \\
\text { Histology: } 78 \% \text { inflamed }\end{array}$ \\
\hline Interventions & $\begin{array}{l}\text { LA: three trocar technique, stump closure by endoloops }(n=43) \\
\text { OA: via McBurney incision }(n=44) \\
\text { Antibiotics: metronidazole } 7 \mathrm{mg} / \mathrm{kg} \mathrm{IV}\end{array}$ \\
\hline Outcomes & $\begin{array}{l}\text { Hospital stay; complication rates; duration of operation; pain at rest and on cough ( } 0 \text { to } 10 \text { scale); anal- } \\
\text { gesic consumption; total costs }\end{array}$ \\
\hline \multirow[t]{6}{*}{ Notes } & $\begin{array}{l}\text { Follow-up: "by a follow-up call } 4 \text { weeks after operation" } \\
\text { No appendectomy: none }\end{array}$ \\
\hline & Declaration of interest: not reported \\
\hline & Funding: "This trial was not financially supported by any external source." \\
\hline & Preoperative imaging: not reported \\
\hline & Analgesia requirements: not reported \\
\hline & Sample size calculation: not reported \\
\hline
\end{tabular}

\section{Risk of bias}

\begin{tabular}{|c|c|c|}
\hline Bias & Authors' judgement & Support for judgement \\
\hline $\begin{array}{l}\text { Random sequence genera- } \\
\text { tion (selection bias) }\end{array}$ & Unclear risk & "Children were selected randomly..." \\
\hline $\begin{array}{l}\text { Allocation concealment } \\
\text { (selection bias) }\end{array}$ & Low risk & "... by sealed envelope method" \\
\hline $\begin{array}{l}\text { Blinding of participants } \\
\text { and personnel (perfor- } \\
\text { mance bias) } \\
\text { All outcomes }\end{array}$ & Unclear risk & Single-blind by using identical wound dressings \\
\hline $\begin{array}{l}\text { Blinding of outcome as- } \\
\text { sessment (detection bias) } \\
\text { All outcomes }\end{array}$ & Unclear risk & Not reported \\
\hline $\begin{array}{l}\text { Incomplete outcome data } \\
\text { (attrition bias) } \\
\text { All outcomes }\end{array}$ & High risk & $\begin{array}{l}\text { Completeness of } 4 \text { week follow-up was not reported. } 12 \text { ( } 7 \text { vs. } 5 \text { ) of } 73 \text { partici- } \\
\text { pants were excluded after randomisation due to peritonitis. This did not follow } \\
\text { the ITT principle. }\end{array}$ \\
\hline $\begin{array}{l}\text { Selective reporting (re- } \\
\text { porting bias) }\end{array}$ & Unclear risk & Primary outcome (total costs) were adequately described. \\
\hline Other bias & Low risk & $\begin{array}{l}\text { Surgeons had to have "experience with more than } 30 \text { LAs" AND "experience } \\
\text { with more than } 200 \text { OAs". }\end{array}$ \\
\hline
\end{tabular}


Little 2002

\begin{tabular}{|c|c|}
\hline Methods & $\begin{array}{l}\text { Randomised controlled trial } \\
\text { Centres: one (USA) }\end{array}$ \\
\hline Participants & $\begin{array}{l}\text { Particpants: children, aged } 1 \text { to } 16 \text { yrs, "with a preliminary diagnosis of acute appendicitis" } \\
\text { Gender: } 51 \% \text { girls } \\
\text { Age: } 12 \text { and } 10.5 \text { years (mean) in LA and OA } \\
\text { Histology: } 82 \% \text { inflamed }\end{array}$ \\
\hline Interventions & $\begin{array}{l}\text { LA: three trocar technique, trocar size varied with childrens' age, endo loops ( } \mathrm{n}=44) \\
\text { OA: through a 3- to 4-cm McBurney's muscle-splitting incision }(n=44) \\
\text { Antibiotics: "Antibiotics, when used, consisted of gentamycin, clindamycin, and ampicillin." }\end{array}$ \\
\hline Outcomes & $\begin{array}{l}\text { Hospital stay; complication rates; duration of operation; return to normal activities; analgesic con- } \\
\text { sumption; costs }\end{array}$ \\
\hline Notes & $\begin{array}{l}\text { Follow-up: "in the clinic } 2 \text { weeks postoperatively", } 80 \% \text { complete } \\
\text { No appendectomy: not reported } \\
\text { Declaration of interest: not reported } \\
\text { Funding: not reported } \\
\text { Preoperative imaging: not reported } \\
\text { Analgesia requirements: "..dose of morphine, acetaminophen or an enteral narcotic." No difference in } \\
\text { the consumption of analgesia } \\
\text { Sample size calculation: not reported }\end{array}$ \\
\hline
\end{tabular}

\section{Risk of bias}

\begin{tabular}{|c|c|c|}
\hline Bias & Authors' judgement & Support for judgement \\
\hline $\begin{array}{l}\text { Random sequence genera- } \\
\text { tion (selection bias) }\end{array}$ & Unclear risk & "Randomization was determined ..." \\
\hline $\begin{array}{l}\text { Allocation concealment } \\
\text { (selection bias) }\end{array}$ & Low risk & "... by sealed assignment card located in the operating room control desk" \\
\hline $\begin{array}{l}\text { Blinding of participants } \\
\text { and personnel (perfor- } \\
\text { mance bias) } \\
\text { All outcomes }\end{array}$ & Unclear risk & Not reported \\
\hline $\begin{array}{l}\text { Blinding of outcome as- } \\
\text { sessment (detection bias) } \\
\text { All outcomes }\end{array}$ & Unclear risk & Not reported \\
\hline $\begin{array}{l}\text { Incomplete outcome data } \\
\text { (attrition bias) } \\
\text { All outcomes }\end{array}$ & Low risk & $\begin{array}{l}\text { Two-week follow-up was complete for } 70 \text { of } 88 \text { participants but the distribu- } \\
\text { tion among the two groups was not described. Conversions }(n=3,7 \%) \text { were } \\
\text { analysed by ITT. }\end{array}$ \\
\hline $\begin{array}{l}\text { Selective reporting (re- } \\
\text { porting bias) }\end{array}$ & Unclear risk & No distinction between primary and secondary outcome \\
\hline Other bias & High risk & "Residents performed all appendectomies". \\
\hline
\end{tabular}


Long 2000

\begin{tabular}{ll}
\hline Methods & Randomised controlled trial \\
& Centres: two (?) (USA) \\
\hline Participants & Participants: "with a diagnosis of acute appendicitis". Children (< 15 years) and pregnant women were \\
& excluded. \\
& Gender: not given \\
& Age: not given \\
& Histology: $84 \%$ acutely inflamed \\
& LA: techniques "were not standardized"; four trocars were used in $81 \%$; stump closure with stapling de- \\
& vice in $80 \%$ ( $=93$ ) \\
& OA: through muscle-splitting right lower quadrant incision ( $\mathrm{n}=105)$ \\
& Antibiotics: cefoxitin $1 \mathrm{~g}$ IV
\end{tabular}

\section{Outcomes}

Hospital stay (total and postoperative); return to full activities (in subgroup only); operating time; complication rates; detailed cost analysis

Follow-up: until 1 year was complete in $98.5 \%$
No appendectomy: was performed in one OA participant with diverticulitis
Declaration of interest: not reported
Funding: not reported
Preoperative imaging: not reported
Analgesia requirements: "Morphine sulfate was administered intravenously by patient-controlled infu-
sion pump,...propoxyphene and acetaminophen orally,"
Sample size calculation: adequately reported

\section{Risk of bias}

\begin{tabular}{|c|c|c|}
\hline Bias & Authors' judgement & Support for judgement \\
\hline $\begin{array}{l}\text { Random sequence genera- } \\
\text { tion (selection bias) }\end{array}$ & Low risk & "were randomized by a random numbers table" \\
\hline $\begin{array}{l}\text { Allocation concealment } \\
\text { (selection bias) }\end{array}$ & Low risk & "sealed sequenced envelopes" \\
\hline $\begin{array}{l}\text { Blinding of participants } \\
\text { and personnel (perfor- } \\
\text { mance bias) } \\
\text { All outcomes }\end{array}$ & Unclear risk & Not reported \\
\hline $\begin{array}{l}\text { Blinding of outcome as- } \\
\text { sessment (detection bias) } \\
\text { All outcomes }\end{array}$ & Unclear risk & Not reported \\
\hline $\begin{array}{l}\text { Incomplete outcome data } \\
\text { (attrition bias) } \\
\text { All outcomes }\end{array}$ & Unclear risk & $\begin{array}{l}\text { "Complete follow up was obtained for } 195 \text { patients ( } 100 \% \text { open group, } 96,8 \% \\
\text { laparoscopic group)." Converted cases }(n=15,16 \%) \text { and those receiving other } \\
\text { surgery were analysed by ITT. }\end{array}$ \\
\hline $\begin{array}{l}\text { Selective reporting (re- } \\
\text { porting bias) }\end{array}$ & Unclear risk & No distinction between primary and secondary outcome \\
\hline
\end{tabular}


Long 2000 (Continued)
Other bias
Low risk
"All [trial surgeons] had well established laparoscopic practices."

Macarulla 1995

\begin{tabular}{ll}
\hline Methods & Randomised controlled trial \\
& Centres: one (Spain) \\
\hline Participants & Participants: "all patients above the age of 12 presenting with a preoperative diagnosis of acute appen- \\
& dicitis" \\
& Gender: $57 \%$ females \\
& Age: 26.5 and 28.8 years (mean) for LA and OA \\
& Histology: $91 \%$ inflamed.
\end{tabular}

Interventions

LAP: Appendix not removed if other diagnosis found on laparoscopy.

LA: three trocar technique $(12,10$, and $5 \mathrm{~mm})$; stump closure by endo loop (or stapler, $\mathrm{n}=2)(\mathrm{n}=106)$

OA: "through the classical right lower quadrant incision" (OA = 104)

Antibiotics: $1 \mathrm{mg} / \mathrm{kg}$ gentamicin IV and $500 \mathrm{mg}$ metronidazole IV

Outcomes Hospital stay; return to normal activities; complication rates; duration of operation; resumption of regular diet; costs (intra- and postoperative)

Follow-up: 30 days
No appendectomy: appendix left in situ in 4 cases
Declaration of interest: not reported
Funding: not reported
Preoperative imaging: not reported
Analgesia requirements: NSAIDs: parenteral every $4 \mathrm{~h}$ as needed, oral every $8 \mathrm{~h}$ as needed; consump-
tion of parenteral and oral analgesia significantly lower in laparoscopic group
Sample size calculation: not reported

\section{Risk of bias}

\begin{tabular}{lll}
\hline Bias & Authors' judgement & Support for judgement \\
\hline $\begin{array}{l}\text { Random sequence genera- } \\
\text { tion (selection bias) }\end{array}$ & Unclear risk & "Randomization was determined..." \\
\hline $\begin{array}{l}\text { Allocation concealment } \\
\text { (selection bias) }\end{array}$ & Low risk & "... by the envelope method." \\
\hline $\begin{array}{l}\text { Blinding of participants } \\
\begin{array}{l}\text { and personnel (perfor- } \\
\text { mance bias) }\end{array}\end{array}$ & Unclear risk & Not reported \\
All outcomes & \\
\hline
\end{tabular}

\begin{tabular}{ll}
\hline Blinding of outcome as- & Unclear risk \\
sessment (detection bias) & \\
All outcomes & \\
\hline
\end{tabular}

Incomplete outcome data Low risk No losses to follow-up visible. Conversions $(n=9,9 \%)$ were analysed by ITT.


Macarulla 1995 (Continued)

All outcomes

Selective reporting (re- $\quad$ Unclear risk $\quad$ No distinction between primary and secondary outcome
porting bias)

\begin{tabular}{ll}
\hline Other bias $\quad$ High risk & $\begin{array}{l}\text { Surgeons were described as "experienced", but "all conversions occurred in } \\
\text { the first } 50 \text { cases." }\end{array}$
\end{tabular}

\section{Mahmood 2016}

\begin{tabular}{|c|c|}
\hline Methods & $\begin{array}{l}\text { Randomised controlled trial } \\
\text { Centres: one (Pakistan) }\end{array}$ \\
\hline Participants & $\begin{array}{l}\text { Participants: "...diagnosed with acute appendicitis were included in the study. Diagnosis of acute ap- } \\
\text { pendicitis was confirmed by history, physical examination, complete blood picture, urinalysis, and ul- } \\
\text { trasound of abdomen/pelvis." } \\
\text { Gender: } 45 \% \text { females } \\
\text { Age: } 21.0 \text { and } 23.49 \text { years (mean) for LA and OA } \\
\text { Histology: not reported }\end{array}$ \\
\hline
\end{tabular}

\begin{tabular}{|c|c|}
\hline Interventions & $\begin{array}{l}\text { LA: three trocar technique }(5,5 \text {, and } 10 \mathrm{~mm}) \text {, stump closure technique not described ( } \mathrm{n}=100) \\
\text { OA: "...an incision was given in the right iliac fossa and after opening the abdominal muscle layers," (OA } \\
=100) \\
\text { Antibiotics: "All patients received a single dose of antibiotic preoperatively followed by two doses in } \\
\text { post op period." }\end{array}$ \\
\hline Outcomes & Complication rates \\
\hline \multirow[t]{6}{*}{ Notes } & $\begin{array}{l}\text { Follow-up: } 14 \text { days } \\
\text { No appendectomy: none reported }\end{array}$ \\
\hline & Declaration of interest: not reported \\
\hline & Funding: not reported \\
\hline & Preoperative imaging: "...ultrasound of abdomen/pelvis." \\
\hline & Analgesia requirements: not reported \\
\hline & Sample size calculation: not reported \\
\hline
\end{tabular}

\section{Risk of bias}

Bias Authors' judgement Support for judgement

Random sequence genera- Unclear risk "Patients were admitted through emergency department, written informed tion (selection bias) consent was taken and they were randomly allocated to two groups."

\begin{tabular}{lll}
$\begin{array}{l}\text { Allocation concealment } \\
\text { (selection bias) }\end{array}$ & Unclear risk & Not reported \\
\hline $\begin{array}{l}\text { Blinding of participants } \\
\text { and personnel (perfor- } \\
\text { mance bias) }\end{array}$ & Unclear risk & Not reported \\
All outcomes & \\
\hline
\end{tabular}


Mahmood 2016 (Continued)
Blinding of outcome as-
Unclear risk
Not reported sessment (detection bias)

All outcomes

\begin{tabular}{lll}
\hline $\begin{array}{l}\text { Incomplete outcome data } \\
\text { (attrition bias) } \\
\text { All outcomes }\end{array}$ & Low risk & $\begin{array}{l}\text { No losses from follow-up visible. No conversions or other changes in surgical } \\
\text { procedure }\end{array}$ \\
\hline $\begin{array}{l}\text { Selective reporting (re- } \\
\text { porting bias) }\end{array}$ & Unclear risk & No distinction between primary and secondary outcome \\
\hline $\begin{array}{l}\text { Other bias } \\
\text { Low risk }\end{array}$ & $\begin{array}{l}\text { "All procedures were performed by consultant surgeons well experienced in } \\
\text { the procedures to avoid bias." }\end{array}$ \\
\hline
\end{tabular}

\section{Martin 1995}

\begin{tabular}{|c|c|}
\hline Methods & $\begin{array}{l}\text { Randomised controlled trial } \\
\text { Centres: one (USA) }\end{array}$ \\
\hline Participants & $\begin{array}{l}\text { Participants: "adult patients with the presumptive diagnosis of acute appendicitis" } \\
\text { Gender: } 41 \% \text { females } \\
\text { Age: } 27 \text { and } 29 \text { years (mean) in LA and OA } \\
\text { Histology: } 86 \% \text { inflamed }\end{array}$ \\
\hline Interventions & $\begin{array}{l}\text { LA: three trocar technique }(12,10 \text {, and } 5 \mathrm{~mm}) \text {; stump closure by stapling device }(\mathrm{n}=88) \\
\text { OA: via McBurney or Rockey-Davis right lower quadrant muscle splitting incision }(n=81) \\
\text { Antibiotics: } 1 \text { g ceftriaxone }\end{array}$ \\
\hline Outcomes & Hospital stay; return to normal activities; complication rates; duration of operation \\
\hline \multirow[t]{7}{*}{ Notes } & $\begin{array}{l}\text { Follow-up: Participants "were questioned during follow-up visits, by telephone, and by mailed ques- } \\
\text { tionnaire." The duration of follow-up was apparently about a month or until return to normal activities. } \\
\text { No appendectomy: not reported }\end{array}$ \\
\hline & Declaration of interest: not reported \\
\hline & Funding: not reported \\
\hline & Preoperative imaging: not reported \\
\hline & Analgesia requirements: not reported \\
\hline & Sample size calculation: not reported \\
\hline & $\begin{array}{l}\text { Comment: Data were given without measure of dispersion. In Table 1, columns were incorrectly la- } \\
\text { belled. }\end{array}$ \\
\hline
\end{tabular}

\section{Risk of bias}

\begin{tabular}{lll}
\hline Bias & Authors' judgement & Support for judgement \\
\hline $\begin{array}{l}\text { Random sequence genera- } \\
\text { tion (selection bias) }\end{array}$ & Unclear risk & "patients were randomised" \\
\hline $\begin{array}{l}\text { Allocation concealment } \\
\text { (selection bias) }\end{array}$ & Unclear risk & Not reported \\
\hline
\end{tabular}


Martin 1995 (Continued)

Blinding of participants Unclear risk Not reported
and personnel (perfor-
mance bias)
All outcomes

Alloutcomes

\begin{tabular}{lll}
\hline Blinding of outcome as- & Unclear risk & Not reported \\
sessment (detection bias) &
\end{tabular}

All outcomes

Incomplete outcome data Low risk (attrition bias)

All outcomes

\begin{tabular}{lll}
\hline $\begin{array}{l}\text { Selective reporting (re- } \\
\text { porting bias) }\end{array}$ & Unclear risk & No distinction between primary and secondary outcome \\
\hline Other bias & Unclear risk & $\begin{array}{l}\text { "All operations were performed by surgical residents with the assistance of the } \\
\text { attending surgeon". }\end{array}$
\end{tabular}

\section{Minné 1997}

\begin{tabular}{|c|c|}
\hline Methods & $\begin{array}{l}\text { Randomised controlled trial } \\
\text { Centres: one (USA) }\end{array}$ \\
\hline Participants & $\begin{array}{l}\text { Participants: patients aged >= } 12 \text { yr with a presumptive diagnosis of acute appendicitis. Patients with a } \\
\text { lower midline scar and pregnant women were excluded. } \\
\text { Gender: } 38 \% \text { females } \\
\text { Age: } 31.5 \text { and } 31.1 \text { years (median) in LA and OA } \\
\text { Histology: } 86 \% \text { inflamed }\end{array}$ \\
\hline Interventions & $\begin{array}{l}\text { LA: "using a standardized } 3 \text { or } 4 \text { trocar approach (umbilical, } 10-12 \mathrm{~mm} \text {; suprapubic, } 10-12 \mathrm{~mm} \text {; right up- } \\
\text { per quadrant, } 5 \mathrm{~mm} \text {; and right lower quadrant, } 5 \mathrm{~mm} \text {, optional)"; stump closure by stapler or loops ( }= \\
27 \text { ) } \\
\text { OA: via McBurney or Rockey-Davis right lower quadrant muscle splitting incision ( } \mathrm{n}=23 \text { ) } \\
\text { Antibiotics: } 1 \text { g cefotetan }\end{array}$ \\
\hline Outcomes & Hospital stay; return to normal activities; complication rates; duration of operation; pain (VAS); costs \\
\hline Notes & $\begin{array}{l}\text { Follow-up: "by follow-up visits or by telephone"; time interval not mentioned } \\
\text { No appendectomy: not reported } \\
\text { Declaration of interest: not reported } \\
\text { Funding: not reported } \\
\text { Preoperative imaging: not reported } \\
\text { Analgesia requirements: not reported } \\
\text { Sample size calculation: not reported }\end{array}$ \\
\hline
\end{tabular}

\section{Risk of bias}


Minné 1997 (Continued)

$\begin{array}{ll}\begin{array}{l}\text { Random sequence genera- } \\ \text { tion (selection bias) }\end{array} & \begin{array}{l}\text { "Randomization was done according to a master list consisting of a repeating } \\ \text { sequence of } 10 \text { random numbers" }\end{array}\end{array}$
tion (selection bias) sequence of 10 random numbers"

\begin{tabular}{lll}
\hline $\begin{array}{l}\text { Allocation concealment } \\
\text { (selection bias) }\end{array}$ & Unclear risk & Not reported \\
\hline $\begin{array}{l}\text { Blinding of participants } \\
\text { and personnel (perfor- } \\
\text { mance bias) }\end{array}$ & Unclear risk & Not reported \\
All outcomes & &
\end{tabular}

Blinding of outcome as- Unclear risk Not reported
sessment (detection bias) All outcomes

\section{Incomplete outcome data High risk} (attrition bias)

Conversions (7\%) were analysed according to ITT, but 7 out of 57 initially ranAll outcomes domised participants were excluded from analyses due to protocol violation (unallowed analgesia regimen or midline incision). It was not clear, to which group these excluded cases belonged. All remaining participants completed the study, including postdischarge follow-up.

Selective reporting (re- Unclear risk $\quad$ No distinction between primary and secondary outcome
porting bias)

Other bias Low risk All operators "were experienced laparoscopic surgeons".

\section{Moberg 2005}

\begin{tabular}{ll}
\hline Methods & Randomised controlled trial \\
& Centre: one (Sweden) \\
\hline Participants & Participants:over 15 years of age with clinical suspicion of acute appendicitis and a laparoscopic diag- \\
& nosis of acute appendicitis (i.e. randomisation took place after diagnostic laparoscopy) \\
& Gender: $36 \%$ females \\
& Age: 31 years (median) in both groups \\
& Histology: $100 \%$ inflamed
\end{tabular}

Interventions LA: three trocar technique $(12,10$, and $5 \mathrm{~mm})$, "the mesoappendix was dissected using electrocautery or an ultrasound dissector, and the appendix was removed using a linear cutting device." $(n=81)$ OA: muscle-splitting incision $(n=82)$

Antibiotics: cefuroxime and metronidazole (dose not stated)

\begin{tabular}{|c|c|}
\hline Outcomes & Time to full recovery; operating time; complications; hospital stay; functional status \\
\hline \multirow[t]{5}{*}{ Notes } & $\begin{array}{l}\text { Follow-up: "An independent observer (study nurse) examined each patient 7-10 days after surgery". "A } \\
\text { final check-up was performed } 3 \text { months after operation." }\end{array}$ \\
\hline & No appendectomy: not reported \\
\hline & Declaration of interest: not reported \\
\hline & $\begin{array}{l}\text { Funding: "This study was supported financially by the Einar and Inga Nilsson Foundation for Surgical } \\
\text { Research, Craffoord Foundation and FoU, the research unit of Skane." }\end{array}$ \\
\hline & Preoperative imaging: not reported \\
\hline
\end{tabular}


Moberg 2005 (Continued)

Analgesia requirements: not reported

Sample size calculation: adequately reported

\title{
Risk of bias
}

\begin{tabular}{lll}
\hline Bias & Authors' judgement & Support for judgement \\
\hline $\begin{array}{l}\text { Random sequence genera- } \\
\text { tion (selection bias) }\end{array}$ & Low risk & "Randomization was performed in blocks of 20 computer-generated,..." \\
\hline $\begin{array}{l}\text { Allocation concealment } \\
\text { (selection bias) }\end{array}$ & Low risk & "... closed, numbered envelopes, drawn in sequential order." \\
\hline $\begin{array}{l}\text { Blinding of participants } \\
\text { and personnel (perfor- } \\
\text { mance bias) } \\
\text { All outcomes }\end{array}$ & Low risk & $\begin{array}{l}\text { "Four identical wound dressings were applied regardless of the type of } \\
\text { surgery. Blood was applied to all four dressings to minimize the chance of the } \\
\text { type of operation becoming known during the postoperative period. The oper- } \\
\text { ative technique was unknown to the patient, the staff on the surgical ward and } \\
\text { the study nurse until after the first postoperative examination, } 7-10 \text { days after } \\
\text { appendicectomy." "The patient was blinded if the dressings needed changing } \\
\text { during the hospital stay." }\end{array}$
\end{tabular}

Blinding of outcome as- Low risk sessment (detection bias)

All outcomes

\begin{abstract}
"Four identical wound dressings were applied regardless of the type of surgery. Blood was applied to all four dressings to minimize the chance of the type of operation becoming known during the postoperative period. The operative technique was unknown to the patient, the staff on the surgical ward and the study nurse until after the first postoperative examination, 7-10 days after appendicectomy." "The patient was blinded if the dressings needed changing during the hospital stay."
\end{abstract}

\begin{tabular}{ll}
\hline $\begin{array}{l}\text { Incomplete outcome data } \\
\text { (attrition bias) }\end{array}$ & Low risk \\
All & were correctly analysed in the LA group.
\end{tabular}

All outcomes

\begin{tabular}{lll}
\hline $\begin{array}{l}\text { Selective reporting (re- } \\
\text { porting bias) }\end{array}$ & Low risk & The primary outcome (time to full recovery) was adequately described. \\
\hline Other bias & Low risk & Operations were done by "experienced surgeons in an educational setting." \\
\hline
\end{tabular}

\section{Moirangthem 2008}

\begin{tabular}{ll}
\hline Methods & Randomised controlled trial \\
& Centre: one (India) \\
\hline Participants & Participants: patients with clinical diagnosis of acute appendicitis \\
& Gender: $54 \%$ females \\
& Age: 31.1 and 35.1 years (mean) in LA and OA \\
& Histology: not reported \\
\hline Interventions & LA: using three ports (10, 10, and 5 mm), "the appendix was ligated with 2-0 Vicryl without invagination \\
of the stump" ( $\mathrm{n}=25)$ \\
OA: McBurney's muscle splitting incision ( $\mathrm{n}=25)$ \\
Antibiotics: cephalosporins and metronidazole in complicated cases
\end{tabular}


Moirangthem 2008 (Continued)

Outcomes

Operating time; hospital stay; wound infection, time until reintroduction of solid diet; total number of postoperative parenteral doses of analgesic

Notes

Follow-up: not reported

No appendectomy: not reported

Declaration of interest: not reported

Funding: not reported

Preoperative imaging: not reported

Analgesia requirements: "... diclofenac sodium were given for $24 \mathrm{hrs}$. Further analgesics were given based on the participant's perception of pain." "... parenteral doses of analgesic received was significantly reduced in the laparoscopic group $(P<0.01)$."

Sample size calculation: not reported

\section{Risk of bias}

\begin{tabular}{|c|c|c|}
\hline Bias & Authors' judgement & Support for judgement \\
\hline $\begin{array}{l}\text { Random sequence genera- } \\
\text { tion (selection bias) }\end{array}$ & Unclear risk & "Patients were randomized prospectively to either LA or OA." \\
\hline $\begin{array}{l}\text { Allocation concealment } \\
\text { (selection bias) }\end{array}$ & Unclear risk & Not reported \\
\hline $\begin{array}{l}\text { Blinding of participants } \\
\text { and personnel (perfor- } \\
\text { mance bias) } \\
\text { All outcomes }\end{array}$ & Unclear risk & Not reported \\
\hline $\begin{array}{l}\text { Blinding of outcome as- } \\
\text { sessment (detection bias) } \\
\text { All outcomes }\end{array}$ & Unclear risk & Not reported \\
\hline $\begin{array}{l}\text { Incomplete outcome data } \\
\text { (attrition bias) } \\
\text { All outcomes }\end{array}$ & Low risk & $\begin{array}{l}\text { Data appeared to be complete. No conversions or other changes in surgical } \\
\text { procedure }\end{array}$ \\
\hline $\begin{array}{l}\text { Selective reporting (re- } \\
\text { porting bias) }\end{array}$ & Unclear risk & No distinction between primary and secondary outcome \\
\hline Other bias & Unclear risk & The surgeon's skills were not described. \\
\hline
\end{tabular}

\section{Mutter 1996}

\begin{tabular}{ll}
\hline Methods & Randomised controlled trial \\
& Centres: one (?) (France) \\
\hline Participants & Participants: men aged 16 to 65 yr with symptoms and signs suggestive of acute appendicitis. Patients \\
& with signs of generalised peritonitis or uncertain preoperative diagnosis were excluded \\
& Gender: only males \\
& Age: 28.6 and 26.8 years (mean) in LA and OA \\
& Histology: $94 \%$ inflamed \\
\hline
\end{tabular}


Mutter 1996 (Continued)

Interventions
LA: three trocars $(10,10$, and $5 \mathrm{~mm})$; stump closure by loops (except one case where a stapler was used) $(n=50)$

OA: via muscle-splitting approach $(n=50)$

Antibiotics: cefuroxime $1.5 \mathrm{mg}$ IV

\begin{tabular}{|c|c|}
\hline Outcomes & Hospital stay; return to normal activities; complication rates; duration of operation; pain (VAS); costs \\
\hline \multirow[t]{6}{*}{ Notes } & $\begin{array}{l}\text { Follow-up: "The wounds were inspected on postoperative day } 8 \text { ". } \\
\text { No appendectomy: not reported }\end{array}$ \\
\hline & Declaration of interest: not reported \\
\hline & Funding: not reported \\
\hline & Preoperative imaging: not reported \\
\hline & Analgesia requirements: not reported \\
\hline & Sample size calculation: not reported \\
\hline
\end{tabular}

\section{Risk of bias}

\section{Bias}

Random sequence genera- Unclear risk tion (selection bias)

\begin{tabular}{lll}
\hline $\begin{array}{l}\text { Allocation concealment } \\
\text { (selection bias) }\end{array}$ & Unclear risk & Not reported \\
\hline
\end{tabular}

Blinding of participants $\quad$ Unclear risk
and personnel (perfor-

\begin{tabular}{|c|c|c|}
\hline $\begin{array}{l}\text { Blinding of outcome as- } \\
\text { sessment (detection bias) } \\
\text { All outcomes }\end{array}$ & Unclear risk & Not reported \\
\hline $\begin{array}{l}\text { Incomplete outcome data } \\
\text { (attrition bias) } \\
\text { All outcomes }\end{array}$ & Low risk & $\begin{array}{l}\text { Data on main results appeared to be complete. Converted cases }(n=6,12 \%) \\
\text { were analysed by ITT. }\end{array}$ \\
\hline $\begin{array}{l}\text { Selective reporting (re- } \\
\text { porting bias) }\end{array}$ & Unclear risk & No distinction between primary and secondary outcome \\
\hline Other bias & Low risk & Surgeons were "skilled in both open and laparoscopic surgery". \\
\hline
\end{tabular}

\section{Navarra 2000}

\begin{tabular}{ll}
\hline Methods & Randomised controlled trial \\
& Centres: one (Italy) \\
\hline Participants & Participants: female patients with right iliac fossa pain \\
& Gender: $100 \%$ females \\
& Age: 26,3 and 29.6 years (mean) in LA and OA
\end{tabular}


Navarra 2000 (Continued)

Histology: unclear data

\section{Risk of bias}

\begin{tabular}{|c|c|c|}
\hline Bias & Authors' judgement & Support for judgement \\
\hline $\begin{array}{l}\text { Random sequence genera- } \\
\text { tion (selection bias) }\end{array}$ & Low risk & using "a list of random codes",... \\
\hline $\begin{array}{l}\text { Allocation concealment } \\
\text { (selection bias) }\end{array}$ & Low risk & which was kept "in an envelope" by a surgeon uninvolved in the trial \\
\hline $\begin{array}{l}\text { Blinding of participants } \\
\text { and personnel (perfor- } \\
\text { mance bias) } \\
\text { All outcomes }\end{array}$ & Unclear risk & Not reported \\
\hline $\begin{array}{l}\text { Blinding of outcome as- } \\
\text { sessment (detection bias) } \\
\text { All outcomes }\end{array}$ & Unclear risk & Not reported \\
\hline $\begin{array}{l}\text { Incomplete outcome data } \\
\text { (attrition bias) } \\
\text { All outcomes }\end{array}$ & Low risk & $\begin{array}{l}\text { Additional data on the } 2 \text { converted cases were obtained from the authors, thus } \\
\text { allowing ITT analysis. Cases, in whom no appendectomy was necessary, were } \\
\text { analysed according to ITT. }\end{array}$ \\
\hline $\begin{array}{l}\text { Selective reporting (re- } \\
\text { porting bias) }\end{array}$ & Unclear risk & No distinction between primary and secondary outcome \\
\hline Other bias & Unclear risk & The surgeon's skills were not described. \\
\hline
\end{tabular}

Nordentoft 2000

\begin{tabular}{ll}
\hline Methods & Randomised controlled trial \\
& Centres: one (Denmark) \\
\hline Participants & Participants: with suspected acute appendicitis \\
& Gender: not given \\
\hline
\end{tabular}


Nordentoft 2000 (Continued)

Age: not given

Histology: not given

\begin{tabular}{ll}
\hline Interventions & $\begin{array}{l}\text { LAP: appendix left in situ, if found to be normal. LA: three trocar technique; stump closure by endo-sta- } \\
\text { pler }(\mathrm{n}=12) \\
\text { OA: via transverse incision }(\mathrm{n}=11) \\
\text { Antibiotics: Not given }\end{array}$ \\
\hline Outcomes & Duration of operation; rates of infected blood cultures \\
\hline Notes & $\begin{array}{l}\text { Follow-up: not reported } \\
\text { No appendectomy: unclear data, participants were excluded }\end{array}$ \\
& Declaration of interest: not reported \\
& Funding: not reported \\
& Preoperative imaging: not reported \\
Analgesia requirements: not reported & Sample size calculation: not reported
\end{tabular}

\section{Risk of bias}

\begin{tabular}{lll}
\hline Bias & Authors' judgement & Support for judgement \\
\hline $\begin{array}{l}\text { Random sequence genera- } \\
\text { tion (selection bias) }\end{array}$ & Unclear risk & "Patients were randomised..." \\
\hline $\begin{array}{l}\text { Allocation concealment } \\
\text { (selection bias) }\end{array}$ & Unclear risk & Not reported \\
\hline $\begin{array}{l}\text { Blinding of participants } \\
\text { and personnel (perfor- } \\
\text { mance bias) } \\
\text { All outcomes }\end{array}$ & Unclear risk & Not reported \\
\hline
\end{tabular}

\begin{tabular}{lll}
$\begin{array}{l}\text { Blinding of outcome as- } \\
\text { sessment (detection bias) } \\
\text { All outcomes }\end{array}$ & Unclear risk & Not reported \\
\hline $\begin{array}{l}\text { Incomplete outcome data } \\
\begin{array}{l}\text { (attrition bias) } \\
\text { All outcomes }\end{array}\end{array}$ & High risk & $\begin{array}{l}\text { In total, } 7 \text { of } 30 \text { randomised participants were excluded from analyses due to } \\
\text { protocol violations. One converted case and several participants with a nor- } \\
\text { mal appendix (on laparoscopy or histology) were excluded from the analysis. }\end{array}$
\end{tabular}

\begin{tabular}{lll}
\hline $\begin{array}{l}\text { Selective reporting (re- } \\
\text { porting bias) }\end{array}$ & Unclear risk & No distinction between primary and secondary outcome \\
\hline Other bias & Unclear risk & The surgeon's skills were not described. \\
\hline
\end{tabular}

\section{Olsen 1993}

\begin{tabular}{ll}
\hline Methods & Randomised controlled trial \\
& Centres: one (Denmark) \\
\hline Participants & Participants: women aged 15-56 years with clinical signs of acute appendicitis
\end{tabular}


Olsen 1993 (Continued)

Gender: all females

Age: 25.3 and 25.8 years (mean) in LA and OA

Histology: 52\% inflamed

Interventions

LAP: Diagnostic laparoscopy followed by OA when acute appendicitis was visible or no other diagnosis was found $(n=30)$

OPEN: via transverse incision in right iliac fossa $(n=30)$

Antibiotics: not reported

\begin{tabular}{|c|c|}
\hline Outcomes & $\begin{array}{l}\text { Rates of unnecessary appendectomies and participants without diagnosis established; complications; } \\
\text { hospital stay }\end{array}$ \\
\hline \multirow[t]{6}{*}{ Notes } & $\begin{array}{l}\text { Follow-up: "late follow-up was not performed" } \\
\text { No appendectomy: not reported }\end{array}$ \\
\hline & Declaration of interest: not reported \\
\hline & Funding: not reported \\
\hline & Preoperative imaging: not reported \\
\hline & Analgesia requirements: not reported \\
\hline & Sample size calculation: not reported \\
\hline
\end{tabular}

\section{Risk of bias}

Bias Authors' judgement Support for judgement

Random sequence genera- Unclear risk "The trial was performed in a randomized fashion".

tion (selection bias)

\begin{tabular}{lll}
\hline $\begin{array}{l}\text { Allocation concealment } \\
\text { (selection bias) }\end{array}$ & Unclear risk & Not reported \\
\hline $\begin{array}{l}\text { Blinding of participants } \\
\text { and personnel (perfor- } \\
\text { mance bias) }\end{array}$ & Unclear risk & Not reported \\
All outcomes & \\
\hline
\end{tabular}

$\begin{array}{ll}\text { Blinding of outcome as- } & \text { Unclear risk }\end{array}$

All outcomes

Incomplete outcome data Low risk After randomisation, not a single participant was excluded from analysis.
(attrition bias)

All outcomes

Selective reporting (re- Unclear risk $\quad$ No distinction between primary and secondary outcome
porting bias)

\begin{tabular}{ll}
\hline Other bias $\quad$ Low risk $\quad$ Surgeons had "laparoscopic skills". \\
\hline
\end{tabular}

Ortega 1996

Methods Randomised controlled trial


Ortega 1996 (Continued)

Centres: 10 centres (USA)

Comment: The study was three-armed, as it compared two laparoscopic techniques versus open surgery.

Participants: patients with a clinical diagnosis of acute, chronic, or perforated appendicitis. Pregnant
women were excluded.
Gender: $29 \%$ female
Age: 25 years (mean)
Histology: $84 \%$ inflamed

\begin{tabular}{ll}
\hline Interventions & LA: three trocar technique; stump closure by ligature or stapler $(n=89+n=78)$ \\
& $\begin{array}{l}\text { OA: via "transverse muscle-splitting incision }(5 \text { to } 6 \mathrm{~cm}) "(n=86) \\
\text { Antibiotics: not reported }\end{array}$ \\
\hline Outcomes & $\begin{array}{l}\text { Hospital stay; return to normal activities; complication rates; duration of operation; pain (VAS + anal- } \\
\text { gesic dosages) }\end{array}$
\end{tabular}

Notes $\quad$ Follow-up: "patients came back for their postoperative visit"; no time point given No appendectomy: not reported

Declaration of interest: not reported

Funding: "This study was supported with a grant from the U.S. Surgical Corporation, Norwalk, Connecticut."

Preoperative imaging: not reported

Analgesia requirements: "A pain score of 4 received an intramuscular injection of meperidine; 3 elicited two acetaminophen with codeine tablets, 2 was given one tablet of acetaminophen with codeine; and 1 got plain acetaminophen." "The mean number of days for which patients required pain medication..." [...was similar between the groups $(P=N S)$.]

Sample size calculation: not reported

\section{Risk of bias}

\begin{tabular}{|c|c|c|}
\hline Bias & Authors' judgement & Support for judgement \\
\hline $\begin{array}{l}\text { Random sequence genera- } \\
\text { tion (selection bias) }\end{array}$ & Low risk & $\begin{array}{l}\text { "Randomization was executed by a computer-generated random numbers ta- } \\
\text { ble..." }\end{array}$ \\
\hline $\begin{array}{l}\text { Allocation concealment } \\
\text { (selection bias) }\end{array}$ & Low risk & $\begin{array}{l}\text { "...administered centrally via a toll-free telephone connection on a } 24 \text {-hour ba- } \\
\text { sis." }\end{array}$ \\
\hline $\begin{array}{l}\text { Blinding of participants } \\
\text { and personnel (perfor- } \\
\text { mance bias) } \\
\text { All outcomes }\end{array}$ & Low risk & $\begin{array}{l}\text { In a subgroup where pain was measured "the patients' abdominal wounds } \\
\text { were concealed by two army battle dressings applied at the conclusion of } \\
\text { surgery." }\end{array}$ \\
\hline $\begin{array}{l}\text { Blinding of outcome as- } \\
\text { sessment (detection bias) } \\
\text { All outcomes }\end{array}$ & Low risk & The nurse, who measured pain with the VAS was also blinded. \\
\hline $\begin{array}{l}\text { Incomplete outcome data } \\
\text { (attrition bias) } \\
\text { All outcomes }\end{array}$ & Low risk & $\begin{array}{l}\text { For most outcomes, no exclusions or losses to follow-up were reported. Con- } \\
\text { verted cases }(n=11,7 \%) \text { were analysed by ITT. However, data analysis on hos- } \\
\text { pital stay excluded } 5 \text { participants, who stayed longer than } 9 \text { days. }\end{array}$ \\
\hline $\begin{array}{l}\text { Selective reporting (re- } \\
\text { porting bias) }\end{array}$ & Unclear risk & No distinction between primary and secondary outcome \\
\hline
\end{tabular}


Ortega 1996 (Continued)

$\begin{array}{ll}\text { Other bias } \quad \text { Low risk } & \begin{array}{l}\text { Operations were performed by "residents with attending surgeons experi- } \\ \text { enced in laparoscopic and open surgical techniques". }\end{array}\end{array}$

enced in laparoscopic and open surgical techniques".

Pedersen 2001

Methods Randomised controlled trial

Centres: three departments in two university hospitals (Denmark)

\begin{tabular}{ll}
\hline Participants & Participants: "patients with a clinical diagnosis of acute appendicitis"; children were included at one of \\
& the three centres \\
& Gender: $53 \%$ females \\
Age: 26 and 27 years (median) in LA and OA \\
Histology: $79 \%$ inflamed
\end{tabular}

\begin{tabular}{ll}
\hline Interventions & $\begin{array}{l}\text { LA: three trocar technique }(10,10, \text { and } 5 \mathrm{~mm}) \text {; stump closure by ligature }(\mathrm{n}=282) \\
\text { OA: via muscle-splitting incision }(\mathrm{n}=301) \\
\text { Antibiotics: only given in perforated or gangrenous cases }\end{array}$ \\
\hline Outcomes & $\begin{array}{l}\text { Hospital stay; return to normal activities; return to work; complication rates; duration of operation; } \\
\text { cosmesis (VAS) }\end{array}$ \\
\hline Fotes & Follow-up: $80.3 \%$ of participants were seen in the outpatient clinic four weeks after discharge. \\
& Declaration of interest: not reported \\
& Funding: "Jacob and Olga Madsen's Fund provided financial support." \\
& Preoperative imaging: not reported \\
& Analgesia requirements: not reported \\
& Sample size calculation: not reported
\end{tabular}

\section{Risk of bias}

\begin{tabular}{lll}
\hline Bias & Authors' judgement & Support for judgement \\
\hline $\begin{array}{l}\text { Random sequence genera- } \\
\text { tion (selection bias) }\end{array}$ & Low risk & $\begin{array}{l}\text { "using computer-generated, numbered,..." } \\
\text { Randomisation was stratified for age. }\end{array}$ \\
\hline $\begin{array}{ll}\text { Allocation concealment } \\
\text { (selection bias) }\end{array}$ & Low risk & "...opaque and sealed envelopes" \\
\hline $\begin{array}{l}\text { Blinding of participants } \\
\text { and personnel (perfor- } \\
\text { mance bias) }\end{array}$ & Unclear risk & Not reported \\
All outcomes & \\
\hline
\end{tabular}

\begin{tabular}{lll}
\hline $\begin{array}{l}\text { Blinding of outcome as- } \\
\text { sessment (detection bias) } \\
\text { All outcomes }\end{array}$ & Unclear risk & Not reported \\
\hline $\begin{array}{l}\text { Incomplete outcome data } \\
\text { (attrition bias) }\end{array}$ & Unclear risk & $\begin{array}{l}\text { Converted cases }(\mathrm{n}=65 ; 23 \%) \text { were analysed according to ITT. For most out- } \\
\text { All outcomes }\end{array}$ \\
\end{tabular}


Pedersen 2001 (Continued)

ble in $80.3 \%$ of participants, but no information was provided on whether the completeness of follow-up differed between the groups.

\begin{tabular}{lll}
\hline $\begin{array}{l}\text { Selective reporting (re- } \\
\text { porting bias) }\end{array}$ & Low risk & Primary outcome (hospital stay) was adequately described. \\
\hline Other bias & Low risk & $\begin{array}{l}\text { Trial surgeons were "of comparable experience" and were "equally divided be- } \\
\text { tween registrars and senior registrars". }\end{array}$ \\
\hline
\end{tabular}

Perner 1999

\begin{tabular}{ll}
\hline Methods & Randomised controlled trial \\
& Centres: one (Denmark) \\
\hline Participants & $\begin{array}{l}\text { Participants: with suspected appendicitis } \\
\text { Gender: } 73 \% \text { females } \\
\text { Age: } 33 \text { years median } \\
\text { Histology: } 64 \% \text { inflamed }\end{array}$ \\
\hline Interventions & LA: not described ( $=7)$ \\
& Antibiotics: not reported \\
\hline Outcomes & Plasma potassium concentration; anaesthesia time \\
\hline Notes & Follow-up: not reported \\
No appendectomy: probably 4 participants & Declaration of interest: not reported \\
& Funding: not reported \\
Preoperative imaging: not reported \\
Analgesia requirements: not reported \\
Sample size calculation: not reported
\end{tabular}

\section{Risk of bias}

\section{Bias}

Random sequence genera- Unclear risk tion (selection bias)

\begin{tabular}{l}
$\begin{array}{l}\text { Allocation concealment } \\
\text { (selection bias) }\end{array}$ \\
\hline
\end{tabular}

\section{Authors' judgement Support for judgement}

"Patients were randomised..." Not reported

Blinding of participants and personnel (performance bias)

All outcomes 
Perner 1999 (Continued)

Incomplete outcome data Unclear risk No reporting of the number and reasons for exclusions or completeness of fol(attrition bias)

All outcomes

\begin{tabular}{lll}
$\begin{array}{l}\text { Selective reporting (re- } \\
\text { porting bias) }\end{array}$ & Unclear risk & No distinction between primary and secondary outcome \\
\hline Other bias & Unclear risk & The surgeon's skills were not described.
\end{tabular}

\section{Pozo 1996}

\begin{tabular}{|c|c|}
\hline Methods & $\begin{array}{l}\text { Randomised controlled trial } \\
\text { Centres: one (Venezuela) }\end{array}$ \\
\hline Participants & $\begin{array}{l}\text { Participants: with a clinical diagnosis of acute appendicitis ("con el diagnóstico clínico de apendicitis } \\
\text { aguda") } \\
\text { Gender: } 48 \% \text { females } \\
\text { Age: } 22.2 \text { and } 22.1 \text { years (mean) in LA and OA } \\
\text { Histology: } 38(86 \%) \text { inflamed }\end{array}$ \\
\hline Interventions & $\begin{array}{l}\text { LA: three trocar technique }(12,10 \text {, and } 10 \mathrm{~mm}) \text { with a forth trocar }(5 \mathrm{~mm}) \text { in some participants; stump } \\
\text { closure by } 3 \text { Roeder loops }(\mathrm{n}=20) \\
\text { OA: via Rockey-Davis incision }(\mathrm{n}=24) \\
\text { Antibiotics: All participants received clindamycin and an aminoglycoside. Antibiotics were continued } \\
\text { in gangrenous and severely inflamed cases. }\end{array}$ \\
\hline
\end{tabular}

Outcomes

Hospital stay; return to normal activities; complication rates; duration of operation; return of bowel function

\begin{tabular}{|c|c|c|}
\hline Notes & $\begin{array}{l}\text { Follow-up: on day } 7 \text { a } \\
\text { No appendectomy: no } \\
\text { Declaration of interes } \\
\text { Funding: not reported } \\
\text { Preoperative imaging } \\
\text { Analgesia requiremen } \\
\text { Sample size calculatic }\end{array}$ & $\begin{array}{l}21 \text { after appendectomy } \\
\text { reported } \\
\text { not reported } \\
\text { ot reported } \\
\text { not reported } \\
\text { not reported }\end{array}$ \\
\hline \multicolumn{3}{|l|}{ Risk of bias } \\
\hline Bias & Authors' judgement & Support for judgement \\
\hline $\begin{array}{l}\text { Random sequence genera- } \\
\text { tion (selection bias) }\end{array}$ & Unclear risk & $\begin{array}{l}\text { The allocation of participants into one or the other group was random. ("La in- } \\
\text { clusión de los pacientes en uno u otro grupo fue al azar.") }\end{array}$ \\
\hline $\begin{array}{l}\text { Allocation concealment } \\
\text { (selection bias) }\end{array}$ & Unclear risk & Not reported \\
\hline $\begin{array}{l}\text { Blinding of participants } \\
\text { and personnel (perfor- } \\
\text { mance bias) } \\
\text { All outcomes }\end{array}$ & Unclear risk & Not reported \\
\hline
\end{tabular}


Pozo 1996 (Continued)

$\begin{array}{lll}\text { Blinding of outcome as- } & \text { Unclear risk } & \text { Not reported } \\ \text { sessment (detection bias) } & \end{array}$

All outcomes

\begin{tabular}{lll}
\hline $\begin{array}{l}\text { Incomplete outcome data } \\
\text { (attrition bias) } \\
\text { All outcomes }\end{array}$ & Low risk & $\begin{array}{l}\text { Data on main results appeared to be complete. Converted cases ( } \mathrm{n}=2,10 \%) \\
\text { were apparently analysed by ITT. }\end{array}$ \\
\hline $\begin{array}{l}\text { Selective reporting (re- } \\
\text { porting bias) }\end{array}$ & Unclear risk & No distinction between primary and secondary outcome \\
\hline Other bias & Low risk & $\begin{array}{l}\text { Operations were done by surgical residents under supervision of either a se- } \\
\text { nior surgeon (in OA cases) or an experienced laparoscopic surgeon (in LA cas- } \\
\text { es). }\end{array}$ \\
\hline
\end{tabular}

\section{Reiertsen 1997}

\begin{tabular}{|c|c|}
\hline Methods & $\begin{array}{l}\text { Randomised controlled trial } \\
\text { Centres: one (Norway) }\end{array}$ \\
\hline Participants & $\begin{array}{l}\text { Participants: patients between } 18 \text { and } 60 \text { yr with suspected appendicitis. Pregnancy was an exclusion } \\
\text { criterion. } \\
\text { Gender: } 32 \% \text { females } \\
\text { Age: } 33.9 \text { and } 32.9 \text { years (mean) in LA and OA } \\
\text { Histology: Participants with un-inflamed appendices or other pathologies were excluded from the } \\
\text { analysis. }\end{array}$ \\
\hline Interventions & $\begin{array}{l}\text { LA: three trocars }(10,10 \text {, and } 5 \mathrm{~mm}) \text {; stump closure by Roeder loops }(n=42) \\
\text { OA: via muscle-splitting incision }(n=42) \\
\text { Antibiotics: metronidazole } 1 \mathrm{~g} \text { rectally }\end{array}$ \\
\hline Outcomes & Hospital stay; return to normal activities; complication rates; duration of operation \\
\hline Notes & $\begin{array}{l}\text { Follow-up: until return to normal activities } \\
\text { No appendectomy: was performed in } 12 \text { participants, of which } 5 \text { had other operations. All these cases } \\
\text { were not analysed according to ITT. } \\
\text { Declaration of interest: not reported } \\
\text { Funding: not reported } \\
\text { Preoperative imaging: not reported } \\
\text { Analgesia requirements: not reported } \\
\text { Sample size calculation: adequately reported }\end{array}$ \\
\hline
\end{tabular}

\section{Risk of bias}

\section{Bias}

Participants: patients between 18 and $60 \mathrm{yr}$ with suspected appendicitis. Pregnancy was an exclusion

Gender: 32\% females

Age: 33.9 and 32.9 years (mean) in LA and OA

Histology: Participants with un-inflamed appendices or other pathologies were excluded from the

LA: three trocars $(10,10$, and $5 \mathrm{~mm})$; stump closure by Roeder loops $(\mathrm{n}=42)$

OA: via muscle-splitting incision $(n=42)$

\begin{tabular}{|c|c|c|}
\hline $\begin{array}{l}\text { Random sequence genera- } \\
\text { tion (selection bias) }\end{array}$ & Unclear risk & $\begin{array}{l}\text { "The patients were equally allocated to laparoscopic or conventional appen- } \\
\text { dicectomy by block-randomization with a random block size between four and } \\
\text { 12." }\end{array}$ \\
\hline
\end{tabular}


Reiertsen 1997 (Continued)

\begin{tabular}{lll}
$\begin{array}{l}\text { Allocation concealment } \\
\text { (selection bias) }\end{array}$ & Unclear risk & Not reported \\
\hline $\begin{array}{l}\text { Blinding of participants } \\
\text { and personnel (perfor- } \\
\text { mance bias) }\end{array}$ & High risk & "The study was not carried out blindly" \\
All outcomes & &
\end{tabular}

\begin{tabular}{|c|c|c|}
\hline $\begin{array}{l}\text { Blinding of outcome as- } \\
\text { sessment (detection bias) }\end{array}$ & High risk & "The study was not carried out blindly" \\
\hline
\end{tabular}

\begin{tabular}{|c|c|c|}
\hline $\begin{array}{l}\text { Incomplete outcome data } \\
\text { (attrition bias) } \\
\text { All outcomes }\end{array}$ & High risk & $\begin{array}{l}\text { Of } 108 \text { randomised participants, } 24 \text { ( } 14 \text { in LA and } 10 \text { in OA group) were exclud- } \\
\text { ed from the analyses because they did not have appendectomy or histologi- } \\
\text { cally confirmed appendicitis ("included erroneously"). There were no conver- } \\
\text { sions. Completeness of follow-up was not explicitly described but appeared to } \\
\text { be } 100 \% \text {. }\end{array}$ \\
\hline $\begin{array}{l}\text { Selective reporting (re- } \\
\text { porting bias) }\end{array}$ & Unclear risk & No distinction between primary and secondary outcome \\
\hline Other bias & Low risk & $\begin{array}{l}\text { Surgeons were "experienced in both laparoscopic and conventional appen- } \\
\text { dectomy". }\end{array}$ \\
\hline
\end{tabular}

Ricca 2007

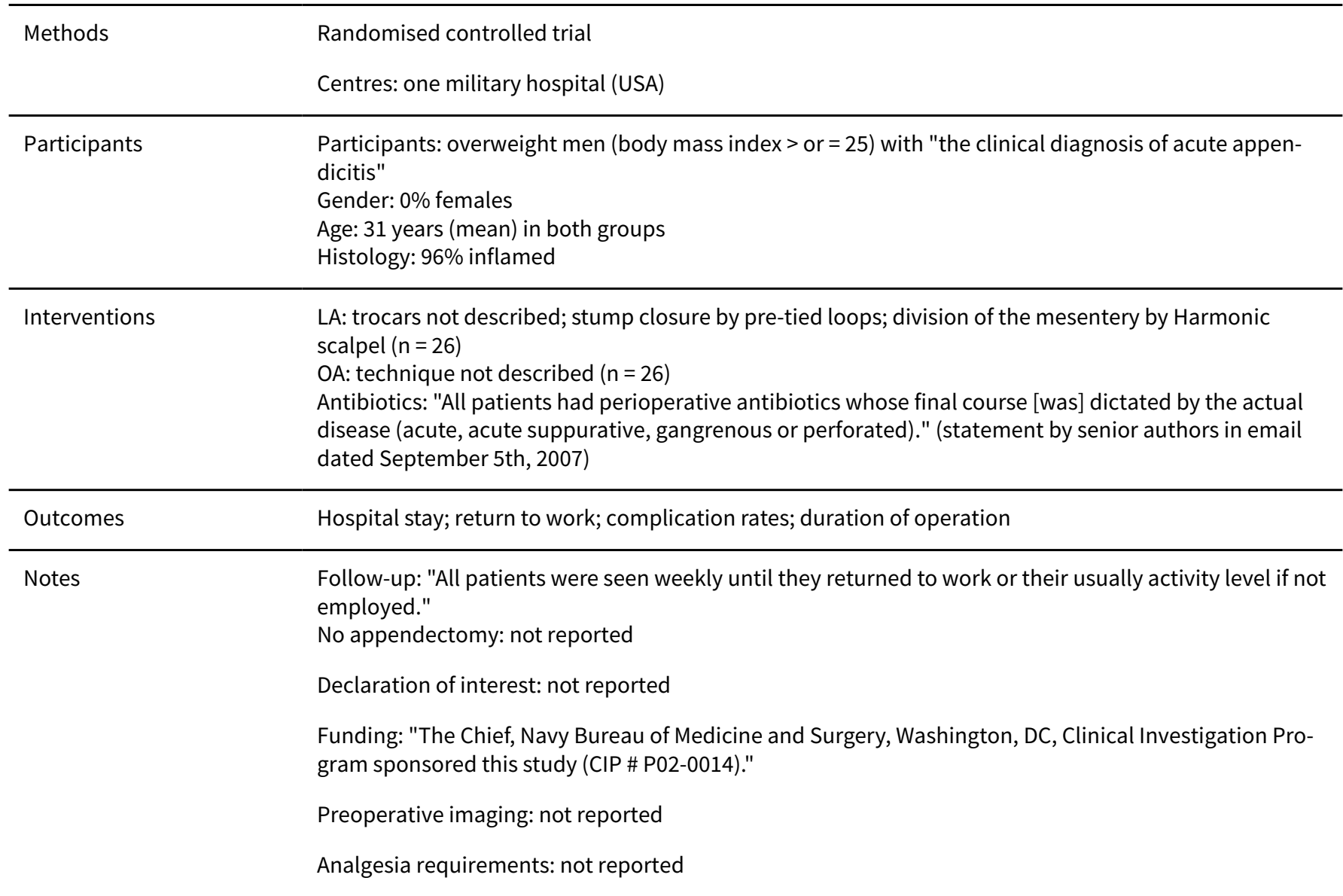


Ricca 2007 (Continued)

Sample size calculation: not reported

\section{Risk of bias}

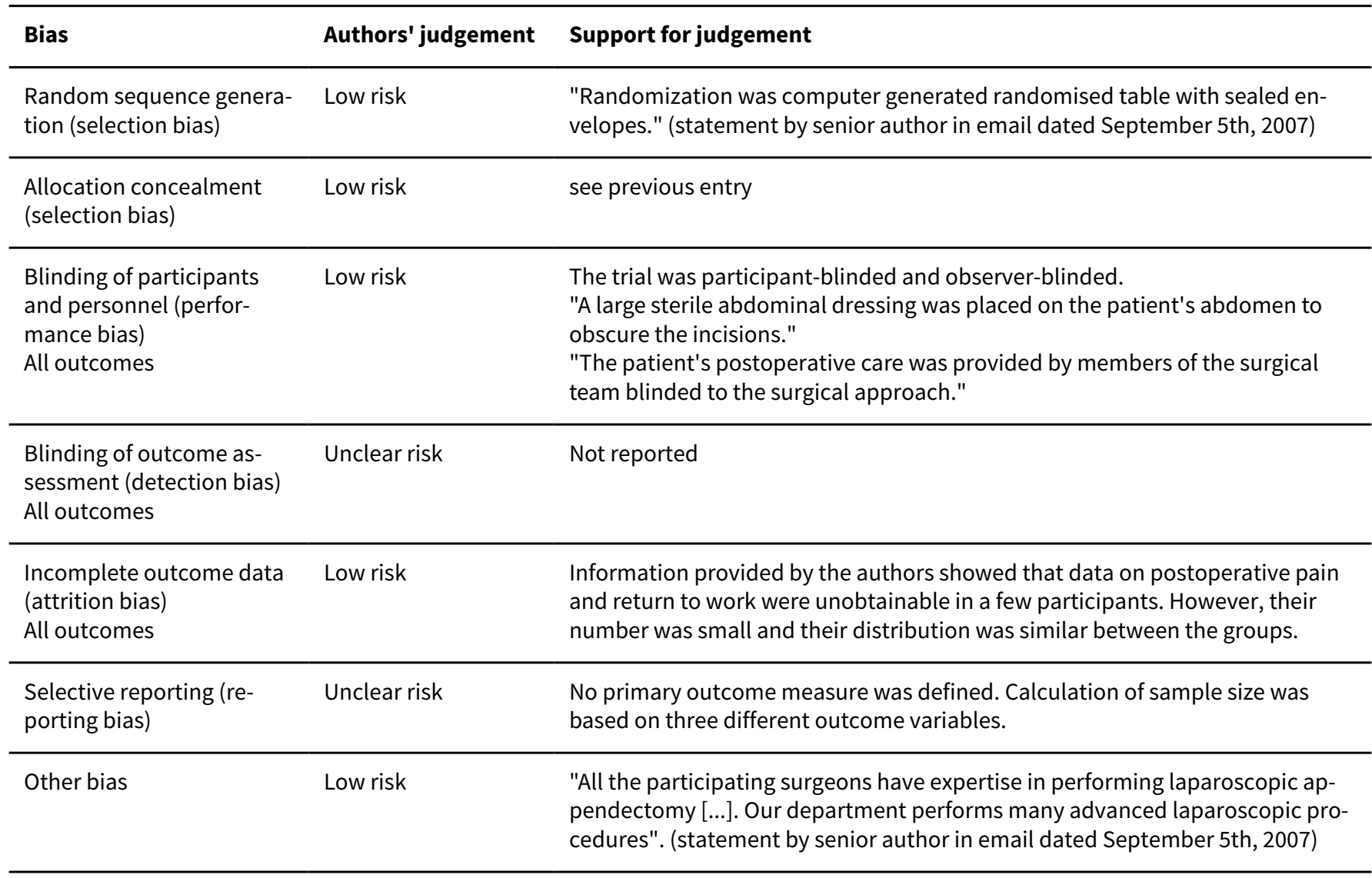

Saha 2010

\begin{tabular}{ll} 
Methods & Randomised controlled trial \\
& Centres: one (Bangladesh) \\
\hline Participants & $\begin{array}{l}\text { Participants: "children of < } 12 \text { years age with suspected acute appendicitis" } \\
\text { Gender: not reported } \\
\text { Age: } 8 \text { and } 7.2 \text { years (mean) in LA and OA } \\
\text { Histology: not reported }\end{array}$ \\
\hline Interventions & $\begin{array}{l}\text { LA: three trocar technique (presumably 10, 5, and } 5 \mathrm{~mm} \text { ); stump closure technique not described (n = } \\
\text { OA: via Lanz incision ( } \mathrm{n}=30 \text { ) } \\
\text { Antibiotics: not reported }\end{array}$ \\
& $\begin{array}{l}\text { Hospital stay; complication rates; pain at 6, 24, 48, 72, 96h and on day } 7 \text { (simple rating scale); analgesics } \\
\text { consumption; FLACC Scale }\end{array}$ \\
\hline Outcomes & $\begin{array}{l}\text { Follow-up: } 7 \text { days } \\
\text { No appendectomy: not reported } \\
\text { Declaration of interest: not reported }\end{array}$ \\
\hline Notes
\end{tabular}


Saha 2010 (Continued)

Funding: not reported

Preoperative imaging: not reported

Analgesia requirements: The consumption of pethidine, NSAIDs and paracetamol was lower in the laparoscopic group $(P=0.0001)$.

Sample size calculation: not reported

\section{Risk of bias}

\begin{tabular}{|c|c|c|}
\hline Bias & Authors' judgement & Support for judgement \\
\hline $\begin{array}{l}\text { Random sequence genera- } \\
\text { tion (selection bias) }\end{array}$ & Low risk & $\begin{array}{l}\text { "Grouping of patients were made by simple random technique by means [of a] } \\
\text { lottery with a box having equal amount of some slips written with laparoscop- } \\
\text { ic appendectomy (LA) and open appendectomy (OA)." }\end{array}$ \\
\hline $\begin{array}{l}\text { Allocation concealment } \\
\text { (selection bias) }\end{array}$ & Low risk & $\begin{array}{l}\text { "Parents [...] were requested to draw one of the slip[s] from [misspelled as } \\
\text { 'form'] the box blindly". }\end{array}$ \\
\hline $\begin{array}{l}\text { Blinding of participants } \\
\text { and personnel (perfor- } \\
\text { mance bias) } \\
\text { All outcomes }\end{array}$ & Unclear risk & Not reported \\
\hline $\begin{array}{l}\text { Blinding of outcome as- } \\
\text { sessment (detection bias) } \\
\text { All outcomes }\end{array}$ & Unclear risk & Not reported \\
\hline $\begin{array}{l}\text { Incomplete outcome data } \\
\text { (attrition bias) } \\
\text { All outcomes }\end{array}$ & Low risk & $\begin{array}{l}\text { Data on main results appeared to be complete. No conversions or dropouts } \\
\text { were mentioned. }\end{array}$ \\
\hline $\begin{array}{l}\text { Selective reporting (re- } \\
\text { porting bias) }\end{array}$ & Unclear risk & No primary outcome measure was defined. \\
\hline Other bias & Unclear risk & The surgeon's skills were not described. \\
\hline
\end{tabular}

Schietroma 2012

\begin{tabular}{ll}
\hline Methods & Randomised controlled trial \\
& Centres: one (Italy) \\
\hline Participants & Participants: "presenting with the clinical diagnosis of perforated appendicitis" \\
& Gender: $56 \%$ females \\
& Age: 40.6 and 42.4 years (mean) in LA and OA \\
& Histology: Histology results were not reported. Participants, in whom perforated appendicitis was not \\
& confirmed intraoperatively, were excluded from all analyses.
\end{tabular}

Interventions

LA: three trocar technique (sizes not mentioned); stump closure technique not described $(n=69)$

$O A$ : via McBurney or inferior midline incision $(n=73)$

Antibiotics: cefotaxime ( $2 \mathrm{~g}$ IV every $8 \mathrm{~h}$ ) and tobramycin (100 mg IV every $12 \mathrm{~h}$ ) initially and for 5 to 9

days after surgery

Outcomes Hospital stay; complication rates; duration of operation; immunological markers 
Schietroma 2012 (Continued)

Notes
Follow-up: "attempted to see all patients again 1 and 6 months after surgery"

No appendectomy: not reported

Declaration of interest: not reported

Funding: not reported

Preoperative imaging: not reported

Analgesia requirements: "All patients received... for pain relief, ketorolac trometamine (30 mg intravenous every 6 hours)."

Sample size calculation: not reported

\section{Risk of bias}

\begin{tabular}{|c|c|c|}
\hline Bias & Authors' judgement & Support for judgement \\
\hline $\begin{array}{l}\text { Random sequence genera- } \\
\text { tion (selection bias) }\end{array}$ & Low risk & $\begin{array}{l}\text { "Patients were randomly assigned [...] according to a computer-generated ta- } \\
\text { ble of random numbers." }\end{array}$ \\
\hline $\begin{array}{l}\text { Allocation concealment } \\
\text { (selection bias) }\end{array}$ & Unclear risk & "Randomization was performed by an independent computer consultant." \\
\hline $\begin{array}{l}\text { Blinding of participants } \\
\text { and personnel (perfor- } \\
\text { mance bias) } \\
\text { All outcomes }\end{array}$ & Unclear risk & Not reported \\
\hline $\begin{array}{l}\text { Blinding of outcome as- } \\
\text { sessment (detection bias) } \\
\text { All outcomes }\end{array}$ & Unclear risk & Not reported \\
\hline $\begin{array}{l}\text { Incomplete outcome data } \\
\text { (attrition bias) } \\
\text { All outcomes }\end{array}$ & High risk & $\begin{array}{l}\text { Participants with appendicitis but no visible perforation ( } n=15 ; 9 \%) \text { were ex- } \\
\text { cluded. In an email dated November } 11 \text { th, } 2012 \text {, the first author stated that } \\
\text { these } 15 \text { exclusions were distributed evenly across the two groups ( } 8 \text { in LA and } \\
7 \text { in OA group). } \\
\text { Conversions from LA to OA ( } n=4 ; 5 \%) \text { were excluded from the study, which vi- } \\
\text { olated the ITT principle. Additional data on these } 4 \text { converted cases were ob- } \\
\text { tained from the authors, thus allowing ITT analysis. } \\
\text { Losses to follow-up were not mentioned but appeared to be frequent, since } \\
\text { follow-up was only "attempted". }\end{array}$ \\
\hline $\begin{array}{l}\text { Selective reporting (re- } \\
\text { porting bias) }\end{array}$ & Unclear risk & No distinction between primary and secondary outcomes \\
\hline Other bias & Unclear risk & The surgeon's skills were not described. \\
\hline
\end{tabular}

\section{Schippers 1997}

\begin{tabular}{ll}
\hline Methods & Randomised controlled trial \\
& Centres: one (Germany) \\
\hline
\end{tabular}

\section{Participants}

Participants: adults and children $>4 \mathrm{yr}$ with a diagnosis of acute appendicitis Gender: 58\% females 
Schippers 1997 (Continued)

Age: 16 and $19 \mathrm{yr}$. (means)

Histology: $88 \%$ inflamed

\begin{tabular}{ll}
\hline Interventions & $\begin{array}{l}\text { LA: three trocar technique }(11,11, \text { and } 5.5 \mathrm{~mm}) ; \text { stump closure by ligatures }(\mathrm{n}=20) \\
\text { OA: via muscle-splitting incision }(\mathrm{n}=20) \\
\text { Antibiotics: not reported }\end{array}$ \\
\hline Outcomes & $\begin{array}{l}\text { Hospital stay; return to normal activities; complication rates; duration of operation; pain (VAS); lung } \\
\text { function; resumption of regular diet; mobilisation; cosmesis }\end{array}$ \\
\hline Notes & $\begin{array}{l}\text { Follow-up: } 6 \text { months after the operation } \\
\text { No appendectomy: none }\end{array}$ \\
& Declaration of interest: not reported \\
& Funding: not reported \\
& Preoperative imaging: not reported \\
& Analgesia requirements: not reported \\
& Sample size calculation: not reported
\end{tabular}

\section{Risk of bias}

\begin{tabular}{lll}
\hline Bias & Authors' judgement & Support for judgement \\
\hline $\begin{array}{l}\text { Random sequence genera- } \\
\text { tion (selection bias) }\end{array}$ & Unclear risk & "Patients were randomised..." \\
\hline $\begin{array}{l}\text { Allocation concealment } \\
\text { (selection bias) }\end{array}$ & Low risk & "...by drawing envelopes." \\
\hline $\begin{array}{l}\text { Blinding of participants } \\
\text { and personnel (perfor- } \\
\text { mance bias) }\end{array}$ & Unclear risk & Not reported \\
All outcomes & \\
\hline
\end{tabular}

\begin{tabular}{lll}
$\begin{array}{l}\text { Blinding of outcome as- } \\
\text { sessment (detection bias) } \\
\text { All outcomes }\end{array}$ & Unclear risk & Not reported \\
\hline $\begin{array}{l}\text { Incomplete outcome data } \\
\text { (attrition bias) } \\
\text { All outcomes }\end{array}$ & Low risk & No conversions or losses to follow-up mentioned \\
\hline $\begin{array}{l}\text { Selective reporting (re- } \\
\text { porting bias) }\end{array}$ & Unclear risk & No distinction between primary and secondary outcome \\
\hline Other bias & Low risk & Surgeons were described as "experienced". \\
\hline
\end{tabular}

\section{Settmacher 1995}

\begin{tabular}{ll}
\hline Methods & Randomised controlled trial \\
& Centres: one (Germany) \\
\hline Participants & Participants: adults with typical clinical symptoms \\
\hline
\end{tabular}


Settmacher 1995 (Continued)

Gender: $56 \%$ females

Age: 32.2 and 23.6 years (mean) in LA and OA

Histology: not given, but all participants had leukocyte counts above 8000 per microlitre

\begin{tabular}{|c|c|}
\hline Interventions & $\begin{array}{l}\text { LA: technique not described }(n=12) \\
\text { OA: technique not described }(n=11) \\
\text { Antibiotics: not mentioned }\end{array}$ \\
\hline Outcomes & Duration of surgery; immunological markers \\
\hline \multirow[t]{6}{*}{ Notes } & $\begin{array}{l}\text { Follow-up: not reported } \\
\text { No appendectomy: none described }\end{array}$ \\
\hline & Declaration of interest: not reported \\
\hline & Funding: not reported \\
\hline & Preoperative imaging: not reported \\
\hline & Analgesia requirements: not reported \\
\hline & Sample size calculation: not reported \\
\hline
\end{tabular}

\section{Risk of bias}

\begin{tabular}{lll}
\hline Bias & Authors' judgement & Support for judgement \\
\hline $\begin{array}{l}\text { Random sequence genera- } \\
\text { tion (selection bias) }\end{array}$ & Unclear risk & "In this prospective randomized study..." \\
\hline $\begin{array}{l}\text { Allocation concealment } \\
\text { (selection bias) }\end{array}$ & Low risk & $\begin{array}{l}\text { On personal contact, the primary author stated that the trial had been ran- } \\
\text { domised by drawing envelopes. }\end{array}$ \\
\hline $\begin{array}{l}\text { Blinding of participants } \\
\text { and personnel (perfor- } \\
\text { mance bias) }\end{array}$ & Unclear risk & Not reported \\
All outcomes & & \\
\hline
\end{tabular}

\begin{tabular}{lll}
$\begin{array}{l}\text { Blinding of outcome as- } \\
\text { sessment (detection bias) } \\
\text { All outcomes }\end{array}$ & Unclear risk & Not reported \\
\hline $\begin{array}{l}\text { Incomplete outcome data } \\
\begin{array}{l}\text { (attrition bias) } \\
\text { All outcomes }\end{array}\end{array}$ & Unclear risk & $\begin{array}{l}\text { No reporting of the number and reasons for exclusions or completeness of fol- } \\
\text { low-up. Conversions not mentioned }\end{array}$ \\
\hline
\end{tabular}

All outcomes

Selective reporting (re- Low risk $\quad$ Two outcomes were adequately described.
porting bias)

porting bias)

Other bias Unclear risk The surgeon's skills were not described.

\section{Sezeur 1997}

\begin{tabular}{ll}
\hline Methods & Randomised controlled trial \\
& Centres: one (France) \\
\hline Participants & Participants: adults $>16 \mathrm{yr}$ with a diagnosis of acute appendicitis requiring appendectomy.
\end{tabular}

Laparoscopic versus open surgery for suspected appendicitis (Review) 
Sezeur 1997 (Continued)

Gender: 31\% females

Age: 27 and 28 years (median) in LA and OA

Histology: 94\% inflamed

\begin{tabular}{ll}
\hline Interventions & LA: not described $(n=15)$ \\
& OA: via McBurney incision $(n=17)$ \\
& Antibiotics: 2 g amoxicillin and $200 \mathrm{mg}$ augmentin ${ }^{\circledast}$ IV
\end{tabular}

\begin{tabular}{ll}
\hline Outcomes & Complication rates; duration of operation; rates of infected blood cultures \\
\hline Notes & Follow-up: not reported \\
No appendectomy: All participants underwent appendectomy. \\
Declaration of interest: not reported \\
Funding: not reported \\
Preoperative imaging: not reported \\
Analgesia requirements: not reported \\
Sample size calculation: not reported
\end{tabular}

\section{Risk of bias}

\begin{tabular}{lll}
\hline Bias & Authors' judgement & Support for judgement \\
\hline $\begin{array}{l}\text { Random sequence genera- } \\
\text { tion (selection bias) }\end{array}$ & Unclear risk & Not reported \\
\hline $\begin{array}{l}\text { Allocation concealment } \\
\text { (selection bias) }\end{array}$ & Low risk & In a letter, the authors stated that sealed envelopes were used. \\
\hline $\begin{array}{l}\text { Blinding of participants } \\
\text { and personnel (perfor- } \\
\text { mance bias) } \\
\text { All outcomes }\end{array}$ & Unclear risk & Not reported \\
\hline
\end{tabular}

\begin{tabular}{lll}
$\begin{array}{l}\text { Blinding of outcome as- } \\
\text { sessment (detection bias) } \\
\text { All outcomes }\end{array}$ & Unclear risk & Not reported \\
\hline $\begin{array}{l}\text { Incomplete outcome data } \\
\text { (attrition bias) }\end{array}$ & High risk & $\begin{array}{l}\text { Conversions }(n=3 ; 17 \%) \text { were excluded from the study, which violated the ITT } \\
\text { principle. Losses to follow-up were not mentioned but appeared to be negligi- } \\
\text { ble. }\end{array}$
\end{tabular}

\begin{tabular}{lll}
\hline $\begin{array}{l}\text { Selective reporting (re- } \\
\text { porting bias) }\end{array}$ & Unclear risk & Primary outcome (rates of infected blood cultures) was described. \\
\hline Other bias & Unclear risk & The surgeon's skills were not described. \\
\hline
\end{tabular}

\section{Simon 2009}

\begin{tabular}{ll}
\hline Methods & Randomised controlled trial \\
& Centres: not reported (Germany) \\
\hline Participants & Participants: children who presented with the clinical diagnosis of appendicitis \\
\hline
\end{tabular}


Simon 2009 (Continued)

Gender: not reported

Age: 11.5 and 10.7 years (mean) in LA and OA

Histology: not reported

Interventions
OA: 3 -trocar technique $(12,5$, and $5 \mathrm{~mm})$, stump dissection by Endo-GIA $(n=20)$

$O A$ : muscle-sparing right lower quadrant incision $(n=20)$

Antibitotics: not mentioned

\begin{tabular}{|c|c|}
\hline Outcomes & Body temperature, leukocyte count, tumour necrosis factor, length of hospital stay \\
\hline \multirow[t]{6}{*}{ Notes } & $\begin{array}{l}\text { Follow-up: not reported } \\
\text { No appendectomy: not reported }\end{array}$ \\
\hline & Declaration of interest: not reported \\
\hline & Funding: not reported \\
\hline & Preoperative imaging: not reported \\
\hline & Analgesia requirements: not reported \\
\hline & Sample size calculation: not reported \\
\hline
\end{tabular}

\section{Risk of bias}

\begin{tabular}{lll}
\hline Bias & Authors' judgement & Support for judgement \\
\hline $\begin{array}{l}\text { Random sequence genera- } \\
\text { tion (selection bias) }\end{array}$ & Low risk & "[The children] were randomized..." \\
\hline $\begin{array}{l}\text { Allocation concealment } \\
\text { (selection bias) }\end{array}$ & Unclear risk & "... by pulling lots..." \\
\hline $\begin{array}{l}\text { Blinding of participants } \\
\text { and personnel (perfor- } \\
\text { mance bias) }\end{array}$ & Unclear risk & Not reported \\
All outcomes & \\
\hline
\end{tabular}

\begin{tabular}{lll}
$\begin{array}{l}\text { Blinding of outcome as- } \\
\text { sessment (detection bias) } \\
\text { All outcomes }\end{array}$ & Unclear risk & Not reported \\
\hline $\begin{array}{l}\text { Incomplete outcome data } \\
\text { (attrition bias) }\end{array}$ & High risk & $\begin{array}{l}\text { No conversions mentioned. "Retrospectively, four patients in the open group } \\
\text { all outcomes }\end{array}$ \\
$\begin{array}{l}\text { perforated appendicitis was described on their histopathological report, leav- } \\
\text { ing an equal number of patients in each study cohort }(n=20 \text { each)." Therefore, } \\
\text { the ITT principle was violated. } \\
\text { No reporting of number and reasons for exclusions or completeness of fol- } \\
\text { low-up }\end{array}$
\end{tabular}

\begin{tabular}{lll}
\hline $\begin{array}{l}\text { Selective reporting (re- } \\
\text { porting bias) }\end{array}$ & Unclear risk & No distinction between primary and secondary outcome \\
\hline Other bias & Unclear risk & The surgeon's skills were not described. \\
\hline
\end{tabular}

Singh 2017

Methods Randomised controlled trial


Singh 2017 (Continued)

Centres: one (India)

Comment: This trial had three arms. Data on participants $(n=15)$ treated by single incision laparoscopic appendectomy were not extracted.

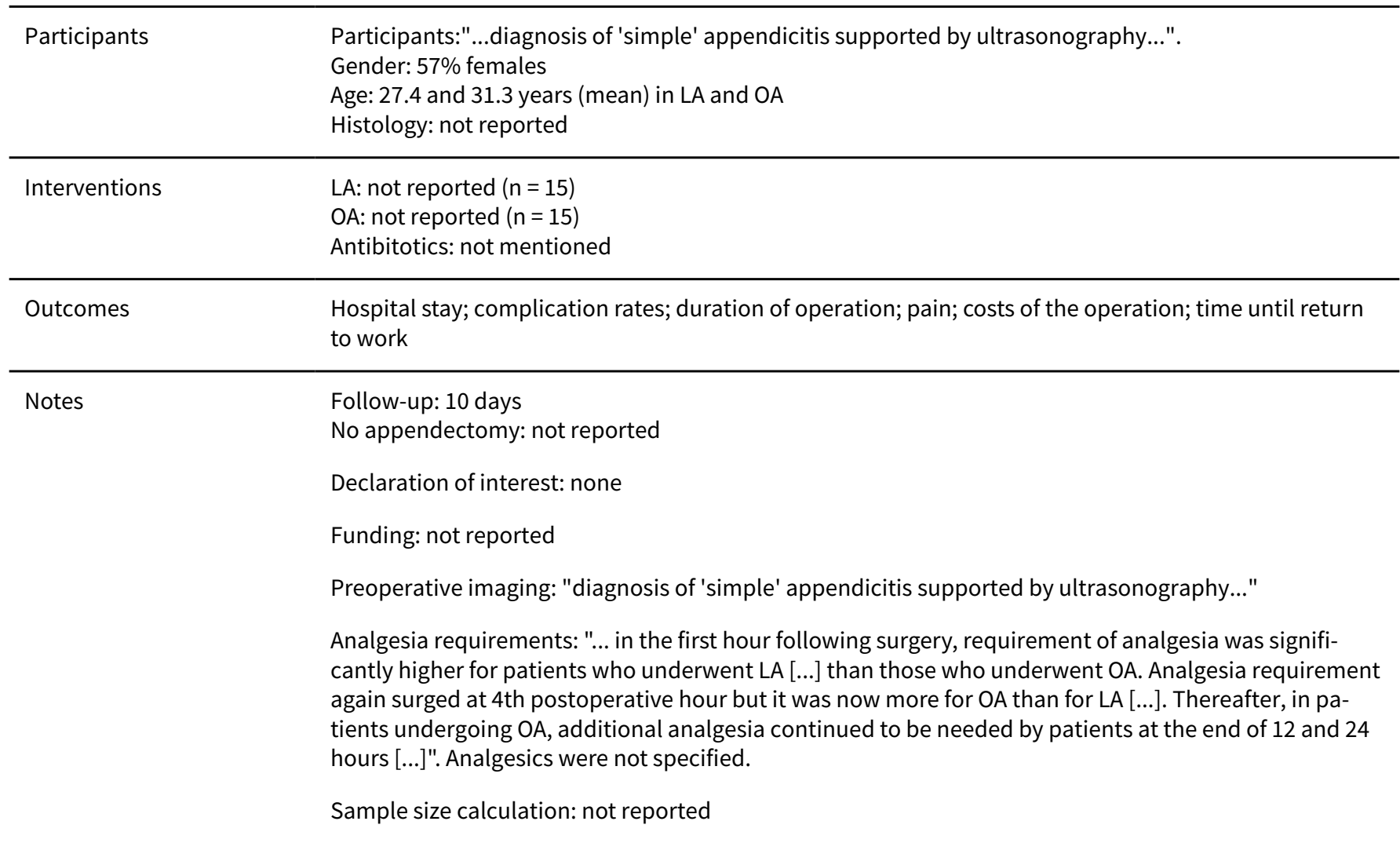

\section{Risk of bias}

\begin{tabular}{|c|c|c|}
\hline Bias & Authors' judgement & Support for judgement \\
\hline $\begin{array}{l}\text { Random sequence genera- } \\
\text { tion (selection bias) }\end{array}$ & Low risk & "...randomized using computer generated random numbers..." \\
\hline $\begin{array}{l}\text { Allocation concealment } \\
\text { (selection bias) }\end{array}$ & Unclear risk & Not reported \\
\hline $\begin{array}{l}\text { Blinding of participants } \\
\text { and personnel (perfor- } \\
\text { mance bias) } \\
\text { All outcomes }\end{array}$ & Low risk & $\begin{array}{l}\text { "All patients were operated under general anaesthesia and abdomen was cov- } \\
\text { ered with a large dressing which was removed only after } 24 \text { hours to ensure } \\
\text { blinding at patients end." }\end{array}$ \\
\hline $\begin{array}{l}\text { Blinding of outcome as- } \\
\text { sessment (detection bias) } \\
\text { All outcomes }\end{array}$ & Low risk & "Blinding was also ensured [...] for recording of data and last analysis." \\
\hline $\begin{array}{l}\text { Incomplete outcome data } \\
\text { (attrition bias) } \\
\text { All outcomes }\end{array}$ & Low risk & $\begin{array}{l}\text { No losses from follow-up visible. No conversions or other changes in surgical } \\
\text { procedure }\end{array}$ \\
\hline $\begin{array}{l}\text { Selective reporting (re- } \\
\text { porting bias) }\end{array}$ & Unclear risk & No distinction between primary and secondary outcome \\
\hline
\end{tabular}


Singh 2017 (Continued)
Other bias
Unclear risk
The surgeon's skills were not described.

Sozutek 2013

Randomised controlled trial
Centres: one (Turkey)
Comment: This trial had three arms. Data on participants $(\mathrm{n}=25)$ treated by single incision laparoscop-
ic appendectomy were not extracted.

\begin{tabular}{|c|c|}
\hline Participants & $\begin{array}{l}\text { Participants: "older than } 18 \text { years presenting with acute appendicitis" } \\
\text { Gender: } 58 \% \text { females } \\
\text { Age: } 30 \text { and } 32.2 \text { years (mean) in LA and OA } \\
\text { Histology: } 100 \% \text { inflamed }\end{array}$ \\
\hline Interventions & $\begin{array}{l}\text { LA: three trocar technique }(10,5 \text {, and } 5 \mathrm{~mm}) \text {; stump closure technique not described }(n=25) \\
\text { OA: via McBurney incision }(n=25) \\
\text { Antibiotics: } 1 \text { g cefazolin sodium, metronidazole } 0.5 \% 100 \mathrm{~mL} \text { if perforation or abscess detected intra- } \\
\text { operatively }\end{array}$ \\
\hline Outcomes & $\begin{array}{l}\text { Pain severity (VAS Score, primary outcome) at } 3,6,12 \text {, and } 24 \mathrm{~h} \text {, length of hospital stay, time of surgery, } \\
\text { wound infection, intra-abdominal abscess, cosmesis (first month after surgery using mini survey creat- } \\
\text { ed by Kapischke 2011) }\end{array}$ \\
\hline
\end{tabular}

Follow-up: 1 week and 1 month after surgery
No appendectomy: none described
Declaration of interest: "The authors declare no conflict of interest"
Funding: not reported
Preoperative imaging: "The findings were verified with ultrasonography or computed tomography in
case of suspected appendicitis."
Analgesia requirements: "... analgesic requirement of the patients in OA group were significantly high-
er..." Analgesics were not specified.
Sample size calculation: adequately reported

\section{Risk of bias}

\begin{tabular}{lll}
\hline Bias & Authors' judgement & Support for judgement \\
\hline $\begin{array}{l}\text { Random sequence genera- } \\
\text { tion (selection bias) }\end{array}$ & Low risk & "according to the computer-generated randomizing list..." \\
\hline $\begin{array}{l}\text { Allocation concealment } \\
\text { (selection bias) }\end{array}$ & Low risk & "... that was used by independent computer consultant " \\
& & $\begin{array}{l}\text { In an email dated December } 5 \text { th, 2014, the first author added that the comput- } \\
\text { er consultant: "was out of the study" }\end{array}$ \\
\hline $\begin{array}{l}\text { Blinding of participants } \\
\text { and personnel (perfor- } \\
\text { mance bias) }\end{array}$ & Unclear risk & Not reported \\
$\begin{array}{l}\text { All outcomes } \\
\end{array}$ & \\
\hline
\end{tabular}


Sozutek 2013 (Continued)
Blinding of outcome as-
Unclear risk
Not reported sessment (detection bias)
All outcomes

\begin{tabular}{lll}
\hline $\begin{array}{l}\text { Incomplete outcome data } \\
\text { (attrition bias) } \\
\text { All outcomes }\end{array}$ & Low risk & $\begin{array}{l}\text { There were no conversions. Analyses were apparently done following the ITT } \\
\text { principle. Losses to follow-up were not described in detail. }\end{array}$ \\
\hline $\begin{array}{l}\text { Selective reporting (re- } \\
\text { porting bias) }\end{array}$ & Low risk & $\begin{array}{l}\text { The primary outcome (postoperative pain) was adequately described. The } \\
\text { study was therefore judged to have low risk of bias, although neither a study } \\
\text { protocol nor a study registration were available. }\end{array}$
\end{tabular}

\begin{tabular}{ll}
\hline Other bias & Low risk \\
& $\begin{array}{l}\text { "All operations were performed... by same } 2 \text { specialist surgeons ... and their } \\
\text { residents". The study authors noted a learning curve in the third arm of the } \\
\text { study (single incision laparoscopic appendectomy), but this was irrelevant } \\
\text { when comparing OA and standard LA. }\end{array}$ \\
\hline
\end{tabular}

\section{Stare 1998}

\begin{tabular}{|c|c|}
\hline Methods & $\begin{array}{l}\text { Randomised controlled trial } \\
\text { Centres: one (Croatia) }\end{array}$ \\
\hline Participants & $\begin{array}{l}\text { Participants: adults }>16 \text { yr "with a clinical diagnosis of acute appendicitis" } \\
\text { Gender: } 53 \% \text { females. } \\
\text { Age: } 35 \text { and } 40 \text { years (mean) in LA and OA } \\
\text { Histology: } 88 \% \text { inflamed }\end{array}$ \\
\hline Interventions & $\begin{array}{l}\text { LA: three trocar technique; stump closure with Vicryl endo loops }(n=48) \\
\text { OA: via McBurney muscle-splitting incision }(n=26) \\
\text { Antibiotics: were given to all participants. Drug not stated }\end{array}$ \\
\hline Outcomes & Hospital stay; return to normal activities; complication rates; duration of operation \\
\hline \multirow[t]{6}{*}{ Notes } & $\begin{array}{l}\text { Follow-up: "Patients were questioned by telephone and by a mailed questionnaire." } \\
\text { No appendectomy: none. "A normal appendix was always removed." }\end{array}$ \\
\hline & Declaration of interest: not reported \\
\hline & Funding: not reported \\
\hline & Preoperative imaging: not reported \\
\hline & Analgesia requirements: not reported \\
\hline & Sample size calculation: not reported \\
\hline
\end{tabular}

\section{Risk of bias}

\begin{tabular}{lll}
\hline Bias & Authors' judgement & Support for judgement \\
\hline $\begin{array}{l}\text { Random sequence genera- } \\
\text { tion (selection bias) }\end{array}$ & Unclear risk & "Patients were randomised..." \\
\hline $\begin{array}{l}\text { Allocation concealment } \\
\text { (selection bias) }\end{array}$ & Low risk & $\begin{array}{l}\text { On personal contact, the authors stated that sealed envelopes were used. } \\
\text { They had no explanation for the uneven group sizes in the trial. }\end{array}$ \\
\hline
\end{tabular}




\section{Stare 1998 (Continued)}

Blinding of participants and personnel (performance bias)

All outcomes
High risk On personal contact, the authors stated that the trial was unblinded.

\section{Blinding of outcome as- High risk \\ On personal contact, the authors stated that the trial was unblinded. \\ sessment (detection bias)}

All outcomes

Incomplete outcome data Low risk (attrition bias)

All outcomes

Alloutcomes

No conversion were reported. Follow-up data appeared to be similarly complete in both groups.

Selective reporting (re- Unclear risk No distinction between primary and secondary outcome porting bias)

Other bias

Low risk

All operations "were performed or supervised by a senior surgeon."

\section{Sun 1998}

\begin{tabular}{|c|c|}
\hline Methods & $\begin{array}{l}\text { Randomised controlled trial } \\
\text { Centres: one (China) } \\
\text { Comment: The study had a three-armed design. In the third group ( } \mathrm{n}=100 \text { participants), a small inci- } \\
\text { sion was made in the right lower abdomen. "With the help of laparoscopy, the appendix was identified } \\
\text { and moved outside the abdomen, and then resected." In the present review, the review authors exclud- } \\
\text { ed the results from this group, as this procedure is neither LA nor diagnostic laparoscopy. }\end{array}$ \\
\hline Participants & $\begin{array}{l}\text { Participants: "with acute appendicitis" } \\
\text { Gender: } 45 \% \text { females } \\
\text { Age: } 34.4 \text { and } 38.7 \text { years (mean) in LA and OA } \\
\text { Histology: apparently distributed evenly }\end{array}$ \\
\hline Interventions & $\begin{array}{l}\text { LA: unclearly described, but performed under general anaesthesia }(n=50) \\
\text { OA: via a } 6-10 \mathrm{~cm} \text { "traditional" incision, under local anaesthesia }(n=100) \\
\text { Antibiotics: not reported }\end{array}$ \\
\hline Outcomes & $\begin{array}{l}\text { Hospital stay; complication rates; duration of operation; need for pain medication; return to normal ac- } \\
\text { tivities; hospital costs }\end{array}$ \\
\hline \multirow[t]{6}{*}{ Notes } & $\begin{array}{l}\text { Follow-up: not reported } \\
\text { No appendectomy: none }\end{array}$ \\
\hline & Declaration of interest: not reported \\
\hline & Funding: not reported \\
\hline & Preoperative imaging: not reported \\
\hline & Analgesia requirements: not reported \\
\hline & Sample size calculation: not reported \\
\hline
\end{tabular}

\section{Risk of bias}

Bias Authors' judgement Support for judgement


Sun 1998 (Continued)

Random sequence genera- Unclear risk Not mentioned. Translated quotation: "250 patients with acute appendicitis tion (selection bias) were randomly divided into 3 groups." The authors did not explain why the three groups were different in size (OA 100, LA 50, third technique 100 cases). Baseline characteristics were similar between the groups.

\begin{tabular}{lll}
\hline $\begin{array}{l}\text { Allocation concealment } \\
\text { (selection bias) }\end{array}$ & Unclear risk & Not reported \\
\hline $\begin{array}{l}\text { Blinding of participants } \\
\text { and personnel (perfor- } \\
\text { mance bias) }\end{array}$ & Unclear risk & Not reported \\
All outcomes & \\
\hline
\end{tabular}

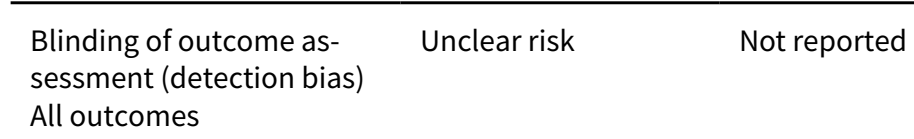

\begin{tabular}{|c|c|c|}
\hline $\begin{array}{l}\text { Incomplete outcome data } \\
\text { (attrition bias) }\end{array}$ & Low risk & $\begin{array}{l}\text { No conversions were reported. Follow-up data appeared to be similarly com- } \\
\text { plete in both groups. }\end{array}$ \\
\hline
\end{tabular}

All outcomes

No conversions were reported. Follow-up data appeared to be similarly com-

Selective reporting (re- Unclear risk No distinction between primary and secondary outcome
porting bias)

\begin{tabular}{|c|c|c|}
\hline Other bias & Unclear risk & The surgeon's skills were not described. \\
\hline
\end{tabular}

\section{Taguchi 2015}

\begin{tabular}{|c|c|}
\hline Methods & $\begin{array}{l}\text { Randomised controlled trial } \\
\text { Centres: one (Japan) }\end{array}$ \\
\hline Participants & $\begin{array}{l}\text { Participants: "diagnosed as having complicated appendicitis with peritonitis or abscess formation by } \\
\text { abdominal examination, laboratory data, or CT" } \\
\text { Gender: } 35 \% \text { females } \\
\text { Age: } 46 \text { and } 49 \text { years (median) in LA and OA } \\
\text { Histology: complicated appendicitis (perforation or an intra-abdominal abscess) (93\%), uncomplicat- } \\
\text { ed appendicitis (7\%) }\end{array}$ \\
\hline Interventions & $\begin{array}{l}\text { LA: two-handed, four-trocar technique }(12,5,5 \text {, and } 5 \mathrm{~mm}) \text {; none }(\mathrm{n}=1) \text {, ligation }(\mathrm{n}=2) \text { or a stapling ( } \mathrm{n} \\
=39 \text { ) device for appendiceal stump closure }(\mathrm{n}=42) \\
\text { OA: performed through a midline or pararectal incision; none }(\mathrm{n}=0) \text {, ligation }(\mathrm{n}=37) \text { or a stapling }(\mathrm{n}= \\
\text { 2) device for appendiceal stump closure }(n=39) \\
\text { Antibiotics: preoperative intravenous antibiotics (cefozopran hydrochloride } 1 \text { g every } 12 \mathrm{~h} \text { ), which } \\
\text { were continued in the postoperative period until the inflammatory response abated as comprehensive- } \\
\text { ly (clinical and laboratory findings) }\end{array}$ \\
\hline
\end{tabular}
Outcomes
Wound infection; operating time; analgesic use frequency; start of oral intake; recovery of bowel move- ment; restoration of physical activity; length of postoperative hospital stay; changes in the white blood cell count and CRP level after surgery

Follow-up: 30 days after surgery
No appendectomy: none
Declaration of interest: the authors "have no conflict of interest"
Funding: Nagoya Daini Red Cross Hospital (self-funding)


Taguchi 2015 (Continued)

Preoperative imaging: not reported

Analgesia requirements: "Analgesics were given intramuscularly (pentazosine), as a suppository (diclofenac sodium), or orally (loxoprofen) as needed." "... times of analgesic use did not differ significantly."

Sample size calculation: adequately reported

\section{Risk of bias}

\begin{tabular}{|c|c|c|}
\hline Bias & Authors' judgement & Support for judgement \\
\hline $\begin{array}{l}\text { Random sequence genera- } \\
\text { tion (selection bias) }\end{array}$ & Low risk & $\begin{array}{l}\text { "Randomization was performed using a computer-based randomization pro- } \\
\text { gram (FileMaker Pro), ..." }\end{array}$ \\
\hline $\begin{array}{l}\text { Allocation concealment } \\
\text { (selection bias) }\end{array}$ & Low risk & $\begin{array}{l}\text { "... which allowed complete concealment of the randomization } \\
\text { sequence." }\end{array}$ \\
\hline $\begin{array}{l}\text { Blinding of participants } \\
\text { and personnel (perfor- } \\
\text { mance bias) } \\
\text { All outcomes }\end{array}$ & High risk & "Patients and investigators were not masked to group assignment" \\
\hline $\begin{array}{l}\text { Blinding of outcome as- } \\
\text { sessment (detection bias) } \\
\text { All outcomes }\end{array}$ & High risk & "Patients and investigators were not masked to group assignment" \\
\hline $\begin{array}{l}\text { Incomplete outcome data } \\
\text { (attrition bias) } \\
\text { All outcomes }\end{array}$ & Low risk & $\begin{array}{l}\text { One conversion was analysed according to the ITT principle. } \\
\text { " No patient was excluded after randomization, and no one was lost to fol- } \\
\text { low-up." }\end{array}$ \\
\hline $\begin{array}{l}\text { Selective reporting (re- } \\
\text { porting bias) }\end{array}$ & Unclear risk & Insufficient information to permit judgement \\
\hline Other bias & Low risk & $\begin{array}{l}\text { "All operations were performed by six senior surgeons with sufficient experi- } \\
\text { ence in laparoscopic surgery." }\end{array}$ \\
\hline
\end{tabular}

Tate 1993a

\begin{tabular}{ll}
\hline Methods & Randomised controlled trial \\
& Centres: one (China) \\
\hline Participants & $\begin{array}{l}\text { Participants: with a clinical diagnosis of appendicitis, suitable for laparoscopy } \\
\text { Gender: } 39 \% \text { females } \\
\text { Age: } 31.4 \text { and } 33 \text { years (mean) in LA and OA } \\
\text { Histology: } 86 \% \text { inflamed }\end{array}$ \\
\hline LAP: "a normal appendix was left when another definite cause of the patient's symptoms was found". \\
$\begin{array}{l}\text { LA: three trocar technique; stump closure with loop ligatures ( }=70 \text { ) } \\
\text { Antibiotics: } 1 \text { g cefotaxime and } 500 \text { mg metronidazole IV. }\end{array}$ \\
\hline Outcomes & $\begin{array}{l}\text { Hospital stay; return to normal activities; complication rates; duration of anaesthesia; return to liquid } \\
\text { and solid diet; pain (VAS); pain medication; nausea (VAS) }\end{array}$
\end{tabular}


Tate 1993a (Continued)

Notes
Follow-up: outpatient visit 2-4 weeks postoperatively

No appendectomy: was performed in $5(7 \%)$ laparoscopy participants, in whom another diagnosis was

made. They were analysed ITT.

Declaration of interest: not reported

Funding: not reported

Preoperative imaging: not reported

Analgesia requirements: "... $1 \mathrm{mg} / \mathrm{kg}$ pethidine intramuscularly every $3 \mathrm{~h}$ on demand or $1 \mathrm{~g}$ paracetamol by mouth every $4 \mathrm{~h}$ on demand." There was no difference in the consumption of analgesia.

Sample size calculation: not reported

\section{Risk of bias}

\begin{tabular}{lll} 
Bias & Authors' judgement & Support for judgement \\
\hline $\begin{array}{l}\text { Random sequence genera- } \\
\text { tion (selection bias) }\end{array}$ & Low risk & $\begin{array}{l}\text { "Computer-generated blocked random numbers were used to assign the type } \\
\text { of surgery,..." }\end{array}$ \\
\hline $\begin{array}{l}\text { Allocation concealment } \\
\text { (selection bias) }\end{array}$ & Low risk & "...which was written on a card sealed in a completely opaque envelope." \\
\hline $\begin{array}{l}\text { Blinding of participants } \\
\begin{array}{l}\text { and personnel (perfor- } \\
\text { mance bias) }\end{array}\end{array}$ & Unclear risk & Not reported \\
All outcomes & \\
\hline
\end{tabular}

\begin{tabular}{lll}
\hline $\begin{array}{l}\text { Blinding of outcome as- } \\
\text { sessment (detection bias) } \\
\text { All outcomes }\end{array}$ & Unclear risk & Not reported \\
\hline $\begin{array}{l}\text { Incomplete outcome data } \\
\text { (attrition bias) } \\
\text { All outcomes }\end{array}$ & Low risk & $\begin{array}{l}\text { Converted cases }(\mathrm{n}=14 ; 20 \%) \text { and those receiving other operations were } \\
\text { analysed according to ITT. Follow-up completeness was similar between the } \\
\text { groups (46 LA and 42 OA participants). }\end{array}$ \\
\hline $\begin{array}{l}\text { Selective reporting (re- } \\
\text { porting bias) }\end{array}$ & Unclear risk & No distinction between primary and secondary outcome \\
\hline $\begin{array}{l}\text { Other bias } \\
\text { High risk }\end{array}$ & $\begin{array}{l}\text { Surgeons: "All surgeons had completed formal laparoscopic training, but ... up } \\
\text { to } 50 \% \text { of conversions were probably due to effects of experience". }\end{array}$ \\
\hline
\end{tabular}

Thomson 2015

\begin{tabular}{ll}
\hline Methods & Randomised controlled trial \\
& Centres: one (South Africa) \\
\hline Participants & Participants: with "complicated appendicitis following clinical examination, biochemical tests and ra- \\
diological investigations" & Gender: $44 \%$ females \\
Age: 26.4 and 26.6 years (mean) in LA and OA \\
Histology: $100 \%$ inflamed
\end{tabular}

Interventions

LA: three trocar technique $(10,10$, and $5 \mathrm{~mm})$; stump closure technique not described $(n=60)$

$O A$ : via McBurney incision or via lower midline laparotomy $(n=43+n=9)$ 
Thomson 2015 (Continued)

Antibiotics: coamoxiclavulanic acid $1.2 \mathrm{~g} \mathrm{IV} \mathrm{every} 8 \mathrm{~h}$ or ciprofloxacin $500 \mathrm{mg}$ IV every $8 \mathrm{~h}$

Outcomes Intraoperative time; wound sepsis; reoperations; hospital stay; readmission; generalised contamination

Follow-up: 2 weeks after discharge
No appendectomy: none
Declaration of interest: the authors "declare no conflicts of interest or financial ties to disclose"
Funding: Department of Surgery, University of Witwatersrand (South Africa)
Preoperative imaging: "following ... radiological investigation..."
Analgesia requirements: not reported
Sample size calculation: not reported

\section{Risk of bias}

\begin{tabular}{lll}
\hline Bias & Authors' judgement & Support for judgement \\
\hline $\begin{array}{l}\text { Random sequence genera- } \\
\text { tion (selection bias) }\end{array}$ & Low risk & $\begin{array}{l}\text { "... randomization sequence generated by a computer random number gener- } \\
\text { ation software." }\end{array}$ \\
\hline $\begin{array}{l}\text { Allocation concealment } \\
\text { (selection bias) }\end{array}$ & Low risk & "Sequentially numbered non-transparent envelopes..." \\
\hline $\begin{array}{l}\text { Blinding of participants } \\
\begin{array}{l}\text { and personnel (perfor- } \\
\text { mance bias) }\end{array}\end{array}$ & Unclear risk & Not reported \\
All outcomes & \\
\hline
\end{tabular}

Blinding of outcome as- Unclear risk Apparently, only the pathologist was blinded.

sessment (detection bias)

All outcomes

\begin{tabular}{lll}
$\begin{array}{l}\text { Incomplete outcome data } \\
\text { (attrition bias) } \\
\begin{array}{l}\text { All outcomes } \\
\text { High risk }\end{array}\end{array}$ & $\begin{array}{l}\text { Thirty-one participants (21 and 10 in LA and OA) were excluded after randomi- } \\
\text { sation because of uncomplicated appendicitis, normal appendix or other diag- } \\
\text { nosis. } \\
\text { It was unclear if the two conversions were analysed according to the ITT princi- } \\
\text { ple. } \\
\text { No losses to follow-up occurred. }\end{array}$ \\
\hline $\begin{array}{l}\text { Selective reporting (re- } \\
\text { porting bias) }\end{array}$ & Unclear risk & $\begin{array}{l}\text { The study had } 5 \text { primary outcome measures, without any adjustment for mul- } \\
\text { tiple hypothesis testing. }\end{array}$ \\
\hline Other bias & Low risk & $\begin{array}{l}\text { "A senior team of consultant surgeons capable of completing both OA and LA } \\
\text { performed all the operations." }\end{array}$ \\
\hline
\end{tabular}

Tzovaras 2010

\begin{tabular}{ll}
\hline Methods & Randomised controlled trial \\
& Centres: one (Greece)
\end{tabular}


Tzovaras 2010 (Continued)

Participants
Participants: "older than 15 years with suspected acute appendicitis"

Gender: $0 \%$ females

Age: 26 and 22 years (median) in LA and OA group

Histology: $97 \%$ inflamed

Interventions

LA: three trocar technique (10-mm umbilical, two 5-mm trocars at the left lower quadrant); stump closure "with 2 absorbable endo loops" ( $n=72)$

OA: via McBurney incision $(n=75)$

Antibiotics: cefuroxim $750 \mathrm{mg}$ and metronidazole $500 \mathrm{mg}$ IV every $8 \mathrm{~h}$ for at least $24 \mathrm{~h}$

\section{Outcomes}

Primary outcome: hospital stay

Secondary outcomes: needs for analgesia postoperatively, 30-day morbidity, time until resumption of normal activities

Further outcomes: wound infection, time of surgery, mortality

Notes

Follow-up: "Outpatient follow-up was performed 7-10 days and 1 month postoperatively."

No appendectomy: not reported

Declaration of interest: The authors "have no conflicts of interest or financial ties to disclose".

Funding: not reported

Preoperative imaging: "Abdominal imaging with either ultrasonography or computed tomography (CT) scan were selectively - and rather exceptionally - performed at the discretion of the surgeon, primarily in equivocal cases (i.e. atypical presentation, older patients)."

Analgesia requirements: "Postoperative analgesia was given on demand, starting with NSAIDs and moving to opioids as required." No difference in consumption of analgesia between the groups

Sample size calculation: not reported

\section{Risk of bias}

\begin{tabular}{|c|c|c|}
\hline Bias & Authors' judgement & Support for judgement \\
\hline $\begin{array}{l}\text { Random sequence genera- } \\
\text { tion (selection bias) }\end{array}$ & Low risk & "was created by a computer-generated list in blocks of 20 patients." \\
\hline $\begin{array}{l}\text { Allocation concealment } \\
\text { (selection bias) }\end{array}$ & Low risk & $\begin{array}{l}\text { "numbered and sealed envelops were placed in the operating room and } \\
\text { opened only at patients' arrival" }\end{array}$ \\
\hline $\begin{array}{l}\text { Blinding of participants } \\
\text { and personnel (perfor- } \\
\text { mance bias) } \\
\text { All outcomes }\end{array}$ & Unclear risk & Not reported \\
\hline $\begin{array}{l}\text { Blinding of outcome as- } \\
\text { sessment (detection bias) } \\
\text { All outcomes }\end{array}$ & Unclear risk & Not reported \\
\hline $\begin{array}{l}\text { Incomplete outcome data } \\
\text { (attrition bias) } \\
\text { All outcomes }\end{array}$ & Low risk & $\begin{array}{l}\text { Conversions in the laparoscopic arm }(n=16 ; 22.2 \%) \text { were analysed according } \\
\text { to ITT. Data were apparently complete for all outcome variables. }\end{array}$ \\
\hline $\begin{array}{l}\text { Selective reporting (re- } \\
\text { porting bias) }\end{array}$ & Low risk & Data on main results appeared to be complete. \\
\hline
\end{tabular}


Tzovaras 2010 (Continued)

$\begin{array}{ll}\text { Other bias } \quad \text { Low risk } & \begin{array}{l}\text { "all the procedures were performed or supervised by a consultant surgeon ex- } \\ \text { perienced in both OA and LA". }\end{array}\end{array}$

Vallribera 2003

\begin{tabular}{|c|c|}
\hline Methods & $\begin{array}{l}\text { Randomised controlled trial } \\
\text { Centres: one (Spain) }\end{array}$ \\
\hline Participants & $\begin{array}{l}\text { Participants: adults with clinical and laboratory signs of acute appendicitis } \\
\text { Gender: } 34 \% \text { females } \\
\text { Age: } 30.3 \text { and } 34.7 \text { years (mean) in LA and OA } \\
\text { Histology: } 88 \% \text { inflamed }\end{array}$ \\
\hline Interventions & $\begin{array}{l}\text { LAP: a normal appendix was left when another definite cause of the participant's symptoms was found. } \\
\text { LA: three trocar technique; stump closure without GIA }(n=35) \\
\text { OA: via McBurney's incision }(n=30) \\
\text { Antibiotics: } 2 \text { g cefotixine IV }\end{array}$ \\
\hline Outcomes & Hospital stay; complication rates; duration of surgery; pain (VAS); quality of life \\
\hline Notes & $\begin{array}{l}\text { Follow-up: at weekly intervals until } 4 \text { weeks postoperatively } \\
\text { No appendectomy: was performed in } 3(9 \%) \text { laparoscopy participants, in whom another diagnosis was } \\
\text { made } \\
\text { Declaration of interest: not reported } \\
\text { Funding: not reported } \\
\text { Preoperative imaging: not reported } \\
\text { Analgesia requirements: not reported } \\
\text { Sample size calculation: adequately reported }\end{array}$ \\
\hline
\end{tabular}

\section{Risk of bias}

\begin{tabular}{|c|c|c|}
\hline Bias & Authors' judgement & Support for judgement \\
\hline $\begin{array}{l}\text { Random sequence genera- } \\
\text { tion (selection bias) }\end{array}$ & Low risk & "using a random numbers table" \\
\hline $\begin{array}{l}\text { Allocation concealment } \\
\text { (selection bias) }\end{array}$ & Low risk & "sealed numbered envelopes" \\
\hline $\begin{array}{l}\text { Blinding of participants } \\
\text { and personnel (perfor- } \\
\text { mance bias) } \\
\text { All outcomes }\end{array}$ & Unclear risk & Not reported \\
\hline $\begin{array}{l}\text { Blinding of outcome as- } \\
\text { sessment (detection bias) } \\
\text { All outcomes }\end{array}$ & Unclear risk & Not reported \\
\hline $\begin{array}{l}\text { Incomplete outcome data } \\
\text { (attrition bias) } \\
\text { All outcomes }\end{array}$ & Low risk & $\begin{array}{l}\text { Analyses were done according to ITT and thus included converted cases ( } \mathrm{n}=3 \text {; } \\
9 \% \text { ) and participants with another diagnosis. Completeness of follow-up was } \\
\text { not described in detail but losses to follow-up appeared to be negligible. }\end{array}$ \\
\hline
\end{tabular}


Vallribera 2003 (Continued)

Selective reporting (re- Unclear risk No distinction between primary and secondary outcome
porting bias)

Other bias

Low risk

All surgeons had experience with more than 50 laparoscopic cholecystectomies.

Van Dalen 2003

\begin{tabular}{|c|c|}
\hline Methods & $\begin{array}{l}\text { Randomised controlled trial } \\
\text { Centres: one (New Zealand) }\end{array}$ \\
\hline Participants & $\begin{array}{l}\text { Participants: women with "a clinical diagnosis of acute appendicitis". } \\
\text { Gender: } 100 \% \text { females } \\
\text { Age: between } 24 \text { and } 22 \text { years (median) in LA and OA } \\
\text { Histology: } 75 \% \text { inflamed }\end{array}$ \\
\hline Interventions & $\begin{array}{l}\text { LAP: diagnostic laparoscopy followed by OA only when acute appendicitis was visible }(n=32) \\
\text { OPEN: technique not described }(n=31) \\
\text { Antibiotics: not reported }\end{array}$ \\
\hline Outcomes & Hospital stay; complication rates; duration of surgery \\
\hline Notes & $\begin{array}{l}\text { Follow-up: apparently within hospital stay, but seven participants with their appendix still in situ were } \\
\text { reviewed after } 10 \text { years. } \\
\text { No appendectomy: appendix left in situ in seven participants. } \\
\text { Declaration of interest: not reported } \\
\text { Funding: not reported } \\
\text { Preoperative imaging: not reported } \\
\text { Analgesia requirements: not reported } \\
\text { Sample size calculation: not reported }\end{array}$ \\
\hline
\end{tabular}

Risk of bias

\begin{tabular}{lll}
\hline Bias & Authors' judgement & Support for judgement \\
\hline $\begin{array}{l}\text { Random sequence genera- } \\
\text { tion (selection bias) }\end{array}$ & Unclear risk & "Patients were randomized..." \\
\hline $\begin{array}{l}\text { Allocation concealment } \\
\text { (selection bias) }\end{array}$ & Low risk & "... by closed-envelope system." \\
\hline $\begin{array}{l}\text { Blinding of participants } \\
\begin{array}{l}\text { and personnel (perfor- } \\
\text { mance bias) }\end{array}\end{array}$ & Unclear risk & Not reported \\
$\begin{array}{l}\text { All outcomes } \\
\text { Blinding of outcome as- } \\
\text { sessment (detection bias) } \\
\text { All outcomes }\end{array}$ & Unclear risk & Not reported \\
\hline
\end{tabular}


Van Dalen 2003 (Continued)

Incomplete outcome data Low risk There were no conversions. Analyses were apparently done following the ITT (attrition bias) principle. Losses to follow-up were not described in detail but appeared to be All outcomes negligible with regard to the short-term results.

\begin{tabular}{lll}
$\begin{array}{l}\text { Selective reporting (re- } \\
\text { porting bias) }\end{array}$ & Unclear risk & No distinction between primary and secondary outcome \\
\hline Other bias & Unclear risk & $\begin{array}{l}\text { Operations were performed by two registrars, but their expertise was not de- } \\
\text { scribed. }\end{array}$
\end{tabular}

\section{Wei 2010}

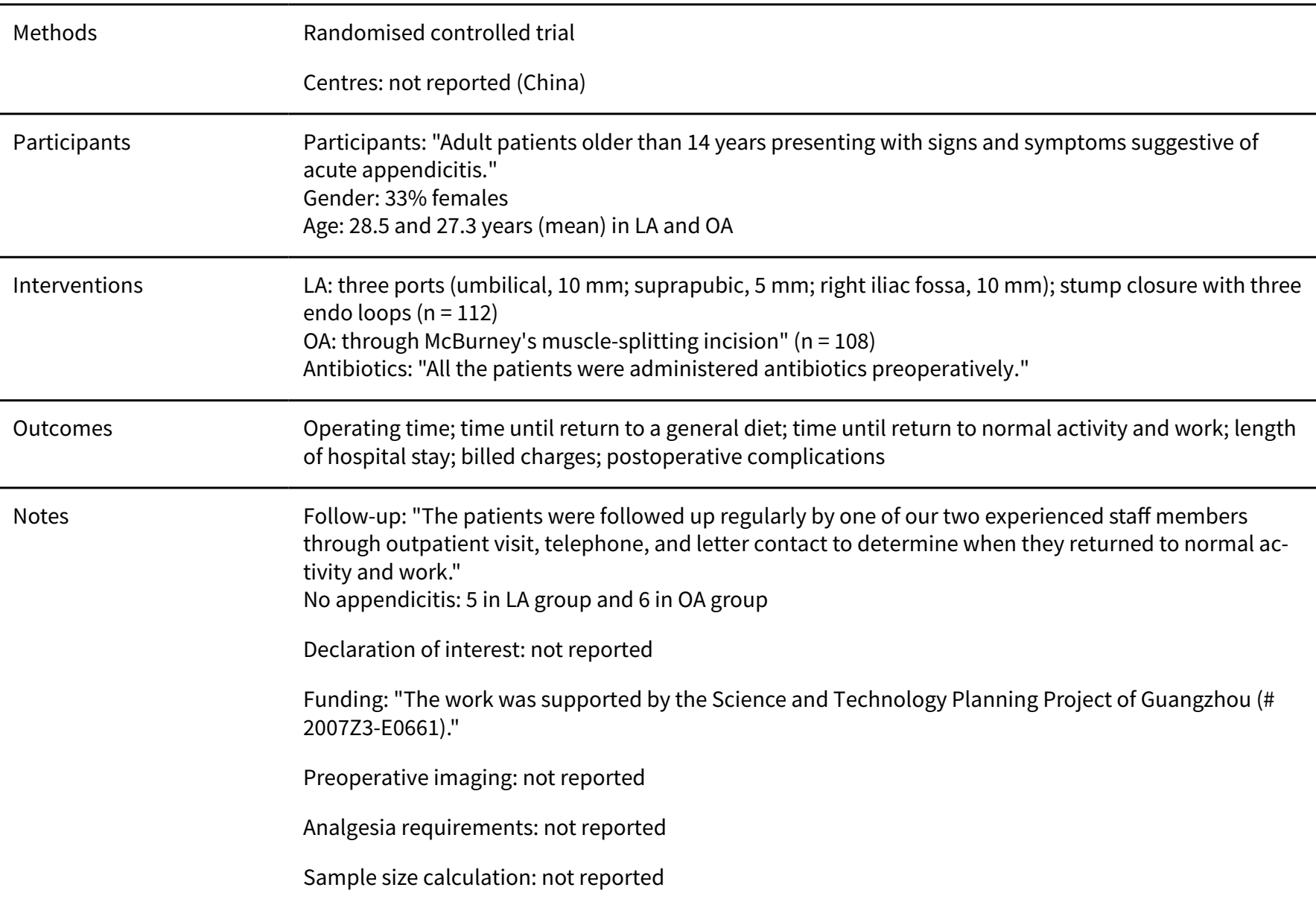

\section{Risk of bias}

\begin{tabular}{lll}
\hline Bias & Authors' judgement & Support for judgement \\
\hline $\begin{array}{l}\text { Random sequence genera- } \\
\text { tion (selection bias) }\end{array}$ & Unclear risk & "[Patients] were randomized to OA and LA groups." \\
\hline $\begin{array}{l}\text { Allocation concealment } \\
\text { (selection bias) }\end{array}$ & Unclear risk & Not reported \\
\hline $\begin{array}{l}\text { Blinding of participants } \\
\text { and personnel (perfor- } \\
\text { mance bias) }\end{array}$ & Unclear risk & Not reported \\
\hline
\end{tabular}


Wei 2010 (Continued)

All outcomes

\begin{tabular}{lll}
$\begin{array}{l}\text { Blinding of outcome as- } \\
\text { sessment (detection bias) } \\
\text { All outcomes }\end{array}$ & Unclear risk & Not reported \\
\hline $\begin{array}{l}\text { Incomplete outcome data } \\
\text { (attrition bias) }\end{array}$ & Low risk & $\begin{array}{l}\text { No conversion were reported. Follow-up data appeared to be similarly com- } \\
\text { pll outcomes in both groups. }\end{array}$
\end{tabular}

All outcomes

Selective reporting (re- Unclear risk $\quad$ No distinction between primary and secondary outcome
porting bias)
porting bias)

\begin{tabular}{ll}
\hline Other bias $\quad$ Low risk & $\begin{array}{l}\text { "All the operations were performed by the same operation team experienced } \\
\text { in laparoscopic and open surgical techniques." }\end{array}$ \\
\hline
\end{tabular}

Williams 1996

Rethods
Centres: one (USA)
Comment: The article actually described a nonrandomised and a randomised trial.

\begin{tabular}{|c|c|}
\hline Participants & $\begin{array}{l}\text { Participants: "with a preoperative diagnosis if acute appendicitis" } \\
\text { Gender: } 24 \% \text { females } \\
\text { Age: } 28 \text { and } 26 \text { years (mean) in LA and OA } \\
\text { Histology: } 78 \% \text { inflamed }\end{array}$ \\
\hline Interventions & $\begin{array}{l}\text { LA: "The technique for appendectomy, either open or laparoscopic, was left entirely to the surgeon's } \\
\text { discretion."Stump closure was done with a disposable GIA stapler }(n=19) \\
\text { OA: not described }(n=18) \\
\text { Antibiotics: not reported }\end{array}$ \\
\hline Outcomes & $\begin{array}{l}\text { Hospital stay; return to normal activities; return to work; complication rates; duration of operation; } \\
\text { analgesic drugs; costs; participant satisfaction rate }\end{array}$ \\
\hline \multirow[t]{6}{*}{ Notes } & $\begin{array}{l}\text { Follow-up: by telephone interview } 1 \text { month after surgery } \\
\text { No appendectomy: not reported }\end{array}$ \\
\hline & Declaration of interest: not reported \\
\hline & Funding: not reported \\
\hline & Preoperative imaging: not reported \\
\hline & $\begin{array}{l}\text { Analgesia requirements: "... duration of postoperative intramuscular (IM) or intravenous (IV) analgesic } \\
\text { administration ... were recorded." No significant difference in IM analgesia. The results of IV analgesia } \\
\text { were not reported. }\end{array}$ \\
\hline & Sample size calculation: not reported \\
\hline
\end{tabular}

\section{Risk of bias}

\begin{tabular}{lll}
\hline Bias & Authors' judgement & Support for judgement \\
\hline $\begin{array}{l}\text { Random sequence genera- } \\
\text { tion (selection bias) }\end{array}$ & Unclear risk & "Patients were randomised..." \\
\hline \hline
\end{tabular}


Williams 1996 (Continued)

\begin{tabular}{lll}
$\begin{array}{l}\text { Allocation concealment } \\
\text { (selection bias) }\end{array}$ & Unclear risk & Not reported \\
\hline $\begin{array}{l}\text { Blinding of participants } \\
\text { and personnel (perfor- } \\
\text { mance bias) }\end{array}$ & Unclear risk & Not reported \\
All outcomes & &
\end{tabular}

\begin{tabular}{|c|c|c|}
\hline $\begin{array}{l}\text { Blinding of outcome as- } \\
\text { sessment (detection bias) } \\
\text { All outcomes }\end{array}$ & Unclear risk & Not reported \\
\hline
\end{tabular}

\begin{tabular}{|c|c|c|}
\hline $\begin{array}{l}\text { Incomplete outcome data } \\
\text { (attrition bias) } \\
\text { All outcomes }\end{array}$ & High risk & $\begin{array}{l}\text { Converted cases ( } \mathrm{n}=2 ; 10 \%) \text { were not analysed according to ITT: "The statis- } \\
\text { tical analysis presented here excludes those two cases, but even inclusion of } \\
\text { these two cases does not change any of the significant results." } \\
\text { Quotation: "The postoperative follow-up and 1-month postoperative inter- } \\
\text { view data were incomplete." }\end{array}$ \\
\hline $\begin{array}{l}\text { Selective reporting (re- } \\
\text { porting bias) }\end{array}$ & Unclear risk & No distinction between primary and secondary outcome \\
\hline Other bias & Unclear risk & The surgeon's skills were not described. \\
\hline
\end{tabular}

\section{Witten 1998}

\begin{tabular}{|c|c|}
\hline Methods & $\begin{array}{l}\text { Randomised controlled trial } \\
\text { Centres: one (Germany) }\end{array}$ \\
\hline Participants & $\begin{array}{l}\text { Participants: patients older } 6 \text { yr with a clinical diagnosis of acute appendicitis } \\
\text { Gender: } 41 \% \text { females. } \\
\text { Age: } 29 \text { and } 26 \text { years (median) in LA and OA } \\
\text { Histology: } 87 \% \text { inflamed }\end{array}$ \\
\hline Interventions & $\begin{array}{l}\text { LA: three trocar technique }(12,10 \text {, and } 5 \mathrm{~mm}) \text {; stump closure with stapling device }(\mathrm{n}=100) \\
\text { OA: via McBurney approach }(\mathrm{n}=100) \\
\text { Antibiotics: cefuroxime three doses of } 1.5 \mathrm{mg} \text { and metronidazole } 2 \text { doses of } 0.5 \mathrm{mg} \text { (children received } \\
\text { adapted dosage) }\end{array}$ \\
\hline Outcomes & $\begin{array}{l}\text { Hospital stay; return to normal activities; complication rates; duration of operation; pain (VAS); pain } \\
\text { medication }\end{array}$ \\
\hline \multirow[t]{6}{*}{ Notes } & $\begin{array}{l}\text { Follow-up: within hospital stay } \\
\text { No appendectomy: was necessary in one conventional and two laparoscopic cases }\end{array}$ \\
\hline & Declaration of interest: not reported \\
\hline & Funding: not reported \\
\hline & Preoperative imaging: not reported \\
\hline & Analgesia requirements: not standardised \\
\hline & Sample size calculation: not reported \\
\hline
\end{tabular}

\section{Risk of bias}


Witten 1998 (Continued)

\section{Bias Authors' judgement Support for judgement}

Random sequence genera- Low risk tion (selection bias)

A electronic random generator was described ("Die Lose waren durchgehend nummeriert, die Zuteilung mit Hilfe eines elektronischen Zufallsgenerators erfolgt.")

\begin{tabular}{ll}
\hline $\begin{array}{l}\text { Allocation concealment } \\
\text { (selection bias) }\end{array}$ & Low risk \\
& $\begin{array}{l}\text { Sealed envelopes were described ("Alle Lose befanden sich in versiegelten } \\
\text { zugeteilten Operationsverfahren hatte, bis die Operationsindikation fest- } \\
\text { stand.") }\end{array}$ \\
\hline
\end{tabular}

Blinding of participants High risk No blinding
and personnel (perfor-
mance bias)
All outcomes

Blinding of outcome as- High risk No blinding

sessment (detection bias)

All outcomes

$\begin{array}{ll}\begin{array}{l}\text { Incomplete outcome data } \\ \text { (attrition bias) }\end{array} & \begin{array}{l}\text { Conversions }(n=5 ; 5 \%) \text { and those without appendectomy were analysed ac- } \\ \text { cording to ITT. However, the analyses on pain failed to include the no appen- } \\ \text { All outcomes }\end{array} \\ & \text { fectomy cases. } \\ & \text { Follow-up within hospital stay was complete for all enrolled participants. }\end{array}$

\begin{tabular}{lll}
\hline $\begin{array}{l}\text { Selective reporting (re- } \\
\text { porting bias) }\end{array}$ & Unclear risk & No distinction between primary and secondary outcome \\
\hline Other bias & High risk & LA was more likely to be performed by a senior surgeon. \\
\hline
\end{tabular}

\section{Yeung 1997}

\begin{tabular}{|c|c|}
\hline Methods & $\begin{array}{l}\text { Randomised controlled trial } \\
\text { Centres: one (China) }\end{array}$ \\
\hline Participants & $\begin{array}{l}\text { Participants: children (aged } 4 \text { to } 15 \text { years) with acute appendicitis } \\
\text { Gender: not mentioned } \\
\text { Age: not further specified } \\
\text { Histology: not given }\end{array}$ \\
\hline Interventions & $\begin{array}{l}\text { LA: } \text { not specified }(n=91) \\
\text { OA: not specified }(n=90) \\
\text { Antibiotics: not reported }\end{array}$ \\
\hline Outcomes & Hospital stay; return to normal activities; complication rates; duration of operation \\
\hline \multirow[t]{4}{*}{ Notes } & $\begin{array}{l}\text { Follow-up: not reported } \\
\text { Conversions: } n=11(12 \%) \text { analysed by ITT } \\
\text { No appendectomy: not reported }\end{array}$ \\
\hline & Declaration of interest: not reported \\
\hline & Funding: not reported \\
\hline & Preoperative imaging: not reported \\
\hline
\end{tabular}


Yeung 1997 (Continued)

Analgesia requirements: not reported

Sample size calculation: not reported

\section{Risk of bias}

\begin{tabular}{|c|c|c|}
\hline Bias & Authors' judgement & Support for judgement \\
\hline $\begin{array}{l}\text { Random sequence genera- } \\
\text { tion (selection bias) }\end{array}$ & Unclear risk & "participants were randomised..." \\
\hline $\begin{array}{l}\text { Allocation concealment } \\
\text { (selection bias) }\end{array}$ & Low risk & $\begin{array}{l}\text { The primary author stated that the trial was "properly randomised [...] by } \\
\text { drawing sealed envelopes". (written communication by Dr. Yeung dated July } \\
\text { 14th, 1999) }\end{array}$ \\
\hline $\begin{array}{l}\text { Blinding of participants } \\
\text { and personnel (perfor- } \\
\text { mance bias) } \\
\text { All outcomes }\end{array}$ & High risk & No blinding \\
\hline $\begin{array}{l}\text { Blinding of outcome as- } \\
\text { sessment (detection bias) } \\
\text { All outcomes }\end{array}$ & High risk & No blinding \\
\hline $\begin{array}{l}\text { Incomplete outcome data } \\
\text { (attrition bias) } \\
\text { All outcomes }\end{array}$ & Unclear risk & Not reported \\
\hline $\begin{array}{l}\text { Selective reporting (re- } \\
\text { porting bias) }\end{array}$ & Unclear risk & No distinction between primary and secondary outcome \\
\hline Other bias & Low risk & $\begin{array}{l}\text { "A laparoscopic appendectomy on-call team [] formed by four la- } \\
\text { paroscopy-trained staff pediatric surgeons was established specifically for this } \\
\text { study." (written communication by Dr. Yeung dated July 14th, 1999) }\end{array}$ \\
\hline
\end{tabular}

Yin 1996

\begin{tabular}{ll} 
Methods & Randomised controlled trial \\
& Centres: one (Taiwan) \\
\hline Participants & $\begin{array}{l}\text { Participants: patients older than } 12 \text { years with a preoperative diagnosis of acute appendicitis } \\
\text { Gender: } 47 \% \text { females } \\
\text { Age: } 36 \text { years (unclear if mean or median) } \\
\text { Histology: about } 80 \% \text { inflamed }\end{array}$ \\
\hline Interventions & $\begin{array}{l}\text { necessary, the surgeons used another } 5 \mathrm{~mm} \text { port in left lower abdomen for better manipulation. Stump } \\
\text { dissection after application of loop ligatures ( } \mathrm{n}=30 \text { ) }\end{array}$ \\
OA: generally McBurney incision, except two participants were operated on through low middle inci- \\
sion for preoperative diagnosis of diffuse peritonitis ( $\mathrm{n}=40)$. \\
Antibiotics: cephalosporin
\end{tabular}

Outcomes

Operation time; hospital stay; days before diet; analgesic doses; wound infection; time until return to normal activities 
Yin 1996 (Continued)

Notes
Follow-up: on seventh postoperative day and then weekly for three consecutive weeks by face-to-face and phone contact

No appendectomy: not reported

Declaration of interest: not reported

Funding: "This study was supported by the grant of the Tzu Chi Medical Research Center under the auspices of Buddhist Tzu Chi General Hospital."

Preoperative imaging: not reported

Analgesia requirements: not specified, there was no difference in analgesic doses $(P=0.69)$

Sample size calculation: not reported

\section{Risk of bias}

\begin{tabular}{|c|c|c|}
\hline Bias & Authors' judgement & Support for judgement \\
\hline $\begin{array}{l}\text { Random sequence genera- } \\
\text { tion (selection bias) }\end{array}$ & Low risk & "using random number table" \\
\hline $\begin{array}{l}\text { Allocation concealment } \\
\text { (selection bias) }\end{array}$ & Low risk & "sealed envelope system" \\
\hline $\begin{array}{l}\text { Blinding of participants } \\
\text { and personnel (perfor- } \\
\text { mance bias) } \\
\text { All outcomes }\end{array}$ & Unclear risk & $\begin{array}{l}\text { "The medical personnel who took part in postoperative care did not know } \\
\text { which patients were designed for the trial. They cared for the patients as usual } \\
\text { and the operative team workers did not get involved in postoperative care in } \\
\text { order to avoid bias." }\end{array}$ \\
\hline $\begin{array}{l}\text { Blinding of outcome as- } \\
\text { sessment (detection bias) } \\
\text { All outcomes }\end{array}$ & Unclear risk & Not reported \\
\hline $\begin{array}{l}\text { Incomplete outcome data } \\
\text { (attrition bias) } \\
\text { All outcomes }\end{array}$ & Low risk & $\begin{array}{l}\text { Data appeared to be complete. No conversions or other changes in surgical } \\
\text { procedure stated }\end{array}$ \\
\hline $\begin{array}{l}\text { Selective reporting (re- } \\
\text { porting bias) }\end{array}$ & Unclear risk & No distinction between primary and secondary outcome \\
\hline Other bias & Low risk & $\begin{array}{l}\text { "Initially, a pilot study was done in } 10 \text { cases for gaining a standard surgical } \\
\text { technique and better cooperation among the surgical team workers. The same } \\
\text { surgical team involved in operating all the patients in both groups. Each and } \\
\text { every case was operated by a chief resident or/ and attending staff." }\end{array}$ \\
\hline
\end{tabular}

\section{Yu 2016}

\begin{tabular}{ll}
\hline Methods & Randomised controlled trial \\
& Centres: one (China) \\
\hline Participants & Participants: Children "having appendicitis according to diagnostic standard of appendicitis released \\
by WHO" & Gender: $59 \%$ females \\
Age: 7.9 and 7.2 years (mean) in LA and OA \\
Histology: $100 \%$ inflamed \\
\hline
\end{tabular}


Yu 2016 (Continued)

Interventions
LA: three trocar technique $(5,3$, and $5 \mathrm{~mm})$; appendix root was double ligatured with absorbable clips $(\mathrm{n}=130)$

OA: McBurney incision $(n=130)$

Antibiotics:

LA: "Abdominal cavity was washed constantly until the liquid become clear."

OA: "Abdominal cavity was washed by metronidazole."

Postoperative administration of antibiotics not specified

Duration of operation; amount of bleeding; time until out of bed; time to take food; exhaust time; catheterisation time; application time of antibiotics; usage of analgesics; drainage time; incidence of complications

Follow-up: not reported
No appendectomy: not reported
Declaration of interest: "All authors declared there was no conflict interests involved."
Funding: "Grant Support \& Financial Disclosures: None."
Preoperative imaging: not reported
Analgesia requirements: "Usage of analgesic in laparoscopic appendectomy group was lower than
open appendectomy group, but no significant difference was observed."
Sample size calculation: not reported

\section{Risk of bias}

\begin{tabular}{lll}
\hline Bias & Authors' judgement & Support for judgement \\
\hline $\begin{array}{l}\text { Random sequence genera- } \\
\text { tion (selection bias) }\end{array}$ & Unclear risk & $\begin{array}{l}\text { "All patients were randomly and evenly divided into laparoscopic appendecto- } \\
\text { my group and open appendectomy group." }\end{array}$ \\
\hline $\begin{array}{l}\text { Allocation concealment } \\
\text { (selection bias) }\end{array}$ & Unclear risk & Not reported \\
\hline $\begin{array}{l}\text { Blinding of participants } \\
\text { and personnel (perfor- } \\
\text { mance bias) } \\
\text { All outcomes }\end{array}$ & Unclear risk & Not reported \\
\hline $\begin{array}{l}\text { Blinding of outcome as- } \\
\text { sessment (detection bias) } \\
\text { All outcomes }\end{array}$ & Unclear risk & Not reported \\
\hline $\begin{array}{l}\text { Incomplete outcome data } \\
\text { (attrition bias) }\end{array}$ & Low risk & $\begin{array}{l}\text { Dll outcomes } \\
\text { pelective reporting (re- }\end{array}$ \\
$\begin{array}{l}\text { porting bias) } \\
\text { Other bias }\end{array}$ & Unclear risk & No distinction between primary and secondary outcome \\
\hline
\end{tabular}


Zhang 1998

\begin{tabular}{|c|c|}
\hline Methods & $\begin{array}{l}\text { Randomised controlled trial } \\
\text { Centres: one (?) (China) }\end{array}$ \\
\hline Participants & $\begin{array}{l}\text { Participants: patients older than } 16 \text { yr with a clinical diagnosis of acute appendicitis } \\
\text { Gender: } 41 \% \text { females } \\
\text { Age: } 31.3 \text { and } 35.8 \text { years (mean) in LA and OA } \\
\text { Histology: } 100 \% \text { inflamed }\end{array}$ \\
\hline Interventions & $\begin{array}{l}\text { LA: Trocar sizes and stump closure technique not specified }(n=50) \\
\text { OA: described as "mini-incision appendectomy" }(2.5-4.5 \mathrm{~cm}) \text {. All operations were made under local } \\
\text { anaesthesia }(n=53) \\
\text { Antibiotics: Amoxicillin and metronidazole (?) IV }\end{array}$ \\
\hline Outcomes & $\begin{array}{l}\text { Hospital stay; complication rates; duration of operation; duration of analgesic drug use; length of inci- } \\
\text { sion; intraoperative blood loss }\end{array}$ \\
\hline \multirow[t]{6}{*}{ Notes } & $\begin{array}{l}\text { Follow-up: not reported } \\
\text { No appendectomy: not reported }\end{array}$ \\
\hline & Declaration of interest: not reported \\
\hline & Funding: not reported \\
\hline & Preoperative imaging: not reported \\
\hline & Analgesia requirements: not reported \\
\hline & Sample size calculation: not reported \\
\hline
\end{tabular}

\section{Risk of bias}

\begin{tabular}{|c|c|c|}
\hline Bias & Authors' judgement & Support for judgement \\
\hline $\begin{array}{l}\text { Random sequence genera- } \\
\text { tion (selection bias) }\end{array}$ & Low risk & "randomisation from random numbers table" \\
\hline $\begin{array}{l}\text { Allocation concealment } \\
\text { (selection bias) }\end{array}$ & Unclear risk & Not reported \\
\hline $\begin{array}{l}\text { Blinding of participants } \\
\text { and personnel (perfor- } \\
\text { mance bias) } \\
\text { All outcomes }\end{array}$ & Unclear risk & Not reported \\
\hline $\begin{array}{l}\text { Blinding of outcome as- } \\
\text { sessment (detection bias) } \\
\text { All outcomes }\end{array}$ & Unclear risk & Not reported \\
\hline $\begin{array}{l}\text { Incomplete outcome data } \\
\text { (attrition bias) } \\
\text { All outcomes }\end{array}$ & Unclear risk & $\begin{array}{l}\text { No reporting of the number and reasons for exclusions or completeness of fol- } \\
\text { low-up }\end{array}$ \\
\hline $\begin{array}{l}\text { Selective reporting (re- } \\
\text { porting bias) }\end{array}$ & Unclear risk & No distinction between primary and secondary outcome \\
\hline Other bias & Low risk & $\begin{array}{l}\text { The study started after more than } 3 \text { years of experience with laparoscopic } \\
\text { surgery. }\end{array}$ \\
\hline
\end{tabular}


Özmen 1999

\begin{tabular}{|c|c|}
\hline Methods & $\begin{array}{l}\text { Randomised controlled trial } \\
\text { Centres: one (Turkey) }\end{array}$ \\
\hline Participants & $\begin{array}{l}\text { Participants: patients with clinical suspicion of acute appendicitis } \\
\text { Gender: } 54 \% \text { females } \\
\text { Age: } 23 \text { and } 28 \text { years (mean) in LA and OA } \\
\text { Histology: } 90 \% \text { inflamed }\end{array}$ \\
\hline Interventions & $\begin{array}{l}\text { LA: three trocar technique, stump closure with endo loop }(n=35) \\
\text { OA: via muscle-splitting incision }(n=35) \\
\text { Antibiotics: "All patients were given routine prophylactic intravenous antibiotics on induction of } \\
\text { anaesthesia". Type of drug not specified }\end{array}$ \\
\hline Outcomes & Hospital stay; complication rates; duration of operation; cosmesis \\
\hline \multirow[t]{6}{*}{ Notes } & $\begin{array}{l}\text { Follow-up: "All patients were seen } 1 \text { and } 4 \text { weeks after discharge." } \\
\text { No appendectomy: not reported }\end{array}$ \\
\hline & Declaration of interest: not reported \\
\hline & Funding: not reported \\
\hline & Preoperative imaging: not reported \\
\hline & Analgesia requirements: not reported \\
\hline & Sample size calculation: not reported \\
\hline
\end{tabular}

\section{Risk of bias}

\begin{tabular}{|c|c|c|}
\hline Bias & Authors' judgement & Support for judgement \\
\hline $\begin{array}{l}\text { Random sequence genera- } \\
\text { tion (selection bias) }\end{array}$ & Unclear risk & "Patients were randomised..." \\
\hline $\begin{array}{l}\text { Allocation concealment } \\
\text { (selection bias) }\end{array}$ & Unclear risk & Not reported \\
\hline $\begin{array}{l}\text { Blinding of participants } \\
\text { and personnel (perfor- } \\
\text { mance bias) } \\
\text { All outcomes }\end{array}$ & Unclear risk & Not reported \\
\hline $\begin{array}{l}\text { Blinding of outcome as- } \\
\text { sessment (detection bias) } \\
\text { All outcomes }\end{array}$ & Unclear risk & Not reported \\
\hline $\begin{array}{l}\text { Incomplete outcome data } \\
\text { (attrition bias) } \\
\text { All outcomes }\end{array}$ & Unclear risk & $\begin{array}{l}\text { No reporting of the number and reasons for exclusions or completeness of fol- } \\
\text { low-up }\end{array}$ \\
\hline $\begin{array}{l}\text { Selective reporting (re- } \\
\text { porting bias) }\end{array}$ & Unclear risk & No distinction between primary and secondary outcome \\
\hline Other bias & Unclear risk & Surgeons "with a wide range of experience" performed the study operations. \\
\hline
\end{tabular}


CRP: c-reactive protein

CT: computed tomography

FLACC: face, legs, activitiy, cry, consolability scale

GIQLI: gastrointestinal quality of life index

IM: intramuscular

ITT: Intention to treat

IV: intravenously

LA: laparoscopic appendectomy

LAP: diagnostic laparoscopy (eventually followed by OA or LA)

OA: open appendectomy

OPEN: open exploration (eventually followed by OA)

PCA: patient controlled analgesia

PP: per protocol

QoL: quality of life

VAS: visual analog scale

WHO: world health organization

Characteristics of excluded studies [ordered by study ID]

\begin{tabular}{ll}
\hline Study & Reason for exclusion \\
\hline Ablassmaier 1996 & $\begin{array}{l}\text { Choice of surgical procedure depended on the availability of surgeon and technical equipment. } \\
\text { Thus, the study was nonrandomised, although an accompanying editorial falsely called the study } \\
\text { randomised. }\end{array}$ \\
\hline Ali 2017 & $\begin{array}{l}\text { In an email, Prof. Akhtar stated that "randomization was done by finding from already list of com- } \\
\text { puter generated number, as to which group patient will be assigned." Thus, the study was ran- } \\
\text { domised, but without allocation concealment.. }\end{array}$
\end{tabular}

Almond 2004

Choice of surgical procedure depended on the surgeon. Three of twelve surgeons routinely performed LA.

Alvarado-Aparicio 2003 In the abstract, the study was said to be randomised, but retrospectively. Obviously, the study was not randomised.

\begin{tabular}{ll}
\hline Askarpour 2012 & $\begin{array}{l}\text { In an email dated October 15th, 2012, the first author stated: "We used simple alternation. [...] Pa- } \\
\text { tients were placed in the OA and LA group alternatively." Thus, the study was pseudo-randomised. }\end{array}$ \\
\hline Azaro 1999 & The study was not randomised. \\
\hline Barrat 1998 & The study was retrospective. \\
\hline Borgstein 1997 & The study had no control group. \\
\hline Buanes 1993 & $\begin{array}{l}\text { Choice of surgical procedure depended on the availability of surgeon and technical equipment. } \\
\text { "Randomization after informed consent was not possible." }\end{array}$ \\
\hline Busch 2011 & $\begin{array}{l}\text { The study was not randomised, as surgical technique was selected "according to departmental pol- } \\
\text { icy and surgeon preference". }\end{array}$ \\
\hline Champault 1993 & \begin{tabular}{l} 
Choice of surgical procedure depended on the availability of surgeon and technical equipment. \\
\hline Chen 2007
\end{tabular} \\
$\begin{array}{l}\text { Although the abstract claimed that group allocation was "randomly", the study was judged to be } \\
\text { pseudo-randomised by the review authors. Consecutive participants received numbers (1 to 200) } \\
\text { and were then allocated into the groups according to number (odd versus even). This mechanism } \\
\text { produces alternation but no formal randomisation. }\end{array}$
\end{tabular}




\begin{tabular}{ll}
\hline Study & Reason for exclusion \\
\hline Chiarugi 1996 & $\begin{array}{l}\text { The study was pseudo-randomised, and therefore without allocation concealment: "The patients } \\
\text { were allocated to the two groups depending on whether the date of admission was an odd or even } \\
\text { number." }\end{array}$
\end{tabular}

\begin{tabular}{ll} 
Darzi 1994 & "There was no formal randomization in the study because of instrument and surgeon availability." \\
\hline Decadt 1999 & This randomised study compared early laparoscopy versus observation. \\
\hline Deutsch 1982 & The study had no control group. \\
\hline Fang 2006 & $\begin{array}{l}\text { Because of the imbalance in group size (LA, } \mathrm{n}=53, \text { versus OA, } \mathrm{n}=77 \text { ) the study was considered to } \\
\text { be nonrandomised. }\end{array}$ \\
\hline Gilchrist 1992 & $\begin{array}{l}\text { Study was not randomised: "Selection for OA or LA was done by surgeon availability, according to } \\
\text { the staff paediatric surgeon on-call schedule. When the laparoscopy-trained surgeon was available, } \\
\text { all patients (regardless of the severity of their illness) were offered the laparoscopic procedure." }\end{array}$
\end{tabular}

Hanning 2006

Hay 1998
Allocation was pseudo-randomised since parents decided the operative procedure.

In an email, Prof. Hay stated that "an open list of random codes" was used. Thus, the study was randomised, but without allocation concealment. As the uneven distribution between the group suggested, the study was not analysed according to intention-to-treat.

\begin{tabular}{ll}
\hline Herman 2000 & In an email, the first author stated that the study was retrospective. \\
\hline Herman 2003 & The study was not randomised. \\
\hline Hill 1991 & $\begin{array}{l}\text { "Selection of the procedure in these patients depended on the availability of staff, equipment and } \\
\text { theatre time." (One year later, the same group performed a randomised trial, published by Attwood } \\
\text { in 1992.) }\end{array}$
\end{tabular}

In an email dated December 8th, 2014, the first author stated: "We used simple alternation tech-
niques". Thus, the study was pseudo-randomised.

\begin{tabular}{ll}
\hline Karaorman 1994 & $\begin{array}{l}\text { Choice of surgical procedure depended on participants' symptoms. Cases with unclear symptoms } \\
\text { underwent laparoscopy, if a skilled surgeon was available. }\end{array}$ \\
\hline Klima 1998 & $\begin{array}{l}\text { In the } 1998 \text { publication, a randomised clinical trial with three different techniques of appendix } \\
\text { stump closure was reported. In the } 1996 \text { publication, these three laparoscopic groups were com- } \\
\text { pared against conventional appendectomy, but the choice between open or laparoscopic tech- } \\
\text { nique depended on the availability of laparoscopically experienced surgeons. }\end{array}$ \\
\hline Kollias 1994 & $\begin{array}{l}\text { "True randomization was not formally obtained", because patients "were prospectively assigned to } \\
\text { either open appendicectomy (OA) if admitted under Surgical Unit B or laparoscopic appendicecto- } \\
\text { my (LA) if admitted under Surgical Unit A." }\end{array}$ \\
\hline
\end{tabular}

Koluh 2010 In an email dated October 18th, 2012, the first author stated: "A simple randomization of the-
ABABAB type was used." Thus, the study was pseudo-randomised.

Konstadoulakis $2006 \quad$ Comparison of two open techniques.

Kotlobovskii 2003

The study was excluded because it had included children with appendicular peritonitis. Average disease duration was over two days, and postoperative admission to the intensive care unit was necessary in most cases. The study still seems to be important, since it was obviously randomised, although the method of randomisation was not described. 


\begin{tabular}{|c|c|}
\hline Study & Reason for exclusion \\
\hline Kum 1993b & The study was not randomised, because surgeon availability determined choice of procedure. \\
\hline Kumar 2004 & Comparison of open techniques. \\
\hline Lamparelli 2000 & $\begin{array}{l}\text { Control participants were "under the care of the other surgical teams in our unit during the study } \\
\text { period". }\end{array}$ \\
\hline Lansdown 1993 & Study was not randomised. Control group was matched. \\
\hline Lau 2005 & Comparison between needlescopic appendectomy and conventional laparoscopic appendectomy. \\
\hline Li 2005 & $\begin{array}{l}\text { Study was not randomised: "For every patient, the patients or their parents were the ones to } \\
\text { choose the approach,..." (LA, } n=69 \text {, versus } O A, n=91) \text {. }\end{array}$ \\
\hline Lujan Mompean 1994 & $\begin{array}{l}\text { Study was not randomised: "Formal randomization was precluded by instrument availability, but } \\
\text { the time of day did not influence allocation." }\end{array}$ \\
\hline Malik 2007 & Comparison of two open techniques. \\
\hline Malik 2009 & $\begin{array}{l}\text { Statistical analyses included nonrandomised participants: "The patients were divided into group } \\
\text { A (OA) and group B (VAECA) on patient choice and by coin toss, where patients did not opt for any } \\
\text { particular technique." }\end{array}$ \\
\hline Mantoglu 2013 & $\begin{array}{l}\text { Study was only pseudo-randomised: "The patients were randomized into two groups }[\ldots] \text { accord- } \\
\text { ing to admitting day. Patients who were admitted on odd days were treated with LA the others with } \\
\text { OA." }\end{array}$ \\
\hline Marzouk 2002 & $\begin{array}{l}\text { Study was not randomised: "The patients were assigned [to LA or OA] according to insurance com- } \\
\text { pany approval." }\end{array}$ \\
\hline McAnena 1992 & $\begin{array}{l}\text { Study was not randomised: "Formal randomization was precluded on occasions by instrument } \\
\text { availability but not by the time of day at which the operation was performed." }\end{array}$ \\
\hline Milewczyk 1998 & $\begin{array}{l}\text { In a letter from 1999, Dr. Michalik explained that an open list of random codes was used. Thus, the } \\
\text { study was randomised, but without allocation concealment. The } 2003 \text { publication reported on } 200 \\
\text { participants (instead of } 80 \text { as before), but the trial periods overlapped. Therefore, this is one large } \\
\text { study with an interim analysis. }\end{array}$ \\
\hline Moberg 1998 & The study had no control group. \\
\hline Moldovanu 2010 & The study was retrospective. \\
\hline Mugomba 2001 & Choice of surgical procedure was made nonrandomly by the senior surgeon. \\
\hline Naver 1994 & $\begin{array}{l}\text { Choice of surgical procedure depended on the availability of a laparoscopic surgeon: "In the pres- } \\
\text { ence of a surgeon with laparoscopic experience laparoscopy was performed". }\end{array}$ \\
\hline Niebuhr 1992 & Choice of surgical procedure depended on the availability of surgeon and technical equipment. \\
\hline Näf 1996 & The study was retrospective. \\
\hline Oka 2004 & Choice of surgical procedure was "based on the schedule of the attending surgeon on call". \\
\hline Padankatti 2008 & $\begin{array}{l}\text { In the abstract, it was described that "children were allocated randomly". In the main text, howev- } \\
\text { er, it was stated that "cases were allocated into open and laparoscopic groups based on surgeon } \\
\text { preference". }\end{array}$ \\
\hline
\end{tabular}




\begin{tabular}{|c|c|}
\hline Study & Reason for exclusion \\
\hline Planells Roig 1993 & Choice of surgical procedure depended on the surgical team being on call. \\
\hline Prado 1997 & Control group consisted of cases operated on in a different hospital. \\
\hline Pruett 1994 & Study used historical control group. \\
\hline Raakow 1993 & Choice of surgical procedure depended on the availability of surgeon and technical equipment. \\
\hline Rashid 2013 & $\begin{array}{l}\text { In an email dated December 5th, 2014, the first author stated: "We had an open random list hang- } \\
\text { ing on the wall in the surgical consultancy room. This random list was generated by a computer." } \\
\text { Thus, the study was randomised, but without allocation concealment. }\end{array}$ \\
\hline & $\begin{array}{l}\text { In addition, the study investigated interval rather than acute appendectomy. Operations took place } \\
\text { about } 2 \text { months after the initial appendicitis episode which was managed conservatively. }\end{array}$ \\
\hline
\end{tabular}

\begin{tabular}{ll}
\hline Reiertsen 1994 & $\begin{array}{l}\text { The study was non-randomised: "The patients were selected for a conventional or laparoscopic } \\
\text { procedure by the surgeon on call." }\end{array}$ \\
\hline Richards 1993 & $\begin{array}{l}\text { Study used a parallel control group, but "a randomized trial of open versus laparoscopic appen- } \\
\text { dectomy could not be completed in Nashville." }\end{array}$
\end{tabular}

Ritter 1998 Choice of surgical procedure depended on the availability of surgeon and technical equipment.

Rückert 1993 This short abstract described a "prospective randomized" study, in which 51 LA and 150 OA cases were compared. No results were given. Considering the imbalance of the groups, the study seemed not to be randomised. A letter to the first author in November 2001 failed to elicit any answer.

Sarihan 1994 This randomised controlled trial compared peritoneal drainage versus no drainage in perforated
appendicitis.

Sayed Hassen $1996 \quad$ Choice of surgical procedure depended on the availability of surgeon and technical equipment. Assignment depended "on whether the surgeon on call was an 'open' or 'laparoscopic' surgeon for the purpose of the study."

Schramm $1994 \quad$ The study was non-randomised, because choice of surgical technique depended on the availability of a laparoscopic surgeon and severity of symptoms.

Schroder $1993 \quad \begin{aligned} & \text { Study was non-randomised: "The selection of traditional or laparoscopic appendectomy was } \\ & \text { based on the availability of laparoscopic equipment and the surgeon's experience. No patient was } \\ & \text { denied laparoscopic surgery based on severity of disease." }\end{aligned}$

Schäfer 1997 The study used a historical control group.

Sfez $1993 \quad \begin{aligned} & \text { The authors described the study as "prospective". The two groups of } 18 \text { and } 20 \text { cases had compara- } \\ & \text { ble baseline characteristics, but randomisation was not mentioned anywhere in the text. A letter to } \\ & \text { the author remained unanswered. }\end{aligned}$

Shalaby $2001 \quad$ This randomised clinical trial compared three techniques of closing the appendix stump in laparoscopic appendectomy. Conventional appendectomy was not evaluated.

Shen 2012

The assignment to the groups was not randomised. "Some of the insurance companies are approving only OA..., in most cases, the patient was the one to choose the approach after the surgeon explained the procedure...".

Shirazi 2010

Study was only pseudo-randomised: "quasi-experimental study" and "alternatively assigned to group A or B". 


\begin{tabular}{|c|c|}
\hline Study & Reason for exclusion \\
\hline Sosa 1993 & $\begin{array}{l}\text { The study has a retrospective design. The control cases (open appendectomy) were randomly se- } \\
\text { lected from all patients who had undergone open appendectomy. }\end{array}$ \\
\hline St Peter 2010 & The study compared an operative with a conservative approach. \\
\hline Tarnoff 1998 & The study was not randomised. \\
\hline Tate 1993b & $\begin{array}{l}\text { "Patients were not randomized: laparoscopy was performed when a suitably trained surgeon and } \\
\text { laparoscopic instruments were available." }\end{array}$ \\
\hline Thon 1996 & The study was not randomised. \\
\hline Thorell 1999 & The study was not randomised. \\
\hline Till 1994 & $\begin{array}{l}\text { The assignment to conventional or laparoscopic appendectomy was based on the availability of la- } \\
\text { paroscopically experienced surgeons. }\end{array}$ \\
\hline Ure 1992 & The control group consisted only of the converted cases. \\
\hline Van den Broek 2000 & The assignment to groups was made according to severity of symptoms. \\
\hline Varlet 1994 & The study was retrospective. \\
\hline Whitworth 1998 & The study was not randomised. \\
\hline Wullstein 2001 & The study was retrospective. \\
\hline Zaninotto 1995 & $\begin{array}{l}\text { In the } 1994 \text { abstract, the authors claimed that participants were "randomly assigned to open or } \\
\text { laparoscopic surgery". In the full paper, however, the study appeared to be at best pseudo-ran- } \\
\text { domised: "Four surgeons [...] were allocated to laparoscopic treatment (LAP) or open (OPEN) treat- } \\
\text { ment. [...] Each surgeon always performed only one type of operation." }\end{array}$ \\
\hline
\end{tabular}

LA: laparoscopic appendectomy

OA: open appendectomy

VAECA: video-assisted laparoscopic extracorporeal appendectomy

Characteristics of studies awaiting assessment [ordered by study ID]

Esposito 1997

\begin{tabular}{ll}
\hline Methods & Randomised controlled trial \\
& Centres: one (?) (Italy) \\
\hline Participants & $\begin{array}{l}\text { Participants: with proven acute appendicitis } \\
\text { Gender: } 45 \% \text { female } \\
\text { Age: } 17 \text { (mean) for LA and OA } \\
\text { Histology: not reported }\end{array}$ \\
\hline Interventions & LA: not specified ( $\mathrm{n}=45)$ \\
& An: not specified ( $\mathrm{n}=33)$ \\
\hline Outcomes & Hospital stay; duration of surgery; complications \\
\hline Notes & Follow-up: not reported \\
\hline
\end{tabular}


Esposito 1997 (Continued)
No appendectomy: not reported
Declaration of interest: not reported
Funding: not reported
Preoperative imaging: not reported
Analgesia requirements: not reported
Sample size calculation: not reported

Hoff 1995

\begin{tabular}{|c|c|}
\hline Methods & $\begin{array}{l}\text { Randomised controlled trial } \\
\text { Centres: one (?) (Netherlands) }\end{array}$ \\
\hline Participants & $\begin{array}{l}\text { Participants: symptoms with of acute appendicitis } \\
\text { Gender: not reported } \\
\text { Age: } 34 \text { and } 32 \text { years (mean) in LA and OA } \\
\text { Histology: not reported }\end{array}$ \\
\hline Interventions & $\begin{array}{l}\text { LA: not specified }(n=45) \\
\text { OA: not specified }(n=33) \\
\text { Antibiotics: not reported }\end{array}$ \\
\hline Outcomes & Pain; general well-being; any complication; hospital stay \\
\hline Notes & $\begin{array}{l}\text { Follow-up: not reported } \\
\text { No appendectomy: not reported } \\
\text { Declaration of interest: not reported } \\
\text { Funding: not reported } \\
\text { Preoperative imaging: not reported } \\
\text { Analgesia requirements: not reported } \\
\text { Sample size calculation: not reported }\end{array}$ \\
\hline
\end{tabular}

Loh 1992

\begin{tabular}{ll}
\hline Methods & Randomised controlled trial \\
& Centres: one (?) (UK) \\
\hline Participants & Participants: not specified \\
& Gender: not reported \\
& Age: not reported \\
& Histology: not reported \\
\hline Interventions & LA: not specified ( $n=25 ?)$ \\
& OA: not specified ( $n=25 ?)$ \\
Antibiotics: not reported
\end{tabular}


Loh 1992 (Continued)

Outcomes Hospital stay; analgesic consumption; morbidity; pain; return to work; wound infection

\begin{tabular}{|c|c|}
\hline \multirow[t]{6}{*}{ Notes } & $\begin{array}{l}\text { Follow-up: } 3 \text { weeks } \\
\text { No appendectomy: not reported }\end{array}$ \\
\hline & Declaration of interest: not reported \\
\hline & Funding: not reported \\
\hline & Preoperative imaging: not reported \\
\hline & Analgesia requirements: not reported \\
\hline & Sample size calculation: not reported \\
\hline
\end{tabular}

Rohr 1994

\begin{tabular}{|c|c|}
\hline Methods & $\begin{array}{l}\text { Randomised controlled trial } \\
\text { Centres: one (?) (France) }\end{array}$ \\
\hline Participants & $\begin{array}{l}\text { Participants: clinical characteristics of acute appendicitis } \\
\text { Gender: } 0 \% \text { female } \\
\text { Age: } 28 \text { and } 31 \text { years (mean) in LA and OA } \\
\text { Histology: } 85 \% \text { inflamed }\end{array}$ \\
\hline Interventions & $\begin{array}{l}\text { LA: by using three trocras }(n=30) \\
\text { OA: McBurney's incision }(n=30) \\
\text { Antibiotics: not reported }\end{array}$ \\
\hline Outcomes & $\begin{array}{l}\text { Hospital stay; time of surgery; analgesic consumption; morbidity; pain; return to work and sports; } \\
\text { wound infection }\end{array}$ \\
\hline Notes & $\begin{array}{l}\text { Follow-up: } 4 \text { months } \\
\text { No appendectomy: not reported } \\
\text { Declaration of interest: not reported } \\
\text { Funding: not reported } \\
\text { Preoperative imaging: not reported } \\
\text { Analgesia requirements: not reported } \\
\text { Sample size calculation: not reported }\end{array}$ \\
\hline
\end{tabular}

Talha 2015

\begin{tabular}{ll}
\hline Methods & Randomised controlled trial \\
& Centres: one (?) (Egypt) \\
\hline Participants & Participants: preoperative diagnosis of complicated appendicitis \\
& Gender: not reported \\
Age: not reported \\
Histology: not reported \\
\hline
\end{tabular}


Talha 2015 (Continued)

\begin{tabular}{ll} 
Interventions & $\begin{array}{l}\text { LA: not specified }(n=56) \\
\text { OA: not specified }(n=50) \\
\text { Antibiotics: not reported }\end{array}$ \\
\hline Outcomes & $\begin{array}{l}\text { Septic complications; time of surgery; time until return of liquid diet; hospital stay; pain; quality of } \\
\text { life score; wound infections }\end{array}$ \\
\hline Fotes & $\begin{array}{l}\text { Follow-up: not reported } \\
\text { No appendectomy: not reported }\end{array}$ \\
& Declaration of interest: not reported \\
Funding: not reported & Preoperative imaging: not reported \\
Analgesia requirements: less use of analgesics after LA & Sample size calculation: not reported
\end{tabular}

LA: laparoscopic appendectomy

OA: open appendectomy

Characteristics of ongoing studies [ordered by study ID]

\section{ChicTR-TRC-14005067}

\begin{tabular}{ll}
\hline Trial name or title & $\begin{array}{l}\text { Impact of children laparoscopic appendectomy on gastrointestinal function: a randomized con- } \\
\text { trolled trial }\end{array}$ \\
\hline Methods & Randomised parallel control \\
\hline Participants & $\begin{array}{l}\text { 1. the diagnosis of appendicitis in children; } \\
\text { 2. older than } 3 \text { years old, less than } 16 \text { years old; } \\
\text { 3. informed consent. }\end{array}$ \\
\hline
\end{tabular}

Interventions $\quad$ LA $(n=60)$ versus OA $(n=60)$

\begin{tabular}{ll}
\hline Outcomes & Incidence of residual pus of peritoneal fluid; \\
& wound infection; \\
& incidence of abdominal abscess; \\
& occurrence of incisional hernia; \\
& recovery of gastrointestinal function; \\
& incidence of postoperative fever; \\
& incidence of postoperative ileus. \\
\hline Starting date & 2014/08/01 \\
\hline Contact information & Name: Zhong Wenyi \\
E-Mail: dbzhongwy@163.com & Address: Department of Minimally Invasive Surgery,
\end{tabular}


ChicTR-TRC-14005067 (Continued)

Da-bu People hospital; Meizhou City,

Guangdong Province, China

\begin{tabular}{ll}
\hline Notes & Main ID: ChiCTR-TRC-14005067 \\
& Primary sponsor: Department of Minimally Invasive Surgery, Da-bu People hospital, Meizhou City, \\
& Guangdong \\
Source of funding: by raised
\end{tabular}

\section{IRCT2015102724747N1}

\begin{tabular}{|c|c|}
\hline Trial name or title & $\begin{array}{l}\text { Laparoscopic versus open appendectomy: compare outcomes and complications in the treatment } \\
\text { of complicated appendicitis }\end{array}$ \\
\hline Methods & Randomised controlled trial \\
\hline Participants & All patients suspected of having complicated acute appendicitis \\
\hline Interventions & LA versus $O A(n=96)$ \\
\hline \multirow[t]{2}{*}{ Outcomes } & Primary outcome: duration of surgery \\
\hline & $\begin{array}{l}\text { Secondary outcome: surgical site infection; intra-abdominal abscess; pain, complications such as } \\
\text { nosocomial infections, hernia incisional; length of hospitalisation; need for further surgery }\end{array}$ \\
\hline Starting date & $2015 / 11 / 22$ (no longer recruiting) \\
\hline \multirow[t]{3}{*}{ Contact information } & Name: Amir Mangouri \\
\hline & E-Mail: amir.mangouri@gmail.com \\
\hline & Adress: Azadi Ave, Hafez Square, Sina hospital Tabriz, Iran \\
\hline \multirow[t]{3}{*}{ Notes } & Main ID: http://en.irct.ir/trial/20823 \\
\hline & Primary sponsor: Vice Chancellor for research, Tabriz University of Medical Sciences \\
\hline & Source of funding: Vice Chancellor for research, Tabriz University of Medical Sciences \\
\hline
\end{tabular}

\section{IRCT201703088375N12}

\begin{tabular}{ll}
\hline Trial name or title & $\begin{array}{l}\text { Comparison of complications and outcomes of three methods of laparoscopic appendectomy: sin- } \\
\text { gle incision and open appendectomy in paediatric patients }\end{array}$ \\
\hline Methods & Randomised controlled trial \\
\hline Participants & In paediatric age group (from 8 years old to 14 years old) \\
\hline Interventions & The interventions consisted of: \\
& - group 1 to undergo open conventional appendectomy, \\
& - group 2 to be treated by laparoscopic appendectomy, \\
& group 3 appendectomy to be done using single incision appendectomy. \\
\hline
\end{tabular}


IRCT201703088375N12 (Continued)

Outcomes Duration of surgery; wound infection; size of the scar; hospital stay time

\begin{tabular}{ll}
\hline Starting date & 2017/08/07 (recruitment completed) \\
\hline Contact information & Name: Dr Amir Kazem Vejdan \\
& E-Mail: vejdan_sa@bums.ac.ir \\
& Address: Birjand University of Medical Sciences, Imam Reza hospital, Birjand, Iran \\
\hline Notes & Main ID: http://en.irct.ir/trial/8854 \\
& Primary sponsor: Birjand University of Medical Sciences \\
& Source of funding: Birjand University of Medical Sciences
\end{tabular}

\section{NCT01260064}

\begin{tabular}{|c|c|}
\hline Trial name or title & $\begin{array}{l}\text { Open appendectomy versus laparoscopic appendectomy - the outcomes of various appendectomy } \\
\text { techniques in a high-volume centre }\end{array}$ \\
\hline Methods & Randomised controlled trial \\
\hline Participants & Patients with a preoperative diagnosis of acute appendicitis \\
\hline Interventions & $\begin{array}{l}\text { LA (appendiceal stump secured by metal endoclips; } n=50 \text { ) versus LA (appendiceal stump secured } \\
\text { by intracorporeal suture ligation; } n=50 \text { ) versus } O A(n=50)\end{array}$ \\
\hline \multirow[t]{2}{*}{ Outcomes } & $\begin{array}{l}\text { Primary outcome: quality of life after various appendectomy procedures to be measured by a spe- } \\
\text { cific quality-of-life index (after } 1 \text { month) }\end{array}$ \\
\hline & Secondary outcome: cost-effectiveness \\
\hline Starting date & 2010/10/01 \\
\hline \multirow[t]{4}{*}{ Contact information } & Name: Halil Alis \\
\hline & E-Mail: not reported \\
\hline & Address: Dr. Sadi Konuk Training and Research Hospital, Istanbul, \\
\hline & Turkey, 34147 \\
\hline \multirow[t]{3}{*}{ Notes } & Main ID: NCT01260064 \\
\hline & Primary sponsor: Bakirkoy Dr. Sadi Konuk Research and Training Hospital \\
\hline & Source of funding: Bakirkoy Dr. Sadi Konuk Research and Training Hospital \\
\hline
\end{tabular}

UMIN000003711

\begin{tabular}{ll} 
Trial name or title & Value of laparoscopic appendectomy in perforated appendicitis - a randomised trial \\
\hline Methods & Parallel randomised
\end{tabular}


UMIN000003711 (Continued)
Participants
Patients diagnosed as having complicated appendicitis with peritonitis or abscess formation, by abdominal examination, laboratory data, or CT

\section{Interventions}

$\operatorname{LA}(n=50)$ versus $O A(n=50)$
Primary outcome: occurrence of postoperative infectious complications

Secondary outcomes: rate of reoperation; indices of postoperative recovery (analgesic use, oral intake restart, physical activity, bowel movement); duration of hospital stay; operating time

\begin{tabular}{ll}
\hline Starting date & 2008/10/01 (no longer recruiting) \\
\hline Contact information & Name: Shunichiro Komatsu \\
& E-Mail: skomat@nagoya2.jrc.or.jp \\
& Address: 2-9, Myokencho, Showaku, Nagoya City, Aichi Pref. \\
& Japan \\
\hline Notes & Main ID: JPRN-UMIN000003711 \\
& Primary sponsor: Nagoya Daini Red Cross Hospital \\
& Source of funding: Nagoya Daini Red Cross Hospital
\end{tabular}

CT:computed tomography

LA: laparoscopic appendectomy

OA: open appendectomy

\section{DATA AND ANALYSES}

\section{Comparison 1. Laparoscopic versus open appendectomy in adults or adolescents}

\begin{tabular}{|c|c|c|c|c|}
\hline Outcome or subgroup title & No. of studies & $\begin{array}{l}\text { No. of partici- } \\
\text { pants }\end{array}$ & Statistical method & Effect size \\
\hline 1 Pain intensity on day 1 & 20 & 2421 & $\begin{array}{l}\text { Mean Difference (IV, Random, 95\% } \\
\mathrm{CI})\end{array}$ & $-0.75[-1.04,-0.45]$ \\
\hline $\begin{array}{l}\text { 1.1 Studies reporting mean } \\
\text { data }\end{array}$ & 20 & 2421 & $\begin{array}{l}\text { Mean Difference (IV, Random, 95\% } \\
\mathrm{CI})\end{array}$ & $-0.75[-1.04,-0.45]$ \\
\hline 2 Wound infections & 63 & 7612 & $\begin{array}{l}\text { Peto Odds Ratio (Peto, Fixed, 95\% } \\
\mathrm{Cl} \text { ) }\end{array}$ & $0.42[0.35,0.51]$ \\
\hline 3 Intra-abdominal abscesses & 53 & 6677 & $\begin{array}{l}\text { Peto Odds Ratio (Peto, Fixed, 95\% } \\
\mathrm{Cl} \text { ) }\end{array}$ & $1.65[1.12,2.43]$ \\
\hline 4 Length of hospital stay & 46 & 5127 & $\begin{array}{l}\text { Mean Difference (IV, Random, 95\% } \\
\mathrm{Cl} \text { ) }\end{array}$ & $-0.96[-1.23,-0.70]$ \\
\hline $\begin{array}{l}4.1 \text { Studies reporting out- } \\
\text { come }\end{array}$ & 46 & 5127 & $\begin{array}{l}\text { Mean Difference (IV, Random, 95\% } \\
\mathrm{CI} \text { ) }\end{array}$ & $-0.96[-1.23,-0.70]$ \\
\hline
\end{tabular}




\begin{tabular}{lllll}
\hline Outcome or subgroup title & No. of studies & $\begin{array}{l}\text { No. of partici- } \\
\text { pants }\end{array}$ & Statistical method & Effect size \\
\hline $\begin{array}{l}5 \text { Time until return to normal } \\
\text { activity }\end{array}$ & 17 & 1653 & $\begin{array}{l}\text { Mean Difference (IV, Random, 95\% } \\
\text { Cl) }\end{array}$ & -4.97 [-6.77, -3.16] \\
\hline $\begin{array}{l}5.1 \text { Studies reporting out- } \\
\text { come }\end{array}$ & 17 & 1653 & $\begin{array}{l}\text { Mean Difference (IV, Random, 95\% } \\
\text { Cl) }\end{array}$ & -4.97 [-6.77, -3.16] \\
\hline
\end{tabular}

Analysis 1.1. Comparison 1 Laparoscopic versus open appendectomy in adults or adolescents, Outcome 1 Pain intensity on day 1.

\begin{tabular}{|c|c|c|c|c|c|c|c|c|}
\hline \multirow[t]{2}{*}{ Study or subgroup } & \multicolumn{2}{|c|}{ Laparoscopic } & \multicolumn{2}{|c|}{ Open } & \multirow{2}{*}{\multicolumn{2}{|c|}{$\begin{array}{l}\text { Mean Difference } \\
\text { Random, } 95 \% \mathrm{Cl}\end{array}$}} & \multirow[t]{2}{*}{ Weight } & \multirow{2}{*}{$\begin{array}{l}\text { Mean Difference } \\
\text { Random, } 95 \% \mathrm{CI}\end{array}$} \\
\hline & $\mathbf{N}$ & Mean(SD) & $\mathbf{N}$ & Mean(SD) & & & & \\
\hline \multicolumn{9}{|c|}{ 1.1.1 Studies reporting mean data } \\
\hline Cipe 2014 & 126 & $3.1(1.2)$ & 120 & $3.4(1.3)$ & + & & $7.93 \%$ & $-0.33[-0.64,-0.02]$ \\
\hline Eichen 1994 & 97 & $5.2(1.5)$ & 96 & $5.9(1.3)$ & + & & $7.5 \%$ & $-0.69[-1.09,-0.29]$ \\
\hline Gundavda 2012 & 30 & $3.7(3.7)$ & 30 & $4.3(4.3)$ & & & $1.71 \%$ & $-0.53[-2.56,1.5]$ \\
\hline Hebebrand 1994 & 34 & $2.9(1.5)$ & 23 & $4.2(2.5)$ & $\longrightarrow$ & & $3.79 \%$ & $-1.28[-2.42,-0.14]$ \\
\hline Ignacio 2003 & 26 & $3.5(2.1)$ & 26 & $4(1.9)$ & 1 & - & $3.99 \%$ & $-0.5[-1.59,0.59]$ \\
\hline Kaplan 2009 & 40 & $2.8(0.6)$ & 43 & $3.3(0.6)$ & + & & $8.15 \%$ & $-0.55[-0.81,-0.29]$ \\
\hline Kargar 2011 & 50 & $1.8(1.7)$ & 50 & $1.4(1.3)$ & & $\longrightarrow$ & $6.49 \%$ & $0.42[-0.16,1]$ \\
\hline Kazemier 1997 & 97 & $3.5(2.4)$ & 104 & $5.9(2.5)$ & $\longrightarrow$ & & $5.99 \%$ & $-2.34[-3.01,-1.67]$ \\
\hline Minné 1997 & 27 & $3.7(3.7)$ & 23 & $4(4)$ & 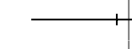 & & $1.55 \%$ & $-0.3[-2.45,1.85]$ \\
\hline Mutter 1996 & 50 & $4.7(2)$ & 50 & $4.4(1.7)$ & & + & $5.78 \%$ & $0.3[-0.41,1.01]$ \\
\hline Reiertsen 1997 & 42 & $5(2)$ & 42 & $5.4(1.4)$ & $\longrightarrow$ & - & $5.64 \%$ & $-0.4[-1.14,0.34]$ \\
\hline Ricca 2007 & 22 & $3.5(2.1)$ & 22 & $4(1.9)$ & & - & $3.65 \%$ & $-0.49[-1.67,0.69]$ \\
\hline Schippers 1997 & 20 & $4.2(2.3)$ & 20 & $4.8(2.2)$ & $\longrightarrow$ & - & $2.93 \%$ & $-0.61[-2.01,0.79]$ \\
\hline Singh 2017 & 15 & $3(3)$ & 15 & $4.5(4.5)$ & & & $1.02 \%$ & $-1.53[-4.28,1.22]$ \\
\hline Sozutek 2013 & 25 & $2(1)$ & 25 & $3.5(1)$ & $\rightarrow$ & & $6.66 \%$ & $-1.5[-2.05,-0.95]$ \\
\hline Tate 1993a & 70 & $4.7(2.1)$ & 70 & $5.3(2.3)$ & $\longrightarrow$ & & $5.68 \%$ & $-0.6[-1.33,0.13]$ \\
\hline Vallribera 2003 & 34 & $2.9(1.4)$ & 28 & $4.3(1.4)$ & $\multimap$ & & $5.82 \%$ & $-1.38[-2.08,-0.68]$ \\
\hline Witten 1998 & 95 & $5.2(5.2)$ & 100 & $5.8(5.8)$ & 1 & & $2.58 \%$ & $-0.6[-2.14,0.94]$ \\
\hline 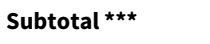 & 1233 & & 1188 & & $\diamond$ & & $100 \%$ & $-0.75[-1.04,-0.45]$ \\
\hline \multicolumn{9}{|c|}{ Heterogeneity: $\mathrm{Tau}^{2}=0.26 ; \mathrm{Chi}^{2}=71.86, \mathrm{df}=19(\mathrm{P}<0.0001) ; \mathrm{I}^{2}=73.56 \%$} \\
\hline \multicolumn{9}{|c|}{ Test for overall effect: $Z=4.95(P<0.0001)$} \\
\hline Total $* \star \star$ & 1233 & & 1188 & & $\diamond$ & & $100 \%$ & $-0.75[-1.04,-0.45]$ \\
\hline \multicolumn{9}{|c|}{ Test for overall effect: $Z=4.95(P<0.0001)$} \\
\hline & & & Favou & roscopic & -2.5 & 2.5 & Favour & \\
\hline
\end{tabular}


Analysis 1.2. Comparison 1 Laparoscopic versus open appendectomy in adults or adolescents, Outcome 2 Wound infections.

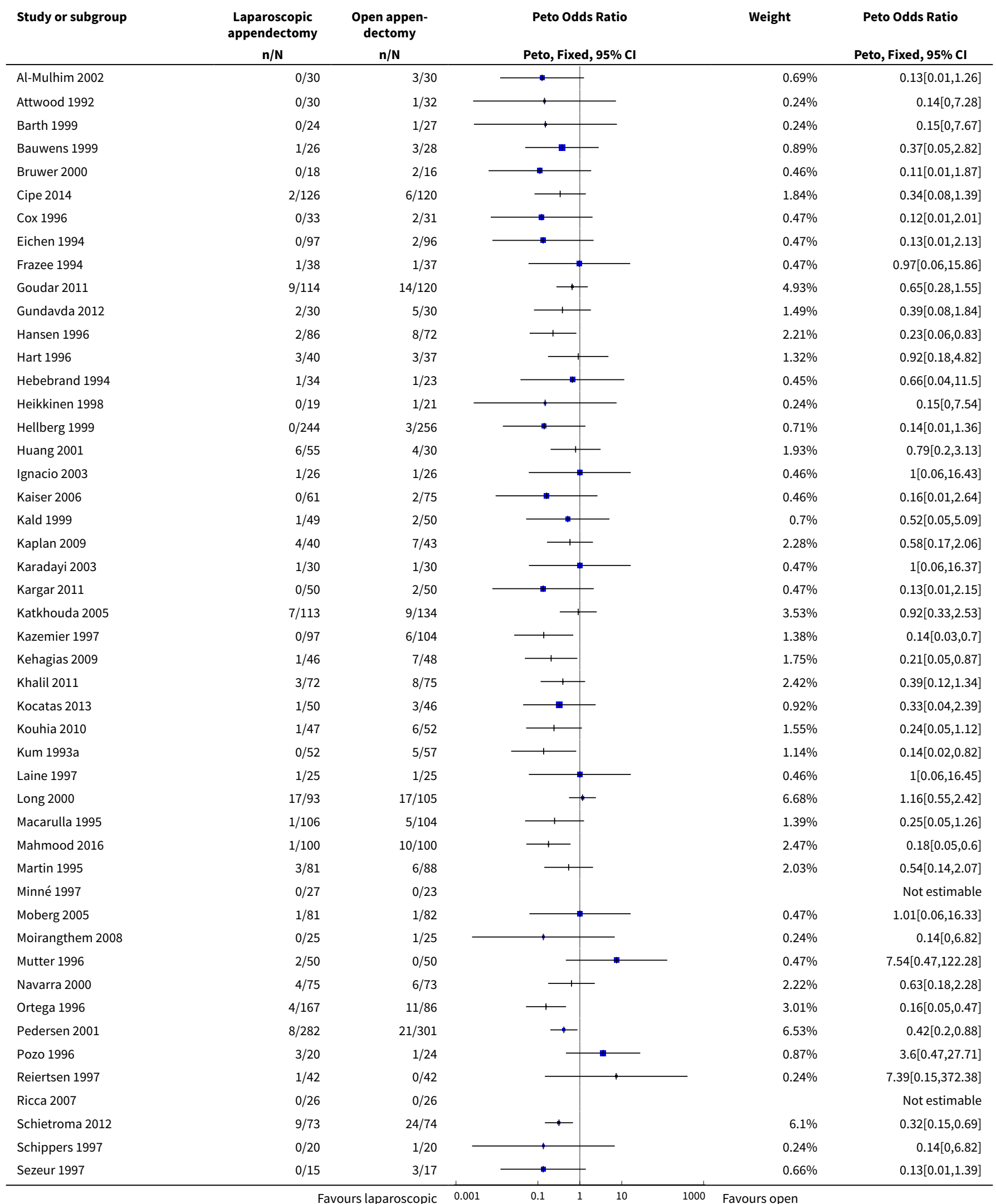




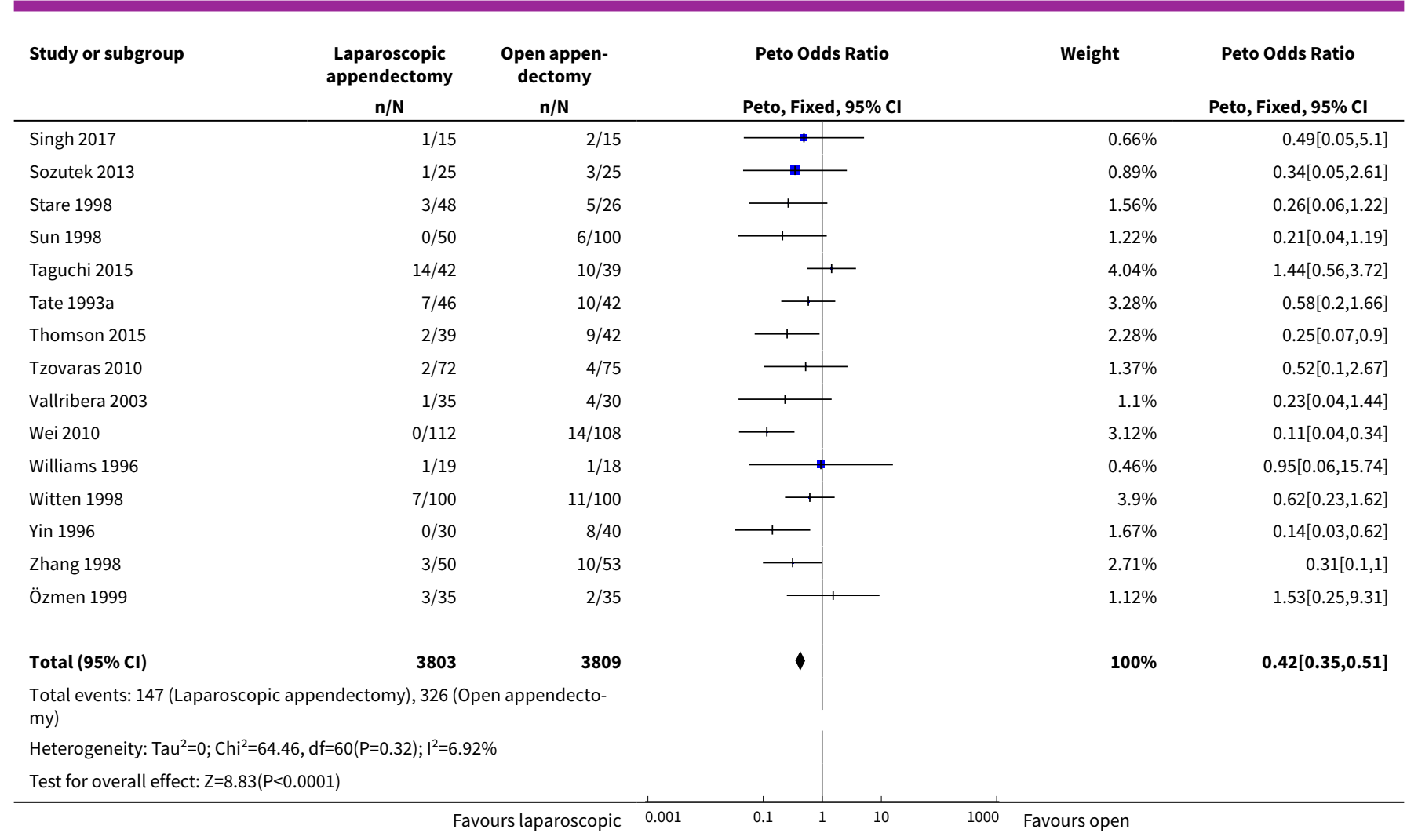

Analysis 1.3. Comparison 1 Laparoscopic versus open appendectomy in adults or adolescents, Outcome 3 Intra-abdominal abscesses.

\begin{tabular}{|c|c|c|c|c|c|}
\hline Study or subgroup & $\begin{array}{c}\text { Laparoscopic } \\
n / N\end{array}$ & $\begin{array}{c}\text { Open } \\
\mathrm{n} / \mathrm{N}\end{array}$ & $\begin{array}{c}\text { Peto Odds Ratio } \\
\text { Peto, Fixed, } 95 \% \mathrm{Cl}\end{array}$ & Weight & $\begin{array}{c}\text { Peto Odds Ratio } \\
\text { Peto, Fixed, } 95 \% \mathrm{Cl}\end{array}$ \\
\hline Al-Mulhim 2002 & $0 / 30$ & $0 / 30$ & & & Not estimable \\
\hline Attwood 1992 & $0 / 30$ & $0 / 32$ & & & Not estimable \\
\hline Bauwens 1999 & $0 / 26$ & $0 / 28$ & & & Not estimable \\
\hline Bruwer 2000 & $1 / 18$ & $0 / 16$ & - & $0.98 \%$ & $6.61[0.13,335.5]$ \\
\hline Cipe 2014 & $6 / 126$ & $4 / 120$ & $\longrightarrow$ & $9.42 \%$ & $1.44[0.41,5.09]$ \\
\hline Cox 1996 & $0 / 33$ & $0 / 31$ & & & Not estimable \\
\hline Eichen 1994 & $0 / 97$ & $1 / 96$ & - & $0.98 \%$ & $0.13[0,6.75]$ \\
\hline Frazee 1994 & $1 / 38$ & $0 / 37$ & I & $0.98 \%$ & $7.2[0.14,362.84]$ \\
\hline Goudar 2011 & $0 / 114$ & $0 / 120$ & & & Not estimable \\
\hline Hansen 1996 & $0 / 86$ & $0 / 72$ & & & Not estimable \\
\hline Hart 1996 & $3 / 40$ & $0 / 37$ & & $2.85 \%$ & $7.22[0.73,71.69]$ \\
\hline Hebebrand 1994 & $0 / 34$ & $0 / 23$ & & & Not estimable \\
\hline Heikkinen 1998 & $0 / 19$ & $0 / 21$ & & & Not estimable \\
\hline Hellberg 1999 & $5 / 244$ & $3 / 256$ & $\longrightarrow$ & $7.71 \%$ & $1.74[0.43,7.04]$ \\
\hline Helmy 2001 & $0 / 50$ & $0 / 50$ & & & Not estimable \\
\hline Huang 2001 & $0 / 55$ & $0 / 30$ & & & Not estimable \\
\hline Ignacio 2003 & $0 / 26$ & $0 / 26$ & & & Not estimable \\
\hline Kaiser 2006 & $0 / 61$ & $0 / 75$ & & & Not estimable \\
\hline Kald 1999 & $1 / 49$ & $0 / 50$ & $\longrightarrow$ & $0.98 \%$ & $7.54[0.15,380.14]$ \\
\hline Karadayi 2003 & $0 / 30$ & $0 / 30$ & & & Not estimable \\
\hline Katkhouda 2005 & $6 / 113$ & $4 / 134$ & $\longrightarrow$ & $9.36 \%$ & $1.81[0.51,6.45]$ \\
\hline
\end{tabular}




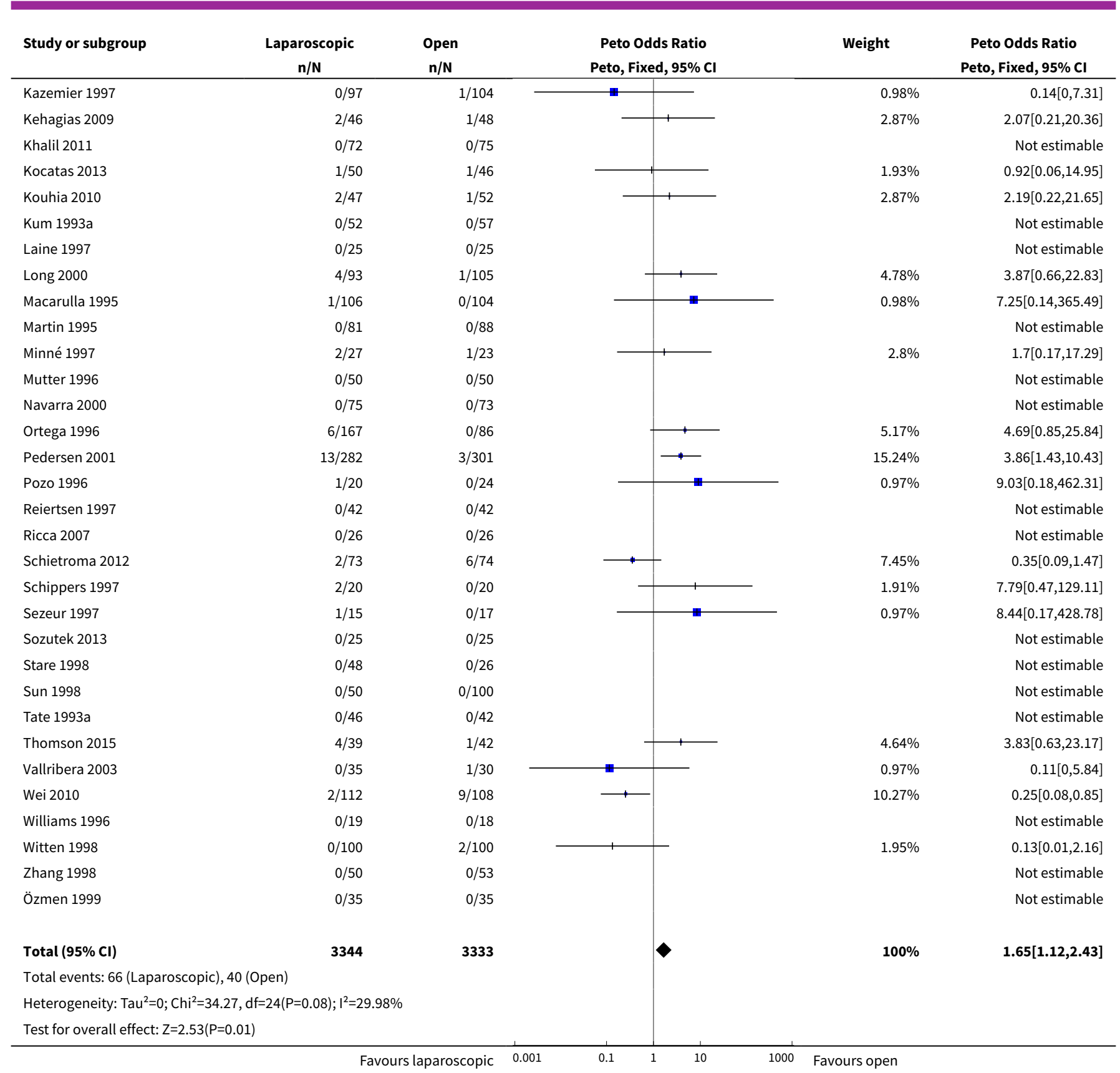

Analysis 1.4. Comparison 1 Laparoscopic versus open appendectomy in adults or adolescents, Outcome 4 Length of hospital stay.

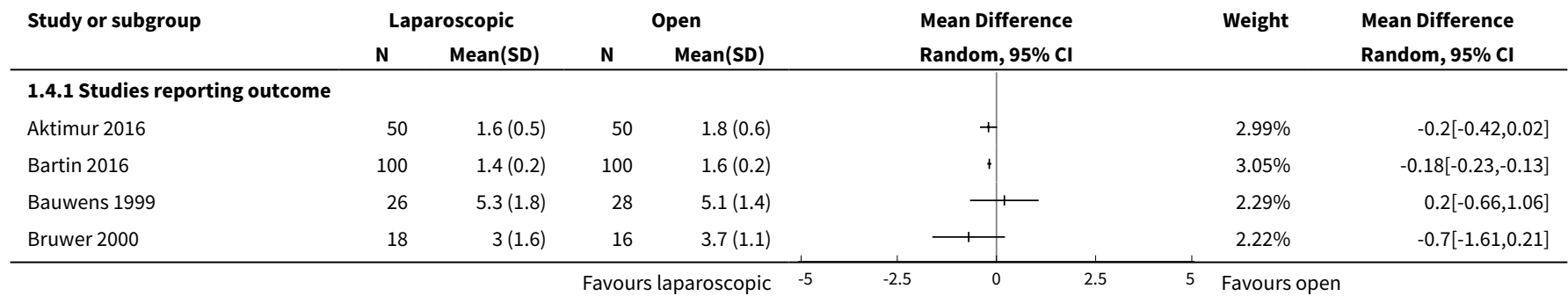




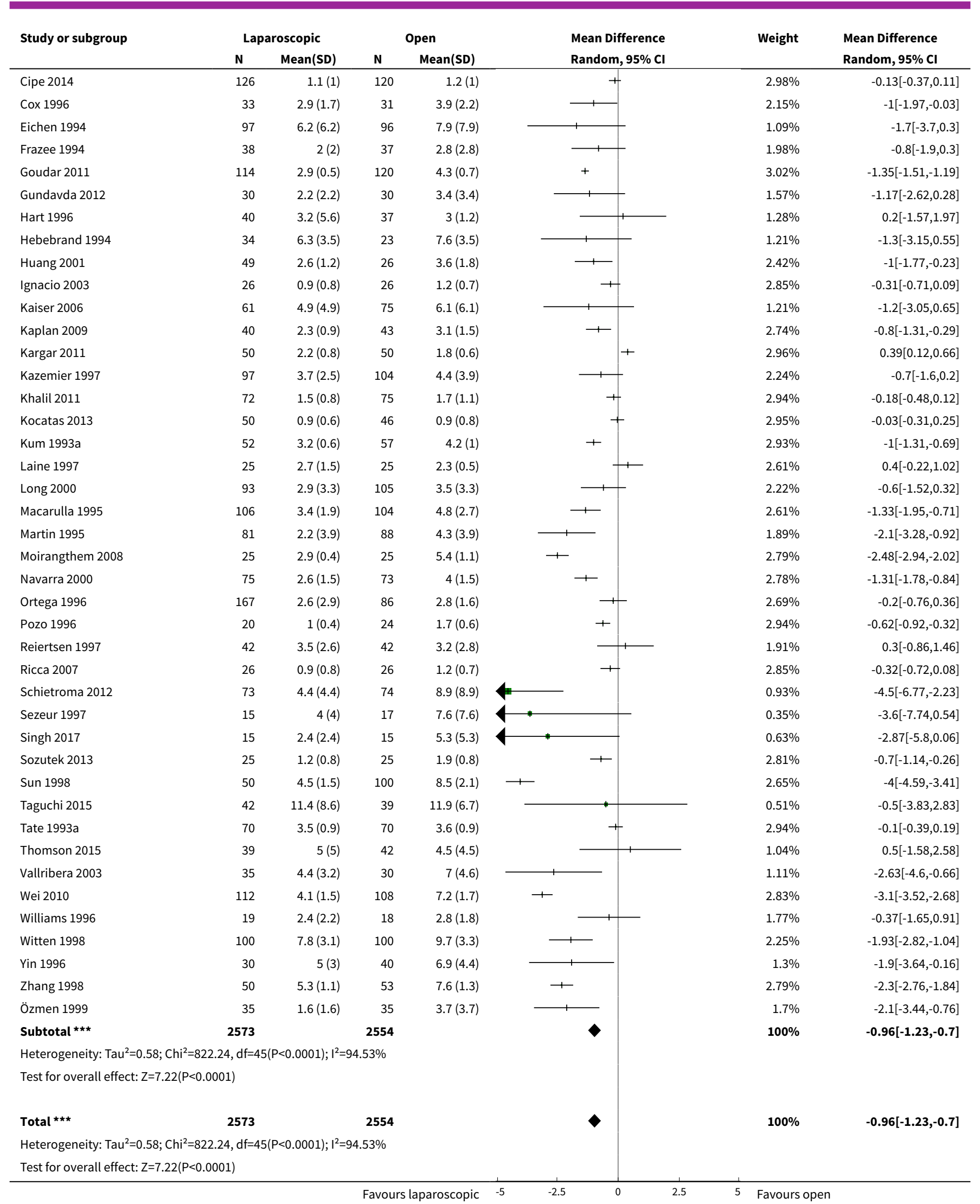


Analysis 1.5. Comparison 1 Laparoscopic versus open appendectomy in adults or adolescents, Outcome 5 Time until return to normal activity.

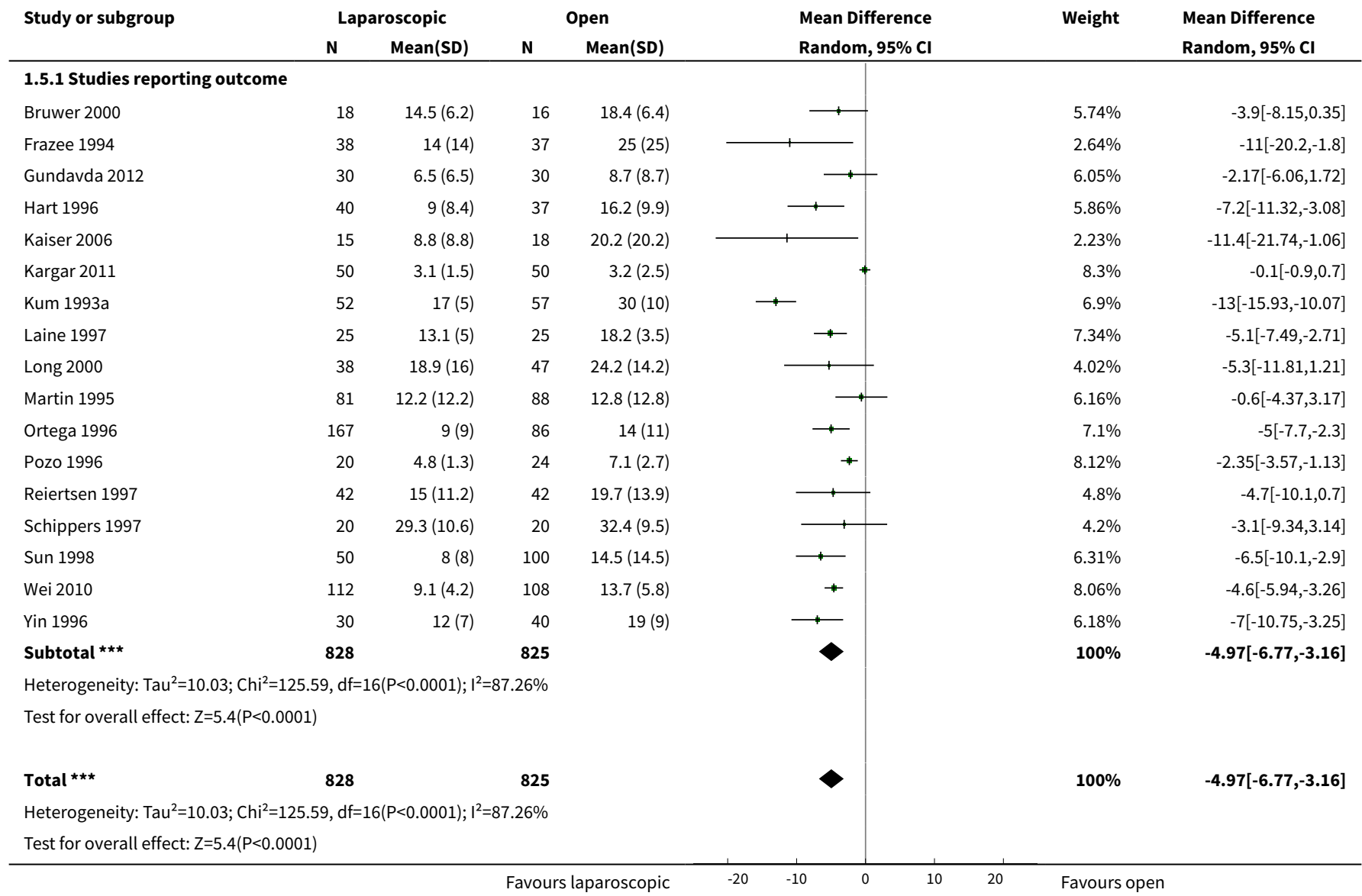

\section{Comparison 2. Laparoscopic versus open appendectomy in children}

\begin{tabular}{lllll}
\hline Outcome or subgroup title & No. of studies & $\begin{array}{l}\text { No. of partici- } \\
\text { pants }\end{array}$ & Statistical method & Effect size \\
\hline $\begin{array}{l}1 \text { Pain intensity on day } 1 \text { (cm } \\
\text { VAS) }\end{array}$ & 1 & 61 & $\begin{array}{l}\text { Mean Difference (IV, Random, 95\% } \\
\text { Cl) }\end{array}$ & $-0.8[-1.65,0.05]$ \\
\hline $\begin{array}{l}1.1 \text { Studies reporting out- } \\
\text { come }\end{array}$ & 1 & 61 & $\begin{array}{l}\text { Mean Difference (IV, Random, 95\% } \\
\text { Cl) }\end{array}$ & $-0.8[-1.65,0.05]$ \\
\hline $\begin{array}{l}\text { W Wound infections } \\
\text { 3 Intra-abdominal abscesses }\end{array}$ & 9 & 1245 & $\begin{array}{l}\text { Peto Odds Ratio (Peto, Fixed, 95\% } \\
\text { Cl) }\end{array}$ & $0.25[0.15,0.42]$ \\
\hline $\begin{array}{l}4 \text { Length of hospital stay } \\
\text { 4.1 Studies reporting out- }\end{array}$ & 6 & 1185 & $\begin{array}{l}\text { Peto Odds Ratio (Peto, Fixed, 95\% } \\
\text { Cl) }\end{array}$ & $0.54[0.24,1.22]$ \\
\hline come & 6 & 316 & Mean Difference (IV, Fixed, 95\% CI) & $-0.81[-1.01,-0.62]$ \\
\hline
\end{tabular}




\begin{tabular}{lllll}
\hline Outcome or subgroup title & No. of studies & $\begin{array}{l}\text { No. of partici- } \\
\text { pants }\end{array}$ & Statistical method & Effect size \\
\hline $\begin{array}{l}5 \text { Time until return to normal } \\
\text { activity }\end{array}$ & 1 & 383 & $\begin{array}{l}\text { Mean Difference (IV, Random, 95\% } \\
\text { Cl) }\end{array}$ & $-0.5[-1.30,0.30]$ \\
\hline $\begin{array}{l}5.1 \text { Studies reporting out- } \\
\text { come }\end{array}$ & 1 & 383 & $\begin{array}{l}\text { Mean Difference (IV, Random, 95\% } \\
\text { Cl) }\end{array}$ & $-0.5[-1.30,0.30]$ \\
\hline
\end{tabular}

Analysis 2.1. Comparison 2 Laparoscopic versus open appendectomy in children, Outcome 1 Pain intensity on day 1 (cm VAS).

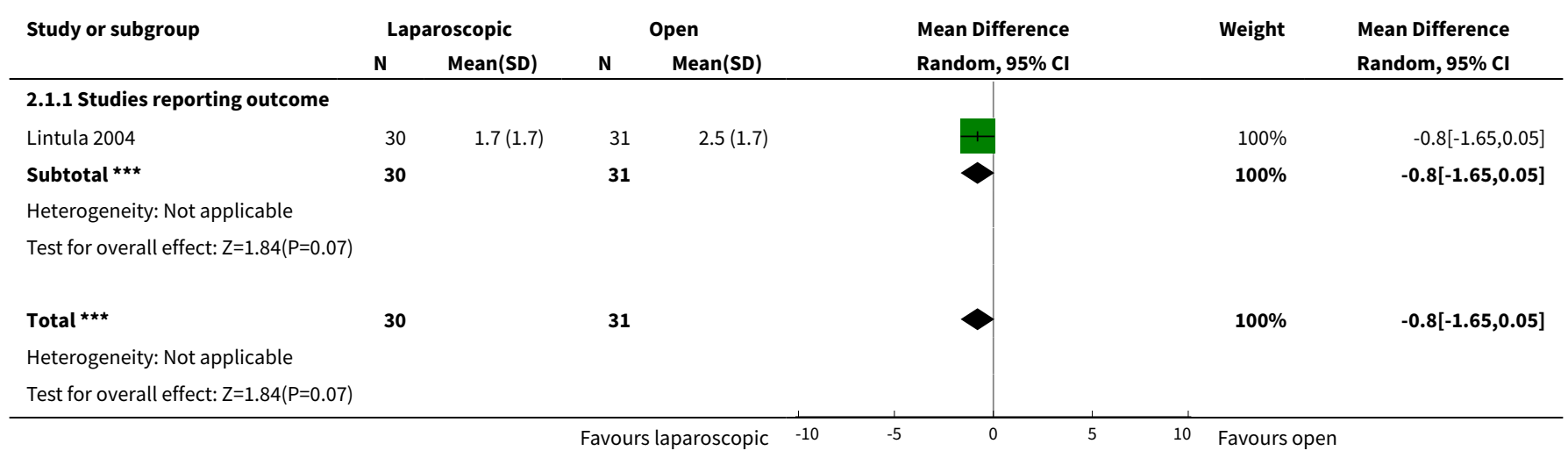

Analysis 2.2. Comparison 2 Laparoscopic versus open appendectomy in children, Outcome 2 Wound infections.

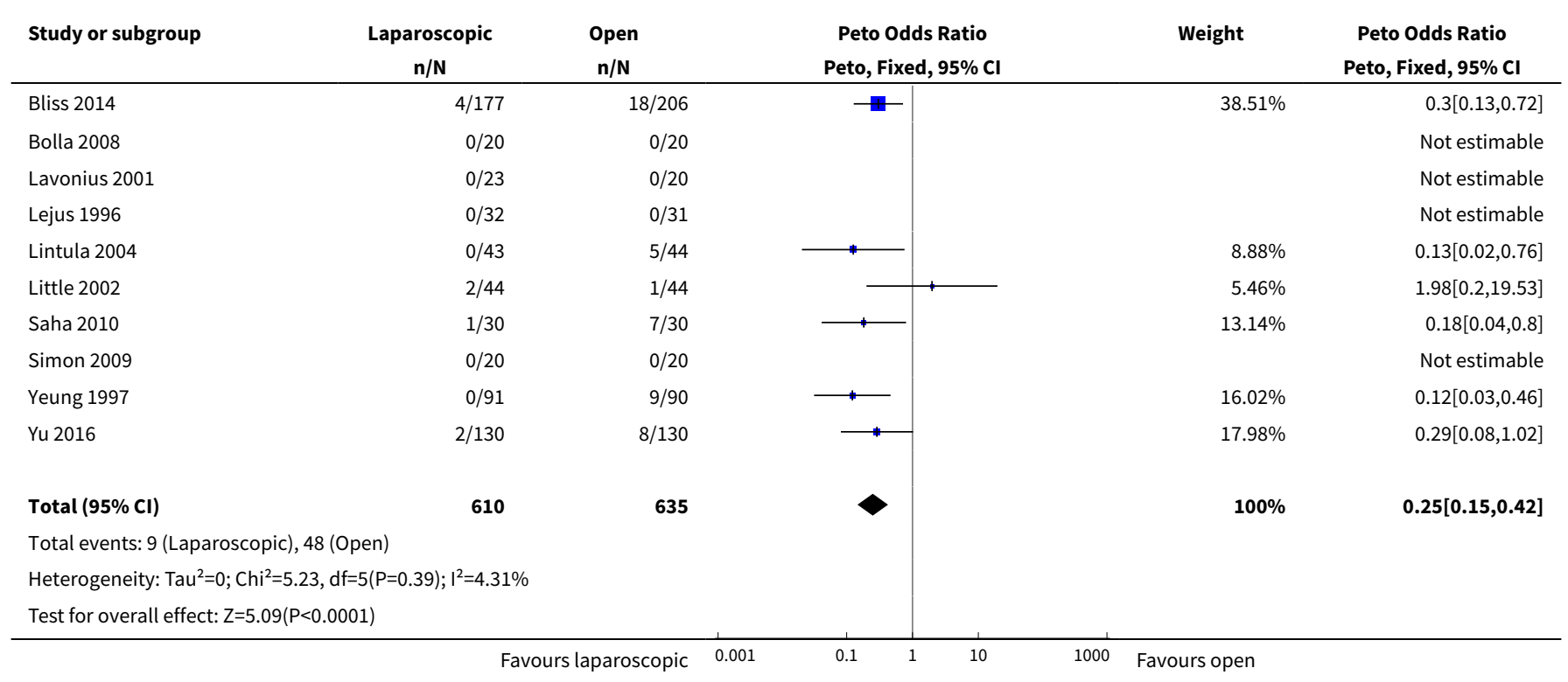


Analysis 2.3. Comparison 2 Laparoscopic versus open appendectomy in children, Outcome 3 Intra-abdominal abscesses.

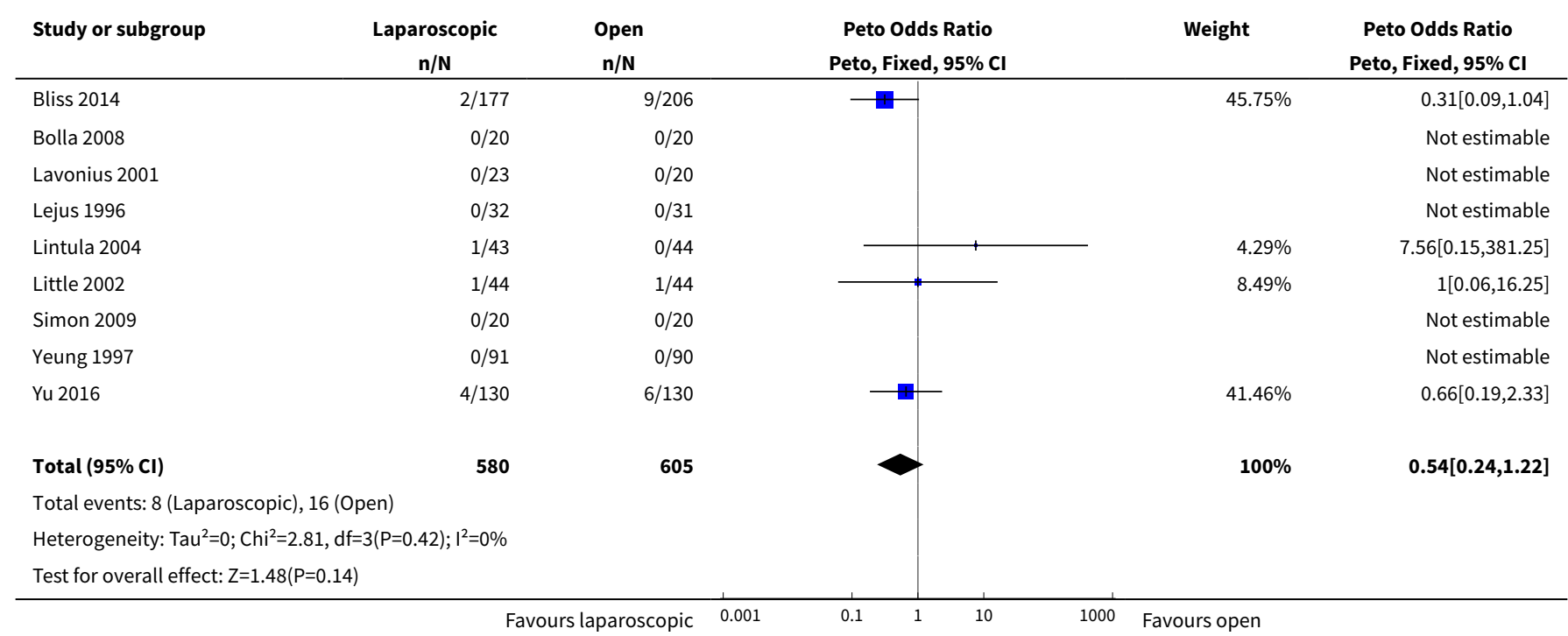

Analysis 2.4. Comparison 2 Laparoscopic versus open appendectomy in children, Outcome 4 Length of hospital stay.

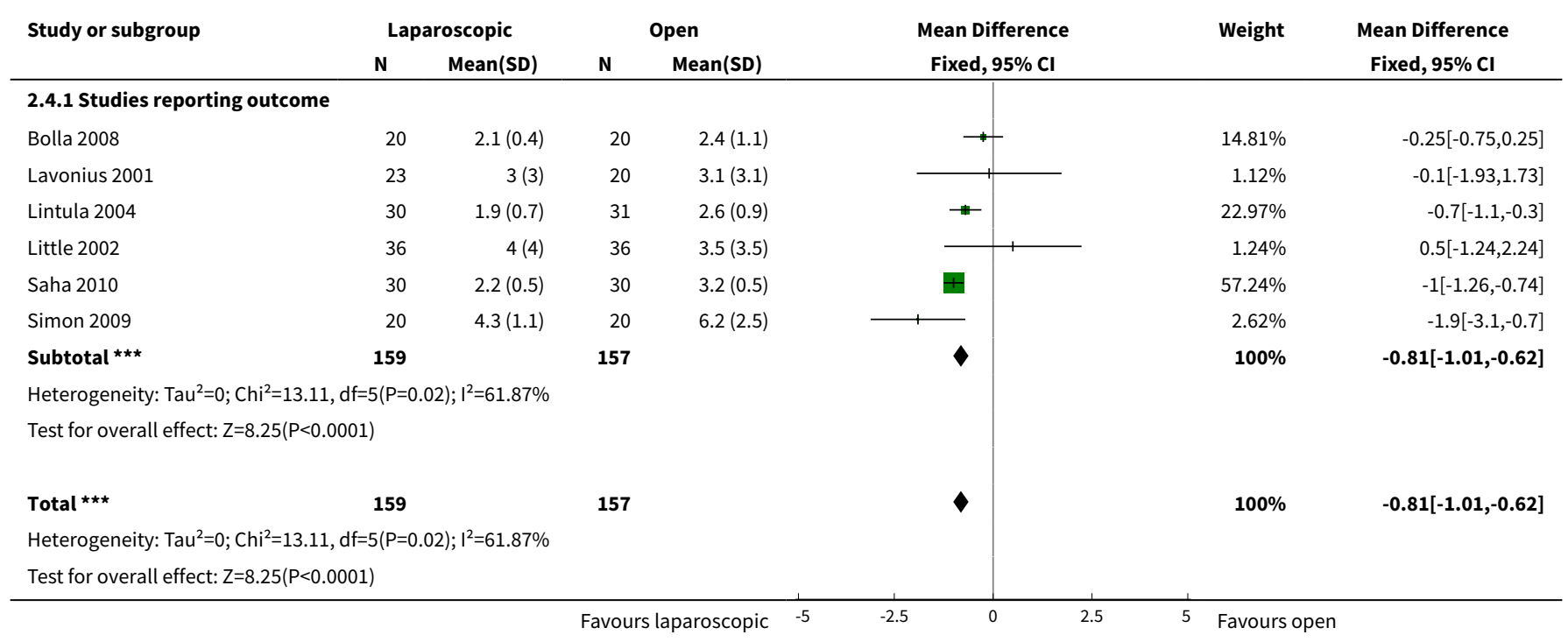

Analysis 2.5. Comparison 2 Laparoscopic versus open appendectomy in children, Outcome 5 Time until return to normal activity.

\begin{tabular}{|c|c|c|c|c|c|c|c|c|}
\hline \multirow[t]{2}{*}{ Study or subgroup } & \multicolumn{2}{|c|}{ Laparoscopic } & \multicolumn{2}{|c|}{ Open } & \multirow{2}{*}{\multicolumn{2}{|c|}{$\begin{array}{l}\text { Mean Difference } \\
\text { Random, } 95 \% \mathrm{Cl}\end{array}$}} & \multirow[t]{2}{*}{ Weight } & \multirow{2}{*}{$\begin{array}{l}\text { Mean Difference } \\
\text { Random, } 95 \% \mathrm{Cl}\end{array}$} \\
\hline & $\mathbf{N}$ & Mean(SD) & $\mathbf{N}$ & Mean(SD) & & & & \\
\hline \multicolumn{9}{|c|}{ 2.5.1 Studies reporting outcome } \\
\hline Bliss 2014 & 177 & $7.1(4)$ & 206 & $7.6(4)$ & & & $100 \%$ & $-0.5[-1.3,0.3]$ \\
\hline
\end{tabular}




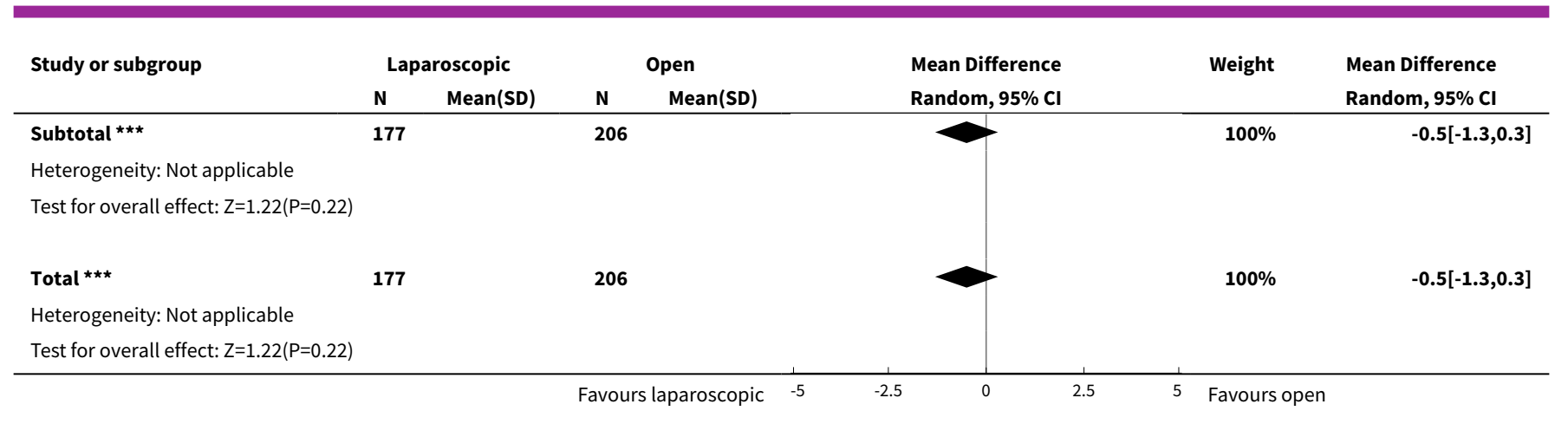

Comparison 3. Subgroup analysis (publication date) - laparoscopic versus open appendectomy in adults or adolescents

\begin{tabular}{|c|c|c|c|c|}
\hline Outcome or subgroup title & No. of studies & $\begin{array}{l}\text { No. of partici- } \\
\text { pants }\end{array}$ & Statistical method & Effect size \\
\hline 1 Pain intensity on day 1 & 7 & 613 & $\begin{array}{l}\text { Mean Difference (IV, Random, 95\% } \\
\mathrm{CI})\end{array}$ & $-0.52[-0.98,-0.06]$ \\
\hline $\begin{array}{l}\text { 1.1 Studies reporting mean } \\
\text { data }\end{array}$ & 7 & 613 & $\begin{array}{l}\text { Mean Difference (IV, Random, 95\% } \\
\mathrm{CI})\end{array}$ & $-0.52[-0.98,-0.06]$ \\
\hline 2 Wound infections & 19 & 2217 & $\begin{array}{l}\text { Peto Odds Ratio (Peto, Fixed, 95\% } \\
\mathrm{Cl} \text { ) }\end{array}$ & $0.37[0.27,0.51]$ \\
\hline $\begin{array}{l}3 \text { Intra-abdominal abscess- } \\
\text { es }\end{array}$ & 11 & 1466 & $\begin{array}{l}\text { Peto Odds Ratio (Peto, Fixed, 95\% } \\
\mathrm{Cl} \text { ) }\end{array}$ & $0.82[0.44,1.51]$ \\
\hline 4 Length of hospital stay & 17 & 1977 & $\begin{array}{l}\text { Mean Difference (IV, Random, 95\% } \\
\mathrm{CI})\end{array}$ & $-0.84[-1.23,-0.44]$ \\
\hline $\begin{array}{l}\text { 4.1 Studies reporting out- } \\
\text { come }\end{array}$ & 17 & 1977 & $\begin{array}{l}\text { Mean Difference (IV, Random, 95\% } \\
\mathrm{Cl} \text { ) }\end{array}$ & $-0.84[-1.23,-0.44]$ \\
\hline $\begin{array}{l}5 \text { Time until return to nor- } \\
\text { mal activity }\end{array}$ & 3 & 380 & $\begin{array}{l}\text { Mean Difference (IV, Random, 95\% } \\
\mathrm{Cl} \text { ) }\end{array}$ & $-2.27[-5.81,1.26]$ \\
\hline $\begin{array}{l}5.1 \text { Studies reporting out- } \\
\text { come }\end{array}$ & 3 & 380 & $\begin{array}{l}\text { Mean Difference (IV, Random, 95\% } \\
\mathrm{Cl} \text { ) }\end{array}$ & $-2.27[-5.81,1.26]$ \\
\hline
\end{tabular}

\section{Analysis 3.1. Comparison 3 Subgroup analysis (publication date) - laparoscopic versus open appendectomy in adults or adolescents, Outcome 1 Pain intensity on day 1.}

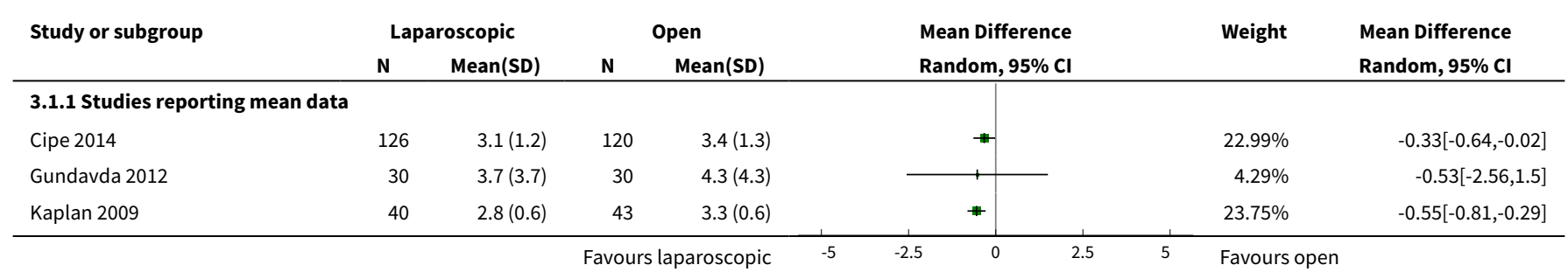




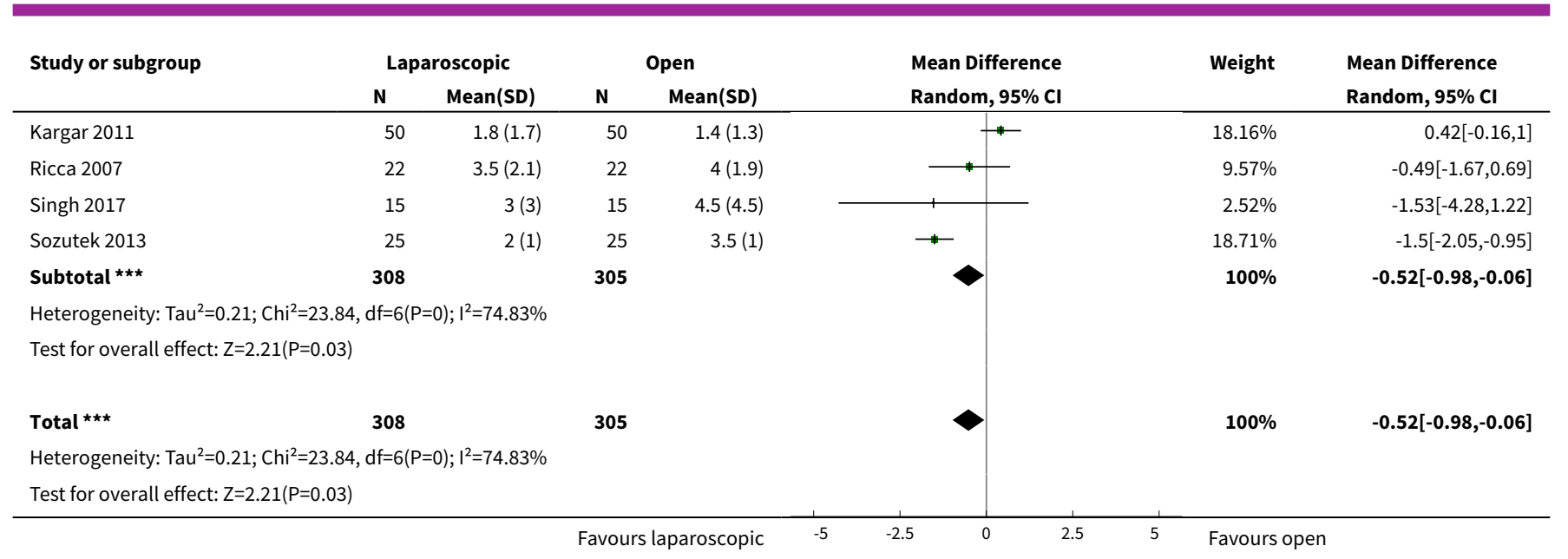

Analysis 3.2. Comparison 3 Subgroup analysis (publication date) - laparoscopic versus open appendectomy in adults or adolescents, Outcome 2 Wound infections.

\begin{tabular}{|c|c|c|c|c|c|}
\hline \multirow[t]{2}{*}{ Study or subgroup } & \multirow{2}{*}{$\begin{array}{c}\text { Favours la- } \\
\text { paroscopic } \\
\mathbf{n} / \mathbf{N}\end{array}$} & \multirow{2}{*}{$\begin{array}{l}\text { Open } \\
n / N \\
\end{array}$} & Peto Odds Ratio & \multirow[t]{2}{*}{ Weight } & \multirow{2}{*}{$\begin{array}{c}\text { Peto Odds Ratio } \\
\text { Peto, Fixed, } 95 \% \mathrm{Cl}\end{array}$} \\
\hline & & & Peto, Fixed, 95\% Cl & & \\
\hline Cipe 2014 & $2 / 126$ & $6 / 120$ & $\longrightarrow$ & $4.74 \%$ & $0.34[0.08,1.39]$ \\
\hline Goudar 2011 & $9 / 114$ & $14 / 120$ & $\rightarrow$ & $12.69 \%$ & $0.65[0.28,1.55]$ \\
\hline Gundavda 2012 & $2 / 30$ & $5 / 30$ & \begin{tabular}{l|l}
1 & \\
\end{tabular} & $3.84 \%$ & $0.39[0.08,1.84]$ \\
\hline Kaplan 2009 & $4 / 40$ & $7 / 43$ & $\longrightarrow$ & $5.88 \%$ & $0.58[0.17,2.06]$ \\
\hline Kehagias 2009 & $1 / 46$ & $7 / 48$ & + & $4.51 \%$ & $0.21[0.05,0.87]$ \\
\hline Khalil 2011 & $3 / 72$ & $8 / 75$ & $\rightarrow$ & $6.25 \%$ & $0.39[0.12,1.34]$ \\
\hline Kocatas 2013 & $1 / 50$ & $3 / 46$ & \begin{tabular}{l|l}
$\hookrightarrow$ &
\end{tabular} & $2.36 \%$ & $0.33[0.04,2.39]$ \\
\hline Kouhia 2010 & $1 / 47$ & $6 / 52$ & 1 & $4 \%$ & $0.24[0.05,1.12]$ \\
\hline Mahmood 2016 & $1 / 100$ & $10 / 100$ & $\longrightarrow$ & $6.37 \%$ & $0.18[0.05,0.6]$ \\
\hline Moirangthem 2008 & $0 / 25$ & $1 / 25$ & & $0.61 \%$ & $0.14[0,6.82]$ \\
\hline Schietroma 2012 & $9 / 73$ & $24 / 74$ & $\longrightarrow$ & $15.71 \%$ & $0.32[0.15,0.69]$ \\
\hline Singh 2017 & $1 / 15$ & $2 / 15$ & & $1.7 \%$ & $0.49[0.05,5.1]$ \\
\hline Sozutek 2013 & $1 / 25$ & $3 / 25$ & - & $2.29 \%$ & $0.34[0.05,2.61]$ \\
\hline Taguchi 2015 & $14 / 42$ & $10 / 39$ & 1 & $10.42 \%$ & $1.44[0.56,3.72]$ \\
\hline Thomson 2015 & $2 / 39$ & $9 / 42$ & 1 & $5.86 \%$ & $0.25[0.07,0.9]$ \\
\hline Tzovaras 2010 & $2 / 72$ & $4 / 75$ & \begin{tabular}{l|l}
1 & \\
\end{tabular} & $3.53 \%$ & $0.52[0.1,2.67]$ \\
\hline Wei 2010 & $0 / 112$ & $14 / 108$ & $\longrightarrow$ & $8.03 \%$ & $0.11[0.04,0.34]$ \\
\hline Total $(95 \% \mathrm{CI})$ & 1104 & 1113 & $\diamond$ & $100 \%$ & $0.37[0.27,0.51]$ \\
\hline \multicolumn{6}{|c|}{ Total events: 53 (Favours laparoscopic), 135 (Open) } \\
\hline \multicolumn{6}{|c|}{ Heterogeneity: $\operatorname{Tau}^{2}=0 ; \mathrm{Chi}^{2}=18.44, \mathrm{df}=17(\mathrm{P}=0.36) ; \mathrm{I}^{2}=7.79 \%$} \\
\hline Test for overall effec & & & & & \\
\hline
\end{tabular}


Analysis 3.3. Comparison 3 Subgroup analysis (publication date) - laparoscopic versus open appendectomy in adults or adolescents, Outcome 3 Intra-abdominal abscesses.

\begin{tabular}{|c|c|c|c|c|c|}
\hline Study or subgroup & $\begin{array}{c}\text { Laparoscopic } \\
n / N\end{array}$ & $\begin{array}{c}\text { Open } \\
\mathrm{n} / \mathrm{N}\end{array}$ & $\begin{array}{c}\text { Peto Odds Ratio } \\
\text { Peto, Fixed, } 95 \% \mathrm{Cl}\end{array}$ & Weight & $\begin{array}{c}\text { Peto Odds Ratio } \\
\text { Peto, Fixed, } 95 \% \mathrm{Cl}\end{array}$ \\
\hline Cipe 2014 & $6 / 126$ & $4 / 120$ & - & $23.88 \%$ & $1.44[0.41,5.09]$ \\
\hline Goudar 2011 & $0 / 114$ & $0 / 120$ & & & Not estimable \\
\hline Kehagias 2009 & $2 / 46$ & $1 / 48$ & I & $7.28 \%$ & $2.07[0.21,20.36]$ \\
\hline Khalil 2011 & $0 / 72$ & $0 / 75$ & & & Not estimable \\
\hline Kocatas 2013 & $1 / 50$ & $1 / 46$ & & $4.9 \%$ & $0.92[0.06,14.95]$ \\
\hline Ricca 2007 & $0 / 26$ & $0 / 26$ & & & Not estimable \\
\hline Schietroma 2012 & $2 / 73$ & $6 / 74$ & $\longrightarrow$ & $18.89 \%$ & $0.35[0.09,1.47]$ \\
\hline Sozutek 2013 & $0 / 25$ & $0 / 25$ & & & Not estimable \\
\hline Thomson 2015 & $4 / 39$ & $1 / 42$ & 4 & $11.76 \%$ & $3.83[0.63,23.17]$ \\
\hline Wei 2010 & $2 / 112$ & $9 / 108$ & $\longrightarrow$ & $26.03 \%$ & $0.25[0.08,0.85]$ \\
\hline \multicolumn{6}{|c|}{ Total events: 19 (Laparoscopic), 23 (Open) } \\
\hline \multicolumn{6}{|c|}{ Heterogeneity: $\mathrm{Tau}^{2}=0 ; \mathrm{Chi}^{2}=9.87, \mathrm{df}=6(\mathrm{P}=0.13) ; \mathrm{I}^{2}=39.23 \%$} \\
\hline \multicolumn{6}{|c|}{ Test for overall effect: $\mathrm{Z}=0.64(\mathrm{P}=0.52)$} \\
\hline
\end{tabular}

\section{Analysis 3.4. Comparison 3 Subgroup analysis (publication date) - laparoscopic versus open appendectomy in adults or adolescents, Outcome 4 Length of hospital stay.}

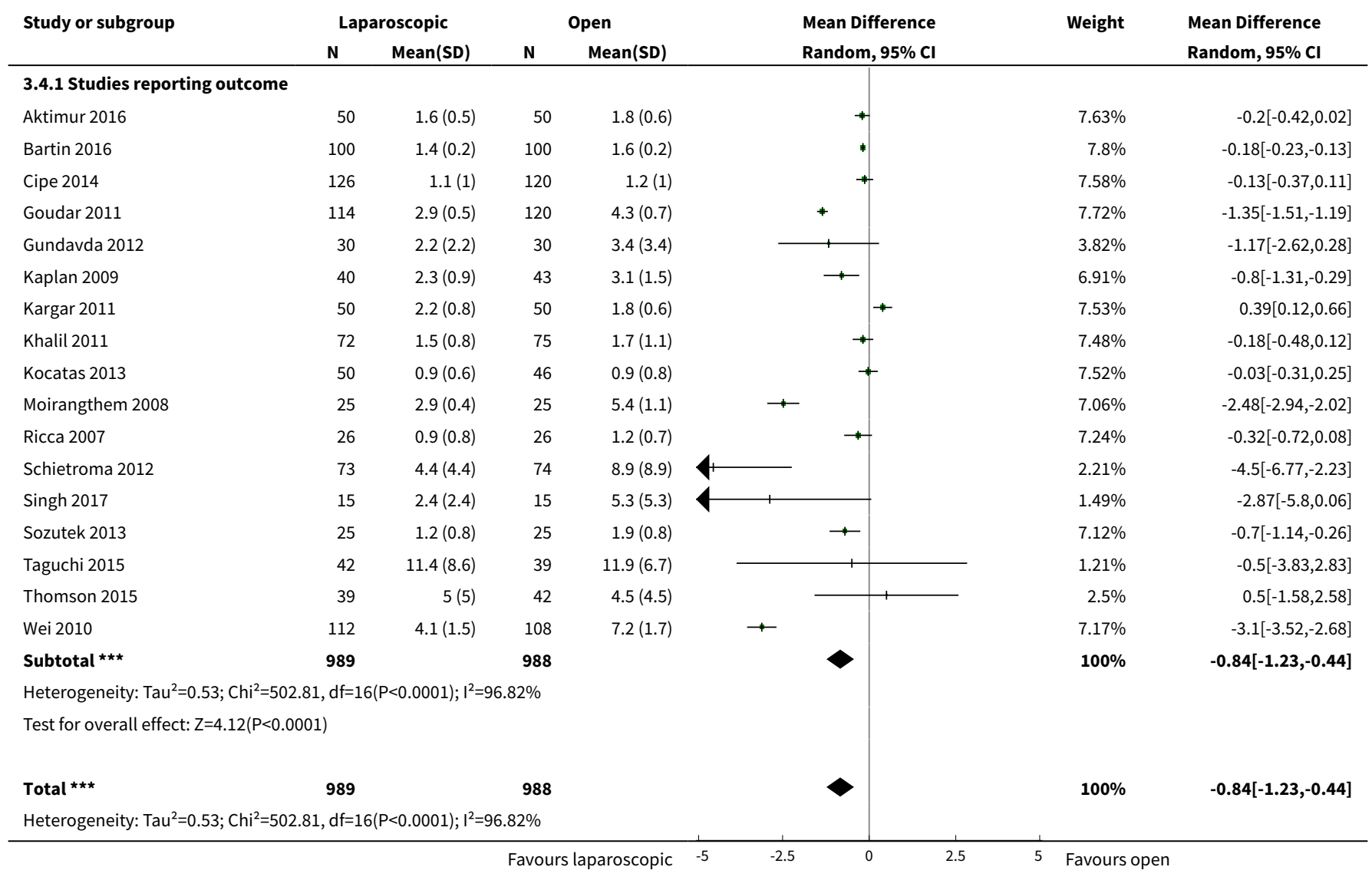




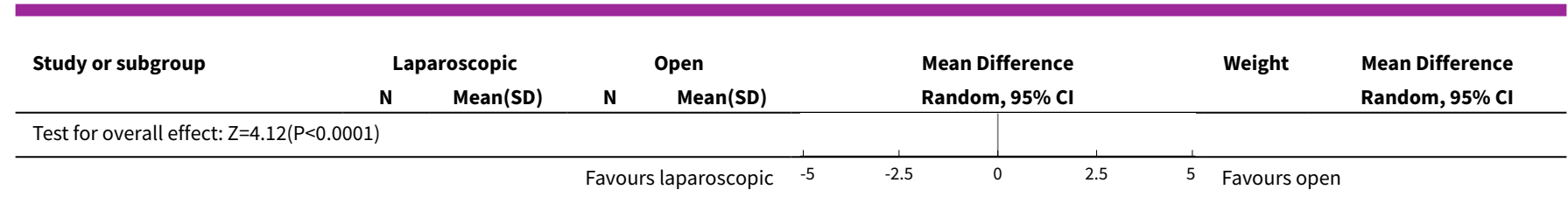

\section{Analysis 3.5. Comparison 3 Subgroup analysis (publication date) - laparoscopic versus open appendectomy in adults or adolescents, Outcome 5 Time until return to normal activity.}

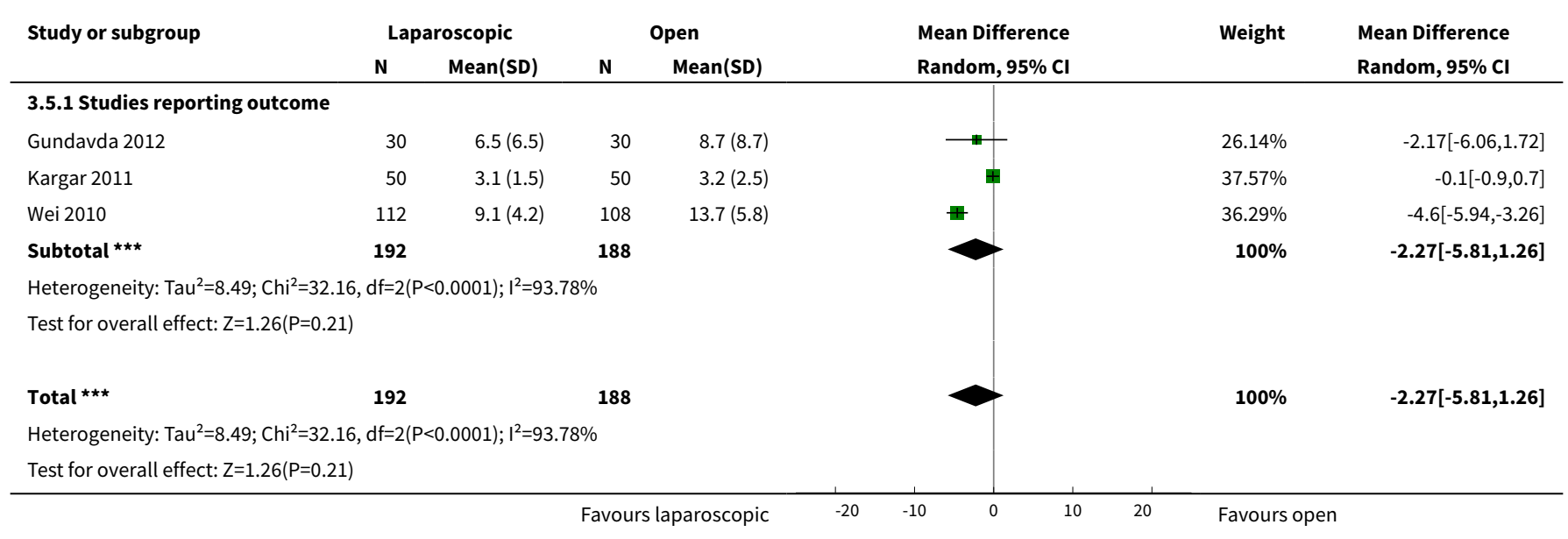

Comparison 4. Subgroup analysis (publication date) - Laparoscopic versus open appendectomy in children

\begin{tabular}{|c|c|c|c|c|}
\hline Outcome or subgroup title & No. of studies & $\begin{array}{l}\text { No. of partici- } \\
\text { pants }\end{array}$ & Statistical method & Effect size \\
\hline 1 Wound infections & 5 & 783 & $\begin{array}{l}\text { Peto Odds Ratio (Peto, Fixed, 95\% } \\
\mathrm{Cl} \text { ) }\end{array}$ & $0.27[0.14,0.52]$ \\
\hline 2 Intra-abdominal abscesses & 4 & 723 & $\begin{array}{l}\text { Peto Odds Ratio (Peto, Fixed, 95\% } \\
\mathrm{Cl} \text { ) }\end{array}$ & $0.45[0.19,1.07]$ \\
\hline $\begin{array}{l}3 \text { Time until return to normal } \\
\text { activity }\end{array}$ & 1 & 383 & $\begin{array}{l}\text { Mean Difference (IV, Random, 95\% } \\
\mathrm{CI})\end{array}$ & $-0.5[-1.30,0.30]$ \\
\hline $\begin{array}{l}\text { 3.1 Studies reporting out- } \\
\text { come }\end{array}$ & 1 & 383 & $\begin{array}{l}\text { Mean Difference (IV, Random, 95\% } \\
\mathrm{CI})\end{array}$ & $-0.5[-1.30,0.30]$ \\
\hline 4 Length of hospital stay & 3 & 140 & Mean Difference (IV, Fixed, 95\% CI) & $-0.88[-1.11,-0.66]$ \\
\hline $\begin{array}{l}4.1 \text { Studies reporting out- } \\
\text { come }\end{array}$ & 3 & 140 & Mean Difference (IV, Fixed, 95\% CI) & $-0.88[-1.11,-0.66]$ \\
\hline
\end{tabular}


Analysis 4.1. Comparison 4 Subgroup analysis (publication date) - Laparoscopic versus open appendectomy in children, Outcome 1 Wound infections.

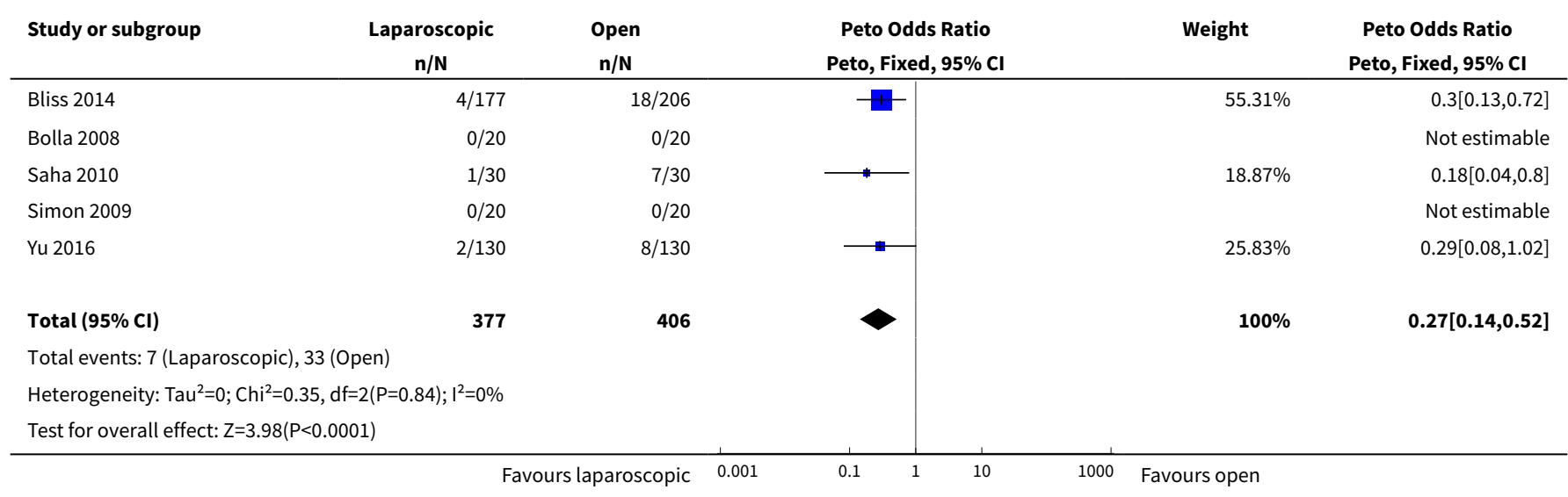

Analysis 4.2. Comparison 4 Subgroup analysis (publication date) - Laparoscopic versus open appendectomy in children, Outcome 2 Intra-abdominal abscesses.

\begin{tabular}{|c|c|c|c|c|c|}
\hline Study or subgroup & $\begin{array}{c}\text { Laparoscopic } \\
n / N\end{array}$ & $\begin{array}{c}\text { Open } \\
\mathrm{n} / \mathrm{N}\end{array}$ & $\begin{array}{c}\text { Peto Odds Ratio } \\
\text { Peto, Fixed, } 95 \% \mathrm{Cl}\end{array}$ & Weight & $\begin{array}{c}\text { Peto Odds Ratio } \\
\text { Peto, Fixed, } 95 \% \mathrm{Cl}\end{array}$ \\
\hline Bliss 2014 & $2 / 177$ & $9 / 206$ & - & $52.46 \%$ & $0.31[0.09,1.04]$ \\
\hline Bolla 2008 & $0 / 20$ & $0 / 20$ & & & Not estimable \\
\hline Simon 2009 & $0 / 20$ & $0 / 20$ & & & Not estimable \\
\hline Yu 2016 & $4 / 130$ & $6 / 130$ & प- & $47.54 \%$ & $0.66[0.19,2.33]$ \\
\hline \multicolumn{6}{|c|}{ Total events: 6 (Laparoscopic), 15 (Open) } \\
\hline \multicolumn{6}{|c|}{ Heterogeneity: $\operatorname{Tau}^{2}=0 ; \mathrm{Chi}^{2}=0.7, \mathrm{df}=1(\mathrm{P}=0.4) ; \mathrm{I}^{2}=0 \%$} \\
\hline Test for overall effect & & & & & \\
\hline
\end{tabular}

Analysis 4.3. Comparison 4 Subgroup analysis (publication date) - Laparoscopic versus open appendectomy in children, Outcome 3 Time until return to normal activity.

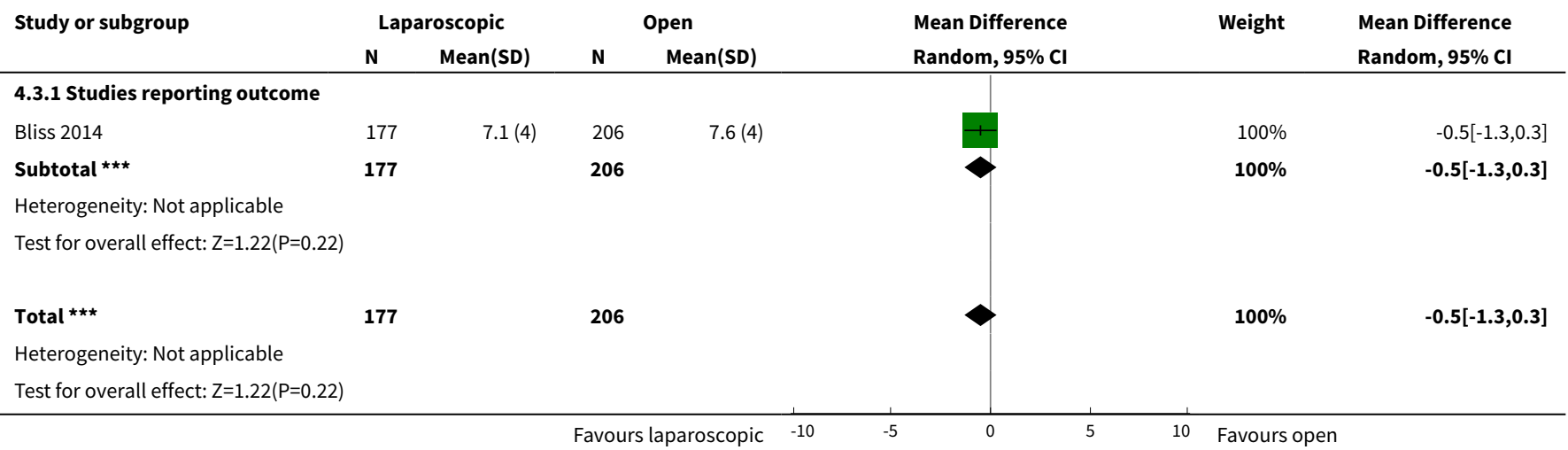


Analysis 4.4. Comparison 4 Subgroup analysis (publication date) - Laparoscopic versus open appendectomy in children, Outcome 4 Length of hospital stay.

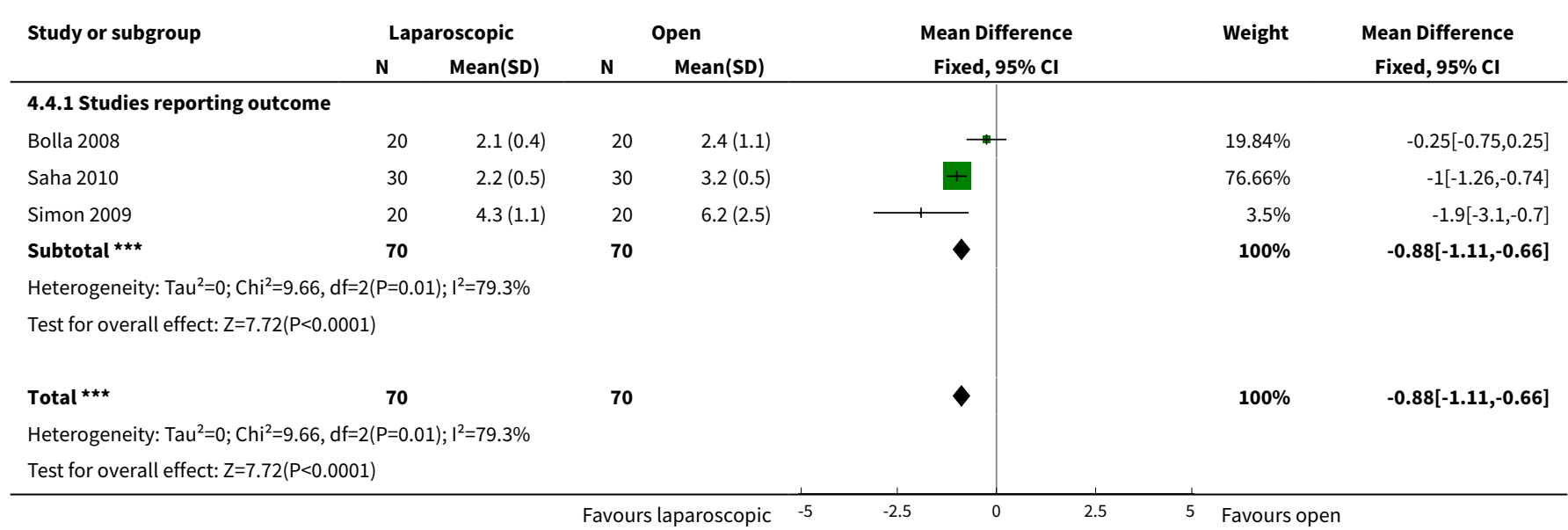

\section{APPENDICES}

\section{Appendix 1. CENTRAL search strategy}

\#1 MeSH descriptor: [appendix] explode all trees

\#2 MeSH descriptor: [appendicitis] explode all trees

\#3 MeSH descriptor: [appendectomy] explode all trees

\#4 append*:ti,ab.kw

\#5 (\#1 or \#2 or \#3 or \#4)

\#6 MeSH descriptor: [laparoscopy] explode all trees

\#7 (laparoscop* or laparotom*):ti,ab,kw

\#8 (\#6 or \#7)

$\# 9$ (\#5 and \#8)

\section{Appendix 2. MEDLINE search strategy}

1 exp appendix/

2 exp appendicitis/

3 exp appendectomy

4 append $^{*} . \mathrm{mp}$.

$5(1$ or 2 or 3 or 4$)$

6 exp laparoscopy/

7 (laparoscop* or laparotom).mp.

$8(6$ or 7$)$

9 (5 and 8$)$ 
10 randomized controlled trial.pt.

11 controlled clinical trial.pt.

12 randomized.ab.

13 placebo.ab.

14 clinical trial.sh.

15 randomly.ab.

16 trial.ti.

$17(10$ or 11 or 12 or 13 or 14 or 15 or 16$)$

18 exp animals/ not humans.sh.

$19(17$ not 18$)$

20 (9 and 19)

\section{Appendix 3. Embase search strategy}

1 exp appendix/

2 exp appendicitis/

3 exp appendectomy

4 append $^{\star} . \mathrm{mp}$.

$5(1$ or 2 or 3 or 4$)$

6 exp laparoscopy/

7 (open appendectomy or laparoscopic appendectomy).mp.

8 (laparoscop* or laparotom).mp.

$9(6$ or 7 or 8$)$

10 (5 and 9)

11 crossover procedure.sh.

12 double-blind procedure.sh.

13 single-blind procedure.sh.

14 (crossover ${ }^{\star}$ or cross over $\left.{ }^{\star}\right) . \mathrm{ti}, \mathrm{ab}$.

15 placebo*.ti,ab.11

16 (doubl $^{\star}$ adj blind $\left.{ }^{\star}\right) . t i$, ab.

17 allocat*.ti.ab.

18 trial.ti.

19 randomized controlled trial.sh.

20 random* $^{\star}$ ti,ab.

$21(11$ or 12 or 13 or 14 or 15 or 16 or 17 or 18 or 19 or 20$)$

22 (exp animal/ or exp invertebrate/ or animal.hw. or nonhuman/) not (exp human/ or human cell/ or (human or humans or man or men or wom?n).ti.) 
$23(21$ not 22$)$

24 (10 and 23)

Appendix 4. Criteria for judging risk of bias in the 'Risk of bias' assessment tool

\section{RANDOM SEQUENCE GENERATION}

Selection bias (biased allocation to interventions) due to inadequate generation of a randomised sequence

Criteria for a judgement of

'Low risk' of bias.

The investigators describe a random component in the sequence generation process such as:

- Referring to a random number table;

- Using a computer random number generator;

- Coin tossing;

- Shuffling cards or envelopes;

- Throwing dice;

- Drawing of lots;

- Minimization*.

*Minimization may be implemented without a random element, and this is considered to be equivalent to being random.

Criteria for the judgement of 'High risk' of bias.

The investigators describe a non-random component in the sequence generation process. Usually, the description would involve some systematic, non-random approach, for example:

- Sequence generated by odd or even date of birth;

- Sequence generated by some rule based on date (or day) of admission;

- Sequence generated by some rule based on hospital or clinic record number.

- Other non-random approaches happen much less frequently than the systematic approaches mentioned above and tend to be obvious. They usually involve judgement or some method of nonrandom categorization of participants, for example:

- Allocation by judgement of the clinician;

- Allocation by preference of the participant;

- Allocation based on the results of a laboratory test or a series of tests;

- Allocation by availability of the intervention.

Criteria for the judgement of 'Unclear risk' of bias.
Insufficient information about the sequence generation process to permit judgement of 'Low risk' or 'High risk'.

\section{ALLOCATION CONCEALMENT}

Selection bias (biased allocation to interventions) due to inadequate concealment of allocations prior to assignment

Criteria for a judgement of 'Low risk' of bias.
Participants and investigators enrolling participants could not foresee assignment because one of the following, or an equivalent method, was used to conceal allocation:

- Central allocation (including telephone, web-based and pharmacy-controlled randomisation);

- Sequentially numbered drug containers of identical appearance; 
- Sequentially numbered, opaque, sealed envelopes.

Criteria for the judgement of 'High risk' of bias.

Participants or investigators enrolling participants could possibly foresee assignments and thus introduce selection bias, such as allocation based on:

- Using an open random allocation schedule (e.g. a list of random numbers);

- Assignment envelopes were used without appropriate safeguards (e.g. if envelopes were unsealed or nonopaque or not sequentially numbered);

- Alternation or rotation;

- Date of birth;

- Case record number;

- Any other explicitly unconcealed procedure.

Criteria for the judgement of 'Unclear risk' of bias.
Insufficient information to permit judgement of 'Low risk' or 'High risk'. This is usually the case if the method of concealment is not described or not described in sufficient detail to allow a definite judgement - for example, if the use of assignment envelopes is described, but it remains unclear whether envelopes were sequentially numbered, opaque and sealed.

\section{BLINDING OF PARTICIPANTS AND PERSONNEL}

Performance bias due to knowledge of the allocated interventions by participants and personnel during the study

Criteria for a judgement of 'Low risk' of bias.
Any one of the following:

- No blinding or incomplete blinding, but the review authors judge that the outcome is not likely to be influenced by lack of blinding;

- Blinding of participants and key study personnel ensured, and unlikely that the blinding could have been broken.

Criteria for the judgement of Any one of the following:

'High risk' of bias.

- No blinding or incomplete blinding, and the outcome is likely to be influenced by lack of blinding;

- Blinding of key study participants and personnel attempted, but likely that the blinding could have been broken, and the outcome is likely to be influenced by lack of blinding.

Criteria for the judgement of Any one of the following:

'Unclear risk' of bias.

- Insufficient information to permit judgement of 'Low risk' or 'High risk';

- The study did not address this outcome.

\section{BLINDING OF OUTCOME ASSESSMENT}

Detection bias due to knowledge of the allocated interventions by outcome assessors

\section{Criteria for a judgement of} 'Low risk' of bias.

\section{Any one of the following:}

- No blinding of outcome assessment, but the review authors judge that the outcome measurement is not likely to be influenced by lack of blinding;

- Blinding of outcome assessment ensured, and unlikely that the blinding could have been broken.

\section{Criteria for the judgement of} 'High risk' of bias.

\section{Any one of the following:}

- No blinding of outcome assessment, and the outcome measurement is likely to be influenced by lack of blinding; 
- Blinding of outcome assessment, but likely that the blinding could have been broken, and the outcome measurement is likely to be influenced by lack of blinding.

Criteria for the judgement of 'Unclear risk' of bias.

\section{Any one of the following:}

- Insufficient information to permit judgement of 'Low risk' or 'High risk';

- The study did not address this outcome.

\section{INCOMPLETE OUTCOME DATA}

Attrition bias due to amount, nature or handling of incomplete outcome data

Criteria for a judgement of

'Low risk' of bias.
Any one of the following:

- No missing outcome data;

- Reasons for missing outcome data unlikely to be related to true outcome (for survival data, censoring unlikely to be introducing bias);

- Missing outcome data balanced in numbers across intervention groups, with similar reasons for missing data across groups;

- For dichotomous outcome data, the proportion of missing outcomes compared with observed event risk not enough to have a clinically relevant impact on the intervention effect estimate;

- For continuous outcome data, plausible effect size (difference in means or standardised difference in means) among missing outcomes not enough to have a clinically relevant impact on observed effect size;

- Missing data have been imputed using appropriate methods.
Criteria for the judgement of 'High risk' of bias.

\section{Any one of the following:}

- Reason for missing outcome data likely to be related to true outcome, with either imbalance in numbers or reasons for missing data across intervention groups;

- For dichotomous outcome data, the proportion of missing outcomes compared with observed event risk enough to induce clinically relevant bias in intervention effect estimate;

- For continuous outcome data, plausible effect size (difference in means or standardised difference in means) among missing outcomes enough to induce clinically relevant bias in observed effect size;

- 'As-treated' analysis done with substantial departure of the intervention received from that assigned at randomisation;

- Potentially inappropriate application of simple imputation.
Criteria for the judgement of 'Unclear risk' of bias.

\section{Any one of the following:}

- Insufficient reporting of attrition/exclusions to permit judgement of 'Low risk' or 'High risk' (e.g. number randomised not stated, no reasons for missing data provided);

- The study did not address this outcome.

\section{SELECTIVE REPORTING}

Reporting bias due to selective outcome reporting

Criteria for a judgement of

'Low risk' of bias.

\section{Any of the following:}

- The study protocol is available and all of the study's prespecified (primary and secondary) outcomes that are of interest in the review have been reported in the prespecified way; 
(Continued)

- The study protocol is not available but it is clear that the published reports include all expected outcomes, including those that were prespecified (convincing text of this nature may be uncommon).

Criteria for the judgement of

'High risk' of bias.
Any one of the following:

- Not all of the study's prespecified primary outcomes have been reported;

- One or more primary outcomes is reported using measurements, analysis methods or subsets of the data (e.g. subscales) that were not prespecified;

- One or more reported primary outcomes were not prespecified (unless clear justification for their reporting is provided, such as an unexpected adverse effect);

- One or more outcomes of interest in the review are reported incompletely so that they cannot be entered in a meta-analysis;

- The study report fails to include results for a key outcome that would be expected to have been reported for such a study.

Criteria for the judgement of

'Unclear risk' of bias.

Insufficient information to permit judgement of 'Low risk' or 'High risk'. It is likely that the majority of studies will fall into this category.

\section{OTHER BIAS}

Evidence of learning curve bias that might influence the internal validity of trial results

Criteria for a judgement of The study appeared to be free of learning curve bias.

'Low risk' of bias.

Criteria for the judgement of There is a potential source of bias. For example:

'High risk' of bias.

- The surgeons were not experienced in the surgical approaches;

- The authors identified a learning curve bias in their study.

Criteria for the judgement of

There may be a risk of bias, but:

'Unclear risk' of bias.

- Insufficient information to assess whether a learning curve bias exists.

WHAT'S NEW

\begin{tabular}{lll}
\hline Date & Event & Description \\
\hline 9 February 2018 & New search has been performed & This is an update of the review from 2010 \\
\hline 9 February 2018 & $\begin{array}{l}\text { New citation required but conclusions } \\
\text { have not changed }\end{array}$ & $\begin{array}{l}\text { Literature searches updated; } 18 \text { new trials added; risk of bias as- } \\
\text { sessment updated; summary of findings tables added; flow chart } \\
\text { diagram added; two new authors added in the byline. }\end{array}$ \\
\hline
\end{tabular}

\section{H I S T O R Y}

Protocol first published: Issue 3, 1999

Review first published: Issue 1, 2002 


\begin{tabular}{lll}
\hline Date & Event & Description \\
\hline 26 August 2010 & New search has been performed & review from 2004 updated \\
\hline 26 August 2010 & $\begin{array}{l}\text { New citation required and conclusions } \\
\text { have changed }\end{array}$ & $\begin{array}{l}\text { Literature searches updated, 13 new trials added, risk of bias as- } \\
\text { sessment updated, analyses and conclusion slightly changed, } \\
\text { author exchanged. }\end{array}$ \\
\hline 5 August 2008 & Amended & Converted to new review format. \\
\hline 22 July 2004 & $\begin{array}{l}\text { New citation required and conclusions } \\
\text { have changed }\end{array}$ & Substantive amendment \\
\hline
\end{tabular}

\section{CONTRIBUTIONS OF AUTHORS}

Mr. Jaschinski (TJ) screened articles against inclusion criteria, assessed the quality of the trials, extracted the data, and conducted most of the analyses.

Mr. Mosch (CM) assessed the quality of the trials and cross-checked the data extraction.

Dr. Eikermann (MEI) screened the articles against inclusion criteria and conducted the searches for ongoing and unpublished studies.

Prof. Neugebauer (NGB) helped in developing the idea for the review and contacted many of the trialists.

Dr. Sauerland (StS) assessed the quality of the trials, extracted the data from the literature, and wrote the first draft of the review.

All authors participated in writing the final draft of the review.

\section{DECLARATIONSOF INTEREST}

None.

\section{DIFFERENCES BETWEEN PROTOCOL AND REVIEW}

\section{Subgroup analysis}

We performed additional subgroup analyses for studies published within the last decade (as of 2007) in order to consider the changes in laparoscopy and imaging.

\section{INDEX TERMS}

\section{Medical Subject Headings (MeSH)}

*Laparoscopy [adverse effects]; Abdominal Abscess [epidemiology]; Acute Disease; Appendectomy [adverse effects] [methods]; Appendicitis [diagnosis] [ ${ }^{*}$ surgery]; Pain, Postoperative [epidemiology]; Randomized Controlled Trials as Topic; Recovery of Function; Surgical Wound Infection [epidemiology]; Time Factors

\section{MeSH check words}

Adult; Child; Female; Humans; Male 